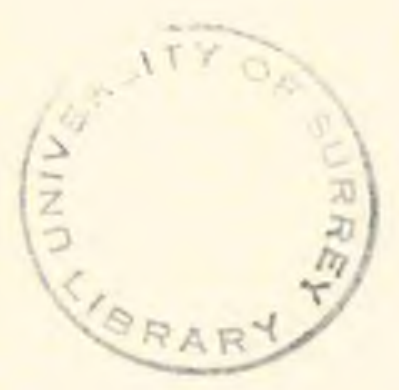

$1130528 \quad(5903052)$

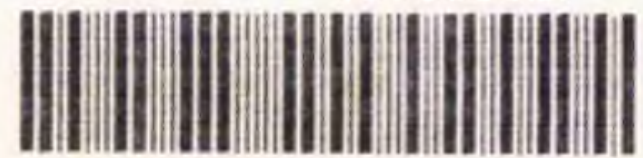

HIEHDLL, EM FFF'LICATI 


\section{All rights reserved}

\section{INFORMATION TO ALL USERS}

The quality of this reproduction is dependent upon the quality of the copy submitted.

In the unlikely event that the author did not send a complete manuscript and there are missing pages, these will be noted. Also, if material had to be removed, a note will indicate the deletion.

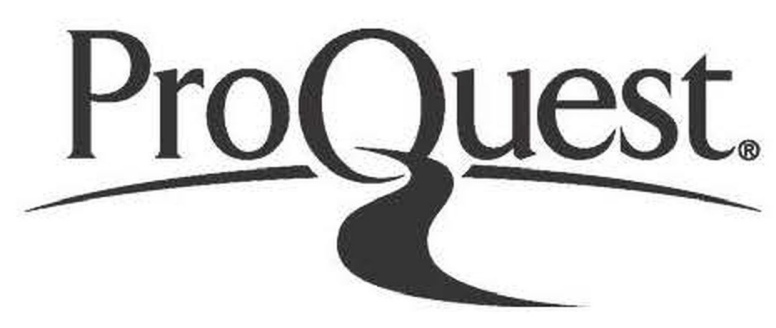

Published by ProQuest LLC (2017). Copyright of the Dissertation is held by the Author.

All rights reserved.

This work is protected against unauthorized copying under Title 17, United States Code Microform Edition (c) ProQuest LLC.

ProQuest LLC.

789 East Eisenhower Parkway

P.O. Box 1346

Ann Arbor, Ml 48106 - 1346 


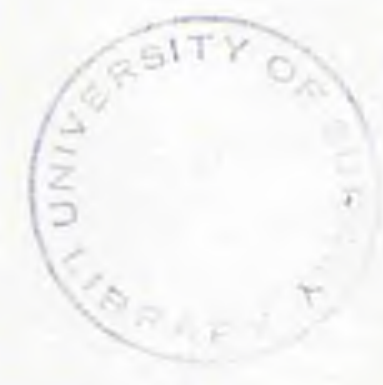


WAVEFRONT SHEARING OPTICAL INTERFEROMETER TO DIFFUSION MEASUREMENTS.

Thesis submitted for the degree of Doctor of Philosophy in the Faculty of Engineering of the University of London.

EDWARD M ${ }^{\mathrm{C}}$ KILLOP NICHOLL, Chemical Engineering Department, Battersea College of Technology, London. JULY 1966. 
0 


\section{ABSTRACT.}

A wavefront shearing optical interferometer has been built in this llaboratory, and its use for diffusion measurements is described.

In Part I of this work, the application of the interferometer to diffusion in liquid-1iquid systems is discussed. Using, a flowing-junction cell in conjunction with the interferometer, the following systems were Invesitigated and diffusion coefficients reported over a rarge of concentrations,

sucrose-watex; monoothanolaminewater; diethanolamine $\rightarrow$ water; and triethanolamine-water.

A new method for calculating the diffusivities of Iiquid-liquid systems has been proposed, and is used to obtain the diffusion coefficients for the above systems. A Ferranti sirius computer is used to handle the calculations.

The diffusion coefficients obtained in this Investigation were examined in comparison with existing diffusional theories and semi-empirical equations. It is. shown that these theories are inadequate, and it is also shown that the large deviations between the predicted diffusivities of the ethanolamines and the observed values are due, a) in part to the inadequacy of the equations, 
and b) in part to molecular irregularities of the ethanolamines in solution.

By explaining the molecular irregularitites of the ethanolamines in solution on the basis of a hydration effect, and calculating an approximate hydration number, a better agreement between the predicted and observed diffusivities is obtained.

In Part II of this workg the application of the wavefront shearing interferometer to gas-Iiquid diffusion Is described. The gas cell built in this luaboratory and the experimental technique is also described. Results for the systems $\mathrm{CO}_{2}-$ water, and $\mathrm{CO}_{2}$ into dilute monoethanolamine are reported. In the latter system the presence of a reaction zone is experimentally illustrated, and calculations are made explaining the movement of this reaction zone.

It is believed that this is the first time ever that a) the presence of this reaction zone has been shown experimentalily, and $b$ ) that the movement of the zone has been plotted.

At the moment, the application of this interferometer to gasmliquid diffusion is novel and needs improvement. When a fully successful experimental technique has been evolved, the possibilities for further woxk in this field are considerable. 
ACKDTOWI,EDGEMENTS.

The author would like to express his sincere gratitude to Dr.W.J. Thomas, Reader in the Department of Chemical Engtheering, Battersiea Colilege of pechnology, under whose. supervision this work was carried out. for his constant assistance and encouragement. The author is, indeed, indebted to Dr. Thomas for his advice concerning this workg and his supervision is deeply appreciated. The author also wishes to thanls the Science Research CounciI for the award of a Research studentship, covering toitition fees and living expenses for three years. The use of the recording microdensitometer, belonging to the Crystallography Department, Battersea College of Technology, is gratefully acknowledged.

Thanks are also due to Mr. W.Sliedzlewskig who took the photographs which 1Ilustrate this work.

The general assistance of the Laboratory staff, in particular Mr.C.R.Chesterman is also gratefuliy acknowledged. 


\section{CONTENTS.}

ABSTRACT。

ACKNOWLEDGEMENT.

IIST OF FTGURES.

vitii

LIST OF TABLES.

$x i$

NOTATION.

xiiit.

\section{PART I}

(IIquid-Iiquid Diffusion)

INTRODUCT ION.

Ch. I. THEORY OF DIFFUSION IN LIQUID SYSTEMS

I(a) The Diffusion Coefficient

I(b) Stokes-Einstein Hydrodynamic Theory

I(c) Kinetic Theories of Diffusion

I(d) Diffusion of Electrolytes.

$I(\theta)$ The Influence of Concentration on the Diffusion Coefficient

I(f) Semi-Empirical Equations for Predicting the Diffusion Coefficient.

Ch. II. THE EXPERTMENTAL DEIERMINATION OF DIFFUS ION COEFFICIENTS 
II(a) Steady State Methods 440

II(b) Restricted Diffusion 42

II(c) Free Diffusion 43

II(d) Optical Techniques for the Study of Diffusional Mass Transfer

Ch. III. THE WAVEFRONT SHEARING INTERFEROMETER 57 III(a) Theory

III(b) CaIculation of $D$ and $A t$

III(c) New Method for Calculating D 70

III(d) Apparatus $\quad 77$

III(e) Experimental Technique 83

Ch. IV. EXPERTMENTAL RESULTS 89

IV(a) Example of Determination of Diffusivity 89

IV(b) Diffusjonal Measurements for the Systems Sucrose-water, ethanolamines-water 96

IV(a) Tabulated Results 100

IV(d) Comparison of Results Obtained using W/s interferometer and those obtained by the Gouy method

Ch. $V$ THE APPLICAMTON OF THE SEMI-EMPIRICAL EQUATIONS TO ETHANOLAMINE-WATER DIFFUSION 135 
Ch.VI. THE PHYSICAI CHEMISTRY OF THE

ETHANOLAMINES IN SOLUTION.

1146

VI(a) Estimate of Ifydrate Formation from

Hygroscopicity Data

VI(b) Estimation of Hydration of Diethanolamine from Refractive Index Measurements

VI(c) Estimation of the Possible Hydration

of Ethanolamines from Vapour Pressuxe

Datea

Ch.VII. SUMMARY

VII(a) Suggestions for Further Work

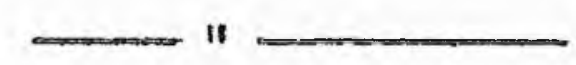

\section{PART II}

(Gas-Iiquid Diffusion)

INTRODUTTION

Ch. I. THEORY OF GASGIQUID DIFFUSION

1,82

I(a) Gas Absorption Without Reaction

185

I(b) Gas Absorption With Reaction

78.5

188

Ch. II. THE EXPERIMENTAL INVESTIGATION OF

GAS-IIQUID DIFEUSION。 
II(a) Sensitivity of the Wavefront Shearing Intiexferometer.

200

II(bi) Description of the Apparatus

205

II(c) Experimental Technique

Ch. III. EXPERTMENTAL RESULTS.

238

III(a) Phy'sical Absoretion of $\mathrm{CO}_{2}$ into water

218

III(b) Absorption of $\mathrm{CO}_{2}$ into dilute $\mathrm{MEA}$

225

Ch. IV. SUMMARY

APPENDIX Io Solution to Fick's and Iaw 262 APPENDIX II. Taylor Series Expansion of $\left(\frac{\Delta z}{\Delta x}\right)$

265

APPENDIX III. Computor Programme( Sirius Autocode) for Caliculation of $D$.

REFERENCES

274

PUBLISHED WORK ( Reprint )

280 


\section{IIST OF FTGURES.}

\section{PART I}

FignNo.

1. The Toeplex Scanning System

Page No.

2. Schlieren Gradient Recoraing

45

3. Mach Zender Interference Fringes

47

4. The Mach Zender Interferometer

47

5. The Gouy Interferometer

49.

6. The Rayleigh Interferometer

53

7. The Savart Plate

53

8. Interfexence Fringe Formation

58

60

9. The Wavefront Shearing Interferometer

76

10. The Flowing-Junction Cell (Photo)

80

11. Principle of the Flowing-Junction Cell

81

12. Photograms of Interference Fringes

85

13. Interference Fringes ( $0.0578 \%$ MEA ) (Photo)

86

14. Graphs of $\mathrm{s}$ and $(2 \mathrm{x})_{\mathrm{iB}}^{2}$ against $\Delta \mathrm{t}$

93

15. Graph of $(2 x)^{2}$ against $t(\Delta t=0)$

94

16. Graph of $(2 x)^{2}$ against $t \quad(\Delta t=0)$

95

17. Graph of Diffusivity against Concentration for MEA, DEA, and TEA

18. Graph of Diffusivity against Concentration

for MEA ( this work and Furzer ) 
19. Graph of Diffusivity against Concentration for DEA ( this work and Furzere )

20. Graph of Diffusivity against Concentration for TEA ( this work and Furzer)

21. Vapoux Pressures of the Ethanolamines 148

22. Viscosities of Ethanolamine Solutions 150

23. $D_{2} \mu$ against Concentration for the

ethanolamines.

152

24. Possible Amine Hydration against. Temperature 169

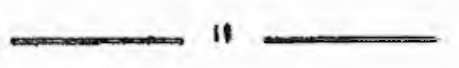

\section{PART II}

1. Whitman Film Theory Model 186

2. Higbie Penetration Theory Model 186

3. Hatta Film Theory Model 189

4. Curves of $\phi$ Vs. $\sqrt{M}$

189

5. Penetration Theory Model for Absorption

+ fast and order reaction 194.

6. Modification to the $\mathrm{w} / \mathrm{s}$ interferometer 204

7. The Gan-Liquid Cell 207

8. The Gas-Iiquid Cell (photo) 208

9. Arrangement of the Cell on the Bench 209 
10 Experimental Arrangements of the Gas Cell

IOA on the Optical Biench.

11. General Viow of the Optical Bench and

Gas, Gell (photo )

12. Camera, Timerecorder, and Viewer

13. Photograph of $\mathrm{CO}_{2}$ - water refractive index gradient Recording

14. Theoretical Concentration Profiles for $\mathrm{CO}_{2}$ water Diffusion

15. Diagrammatic Detail of Fig. 16

16. Photograph of the Concentration Profizes observed in the Absorption of $\mathrm{CO}_{2} \rightarrow \mathrm{MEA}$,

17. Series of Photos showing Movement of the Reaction Zone, in $\mathrm{CO}_{2}^{*} \approx$ MEA Absorption

18. Photograph of Turbulence in the Liquid Film 236

19. Photograph of Turbulence in the Bulk of the Iiquid

20. Graphs of $x^{\prime}$ and $x^{\prime \prime}$ against $\theta$ (run $I$ )

239

21. Graphs of $x$ against $\sqrt{\theta}$ 240

22. Solution to Penetration Theory Equation

246

23. Experimental and Theoretical $x^{1}, \theta$ curves

247

24. Possible $\mathrm{Cl}_{2} /$ Ferrous Chloride Absorption

KodeI.

25. Possible $\mathrm{CO}_{2}$ mEA Absorption Model 
LIST OF TABLES.

\section{PARTI I}

Table No.

Page No:

1. Fringe-Pair Movement, $D E A / 8$ :

91.

2.

Calculation of $\mathrm{D}, \quad \mathrm{DEA} / 8$

92

3.

Diffusion Coeffs. for Sucrose, MEA,

$\mathrm{DEA}$, and TEA-water.

99

4. Calculation of $\Delta$ t for Gouy Results.

129

5.

Summary of the Semi-Empirical Equations

142

Diffusivity Calculations.

6.

Properties of Pure Ithanolamines

$7: 47 ?$

7.

Viscosities of Ethanolamine Solutions

I5I

8.

The Product $D_{0} \mu$ for the Ethanolamines:

9.

Refractive Index Measurements for

DEA solutions

10. Calculation of DEA Hydration from.

Refractivity Data

2.64

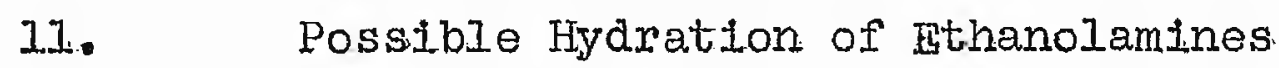

from Vapour Pressure Data

170

12. Estimated Hydration Numbers and Related

Molecular Volumes

171

13. Predicted Diffusivities Using Estimated

Hydration Numbers to recalculate $V_{A}$ 


\section{PART IT}

I. Experimental Diffusion of $\mathrm{CO}_{2}$ into water,

* recorded concentration gradients 222

2. Absorption of $\mathrm{CO}_{2}$ into $0.0166 \mathrm{~N} \mathrm{MEA}$,

- movement of the Reaction Zone 23.8

3. Calculation of $\sqrt{\alpha}$ from Least Squares fit to $\left(x_{a}^{2}-x_{b}^{2}\right),\left(\sqrt{\theta} a-\sqrt{\theta}{ }_{b}\right) \quad 242$ 
NOTATION.

Note: Numbers in parenthesis refer to a particular use of the symbol . 1.e. A, Axnold"s equation const. (36), refers to A's use in equation 36 only.

Where no number follows the description, the use is general.

\section{PART I}

A ; area. $\mathrm{cm}^{2}$

; Arnold's equation constant(36)

Bi : Arnold's equation constant 36)

b ; displacement between wavefronts

; Van der Vaals volume(57)

C ; Concentration

$\mathrm{g} / \mathrm{cm}^{3}$

D ; Mutual Diffusion Coefficient $\mathrm{cm}^{2} / \mathrm{sec}$

$D^{*} ;$ Self Diffusion Coefficient. $\mathrm{cm}^{2} / \mathrm{sec}$

$E_{\mu}, E_{\mathbb{D}} ;$ Activation energies for viscosity \& diffn, cals/g.mol $\Delta E_{\text {vap; }}$ energy of vaporisation

Fi Force opposing motion of particle (18)

; Semi-empirical facto Arnold's equation(36)

$G$; Magnification factor

$\triangle \mathrm{FH}_{\mathrm{vap}}$; Heat of vaporisation cals/g. mol

$J \quad ;$ Diffusional $f l u x \quad \quad g_{\circ} \operatorname{mol} / s_{s e c} \mathrm{~cm}^{2}$

K ; Boltzmann's constant (18)

External Ionic force (44) 
I ; Iatent heat of evaporation

cals: $/ \mathrm{g} \cdot \mathrm{mol}$

M ; Moleculars weight

Totali mass flow (12)

g.a $\mathrm{mols} / \mathrm{cm}^{2}$

Ni: $;$ Avogadros number $\left(=6.023 \times 10^{23}\right)$

Total number of observations (93)

n ; Refractive index

; Number of ions (43)

P ; Vapour pressure

mm. Flg:

R ; Gas constant

; Radius of particle (19)

$x$; Intermolecular spacing(39)

${ }^{\mathrm{G}_{\mathrm{H}}}$; Refractivity (Gladstone) (9.5)

$x_{I}$; Refractivity (Lorenz-Iorentz) (96)

$\mathrm{S}$; Area factor $\left(=\mathrm{V}_{\mathrm{A}}^{1 / 3}+\mathrm{V}_{\mathrm{B}}^{\mathrm{L} / 3}\right)$

$\mathrm{cm}$

s ; Standard error of estimate of $(2 \mathrm{x})^{2}$ on $t$

T ; Absolute temperature

$\mathrm{O}_{\mathrm{K}}$

t ; time

secs

$t_{i} ;$ time of inflexion of Gaussian curve

$\Delta t ;$ Zero-time correction factor

$\mathrm{U}$; Absolute velocity of cation (51)

$u$; Gation valency (5I)

velocity of particle (18)

$V$; Absolute velocity of anion (51)

; Molecular rolume at normal boiling point $\mathrm{cm}^{3}$ 


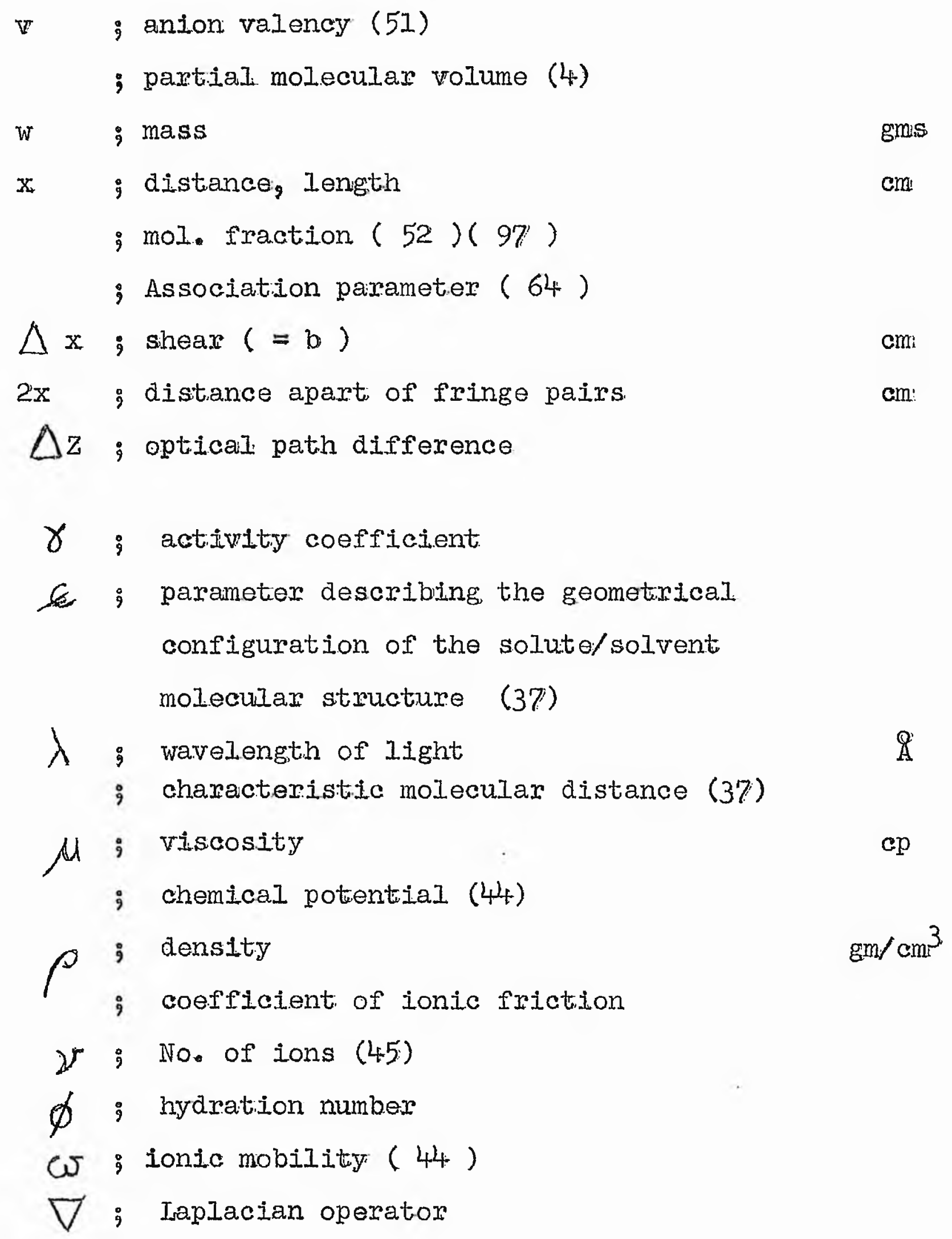


a ; optical path length thro cell $\mathrm{cm}$

c. ; concentration

$c_{\text {Ai }}$; interfacial concentration of gas in liquid

$\mathrm{c}_{\mathrm{BO}}$; initial concentration of solute in liquid

D ; gasmliquid diffusion coefficient

Hi ; Henry's law constant

$\mathrm{K}_{\mathrm{L}}^{\mathrm{O}}$; Iiquid film coefficient (physical absorp.) cm/sec

$\mathrm{K}_{\mathrm{I}}$; Liquid film coefficient (with reaction)

$K_{G} \quad$ g gas film coefficient

$x$; reaction velocity constant

$\mathrm{M} ;$ dimensionless group, $\mathrm{KD}_{\mathrm{A}} \mathrm{c}_{\mathrm{Bo}} /\left(\mathrm{K}_{\mathrm{L}}^{\circ}\right)^{2}$

$N$; rate of mass transfer

$n$; refractive index

p ; partial pressuxe

$q ;=c_{B O} / c_{A i}$

$\therefore \quad=D_{B} / D_{A}$

t ; time

$\mathrm{x}$; length, distance (general. ) cm

$\mathrm{x}_{\mathrm{L}}$; thickness of Iiquid film $\mathrm{cm}$

$x^{*}$; distance from interface to reaction zone cm

$x^{\prime \prime}$; distance from reaction zone to edge of 


\section{-xviiin}

$\alpha$; integration constant (equation 27)

$\theta ;$ time

$\lambda$; wavelength of light.

$\sigma ;=\left(\frac{\alpha}{D}\right)^{\frac{1}{2}}$

$\oint_{i}=K_{I_{1}}{ }_{L}$, ratio of mass transfer with and without reaction. 
$-1=$

PART I

The Application of the Wavefront Shearing Interferometer

to.

LiquiduIquid Diffusion. 


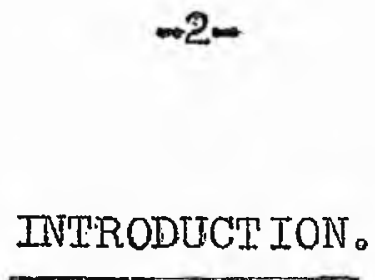

In many branches of chemical engineering a knowledge of diffusivity is of importance where liquid diffusion is rate-controlling in the mass transfex process. Examples include absorption, adsorption, liquidmliquid extraction, and dialysis. On the pure science side, the diffusion process is one of the aspects of Iiquid behaviour which must be described by atisfactory liquid-state theory, and hence diffusion rates are of importance in formulating and testing such theories. At the present moment, there is a distinct lack of precise diffusional theory, since the accurate measurement of diffusion rates is; only a comparatively recent achievement.

Diffusion studies were first made in this Iaboratory by Fuxzer ( 1) and Thomas ( 2 )。 The Gouy interferometer, an accurate optical technique, was used by the above workers to study the diffusion rates of the ethanolamines ( mono-gdi-g and tri-s) in water. The ethanolamines were chosen for investigation since they are widely used Industrially in gas absorption $\left(\mathrm{CO}_{2}\right.$ removal, natural gas sweetening ), and a knowledge of their diffusional properties is valuable in correlating mass 
transfer rates to absorption theory. Subsequent to Thomas and Furzer publishing their results,however, a criticism on their findings was made by Ibrahim and Kuloor ( 3 ). It was intimated that the disagreement between the experimental results and the theoretical predictions could be due to one of three causes, i.e.

1) Inaccurate work.

2) Inadequate diffusional theory.

3) Association or hydration of the ethanolamines in solution (though this cause was less favoured). It was therefore of some importance that a re-investigation be made of diffusion in the ethanolamine -water system, in order to examine Ibrahim and KuIoor's criticisms.

A highly accurate and sensitive interferometer was described by Bryngdahl ( 4 ), and it was realised that the capabilities of this, the wavefront shearing interferometer, surpassed those of any existing interferometer. It would thus be possible to make diffusion measurements of the greatest accuracy, and, as an added advantage, in very dilute solutions.

With this in mind, and the need to remexamine the diffusion rates in the ethanolamine-water systems, 
the wavefront shearing interferometer was constructed in this Iaboratory.

The scope of the first part of this work therefore takes the following form:

A review of the existing diffusion theories; a review of experimental techniques, and a brief survey of the sensitivities of these methods; a description of the theory, apparatus, and technique for the wavefront shearing interferometer; a report on the investigation, using the wavefront shearing interferometer, into the diffusion rates of the systems ethanolamine-water; a critical appraisal of the accuracy of the results obtained, and a comparison with the work of Thomas and Furzer; an assessment of the applicm ability of the diffusion theories to the experimental results; a study of the physical chemistry of the amines in order to attempt an explanation of the disagreement between the theoretical and observed diffusivities.

From this woxk it is hoped to make a recommendation for the future use of semi-empirical equations in predicting diffusivity. 


\section{$-5-$ \\ I. THEORY OF DIFFUSION IN LIQUID SYSTEMIS \\ Progress in the study of diffusion in liquid systems}

is only comparatively recent. Although the first measurements of diffusion were made in 1850, limitations due to inaccurate experimental equipment have kept the data in the literature down to an insufficiently small amount. Also, there is as yet no really clear understanding of diffusion in the liquid state, so that the available methods for estimating diffusion in Iiquid systems are not at all reliable.

Several theories of diffusion have been advanced, and using these theories semi-empirical correlations have been proposed. Many reviews have been made of both the existing theories and the experimental methods for the determination of the diffusion coefficient: Of particular note are those by Johnson and Babb (5), Nienow(6), Tyrell (7), Lightfoot and Cussier (8), Kamal and Canjar (9).

The purpose of this section is to give a background to the existing theories of diffusion and the available experimental methods. Having illustrated the present state of knowledge on the matter, it is proposed to show the importance of diffusion in mass-transfer operations (e.g. absorption, liquid-liquid extraction and dialysis). The diffusion coefficient will first be defined as a function of the concentration gradient in a diffusing system, and then the theories will be presented to show 


\section{$-6-$}

how the diffusion coefficient may be predicted as a function of the various physical properties of liquid systems.

I(a). The Diffusion Coefficient.

For Uni-directional diffusion, Fick (1855) established the simple relationship

$$
J=-D \frac{\partial \mathrm{c}}{\partial \mathrm{x}}
$$

now known as Fick's First Law. In words, this states that the rate of mass transfer is proportional to the concentration gradient at constant temperature and pressure. The minus sign indicates that the flow is opposite in sense to the direction of the concentration gradient.

In a system consisting of components $A$ and $B$, the diffusional flow of one component due to the concentration gradient must be balanced by that of the other. Writing the diffusion equation for each gubstance

$$
\begin{aligned}
& J_{A}=-D_{A} \frac{\partial C_{A}}{\partial x} \\
& J_{B}=D_{B} \frac{\partial C_{B}}{\partial x}
\end{aligned}
$$

The volume transfer will be equal to

$$
D_{A} v_{A}\left(\frac{\partial C_{A}}{\partial x}\right) \text { and } D_{B} v_{B}\left(\frac{\partial C_{B}}{\partial x}\right)
$$

where $v_{A}$ and $v_{B}$ are partial molar volumes

For a constart volume system

$$
D_{A} v_{A}\left(\frac{\partial C_{A}}{\partial x}\right)+D_{B} v_{B}\left(\frac{\partial C_{B}}{\partial x}\right)=0 \quad \cdots . . .(4)
$$

The quantity of $A$ per unit volume overall is $v_{A} C_{A}$ and that of $B$ is $v_{B} C_{B}$ 


$$
\begin{aligned}
& -7- \\
& \therefore \mathrm{v}_{A} \mathrm{C}_{\mathrm{A}}+\mathrm{v}_{\mathrm{B}} \mathrm{C}_{\mathrm{B}}=1 \\
& \mathrm{v}_{\mathrm{A}}\left(\frac{\partial \mathrm{C}_{A}}{\partial \mathrm{x}}\right)+\mathrm{v}_{\mathrm{B}}\left(\frac{\partial \mathrm{C}_{\mathrm{B}}}{\partial \mathrm{x}}\right)=0 \ldots . \cdot(6)
\end{aligned}
$$

For equations (4) and (5) to be true it follows that $D_{A} \equiv D_{B}$

Hence, in the standard binary systems to be considered, the diffusion coefficient measured will be the one common to both components, $D_{A B}$.

If the mass transfer is considered over an elemental volume $\delta v=A \cdot \delta x$, the diffusional flow at $x+\delta x$

$$
=J+\left(\frac{\partial J}{\partial x}\right) \cdot \delta x
$$

and

$$
\begin{aligned}
& \frac{\partial}{\partial t}(J)=-\frac{\partial}{\partial x}(J) \delta x \\
& \delta x \cdot\left(\frac{\partial C}{\partial t}\right)=\frac{\partial}{\partial x}\left(D \cdot \frac{\partial c}{\partial x}\right) \delta x \quad \cdots . . .(9) \\
& \frac{\partial \mathrm{C}}{\partial \mathrm{t}}=\mathrm{D} \cdot \frac{\partial^{2} \mathrm{C}}{\partial \mathrm{x}^{2}} \quad \cdots \cdot \cdot(10)
\end{aligned}
$$

This equation is known as Fick's Second Law.

The second Law is almost exclusively employed in the experimental deremination of $D_{A B}$ since it is far easier to measure concentrations than molecular flux,

Solutions of the equation $\frac{\partial \mathrm{C}}{\partial t}=\mathrm{D} \frac{\partial^{2} \mathrm{C}}{\partial \mathrm{x}^{2}}$ have been

developed by Fourier series analysis, and are necessary for an evaluation of $D_{A B} \cdot$ Crank (10) and Jost (11) present the solutions for different boundary conditions. 
$-8-$

Solution of Hick's Second Law for a uniform cross-section cylinder of infinite length (extended initial. distribution) :-

$$
\text { at } \mathrm{t}=\mathrm{O}, \quad \begin{array}{ll}
\mathrm{C}=\mathrm{C}, & \mathrm{x}<0 \\
& \mathrm{C}=0, \mathrm{x}>0
\end{array}
$$

By differentiating $\frac{\partial \mathrm{C}}{\partial t}=\mathrm{D} \cdot \frac{\partial^{2} \mathrm{C}}{\partial \mathrm{x}^{2}}$, a solution (for the above conditions) is obtained as

$$
c=\frac{A}{\sqrt{t}} \cdot \exp \left(-x^{2} / 4 D t\right) \quad \cdots \cdots(11)
$$

where $A$ is an arbitrary constant.

(Please see appendix)

If the total amount of material diffusing

$$
=\mathbb{M}=\int_{-\infty}^{\infty} \mathrm{C} \cdot \mathrm{dx}
$$

and writing $x^{2} / 4 D t=z^{2}, \quad d x=2 \sqrt{D t} \cdot d z$

then $M=2 A \sqrt{D} \int_{m \infty}^{30} \exp \left(-Z^{2}\right) d Z=2 A \sqrt{\pi D} \cdots \cdots$ (12)!

But $A=\sqrt[c]{t} / \exp \left(-\frac{x^{2}}{4 D t}\right)$

Substituting (13) into (12)

$$
C=\frac{M}{2 \sqrt{\pi D t}} \exp \left(-\frac{x^{2}}{4 D t}\right)
$$

Hence the concentration distribution at time $t$ is

$$
\frac{\partial c}{\partial x}=\frac{\Delta c}{2 / \pi D t} \exp \left(-\frac{x^{2}}{4 D t}\right)
$$

where $\Delta \mathrm{C}=\mathrm{C}_{\mathrm{O}}-\mathrm{C}$ 
If it is assumed that the refractive index is linearly related to the concentration, then

$$
\frac{\partial n}{\partial x}=\frac{\Delta n}{2 \sqrt{\pi D t}} \exp \left(-\frac{x^{2}}{4 D t}\right)
$$

This relationship, derived from a solution of Fick's

second law is used almost exclusively where optical techniques are used to measure the diffusion coefficient.

It will: be shown later how equation (16) is used to evaluate $D$ from a knowledge of the refractive index gradient of the diffusion system.

Crank (10) gives a particularly thorough study of the other possible solutions of Fick's second law. Solutions include:

$$
\begin{aligned}
& \text { Semi-infinite cylinder, } \\
& \text { finite cylinder, } \\
& \text { diffusion in a sphere, } \\
& \text { diffusion with a moving boundary etc. }
\end{aligned}
$$

If $D_{A B}$ is depenant on concentration, then there might be experimental errors in the determination of $D_{A B}$ if the concentration difference between the two solutions is significant. Strictly speaking, $D_{A B}$ as calculated by the Fick equation, is for a point condition only.

With this criterion in mind, two types of diffusion coefficient can be defined:- 
1) Differential Diffusion coefficients, in which diffusion occurs between two solutions of only differential concentration difference.

2) Integral diffusion coefficients, in which diffusion occurs between two solutions of significant concentration difference.

The integral coefficient is related to the differential coefficient, $\mathrm{D}_{\mathrm{AB}}$, by the expression

$$
\overline{D_{A B}}=\frac{I}{C_{1}-C_{2}} \int_{C_{2}}^{C_{1}} D_{0} \mathrm{dC}
$$

Since the differential coefficient is the one needed for any examination of the physical properties of the solution, then it is desirable to make the concentration difference as small as possible when measuring $D_{A B}$, - and the coefficient is thus taken as measured at the mean concentration.

Theories of Diffusion

In this section the principal theories of diffusion are discussed, in which the diffusion coefficient is related to the physical properties of the solution.

The theories may be categorised as below:-
a) Non-electrolvtes
b) Electrolytes

Hydrodynamic theories

Kinetic theories 
I(b) Stokes-Einstein Hydrodynamic Theory

Tyrell discusses the hydrodynamic theories in detail, but the essentials of what is known as the stolses-Einstein theory are adequately described by Gainer and Metzner (12) Tuwiner (13) and Glasstone (14).

Although the hydrodynamic theory has some value for the diffusion of large molecules (e.g. colloids), its use is not suggested at all generally. The derivation of the theory is due independently to Sutherland and Einstein. The Nernst-Einstein equation is intermediate, i.e.

$$
D_{A B}=k \cdot T \cdot \frac{u_{A}}{F_{A}}
$$

where $k=$ Boltzmanns constant

$$
\begin{aligned}
T & =\text { absolute temperature } \\
u_{A} & =\text { velocity of particle } \\
F_{A} & =\text { force opposing motion of particle } A
\end{aligned}
$$

The diffusion flow is regarded as a balance between a driving force and a resistance to flow which leads to a constant average diffusion velocity.

For a particle moving through a liquid the stokes drag is given by

$$
\mathrm{F}_{\mathrm{A}}=6 \pi \mu_{\mathrm{B}} \cdot \mathrm{u}_{\mathrm{A}} \cdot \mathrm{R}_{\mathrm{A}}
$$

where $\mu_{B}=$ viscosity of the solvent

$R_{A}=$ radius of the diffusing particle 


\section{-II2-}

On substitution into (18) the Stokes-Einstein equation is obtained

$$
D_{A B}=\frac{k \cdot T .}{6 \pi \mu_{B} \cdot R_{A}}
$$

$\mathrm{R}_{\mathrm{A}}$ may be computed from

$$
M_{A}=\frac{4}{3} \pi R_{A}^{3} \rho_{B} N
$$

where $\mathbb{M}_{A}=$ molecular weight

$$
\begin{aligned}
& \rho_{B}=\text { density } \\
& N \quad=\text { Avogadros number }
\end{aligned}
$$

Originally the driving force was considered to be the gradient of osmotic pressure, but nowadays it is taken as the gradient of chemical potential of the diffusing species. Thus an activity correction term should be included with equation (20).

i.e.

$$
D_{A B}=\frac{k_{0} T}{6 \pi A_{B} R_{A}}\left[1+\left(\frac{\partial \ln \gamma_{A}}{\partial \ln c_{A}}\right)_{T_{2} P}\right] \cdots
$$

where $\gamma=$ activity

$$
c=\text { molar concentration }
$$

For ideal solutions this activity term is unity.

For diffusion in water the Stokes-Einstein equation is only applicable where the solute molecular weight exceeds 300 , and as with all other theories to be discussed applies only to dilute solutions. 
I(c) Kinetic Theories of Diffusion

There are two main kinetic theories of diffusion, Arnold's and Eyring's.

\section{Arnold's Kinetic Theory (15)}

Arnold applied the kinetic theory of gases, which had been successful in interpreting the mutual diffusion coefficients of gaseous systems, to Iiquid systems. Many of the reviews of liquid diffusion only summarise Arnold's theory since its use is limited in applicability due to the presence of three empirical constants. However, to understand Arnold's approach to liquid diffusion the development of the theory will be given here.

As mentioned above the classical kinetic theory for gaseous diffusion is applied to liquid systems, making allowances for the difference in the packing of the molecules. From eqn。(1), Fick's First Law,

$$
J=\frac{d M}{d t}=D \cdot A \cdot \frac{d c}{d x}
$$

where $\mathbb{M}=$ total mass flow

If the net forward velocity of a molecule in the diffusion stream is represented by $u$, then

$$
\frac{\mathrm{dM}}{\mathrm{A} \cdot \mathrm{dt}}=\mathrm{C} \cdot \mathrm{u}
$$

It is assumed that the acceleration of the diffusing molecule is negligible.

The driving force $d c / d x$ is used solely to overcome the 


\section{$-14+\infty$}

resistance to diffusion, measured in terms of the momentum transferred from the diffusing molecule to the solvento This momentum transfer is equal to the product of the total number of collisions of unlike molecules by the momentum loss per collision.

From gas theory, the number of collisions per second Is proportional to

1) Concentration of each type of molecule。

2) Average cross-sectional area.

3) Root mean square volocity.

$$
\begin{aligned}
& \text { i.e. } c_{1} \cdot c_{2} \cdot s^{2} \cdot \sqrt{u_{1}^{2}+u_{2}^{2}} \\
& \text { where } s=v_{1}^{1 / 3}+v_{2}^{1 / 3}
\end{aligned}
$$

$$
\text { ( } V=\text { molecular volume) }
$$

At constant temperature all molecules have the same kinetic energy. Since mu ${ }^{2}=$ constant, then (25) becomes

$$
c_{1} \cdot a_{2} \cdot s^{2} \sqrt{\frac{I}{\mathrm{M}_{1}}+\frac{1}{\mathrm{M}_{2}}}
$$

For forceless spheres, the momentum loss per collision is

$$
=2 / 3 \cdot \frac{M_{1} \cdot M_{2}}{M_{1}+M_{2}} \cdot\left(u_{1}+u_{2}\right)=2 / 3 \cdot \frac{\left(u_{1}+u_{2}\right)}{1 / M_{1}+1 / M_{2}}
$$

Assuming no volume change on mixing,

$$
c_{1} V_{1} u_{1} \Rightarrow c_{2} v_{2} u_{2}=0
$$

Since $c_{1} V_{1}+c_{2} V_{2}=1$ 


\section{$-1.5$}

Combining (29) and (30),

$$
\left(u_{1}+u_{2}\right)=u_{1 /} / c_{2} \circ v_{2}
$$

From (27), (28), and (3.I) the resistance to diffusion is proportional to

$$
\frac{c_{1} \cdot c_{2} \cdot s^{2} \cdot u_{1}}{c_{2} \cdot v_{2} \cdot \sqrt{I / M_{1}}+I / M_{2}}
$$<smiles>CC[18OH]</smiles>

From. (23) and (24), $\frac{d c_{1}}{d x}=\frac{c_{1} w_{1}}{D}$

Solving for $D_{9}$

$$
\mathrm{D}=\mathrm{B} \cdot \frac{\sqrt{I / M_{1}+I / M_{2}}}{\mathrm{~s}^{2}} \cdot V_{2}
$$

where $B$ Is a proportionality constant.

Equation (34) fails on three main assumptions used in its derivation, the assumptions being that.

i) all collisjons are binary; ii) the collision rate is unaffected by the molecular volumes; and $i$ i.i) that intermolecular forces are negligible.

These assumptions cannot be allowed in liquid diffusion. Arnold overcame these objections by bringing in the semi-empirical factor $F$ into equation (34)。 For dilute systems, $F$ is essentially a function of the solvent properties only, and is proportional to the square 
root of the solvent viscosity.

When either solvent or solute or both are associated, association factors $A_{1}$ and $A_{2}$ are included in the expression for $\mathrm{F}$.

Writing $F=A_{1} A_{2} V_{2} \sqrt{\mu_{2}}$

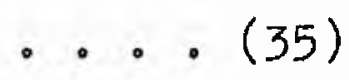

we get $D=B \cdot \sqrt{\frac{\frac{1}{M_{1}}+\frac{1}{M_{2}}}{A_{1} A_{2} S^{2} \sqrt{\mu_{2}}}}$

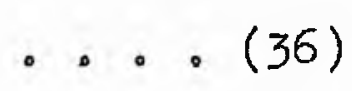

For water, $A_{2}=4.7$ and $B=0.0108$ at $25^{\circ} \mathrm{C}$

Eyring's Theory

Eyring and co-workers applied absolute rate theory to diffusion, and this was presented in a number of papers. A review of all this work is given by Glasstone et al, (16) and Kincaid et al (17). The theory is also summarised in other references, notably by Tyrell (7), Johnson and Babb (5), and Gainer and Metzner (12).

The Eyring theory attempts to explain the diffusion process on the basis of a cell model for the liquid, in which molecules move from a given position to an adjacent hole in the Iiquid structure. Eyring assumed that the energy of activation for the diffusion process is that energy required to form the extra space in the liquid to allow the molecules to move. A similar argument was applied to viscous flow. 
An equation in which the diffusion coefficient is considered as a function of both the activation energy for diffusion and the activation energy for viscous flow was arrived at, i.e.

$$
D=\frac{K \cdot T}{\epsilon \cdot \mu_{B}} \cdot\left(\frac{\lambda_{a}}{\lambda_{b} \lambda_{c}}\right) \frac{\lambda_{1}^{2}}{\lambda_{2}^{2}} \cdot \exp \left(\frac{E_{\mu_{B}}-E_{D(A B)}}{R T \cdot}\right)
$$

where $K=$ Boltzmann's constant

$$
\begin{aligned}
& E_{\mu}=\operatorname{acti} \text { ation energy for viscosity } \\
& E_{D}=\quad " \quad \text { " }
\end{aligned}
$$

$\lambda_{1}^{2} / \lambda_{2}^{2}=$ ratio of partition functions for molecules in the equilibrium and activated states.

$E=$ parameter describing the geometrical configuration of the solute/solvent molecular structure

$\lambda_{a}, \lambda_{b}, \lambda_{c}=$ characteristic distances as below.
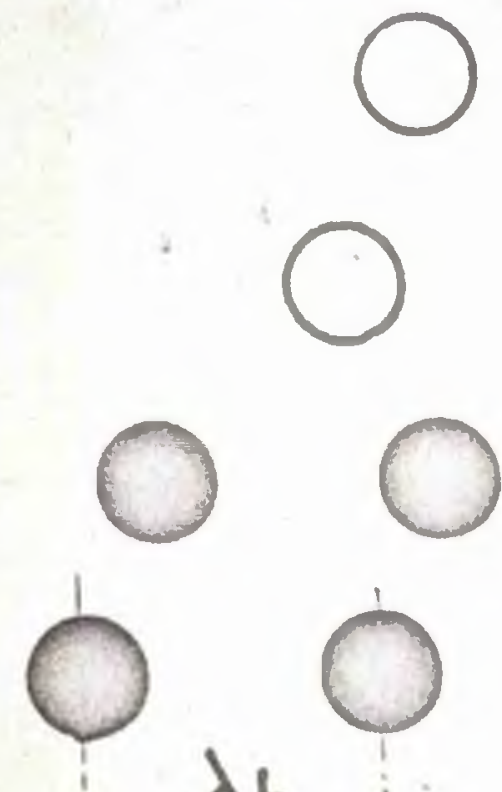

$\lambda_{b}$
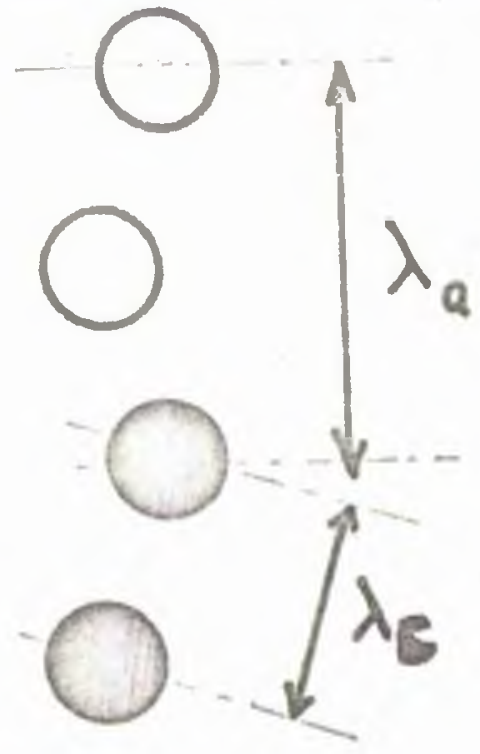

Migrating entities,

$\lambda$ represented by white circles, are moving in viscous flow under the influence of shearing forces. 


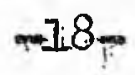

The equation may be simplified by putting

$$
\begin{aligned}
& \text { 1) } \left.\begin{array}{l}
\lambda_{1}^{2} / \lambda_{2}^{2} \\
\text { 2) }\left(\lambda_{a} / \lambda_{b} \lambda_{c}\right.
\end{array}\right)=\left(N / V_{B}\right)^{I / 3}
\end{aligned}
$$

Where $\mathrm{N}=$ Avogadros number

Thus we may write the equation for the diffusion coefficient

$D=\frac{K_{0} T_{0}}{E \mu_{B}}\left(\frac{N T}{\bar{V}_{B}}\right)^{I / 3} \exp \cdot\left(\frac{E \mu(B)-E E_{D}(A B)}{R_{0} T_{0}}\right) \cdot .(38)$

Although Eyring and co-workers assumed that $\mathrm{E}_{\mu}=\mathrm{E}_{\mathrm{D}}$, this is only an approximation that is applicable to self diffusion.

Gainer and Metzner (12) have made a useful analysis of the Eyring theory based on viscosity and diffusivity cage models. They have put forward an equation for the calculation of $E_{\mu(B)}-E_{D}(A B)$

i.e. $E_{\mu(B)}-E_{D(A B)}=\frac{E_{\mu(B)}}{2}-\left(\frac{E A}{E B}\right) \sqrt{\frac{r_{A A}}{r_{A B}} \cdot \frac{E^{\mu(A-H)}}{2} \cdot \frac{r_{B B}}{r_{A B}} \cdot \frac{E_{\mu(B-H)}}{2}}$

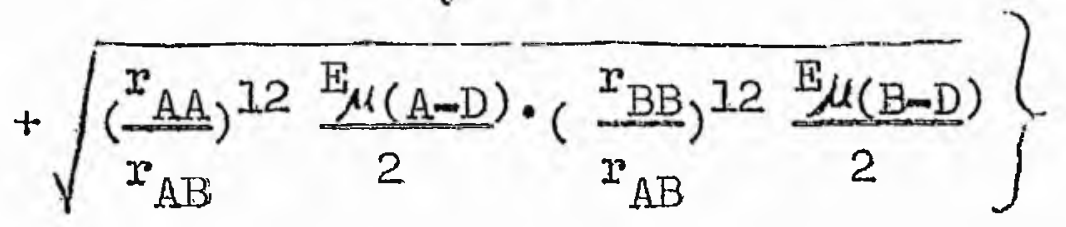

$r_{A A}, r_{B B}, r_{A B}$ are intermolecular spacings,

$\mathbb{I}_{\mathrm{AA}}=\left(\frac{V_{A}}{N}\right)^{I / 3}, \quad \mathbf{r}_{\mathrm{BB}}=\left(\frac{V_{B}}{N}\right)^{I / 3}, r_{A B}=\frac{r_{A A}+r_{B B}}{2}$ 
$E_{\mu(A, H),} E_{\mu(B-H)}$ are the activation energies due to hydrogen bonding.

$E_{\mu(A-D),} E_{\mu(B-D)}$ are the activation energies due to dispersion effects.

The above two energies are taken on the assumption that the total activation energy may be divided into two parts for a compound in general. They are calculated on the basic assumption that the total heat of vaporisation of a compound is formed of two parts, i.e.

$$
\begin{array}{lll}
\Delta \mathrm{H}_{\mathrm{vap}} & \Delta_{\mathrm{vap}}+\Delta \mathrm{H} & \operatorname{vap}(\mathrm{x}-\mathrm{H}) \\
\text { at } \mathrm{T}_{\mathrm{R}} & \text { of Hydrocarbon } & \text { due to } \\
& \text { homologue at } \mathrm{T}_{\mathrm{R}} & \text { hydrogen } \\
& & \text { bonding }
\end{array}
$$

$$
\therefore \frac{E_{\mu(x-H)}}{E_{\mu(x)}}=\frac{\Delta H_{\text {Vap }}(x-H)}{\Delta^{V_{\text {Vap }}(x)}}
$$

and.

$$
E_{\mu \cdot(x-D)}=E_{\mu(x)}-E_{\mu}(x-H)
$$

This calculation may be done quite easily for say, alcohols, which have a homologue, and Gainer and Metzner refer to the method. But the whole of the above calculation is somewhat invalidated when no homologue can be found (as with the amines)。

$\mathrm{E}_{\mu(\mathrm{x})}$ is calculated from

$$
E_{\mu}(\mathrm{x})=\operatorname{RP} \ln \left[\frac{\mu v^{2 / 3} \Delta \mathrm{E}_{\operatorname{vap}}}{\left(1.09 \times 10^{-3}\right) \mathrm{M}^{\frac{1}{2} \mathrm{~T}} 3 / 2}\right] \quad \cdots .(42)
$$




\section{$-20-$}

The parameters $E_{A}, \epsilon_{B}$ have been taken by Gainer and Metzner to be 6 for all the compounds that they considered, apart from ethyl and methyl alcohols to which they ascribed the value 8 .

In actual fact the variation of this parameter is quite considerable from compound to compound, so that it effectively becomes a semi-empirical constant which introduces a factor of inaccuracy.

\section{I(d) Diffusion of Electrolytes}

A comprehensive description of the theory of the diffusion of electrolytes is given by Harned and Owen (18), and Tyrel1 (7). Tuwiner (13) presents a fairly basic description that is of use for a general understanding of the principles involved. Obviously, the treatment of diffusion of electrolytes must differ fairly radically from that of non-electrolytes due to the formation of ion pairs in solution. As a requirement of electrical neutrality, the anions and the cations must move at an equal rate. Thus the mobility is intermediate between that of the component ions when these are free of electric forces.

\section{Theory of Diffusion of a Simple Electrolyte}

For an electrolyte solution containing two types of ions,

$u_{1}=u_{2}=u_{1}$

(ionic velocity)
$J_{1}=n_{1} u$

$J_{2}=n_{2} u$

(flow of ions) 
At low concentrations, interaction between the ions may be neglected, and

$$
u=K_{1} \omega \sigma_{1}=K_{2} \omega \tau_{2}=-\omega_{1} \nabla_{\mu_{1}}=\omega_{2} \nabla_{\mu_{2}} \ldots\left(4 \mu_{1}\right)
$$

$K=$ external forces on the ions

$\omega=$ mobility of the ions

$\nabla=$ Laplacian operator

$\mu=$ chemical potential

If $\mu$ is the chemical potential of a molecule which breaks up into $\nu_{1}$ anions and $\nu_{2}$ cations, then $\mathrm{K}=\nu_{1} \mathrm{~K}_{1}+\nu_{2} \mathrm{~K}_{2}=-\nabla \mu$

From (44) and (45)

$$
K_{2}=\left(\frac{\omega_{1}}{\omega_{2}}\right) K_{1}=\left(\frac{\rho_{2}}{\rho_{1}}\right) K_{1}
$$

where $\rho=$ coefficient of friction of the ions)

$$
K_{1}=-\frac{\rho_{1}}{\nu_{1} \rho_{1}+\nu_{2} \rho_{2}} \cdot \nabla \mu=-\frac{\omega_{2}}{\nu_{1} \omega_{2}+\nu_{2} \omega_{1}} \nabla_{\mu} \ldots \text { (47) }
$$

and $u=-\frac{\omega_{1} G J_{2}}{v_{1} \omega \sigma_{2}+v_{2} \omega_{1}} \cdot \nabla \mu$

If the solution is ideal

$$
\nabla_{\mu}=\left(\nu_{1}+\nu_{2}\right) k \frac{\nabla^{n}}{n}
$$

and consequently

$$
J=n V=-\frac{\left(\nu_{1}+\nu_{2}\right) \omega_{1} \omega_{2}}{v_{1} \omega_{2}+\nu_{2} \omega_{1}} \nabla_{n}
$$

This expression is known as Nernst's Law of diffusion. Written in a simplified form, Nexnst's Law may be expressed 
$D=8.314 \times 10^{-7} \cdot \frac{\mathrm{UV}}{\mathrm{U}+\mathrm{V}}\left\{\frac{1}{\mathrm{u}}+\frac{1}{\mathrm{~V}}\right\} \cdot \mathrm{T} \quad \ldots(51)$

where $U$ and $V$ are absolute velocities of cation and anion respectively under a force of 1 dyne at infinite dilution. $u$ and $v$ are the valencies of the cation and anion.

This equation is not applicable to weak electrolytes where solutions consist of a mixture of ions and large numbers of undissociated molecules.

Tables may be found in the Iiterature for $U$ and $V$, but are mostly given only for strongly ionised salts. Where values of $U$ and $V$ are given, the equation is most useful for engineering purposes.

However, since the electrolyte moves in one direction, and the solvent by replacing it moves in the opposite direction, an "electrophoretic" effect exists. This electrophoretic effect is allowed for in two expressions developed by Onsager and Fuoss (19).

Together with a thermodynamic term, - which allows for the activity coefficient of an electrolyte varying throughout the diffusing system, the two electrophoretic terms may be combined with the Nernst equation to give a comprehensive expression for the diffusion coefficient of an electrolyte in solution.

Harned and Owen give the development of this expression. It is not intended to give the development 
here since it is somewhat lengthy ${ }_{2}$, and the rinal results (although given hexe) are more: of use to a pure physital chemist;

The full equation is.

$$
\begin{aligned}
& D=\left(x_{1}+\partial_{2}\right) \cdot 1000 R \cdot T\left(1+c \cdot \frac{\partial \ln y_{5}}{\partial c}\right) \\
& x\left(1.0701 \times 10^{-30} \cdot \frac{\lambda_{1}^{0} \lambda_{2}^{0}}{y_{1} 10_{1}}+\frac{\Delta 0^{0}}{6}+\frac{\Delta 0^{\prime \prime}}{6}\right)
\end{aligned}
$$

where $A m^{\prime}$ and $\Delta M^{\prime \prime}$ are the electrophoretic enfect terms given: by

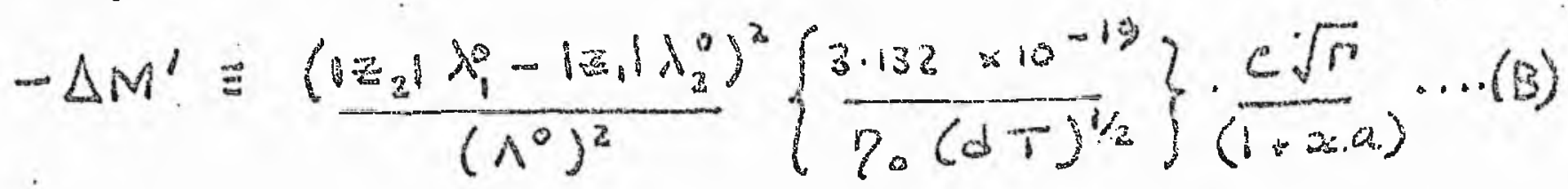

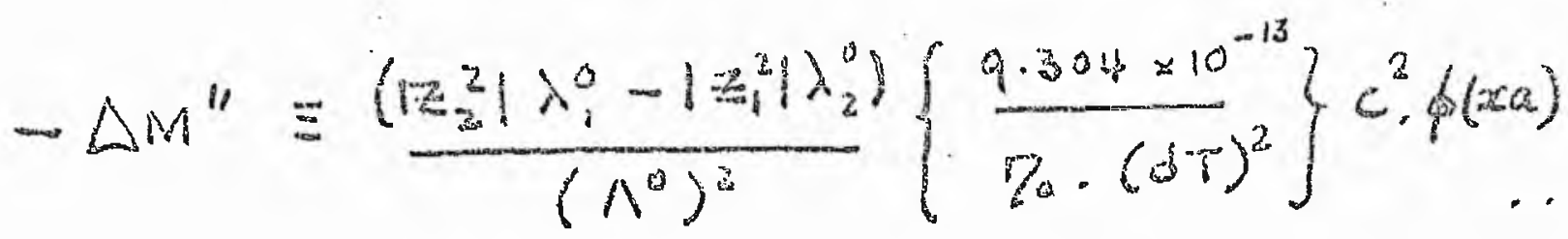

where $f^{\prime}(x a)=e^{2} \cdot E_{i}(2 x a) /(1+x a)^{2} \ldots(D)$

The teem $\left(1+6 \cdot \frac{\partial \ln y}{\partial c}\right)$ is the thermodynamic term which allows fos the vaviation of the activity coefficient throughout the diffusing system. The nomenciature for equations A to E (additional to nomenclature already used) is. givien below:- 
Nomenclatuxe fox equations $A$ to $E_{i-}$

$R=$ Gas constant

$I^{2}=$ Absolute tempexature

$c=$ molecular concentration

$y_{-}^{*}=$ mean molas activity coefficlent of the electrolyte

$\left.\lambda_{i}^{0}\right\}=$ Iimiting; equivalent conductance of the jons $\lambda_{2}^{0}$ indivated, at infinite dilution.

$\left.\begin{array}{c}z_{z_{1}} \\ z_{2}\end{array}\right\}=$ valencios.

$\Lambda_{0}=$ Iimiting equivalent conauctance of the electrolyte at infinite dilution.

$\eta_{0}=$ Viscosity of the solvent.

d. ajelectric: constant of the solution.

$\Gamma^{\prime}=$ jonal concentration.

$x=$ reciprocal of average radius of the ionic atmosphere.

$a_{2}=\operatorname{activit}{ }^{2}$

$\mathrm{E}_{i}()=$ exponential integral function. 
I( $\Theta)$. The Influence of Concentration on the Diffusion

Coefficient.

As mentioned above, a.II the above theories, w and al. the semi-omplrical equations to be discussed further on, predict the diffusivity of a system at high dilution only.

For systems of concentrations greater than a few percent by weight, the prediction of diffusivity becomes even more confused and unreliable. The influence of concentration on diffusivity is discussed below for

a) non-electrolyties and b) electrolytes. Non-Electrolytes.

For Ideal solutions of non-electrolytes, Powell et aI (20) and willke (2I) propose the relationship

$$
\left(\frac{D, \mu}{T}\right)_{A_{2} B}=x_{A}\left[\left(\frac{D \cdot \mu}{T}\right)_{B O}-\left(\frac{D, \mu}{T}\right)_{A O}\right]+\left(\frac{D \cdot \mu}{T}\right)_{A O}
$$

where $x_{A}=$ mol fraction of $A$ in mixture

suibsexiptis: $A B$, diffusion of $A$ in $B$ at conon. $x_{A}$

$$
\begin{aligned}
& \mathrm{Ao} \text { " "A " } B \text { (very dilute } A \text { ) } \\
& \mathrm{BO}, \quad " \quad \text { " } B \text { "A (very dilute B) }
\end{aligned}
$$

For non-ideal solutions the rtght-hand side of the equation must also be multiplied by an activity correction factox

$$
\text { i.e. }\left(I+\frac{\partial \ln \gamma_{A}}{\partial \ln x_{A}}\right)
$$

where $\gamma_{A}=$ activity of component $A_{0}$ 
Gamex and Marchant (22) on investigating the diffusivities of several associated solutes in water reported that the above relationship gave fairly satisfactory agreement with experimental results.

Caldwell and Babb ( 23 ) investigated three systems which were close to being ideal, and found that equation 52 satisfactorily described the variation in diffusivity with concentration.

Obviously, one limitation of the above relationship is the necessity to have values for the diffusivities at the two concentration extremes.

Unfortunately, apart from the references quoted above, there seems to be Iittle evidence to support the use of the expression.

Caldwelil and Babb also claimed that the group D. $\mu /_{\mathrm{T}}$ valied linearly with mol fraction, which should be the case for ideality. However, inspection of their figures shows that the linearity is not very satisfactory. It would seem that It is not very good practice to correlate the variation in diffusivity with concentration through the group $D_{0} \mu / T^{2}$ even ifthe solutions are assumed to be ideal. Mullin et al ( 24) tried to use this group, with poor results.

Thus, for ideal non-electrolytes, equation 52 appears to be the most adequate for describing the variation in diffusivity with concentration 
Dullien and Shemilt (25) investigated the system ethanol-water over the entire concentration range but did not attempt to use the above equation. Instead they reported the variation in in diffusivity with concentration in terms of the schmidt number $\left(\frac{\mu}{\rho D}\right)$, without any conclusive results.

Strictly speaking, equations 52 and 53 should only be used for diffusion of Iiquid into Iiquid. For the diffusion of solid into liquid (i.e. sucrose-water) the equation proposed by Gordon (26) should be used.

$$
\text { i.e. } D=D_{0}\left(1+\frac{\partial \ln \gamma}{\partial \ln x}\right) \frac{\mu_{0}}{\mu} \cdots .(54)
$$

English and Dole (27) studied the diffusion of sucrose in water in supersaturated solutions, and Gosting and Morris (28) studied the diffusion of sucrose in dilute solutions.

fosting and Morris found that the Gordon relationship was followed and thus proposed that the diffusion of sucrose in dilute solutions was a true diffusional process.

English and Dole showed, however, that as the concentration increased, the agreement between the observed diffusion coefficients and those predicted by the Gordon equation decreased. This they attributed to a) the greater dependence on viscous flow and $b$ ) the influence of hydration on the sucrose molecule. 


\section{Electrolytes}

With concentrated solutions of electrolytes, effects to be considered that will reduce the diffusivity include
1) Thermodynamic effect
2) Electrophoretic effect
3) Viscosity
4) Hydration

In the Nernst-Onsager-Fuoss equation these effects are considered so that corrections should not be necessary.

However, a relationship between $D$ and $D_{0}$ was put forward by stokes et al (29) for concentrated solutions of 1:1 electrolytes

$$
\text { i.e. } \quad \stackrel{\mathrm{D}}{\mathrm{D}}_{0}=\left(1+\mathrm{m} \cdot \frac{\partial \ln \gamma}{\partial \mathrm{m}}\right)(1-0.018 \phi \mathrm{m})\left[1+0.018 \mathrm{~m}\left(\frac{\nu \mathrm{D} *}{\mathrm{D}_{0}}\right)-1 \mathrm{n}\right]
$$

where $m=$ molality

$$
\begin{aligned}
\phi= & \text { hydration number (mols water } / \mathrm{mol} \text { salt }) \\
D *= & \text { self-diffusion coefficient for water } \\
\nu= & \text { no. of ions formed by one stoichiometric } \\
& \text { molecule of salt }
\end{aligned}
$$




\section{$-29-$}

I(f) Semi-Empirical Equations for Predicting the Diffusion

Coefficient

A good review of the semi-empirical correlations available is given by Gambill. (30) and Sherwood and Reid (31).

The correlations are all based on the theories already outlined above and are an attampt to predict the diffusivities of solutions using readily available physical data. It should be noted at this stage that all the main correlations are intended for systems of non-electrolytes, but examination of the data on which these correlations are based reveals that electrolyte systems have been fairly widely used in the data. Attention will be drawn to these cases in the discussions on individual correlations.

Three early correlations that are quite inaccurate are summarised below

Thovert (32)

$$
D \cdot \mu_{B} \cdot \sqrt{M_{\Lambda}}=\text { const. }
$$

Herzog

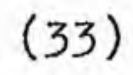

$$
D=\frac{2 R T}{6 \pi N^{0.67}} \mu_{B} \quad\left(v_{A}-b\right)^{0.33}
$$

$\mathrm{b}=$ Van der Vaals volume

$N=$ Avogadros number

Olson and Nalton (34)

Curves are plotted of $D$ against $\frac{\Delta \sigma}{c_{A}}$ with $\sigma$ as parameter ( where $\sigma=$ surface tension and $\frac{\Delta \sigma}{c_{A}}=$ slope of surface tension lowering curve at conen. $c_{A}$ )。 


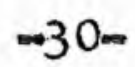

$$
\frac{\Delta \sigma}{c_{A}}=\frac{10^{E / 1318}-I}{0.695}
$$

$$
\text { where } \mathrm{E}=\mathrm{E}_{0}+0.625 \mathrm{n}
$$

$\mathrm{E}_{\mathrm{O}}=$ potential energy contribution of chemical type

(Olson and Walton tabulate values)

$\mathrm{n}=$ number of carbon atoms in molecule

Olson and Walton put forward their correlation on the basis of results given by only 11 organic compounds.

Wilke (21) was the first to make an important step forward in proposing a usable correlation.

Wilke's correlation is derived from the Stokes-Einstein and Eyring theories and employs the use of the group

$$
F=\frac{T}{D} \cdot \mu
$$

$F$ is plotted against solute molal volume, $V_{\Lambda}$, for a number of solutes diffusing in water, benzene, and methyl alcohol. Of the 45 solutes considered diffusing into water, 7 are gases. Of the remainder, almost all exhibit some form of hydrogen bonding or ionisation in solution (examples, - acetamide, acetic acia, acetonitrile)

This immediately makes the correlation and subsequent improvements suspect. Also, as pointed out in the discussion on the stokes-Einstein theory, an activity correction term should be included if the. solutions are not ideal. This has not been done, and thus it i 
is more than likely that errors will be present in the Wilke correlation.

Scheibel (35) expressed the graphical correlation of Wilke by the equation

$$
F=1.22 \times 10^{7}\left\{\frac{V_{A}^{1 / 3}}{1+\left(\frac{3 V_{B}}{V_{A}}\right) / 3}\right\}
$$

or $D=8.2 \times 10^{-8} \cdot \frac{T}{\mu}\left\{\frac{1+\left(\frac{3 V_{B}}{V_{A}}\right)^{2 / 3}}{V_{A} 1 / 3}\right\} \cdots(60)$

From the published data, this equation appears to represent the behaviour of large molecules diffusing through a solvent of small molecules, and appears to be correct for the effect of solvent volume with a solute volume as much as twice the solvent volume.

In the above equation

$$
\begin{aligned}
\mathrm{T} & =\text { absolute temp. } \mathrm{O}_{\mathrm{K}} \\
\mu^{i} & =\text { viscosity of solution, } \mathrm{cp} . \\
\mathrm{V}_{\mathrm{A}}, \mathrm{V}_{\mathrm{B}} & =\text { molecular volume (solute, solvent) }
\end{aligned}
$$

The same provisos as were noted for the Wilke correlation must also apply here. 


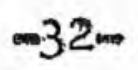

Othmer and Thakar (36)

The correlation is derived from Eyring's theory, using the relationship

$$
\log D=-\frac{E_{0}}{E_{\mu}} \cdot \log \mu+C
$$

Thus a plot of $\log D$ against $\log \mu$ should give a straight Iine.

For solvent water the slope of this line has been computed to have an average value of -1.1 and the correlation

$$
\text { D } \times 10^{5}=\frac{14}{\mu_{\mathrm{W}}^{1.1} \mathrm{~V}_{\mathrm{A}}} 0.6
$$

for diffusion in water has been derived.

For solvents other than water, the general equation for diffusion has been given as

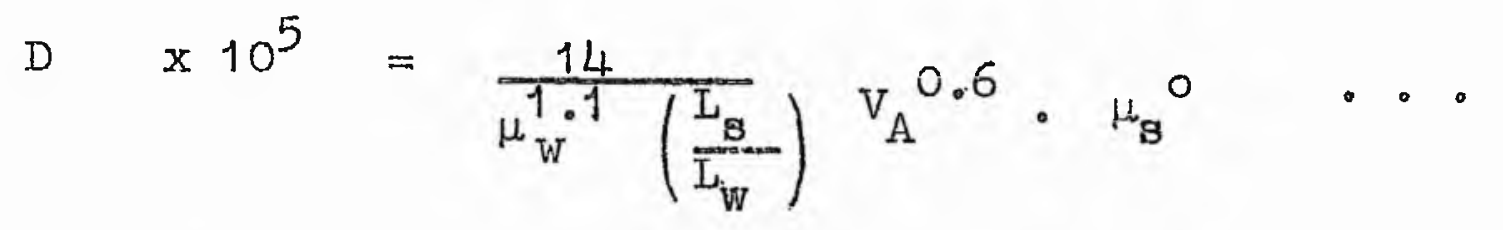

where $I_{s}$ and $I_{w}$ are the latent heats of vaporisation of solvent and water respectively.

$$
\mu_{s}^{o} \quad=\text { Viscosity of solvent. }
$$

Of the 5 solutes diffusing in water considered by othmer and Thakar, one is calcium chloride (a strong electrolyte). The others are :- water (self-diffusing), phenol (a fairly strong acid), mannitol and saccharose (possible hydrogen bonding in solution). Some doubt is thus expressed as to the validity of the correlation. 
Wilke and Chang (37)

This is the most widely used correlation and appears to have found general acceptance. The correlation is a development of the earlier work by Wilke, and is based on diffusion data for a large number of organic liquid systems.

The group $\mathrm{F}=\frac{\mathrm{T}}{\mathrm{D} \mu}$ is again used, being correlated with the solute molal volume, $\mathrm{V}_{\mathrm{A}}$. However, in this correlation the "association parameter" for diffusion in various solvents is considered, - the association parameter, $x$, introduced to define the effective molecular weight of the solvent with respect to the diffusion process.

The correlation for both associated and nos-associated liquids is given by

$$
D=7.4 \times 10^{-8} \quad \frac{(x i M)^{\frac{1}{2}}}{\mu_{0}{ }^{0}{ }^{0}} \frac{. T}{6}
$$

$\begin{array}{ccc}\text { water } & ; & 2.6 \\ \text { methyl alcohol } & ; & 1.9 \\ \text { ethyl alcohol } & ; & 1.5 \\ \text { non-associated liquids } & ; & 1.0\end{array}$

Although an association parameter has been included in the correlation, the same criticisms made of the Wilke correlation will apply. 


\section{$-34-$}

Ibrahim and Kuloor

Subsequent to a diffusional study of the ethanolamines made by Thomas and Furzer (2), Ibrahim and Kuloor (3) proposed two correlations, - partly in an attempt to explain the large differences between the observed diffusion coefficients and those predicted by previous correlations.

The first correlation was a calculation based on gas kinetics, and subsequent communication with Ibrahim (38) has revealed that this was quite irrelevant to any liquidIiquid diffusion case.

The second correlation proposed was subsequently further explained (39), and is based on a modification of Wilke and Chang's correlation. This involves changind $\mathrm{V}^{0.6}$ to $\mathrm{V}^{0.5}$ and including terms for the latent heat of evaporation of the solute and solvent.

Thus

$$
D=5.4 \times 10^{-8}\left[\frac{M_{A}^{\frac{1}{2}} I_{B}^{I / 3} \cdot T}{\mu_{B} V_{A}^{0.5} L_{A}} 0.3\right]^{0.93} \cdot .(65)
$$

115 organic liquid systems were considered. Again, amongst these systems were some such as:acetic acid - water, diethylamine - water etc. and also several gases diffusing into water. There is thus the inevitability that the correlation is based on a large percentage of systems in which a degree of non-ideality is present. 


\section{↔350}

\section{King et al.}

A recent correlation by King et al is based on the observation that $\frac{D \mu}{T}$ is nearly constant for self diffusion. However, the correlation obtained had a $19.5 \%$ standard deviation with their accumulated data. Since this is twice as bad as the correlations of Wilke and Chang etc. then it may be assumed that the correlation is insufficiently accurate to be considered here.

Atomic and Molecular Volumes

In the above correlations, the molecular volumes are calculated from the figures due to Le Bas and given by Wilke and Chang (37). The molecular volume is defined as being the volume, ccs., of one gm.mol, at the normal boiling point.

Atomic Volumes for Complex Molecules ( the molecular volume being additive): $\infty$

Bromine

Chlorine

Iodine

Carbon

Hydrogen

Nitrogen (double bond)

Nitrogen (Iary amine)
27 Nitrogen (2 ary amine)

24.6 oxygen (except as below)

37 oxygen (methyl esters)

14.8 Oxygen (ethyl esters)

3.7

15.6

Oxygen (ethers, other esters)

Oxygen (acids)

10.5 Sulphur
12

7.4

9.1

9.9

11

12

25.6 


$$
-36=
$$

For 3-membered ring, deduct 0.6

$\begin{array}{lcccc}4-" ~ & \text { " } & \text { " } & 8.5 \\ 5-" & \text { " } & \text { " } & 11.5 \\ \text { Pyridine } & \text { " } & \text { " } & 15 \\ \text { Benzene } & \text { " } & \text { " } & 15 \\ \text { Napthalene " } & , & " & 30 \\ \text { Anthracene " } & , & " & 47.5\end{array}$

Molecular Volumes :-

$\begin{array}{llllll}\mathrm{H}_{2} & 14.3 & \mathrm{O}_{2} & 25.6 & \mathrm{~N}_{2} & 31.2\end{array}$

$\begin{array}{llllllll}\text { air } & 29.9 & \mathrm{CO} & 30.7 & \mathrm{CO}_{2} & 34 & \mathrm{SO}_{2} & 44.8\end{array}$

NO $\begin{array}{lllllll}23.6 & \mathrm{D}_{2} \mathrm{O} & 20.0 & \mathrm{~N}_{2} \mathrm{O} & 36.4 & \mathrm{NH}_{3} & 25.8\end{array}$

$\begin{array}{llllllll}\mathrm{H}_{2} \mathrm{O} & 18.9 & \mathrm{COS} & 51.5 & \mathrm{Cl}_{2} & 48.4 & \mathrm{Br}_{2} & 53.3\end{array}$

$I_{2} \quad 71.5$ 


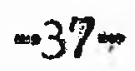

Some Comments on the above Theories and Correlations

Unfortunately, none of the above described theories or correlations are in the least bit satisfactory for general engineering purposes. On the one hand, the theories and correlations for nonmelectrolytes are distinctly hit-and-miss, and on the other hand the theory for electrolytes is too unwieldly for anyone but a pure scientist interested in one system only.

It is as well to quote Wilke and Chang as to the validity of their correlation, since this observation applies equally to all the other equations and puts them in their true perspective.

"... It must be recognised that special structural. features of molecules and other molecular interactions may be important in certain cases and that therefore the proposed relationship (correlation) is at best an oversimplification utilised to obtain a practical result".

When diffusion in the systems ethanolamines-water is discussed later on, with practical results as illustration, it will be seen just how insufficient these correlations are. None of the correlations can be relied upon to predict a D value much better than $10 \%$.

Sherwood and Reid (31) give tables to show calculated and observed values of $D$, using the empirical correlations. The deviations have been calculated for each system. Summaries are given below. 
Diffusion in water

Wilke-Chang Scheibel othmer

Average error \%

11

9

8

Maximum error $\%$

$+30$

$+25$

$+25$

(pyrogaliol)

(alloxan)

$+33$

$+23$

$+23$

(alloxan)

(mannito1)

Diffusion in Methano1

Average error \%

11

12

Maximum error \%

$+32+30$

(chloral hyarate)

$+29+35$

(lactic acid)

Diffusion in Benzene

Average error \%

$\begin{array}{rrr}12 & 10 & 15 \\ +30 & +27 & -30\end{array}$

$\left(\begin{array}{c}\text { chloracetic } \\ \text { acid }\end{array}\right)\left(\begin{array}{l}\text { acetyl } \\ \text { diphenylamine })\end{array}\left(\mathrm{CBr}_{4}\right)\right.$

Again, it will be noticed that in the above systems that produce the maximum deviations, solutes that are ionised or hydrated in solution have been considered. 


\section{$-39=$}

In view of the inclusion of such systems in developing the correlations, it would appear that a far better approach would have been to have developed the correlations using systems that are as near to ideal as possible, and then introduce a term or terms that would account for any deviations due to molecular irregularity etc. However, since the correlations have been developed using irregular systems, then it seems inevitable that large inaccuracies will occur. 


\section{$-40_{-}$}

II THE IXPERIMENTAL DETERMINATION OF DIFEUSTON COIFFICI HINTS

The reviews of the past and present methods for the measurement of the diffusion coefficient are numerous. Amongst the most useful general reviews are those by Tyrel1 (7), Jost (11), Nienow (6), and Johnson and Babb (5). Jost lists a Iurther 30 monographs and review articles (aII pre-1951), so that a general background may easily be obtained.

The measurement of D may be grouped under three headings:-
1) Steady state methods
2) Restricted Diffusion
3) Free Diffusion

\section{II (a) Steady State Methods}

Diffusion is allowed to occur within a column of solution such that a steady (time-invariant) state is set up within the column. Material is supplied to the base and removed from the top, and the concentration at each point within the column remains constant and independent of time. The diffusion coefficient found by this method is the integral coefficient。

Fick, in 1855, used observations from this method to put forward his laws of diffusion. 


\section{$42-$}

Clack (1908) used this method to study diffusion and later improved on accuracy by analysing the solution in the column by refractive index measurements.

Nowadays the most commonly used steady state method is the use of a sintered glass diaphragm cell. In this method, diffusion takes place through a horizontal glass diaphragm separating the two solutions of different composition. Magnetic stirrers are rotated on the upper and lower faces of the diaphragm, and the rate of stirring is such that the diffusion process within the diaphragm may be treated as a steady state phenomenom.

The classical diaphragm cell method is due to Northrop and Anson ( $J_{0} G$ Gen.Physiol. 12. 543.1929), and improvements were made by Stokes ( $(1])$. A good description of a cell and the technique used is given by Dullien and Shemilt (25).

The big disadvantage with the steady state methods is the time factor involved, which may be several days, during which time, of course, the whole system must be kept free of all vibrations and thermal disturbances.

Another disadvantage is that the diffusion coefficients obtained are relative coefficients. The cell has to be calibrated using a known system since there are several unknown factors in the cell, the principal one being the volume of the porous diaphragm.

However, accurate results have been obtained using 
diaphragm cells, particularly the results of Harned and Nuttall (42) who used conductivity measurements to obtain the diffusion coefficient of potassium chloride at low concentrations. These figures are frequently used for the calibration of diaphragm cells.

\section{II (b) Restricted Diffusion.}

A sharp diffusion boundary is allowed to form within the vertical diffusion cell. Mutual diffusion takes place and the concentration on either side of the boundary changes with time. After sufficient time has elapsed the concentration at each end of the cell begins to change, and the process is thereafter called restricted diffusion.

The principle was first described by Graham in 1861, who introduced a concentrated solution beneath a column of more dilute solution. When the concentration had changed throughout the column, layers were drawn off and analysed. The method was improved by subsequent workers, the most important modification being the application of a shearing cell, by means of which a sharp interface could be formed. Disadvantages of the method include the length of time for diffusion (days) and the disruption of the system for analysis. Also, the coefficients obtained by the layer analysis method are strictly neither integral nor differential coefficients o The only accurate results 


\section{$-2+43 m$}

using restricted diffusion appear to be those of Cohen and Bruins ( $\mathrm{z}$, Phys.Chem。103.337. 1923) and Farned and French (Ann,N.Y.Acad,Soc。少。267, 1945)。 Harned and French introduced platinised electrodes into the upper and lower walls of a sheared boundary cell and obtained accurate results for the diffusion of various dilute electrolytes.

\section{II (c) Free Diffusion}

As with restricted diffusion, a sharp diffusion boundary is allowed to form within a vertical diffusion cell. Mutual diffusion takes place and the concentration on either side of the boundary changes with time. It is in the early stages of diffusion, during which time the concentrations at the extremities of the cell are at their initial values, that the process may be termed free diffusion.

Free diffusion measurements are nowadays the prime means of investjgating diffusion, and the analysis of the diffusing system is exclusively optical. One or two workers heve analysed the diffusing column by absorption spectra or by photometry, but nearly all methods now employ the variation in refractive index throughout the column. It wiIl be remembered (equation 16) that the refractive index gradient is proportional to the concentration gradient. It is this basic assumption that is the essential feature of al1 optical methods. 


\section{$-4+4$}

Muller (4.3) gives a comprehensive reviow of optical techniques for the study of diffusional mass transfer, with an extensive list of references (about 100). The review is not quite complete, at least two major developments passing unnoticed, but the best known techniques are well. covered. Tyrell ( 7 ) has also made a good review of optical techntques.

A brief review will be given here of the principal means of optically determining diffuston coefficients, before a detailed description of the interferometer constructed for this work is given. Thus the salient features of these optical techniques may be compared. 
$-45-$ 
U

-

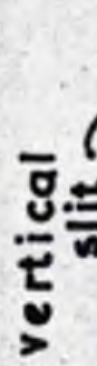

3
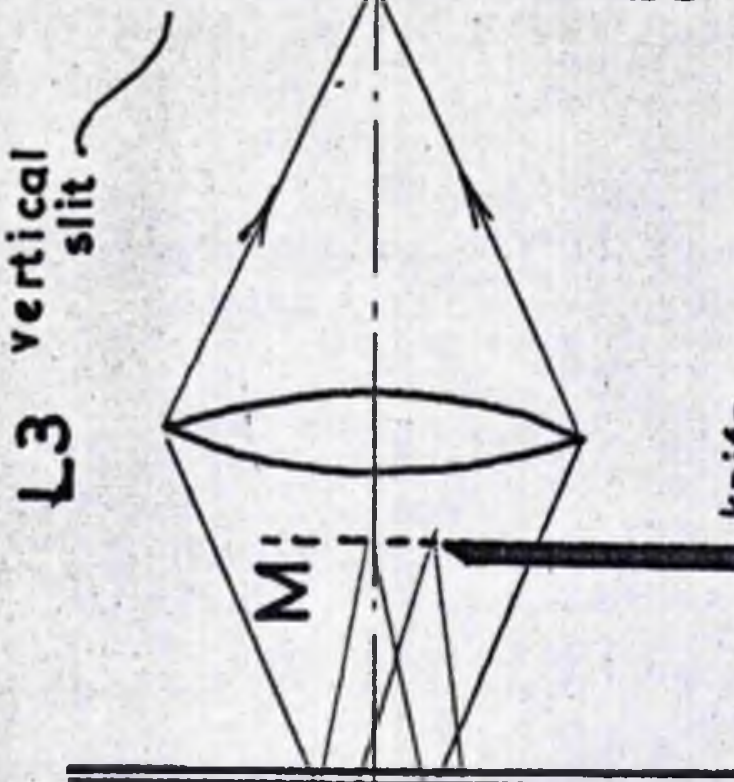

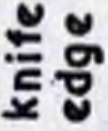

ถัต

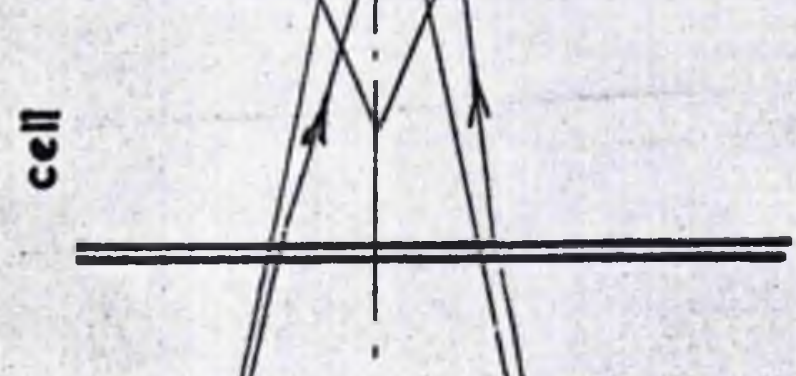

I
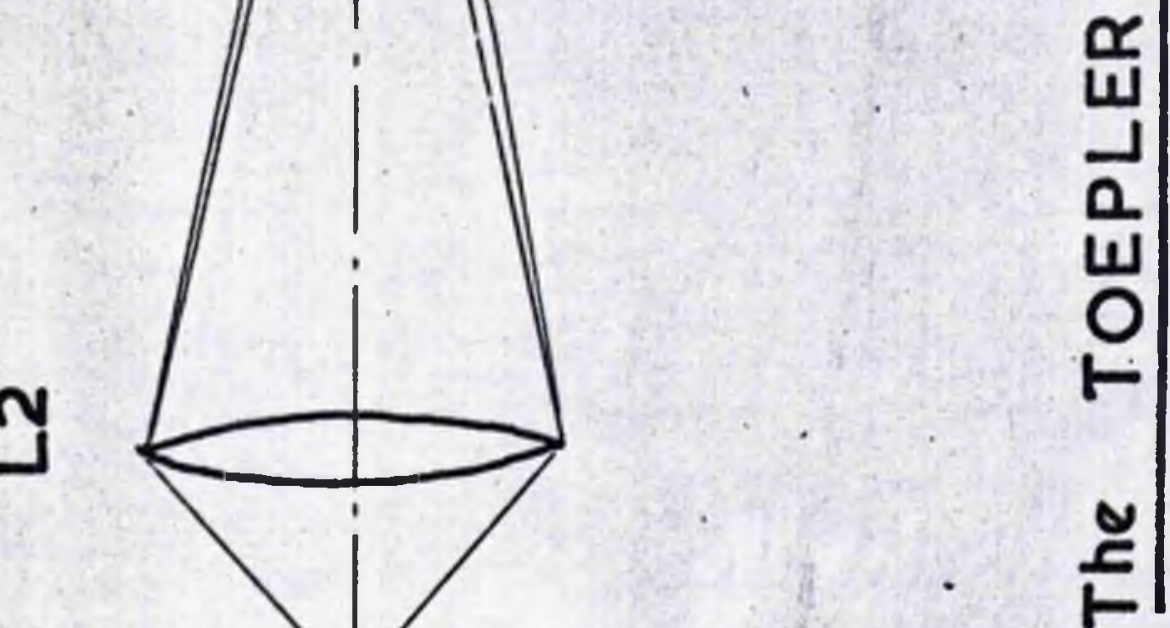

$\frac{\bar{n}}{5}$

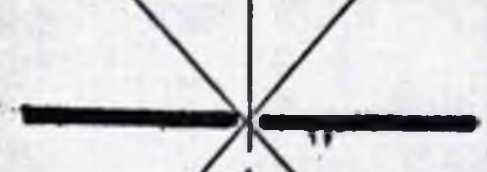

हᄐ
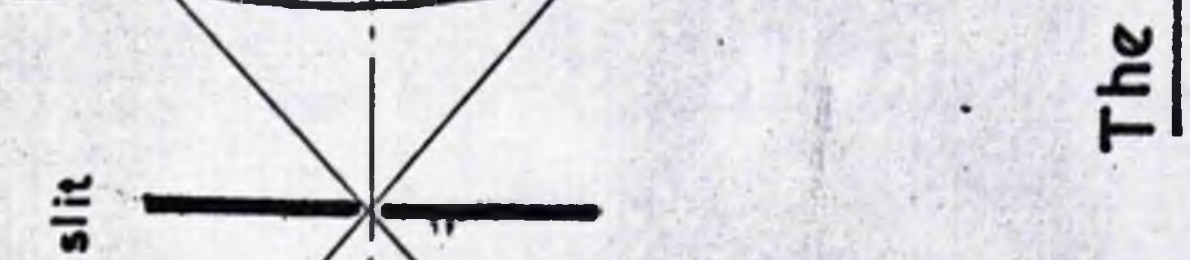

J

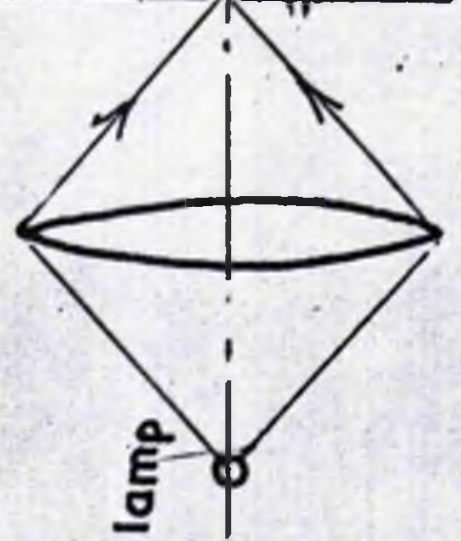

$\frac{0}{1}$ 
II (d) Optical techniques for the study of aiffusional mass transfer.

The optical techniques for the study of diffusional mass transfer may be classified into two groups,

a) Schlieren methods

b) Interferometric methods

Schlieren Methods are based on the curvature of the light path due to the refractive index gradient in the diffusion column. Arrangements are included to transform the angular deflection into an intensity variation, so that a "shadowgraph" record of the refractive index gradient is obtained. The Toepler Scanning Method does this by putting a knife edge in the image plane of the light source, thus preventing all or part of the undeflected light from contributing to the image of the object. The arrangement is show in Fig.1. A photographic plate at the image plane $\mathrm{E}$ is driven horizontally across, whilst the knife edge is moved vertically at a related speed. The recording of a typical concentration gradient obtained by this means is illustrated in Fig.2.

The Philpot-Svennson Diagonal Slit Method is a variation on the Toepler system. A diagonal slit is used instead of the knife-edge; the cell image is focussed in the vertical direction and the slit image is focussed horizontally by a plano-cylindrical lens. 


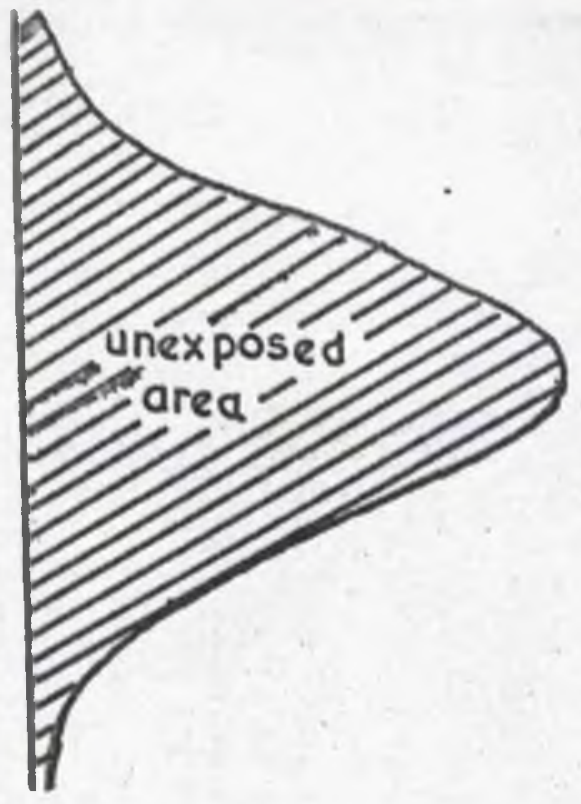

FIG. 2

A Typical Schlieren record of the concentration

gradient during difrusion

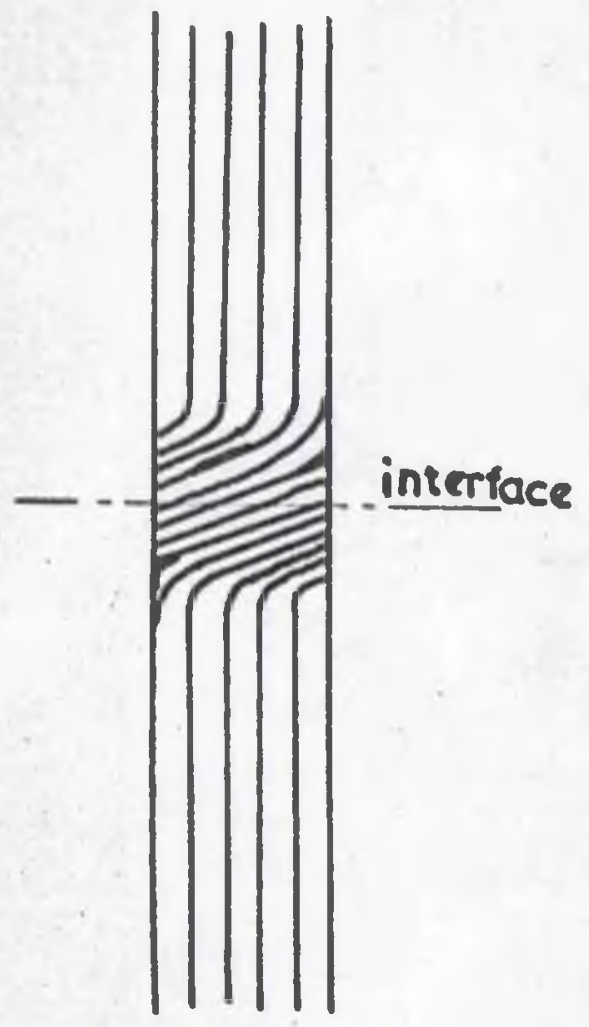

FIG. 3.

Interference fringes as recorded by a Mach Zender during diffusion. 


\section{$2+8 \cdots$}

Interferometric Methods

Interferometric methods have now superseded schlieren methods, since the data obtained is far more readily interpreted and the methods are generally more sensitive.

The basis of interferometric methods is the introduction of a phase difference to the light passing through the cell due to different propagation rates. The phase may be detected by interference with another wave, which may be a reference beam or another part of the same beam.

Since a shift of one interference fringe corresponds to a change in refractive index, $\Delta_{n}$, of $\lambda / a$ (where a $=$ cell length, $\lambda=$ wavelength of light source), which is equal to $5.461 \times 10^{-5}$ for a $1 \mathrm{~cm}$. cell using the mercury green ine, it rollows that interference methods aro very sensitive. It is this that makes interferometry so userul for diffusion measurements.

Numerous interferometers have been used for diffusion measurements, of which the following are listed:Janin; Michelson; Iebedeff; MuItiple Beam; Phase Contrast; Mach Zender; Gouy; Rayleigh; and the Wavefront Shearjing Interferometer (which has been constructed in this laboratory and is the subject of this worl:).

For the purposes of comparison, and for an idea as to the principal interferometers used so far by research workexs in the rield of diffusion, three interferometers 
$-49-$ 


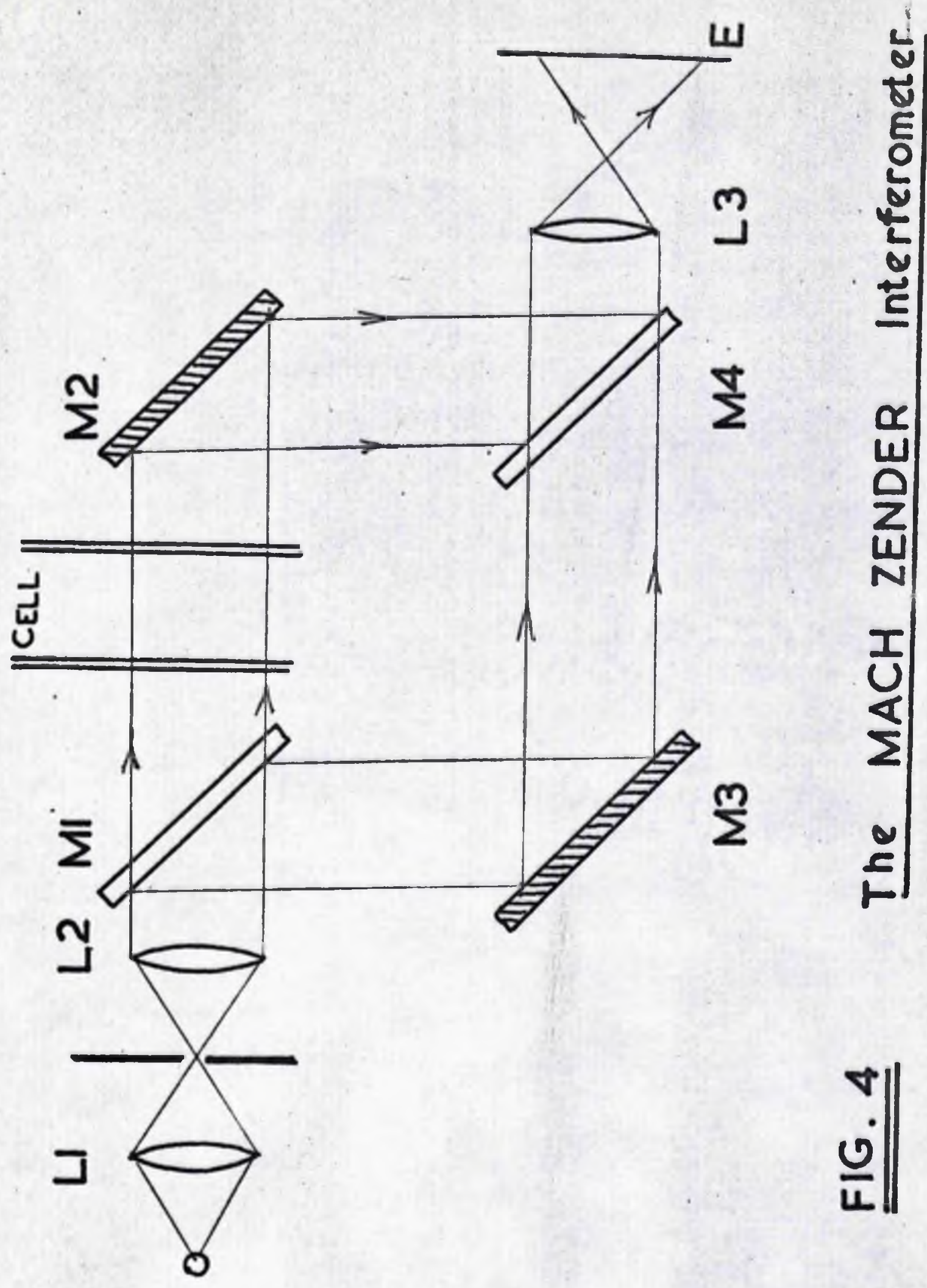


listed above will be briefly described below. They are the Mach Zender, Gouy, and Rayleigh interierometers.

The Mach Zender Interferometer is illustrated in Fig.4. By careful alignment of the mirsors M- - M 4 the light paths are made sufficiently different for interference fringes parallel to the axes of rotation of the fnur mirrors to be produced. A real image of the cell (placed between mirrors $M 1, M 2)$ is formed at plane $\mathbb{S}_{0}$

The fringes produced in the Mach Zender are as in Fig.3. and give the relationship between refractive index of a plane in the diffusion column and its distance from the optic axis.

Amongst the big disadvantages of the Mach Zender is the difficulty in aligning the mirrors, and the high cost (about $215,000)$, since the Mach Zendor is usually bought as a premade unit (construction being difficult for anyone other than an optical expert)。

Descriptions of the Mach zender and its application to diffusion measurements are given by Caldwell et al (45), and Harvey and Smith (46). A description of the instrument may also be had by reading the technical literature supplied by Carl Zeiss and Co.

The sensitivity of the Mach Zender is best illustrated by the results of several workers who have used it. 
a) Caldwell et al (4.5) describe the use of a Mach Zender for determining mutuai diffusion coefficients in binary liquia systems. For calibration a $0.6003 \%$ (by weight) solution of sucrose in water was used. For this, $\Delta n \simeq 7 \times 10^{-4}$.

b) Caldwell and Babb (23) studied the diffusion of ideal binary liquid systems. Examples of concentration differences used, with the weight \% difference in brackets, are:0.022 moI fraction $\mathrm{C}_{6} \mathrm{H}_{6}$ in $\mathrm{CCl}_{4} \quad(1.4 \%)$ $0.033 \quad " \quad{ }_{6}{ }_{6}{ }_{5} \mathrm{Cl}$ in $\mathrm{C}_{6} \mathrm{H}_{5} \mathrm{Br} \quad(2.4 \%)$ $0.0133 \quad " \quad \mathrm{C}_{6} \mathrm{H}_{5} \mathrm{CH}_{3}$ in $\mathrm{C}_{6} \mathrm{H}_{5} \mathrm{Cl} \quad(1.1 \%)$ The above concentration differences correspond to refractive index differences of the order of $1 \mathrm{x}$ $10^{-3}$.

Caldwell and Babb give as their criterion for obtaining workable fringes a figure of $\Delta n=$ $3 \times 10^{-2}$ 。

c) Bidlack and Anderson (47) studied diffusion in ncnideal binary systems. Their concentration differences, and hence $\Delta n^{\prime} s$, were similar to those of Caldwell and Babb.

d) Muli.in et al (24) using a Zeiss diffusion interferometer, which is similar to a Mach Zender, 


\section{$-52 m$}

stated that the maximum and minimum refractive index differences that could be accommodated were $4 \times 10^{-3}$ and $6 \times 10^{-4}$ respectively.

\section{The Gouy Interferometer}

Strictly speaking, the principle of the Gouy interferometer is a hybrid between schlieren effects and interferometry. The Gouy interferometer is illustrat ed in Fig.5, and consists basically and simply of light focussed through the diffusion cell by means of the Biconvex lens I 2. When a concentration gradiont is formed in the cell, the light is bent through progressively greater angles according to the change in refractive index. Since the refractive index gradient is symmetrical about the plane containing the optic axis, planes of the same refractive index gradient are disposed symmetrically above and below this axis. Iight transmitted by the cell in these conjugate planes is brought to a focus at $D$, but the two beams differ in optical path length and their phase difference may be such that destructive or constructive interference occurs. Thus, a set of horizontal fringes is obtained, the total number being proportional to the refractive index difference between the top and bottom of the column. By following the movement of these fringes (having registered them photographically at various time intervals) the diffusion coefficient may be computed. 


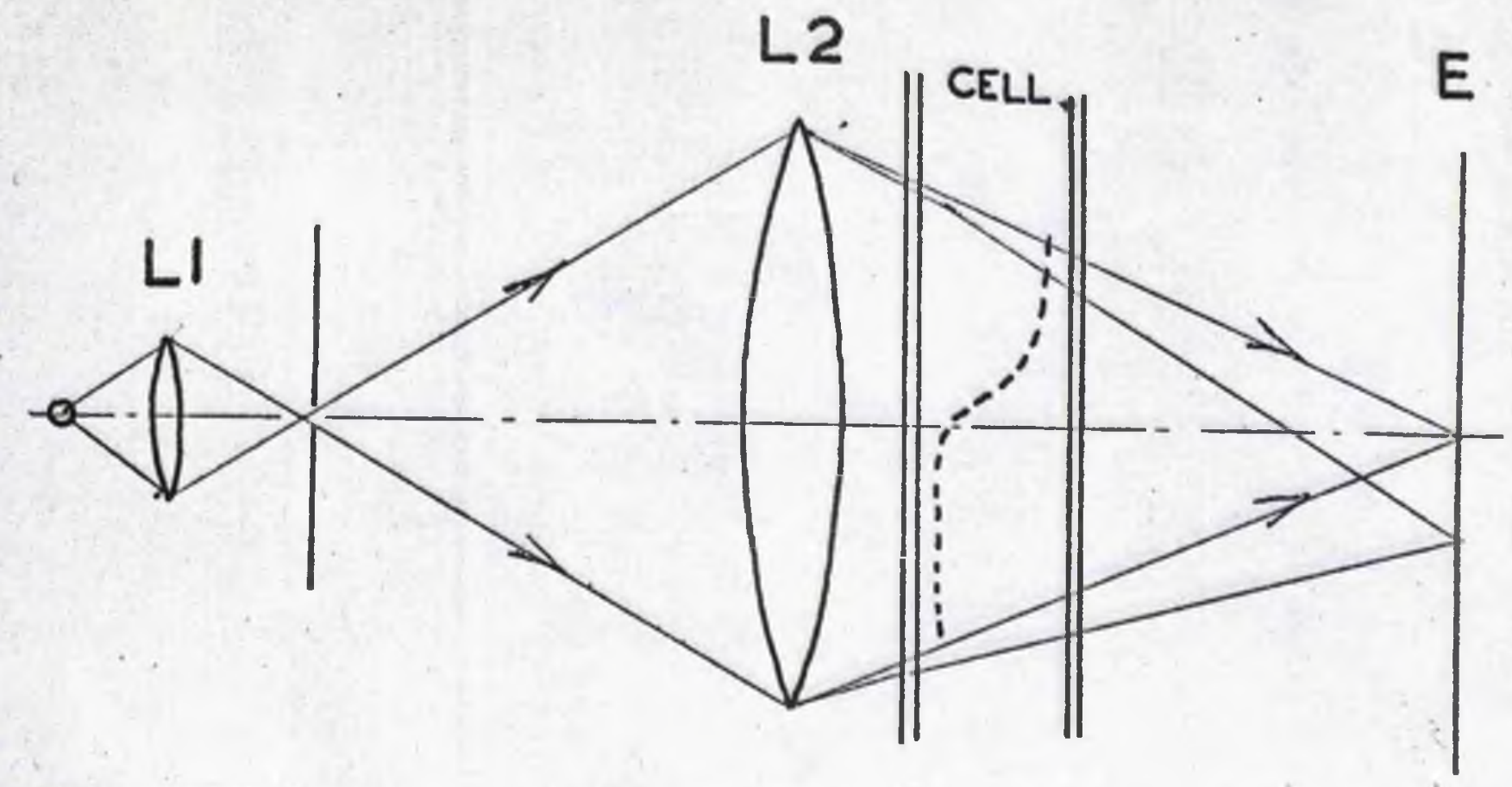

FIG. 5 The GOUY Interferometer

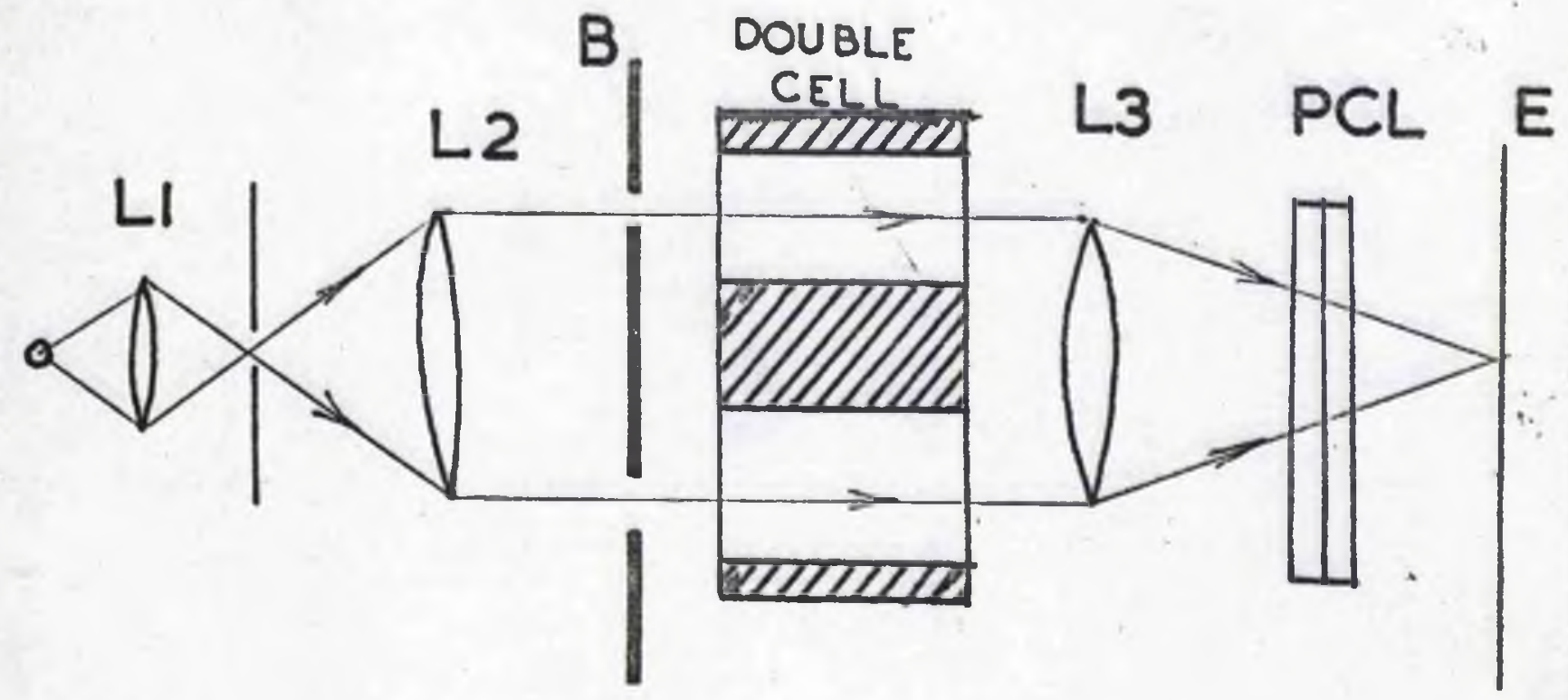

FIG. 6. Plan View of RAYLEIGH

Interferometer. 
Although Gouy described this interferometer in 1880, it was not untiI much later (cirea 1947) that its application was extended to diffusion measurements. Iongsworth (48) was the first to apply the interferometer to diffusional studies and describe them, and in an accompanying paper Kegeles and Gosting (49) developed the necessary theory. Many other workers hare since used the Gouy interferometer, notably so Thomas and Irurzer (1)(2) in this laboratory for a diffusional study of the etinanolamines.

The concentration differences used by Thomas and Furzer were of tho same order as those quoted above for the Wach Zender, i.e. $\Delta n_{\min }$ if $6 \times 10^{-4}$.

The Rayleigh Interferometer is illustrated in Fig.6 (plan view). Parallel light from I2 is passed through a double slit $B$ and the double cell $C_{0}$ I3 focusses the entrance slit $A$ in the horizontal plane, whilst the plano-cylindrical Iens forms a vertical image of $A$ at Hi.

The interference fringes formed during diffusion by this arrangement are similar to those illustrated in tig. 4 for the Mach Zender, and having the same meaning. It is not proposed to give an account here of the optics required for the formation of these fringes (as this would involve quite a bit of detail), but a good account of both the 


\section{0}

theory and the experimental details for the use of the Rayleigh interferometer is givon by svenison (50)(51)(52)。

Typical concentration differences used by Svennson are of the order of 0.5 weight $\%$, with one figure of 0.2 weight $\%$ (for sucrose-water)。 This latter figure corresponds to a $\Delta n \simeq 2 \times 10^{-4}$.

Of necessity, only briaf description, of optical methods for diffusional studies have been given above. It is important to note the concentration differences that are manageable with these interierometers, for although the theoretical sensitivity is given by $\lambda / a$ (refractivo index change), none of these interferometers are operated anywhere near this sensitivity. phis is due almost solely to the lack of optical resolution inherrent in the systems. The theoretical treatment necessary to explain resolution limiting stops is definitely the work of an optical physicist, e.g. Svemson (53). No attempt will be made here at explanation; suffice to say that the resolution is limited in the above described intexperometers.

It is this question of sensitivity that has prompted the cchstruction of a wavefront shearing interferometer in this laboratory, since this system has been shown to differentiate between concentration differences of as low as 0.01 weight $\%\left(\Delta n \approx+\times 10^{-5}\right)$. This interperometer contains no resolution limiting stops. 
This sensitivity is the prime reason for choosing to construct the wavefront shearing interferometer, for not only is the accuracy of the differential diffusion coefficient increased by a decrease in the concentration difference, but the application of interierometry to fields other than Iiquid-IIquid diffusion is immediately suggested (i。e.gas-1iquid difrusion)。

A detailed description of the wavefront shearing interferometer is givon in the next section. 


$$
-57 m
$$

\section{THE WAVE FRONT SHEARING INTERTEROMETRER}

In 1930, Lebedeff (54) described an interferometer which would appear to be the fore-runner of the wave-front shearing interferometer. In the Lebedeff interferometer, polarised light passes through a birefringent crystal plate and is separated into an ordinary and an extraordinaxy ray. These rays then pass through a half wave-plate before being rejoined by a second birefringent crystal plate. The light finally passes through a second polariser.

Lebedeff used this interferometer in conjunction with a microscope to study the growth of crystals. Interference microscopes using this optical arrangement are still in use to-day。

Although the Lebedeft interferometer appears remarkably similar to the wave-wront shearing interferometer, very little reference to it appears to have been made over the past thirty-five years.

The wave-front shearing interferometer was first described by Bryngdanl (4). The theory of the intexferometer is presented by Bryngdahl (55), Bryngdahl and Ijunggren (56) and Ingelstam (57) together with accounts of its basic features. Thomas and Nicholl (58) have described the interferometer, the flowing junction cell used for the diffusional processes, and the means of obtaining diffusion coefficients in binary liquid systems. 
$-58-$ 


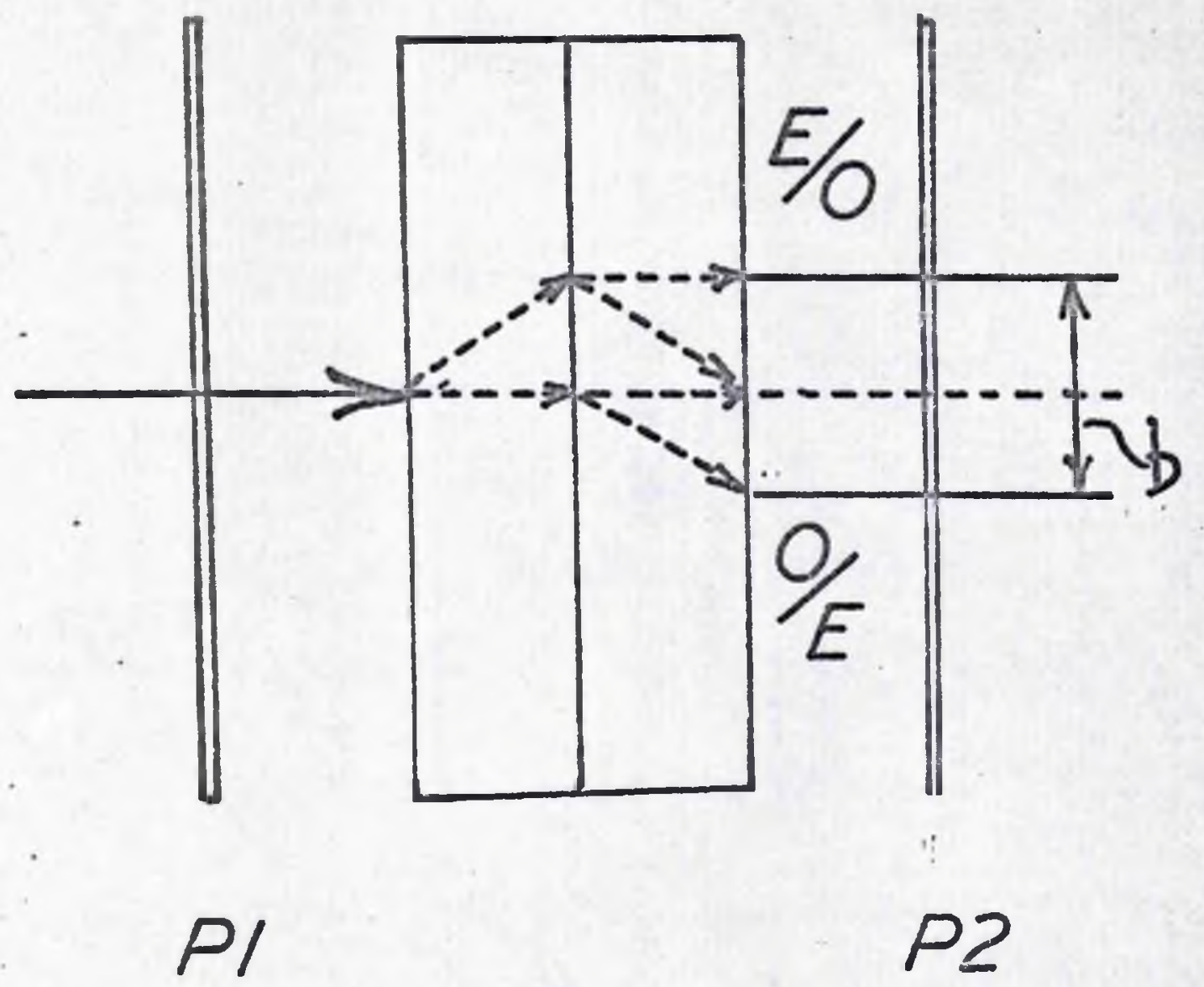

FIG. 7. The SAVART Plate

Showing how the inciant Ifght" ray is split up into an extraordinary/ordinary $(E / O)$ and an ordinary/extraordinary(O/E) ray. $E / O$ and $O / E$ are perpendicularly polarised to each other。 
III (a) Theory

The principle of the waverfront shearing interferometer is the use of polarisod light in conjunction with a savart plate (a birefringent crystal plate). The Savart plate consists of two uniaxial quartz crystals cemented together with their optic axes at $90^{\circ}$ to each other, at $90^{\circ}$ to tho beam direction, and at $45^{\circ}$ with respect to the plane of the paper。(see $\mathrm{Fig}, 7$ )。

Parallel light passes through the first polariser and is polarised such that the oscillation plane bisects the two principal planes of the savart plate. Thus, the wavefront is divided into two equally strong coheront wavefronts polarised perpendicularly to each other. By means of the second polariser, set at $45^{\circ}$ in relation to the principal planes of the crystal plates, the two wetvefironts are caused to interfera.

The displacoment between the wovetronts $(\mathrm{F} / 0$ and $\%$ ) $\mathrm{b}$, is given by the relationship

$$
b=e \sqrt{2} \cdot \frac{n_{e}^{2}-n_{0}^{2}}{n_{e}^{2}+n_{0}^{2}}
$$

where $e=$ thickness of each part of the optical plate

$n_{e^{n}}=$ principal refractive indices 
$-60-$ 

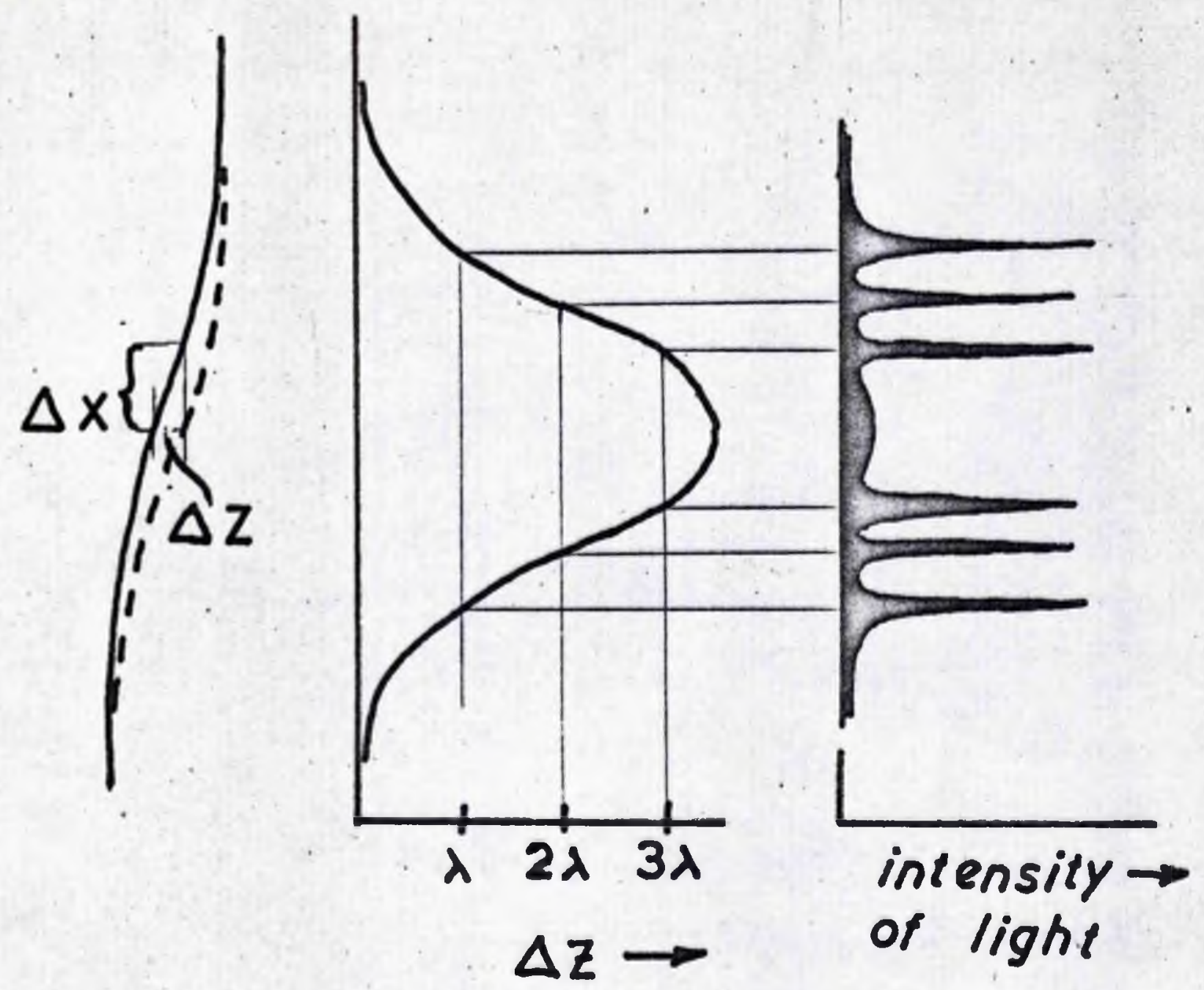

(poth difference)

\section{(interference pottern)}

FIG. 8. Interference Fringe Formation 


\section{$0.07 i=$}

The Savart plates used in this laboratory have e $=10 \mathrm{~mm}$, and $b=84.2 \mu$. If in tho diffusion process to be examined a plane wavefront is passed through a diffusion cell (of rectangular cross section with plane parallel glass windows), the wavefront is distorted in accordance with the concentration of the solution. By passing this aistorted. wavefront through the Savart plate, as described above, the wave-front is split into two parts $(1 / 0$ and $/$ / $)$ separated from each other by a small distance $\Delta x(=b)$ 。

In Fig.8, the two latorelly displaced wave-fronts are shown. If the axes of the polarisers are perpondicular to each other, then interference occurs when the path difference, $\Delta z=\frac{M \lambda}{2} \quad(M=$ whole number

$$
\begin{array}{r}
\lambda=\text { wavelength of light used, } \\
\text { cell length) }
\end{array}
$$

When the derivative curve is so low that $\Delta z<\lambda / 2$, then no fringes are obtained.

The interference patterm produced, a series of horizontal fringe pairs, is related to the Gaussian curve representing the ideal conrentration gradient.

The movement of these fringe pairs is utilisod to calculate the diffusion coefficient. The relationship botween the distance apart of the fringe pairs and time is obtained from the following considerations:-

Before shenring, any one point on the wave-front a 


\section{$-62$.}

distance $x$ from the interface and corresponding to an optical peth length $f(x)$, has a gradient $\Delta \mathrm{s} / \Delta \mathrm{x}$.

After shearing, two points corresponding to the original one are formad a rertical distance $\Delta x$ apart, where

$$
\Delta x=(x+b / 2)-(x-b / 2)
$$

Their corresponding optical path lengths are now

$$
f(x+b / 2) \text { and } f(x-b / 2)
$$

The path difference introduced is given by

$$
z=f(x+b / 2)-f(x-b / 2)
$$

The gradient is given by

$$
\left(\frac{\Delta z}{\Delta x}\right)_{\Delta x=b}=\frac{f(x+b / 2)-f(x-b / 2)}{b}
$$

Referring back to equation (16), which is derived from a solution of Ficks's Second Iaw applickble to the free diffusion case being studied, we have

$$
\frac{\partial n}{\partial x}=\frac{\Delta_{n}}{2 \sqrt{\pi D t}} \cdot \exp \left(x^{2} / 4 D t\right)
$$

Since $z$ is a function of $n$,

$$
\left(\frac{\Delta z}{\Delta x}\right)_{\Delta x=b} \equiv\left(\frac{\Delta n}{\Delta x}\right)_{\Delta x=b}=a
$$

Expanding equation (69), $\left(\frac{\Delta z}{\Delta x}\right)_{\Delta x=b}$, by a Taylor

series (see appenơix) 


$$
\begin{aligned}
\left(\frac{\Delta n}{\Delta x}\right)_{\Delta x=0}=\frac{\partial n}{\partial x}\left\{\begin{array}{l}
1+\frac{1}{2^{2} \cdot 3 !} \cdot \frac{b^{2}}{2 D t} \cdot \frac{x^{2}-2 D t}{2 D t} \\
+\frac{1}{24 !} \cdot \frac{b^{4}}{4 D^{2} t^{2}} \cdot \frac{x^{2}-12 x^{2} D t+12 D^{2} t^{2}}{4 D^{2} t^{2}}
\end{array}\right\}
\end{aligned}
$$

Substituting (16) and (71) into (70), taking the first term in brackets only of $(71)$,

$$
\begin{aligned}
& a=\frac{\Delta n}{2 \sqrt{\pi D t}}\left(1+\frac{b^{2}}{48 D t} \cdot \frac{x^{2}-2 D t}{2 D t}\right) \exp \left(-x^{2} / 4 D t\right) \\
& \text { or } \exp \cdot\left(\frac{x^{2}}{4 D t}\right)=\frac{\Delta n}{2 a \sqrt{\pi D t}}\left(1+\frac{b^{2}}{48 D t} \cdot \frac{x^{2}-2 D t}{2 D t}\right)
\end{aligned}
$$

.$(73)$

If due regard is paid to the value of $t$ in equation (19), a "zero-time correction factor" $\Delta$ t must be introduced, so that

$$
t^{\prime}=t+\Delta t
$$

(true time) (measured time) 
substituting in 16, and rearranginging as above,

$$
\begin{aligned}
& \exp \left(x^{2} / 4 D(t+\Delta t)\right)= \\
& \frac{\Delta n}{2 a \sqrt{(\pi D(t+\Delta t)})} \cdot\left(1+\frac{b^{2}}{48 D t} \cdot \frac{x^{2}-2 D t}{2 D t}\right)
\end{aligned}
$$

Taking logs on both sides and rearranging

$$
\begin{aligned}
& \frac{(2 x)^{2}}{t+\Delta t}= \\
& \quad c-8 D \ln \cdot\left(\frac{t+\Delta t}{t_{0}}\right)+\frac{b^{2}}{3 t} \cdot \frac{x^{2}-2 D t}{2 D t}
\end{aligned}
$$

where $0=8 D\left(1+\ln \cdot \frac{t_{i}}{t_{0}}\right)$

$t_{i}$ is the time of inflexion of the Gaussian curve; $t_{0}$ is an assumed timo unit. In the above reariangement, the assumption $\ln (1+x) \cong x$ has been taken.

$$
\frac{\left(b^{2}\right.}{3 t} \cdot \frac{x^{2}-2 D t}{2 D t} \text { is very small) }
$$

For small At values, (76) may be rearranged to

$$
\begin{gathered}
\frac{(2 x)^{2}}{t}\left(1-\frac{\Delta t}{t}\right)=c-8 D \ln \frac{t}{t_{0}}-8 D \frac{\Delta t}{t}+ \\
\frac{b^{2}}{3 t} \cdot \frac{x^{2}-2 D t}{2 D t \ldots \ldots \ldots o(77)}
\end{gathered}
$$

Simplifying,

$$
\begin{aligned}
\frac{(2 x)^{2}}{t}=c-8 D \ln \cdot \frac{t}{t_{0}}+ & \frac{1}{t}\left(\frac{(2 x)^{2}}{t}-8 D\right) \\
& \left(\frac{b^{2}}{24 D}+\Delta t\right)
\end{aligned}
$$


Putting $\left.\begin{array}{rl}\phi & =(2 \mathrm{x})^{2} / t \\ \phi & =\ln t / t_{0}\end{array}\right\}$ we get

$$
\eta=0-8 D \phi+\frac{\eta-8 D}{t} \cdot\left(\frac{b^{2}}{24 D}+\Delta t_{e}\right)
$$

Thus, the deviation caused by $\Delta \mathrm{n} / \Delta \mathrm{x}$ in the first approximation, appears as a constant contribution to the zero time correction.

$$
\begin{aligned}
\Delta t_{\mathrm{e}} & =\Delta t_{\mathrm{W}}+\mathrm{b}^{2} / 24 \mathrm{D} \\
& (e=\text { apparent, } \mathrm{w}=\text { real })
\end{aligned}
$$

Whon the correct zero time correction is applied to (79) the equation renresenting the movement; of the fringe pairs reduces to

$$
7=\mathrm{C}-8 \mathrm{D} \phi
$$

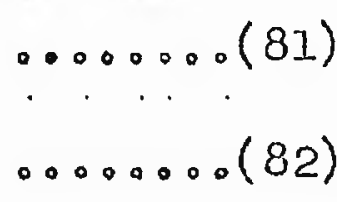

or $(2 x)^{2}=8 D t \cdot\left(1+\ln \frac{t_{i}}{t}\right)$

If therefore, the distance apart of any one fringe pair, $(2 x)$, is followed with respect to time, $t$, the dirfusion ecerficiunt may be obtained. There are several methods of calculation, all of them determining both the zero time correction factor, $\Delta t$, and the diffusion coefficient. The zero-tine correction factor is vexy important, since it has quite a significant effect on the diffusion coefficient. In practice, it is impossible to obtain an 
$-66$

initially sharp interface, since there is always some initial mass transfer between solutions. This has the effect of displacing a plot of $(2 x)$ against $t$ towards the left.

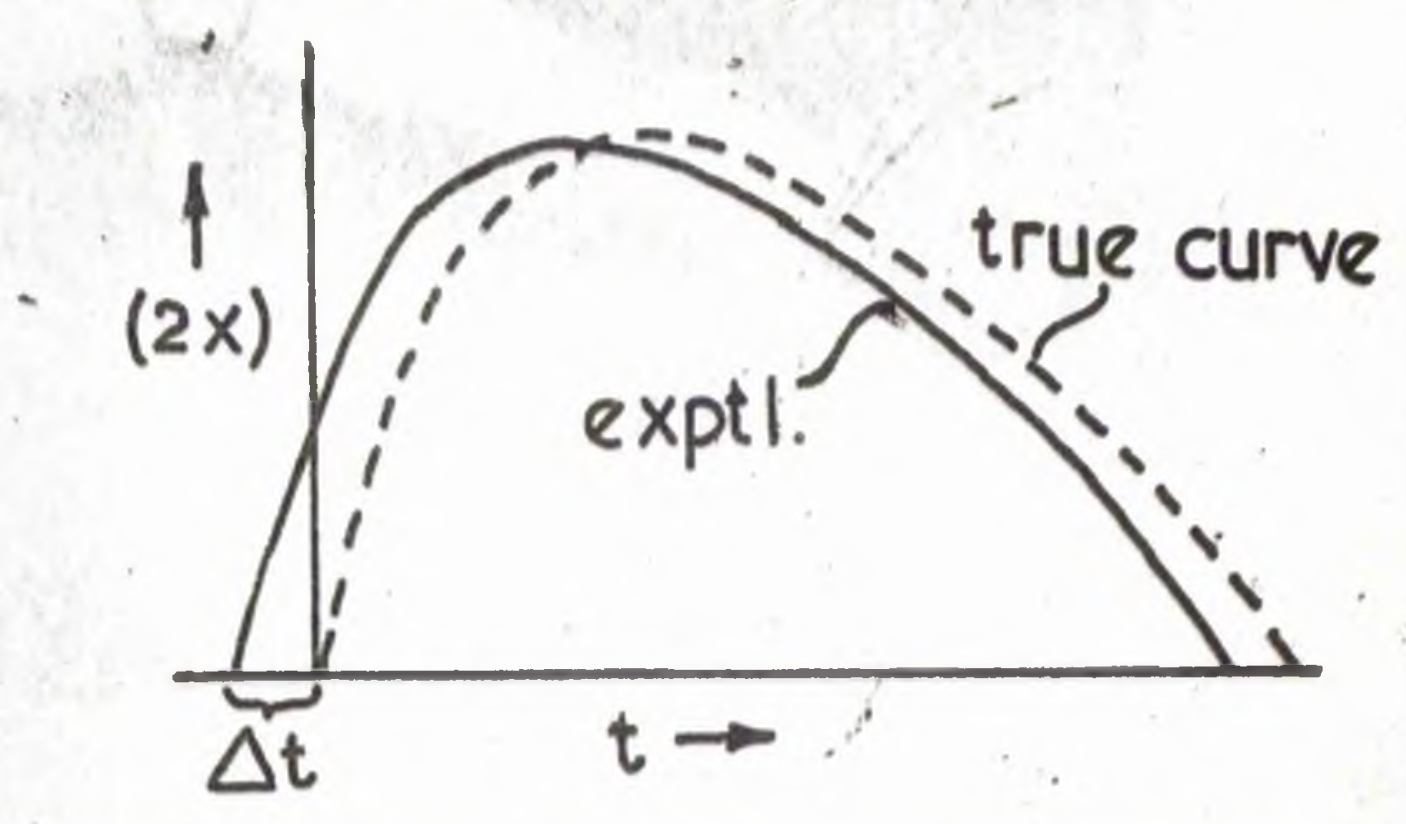

It is this displacement, $\Delta t$, that has to be determined before the true $D$ may be calculated.

The methods of calculation are discussed below.

III (b) CaIculation of $D_{\text {, and }} \Delta_{t}$

The first four methods to be described are due to Bryngdahl. The fifth method is entirely new, and has been developed in this laboratory. 
(i) From equation (82) the relationship

$$
\frac{(2 x)^{2}}{t}=C^{\prime}-8 \operatorname{DIn} \frac{t_{j}}{t} \quad \text { may be derived. }
$$

If $\frac{(2 x)^{2}}{t}$ is plotted against $\mathrm{ln} t$ for any one fringe pair, repeatedly adding a small time increment to the $t$-values before plotting the curve, then, theoretically, at the stage when the correct $\lambda t$ has been applied the curve should be a straight line of slope - 8D. In practice it has been found that the method is very insensitive, and is not sufficiently accurate to be of any use.

(ii) In runs where more than one fringe pair is observed and recorded, these are plotted on a graph of $(2 x)^{2}$ against to A straight line is drawn connecting the maxima of the curves. This line is of slope $8 D$, and from the point where it cuts the t-axis, $\Delta t$ may be determined. This method, again, is limited in accuracy, and only gives approximate results。

(iii) In a method of calculation due to Lamm, from a plot of $(c x)^{2}$ against $t$ for any one fringe pair $D$ may be, calculated from the expression

$$
D=\frac{(2 x)^{2}\left(\frac{1}{t_{1}}-\frac{1}{t_{2}}\right)}{8 \ln \frac{t_{2}}{t_{1}}}
$$


Here, $t_{1}$ and $t_{2}$ are two time-values corresponding to any one value of $(2 x)^{2}$. However, Bryngdahl has shown that the true $D$ is related to the calculated $D$, $D^{\prime}$, by the expression

$$
D=\frac{D^{\prime}}{i+\Delta t \cdot 2 r}
$$

where $\nu=\frac{t_{1}+t_{2}}{t_{1} t_{2}}-\frac{1 / t_{1}-1 / t_{2}}{\ln t_{2 / t_{1}}}$

If, then, $D$ is calculated for any one value of $(2 x)^{2}$ and the corresponding $t_{1}$ and $t_{2}$ values using equation (83), and plotted against $V$, a straight line is obtained of slope D. At, cutting the ordinate axis at $D$. Using this method $D$ may be calculated to 3 significant pigures (i.e. $5.21 \mathrm{x}$ $\left.10^{-6} \mathrm{~cm}^{2} / \mathrm{sec}_{0}\right)$. It is not, therefore, all that accurate, and it was round more convenient in practice to only use this method as a check before using the fourth method.

(iv) The fourth method of calculation is the most accurate of the methods mentioned so far. $D$ is calculated by equation (83), and is plotted against $(2 x)^{2}$, with $\Lambda t$ as a parameter. This is done by first calculating $D$ with no correction applied to the time, for a set of values of $(2 x)^{2}, t_{1}, t_{2}$ Then a small time-increment is added to the t-values and the calculations are repeated. This is 
continued until the value of $D$ becomes constant when plotted against $(2 x)^{2}$. At this point, the correct value of $D$ is obtained, together with the zero-time correction, i.e.

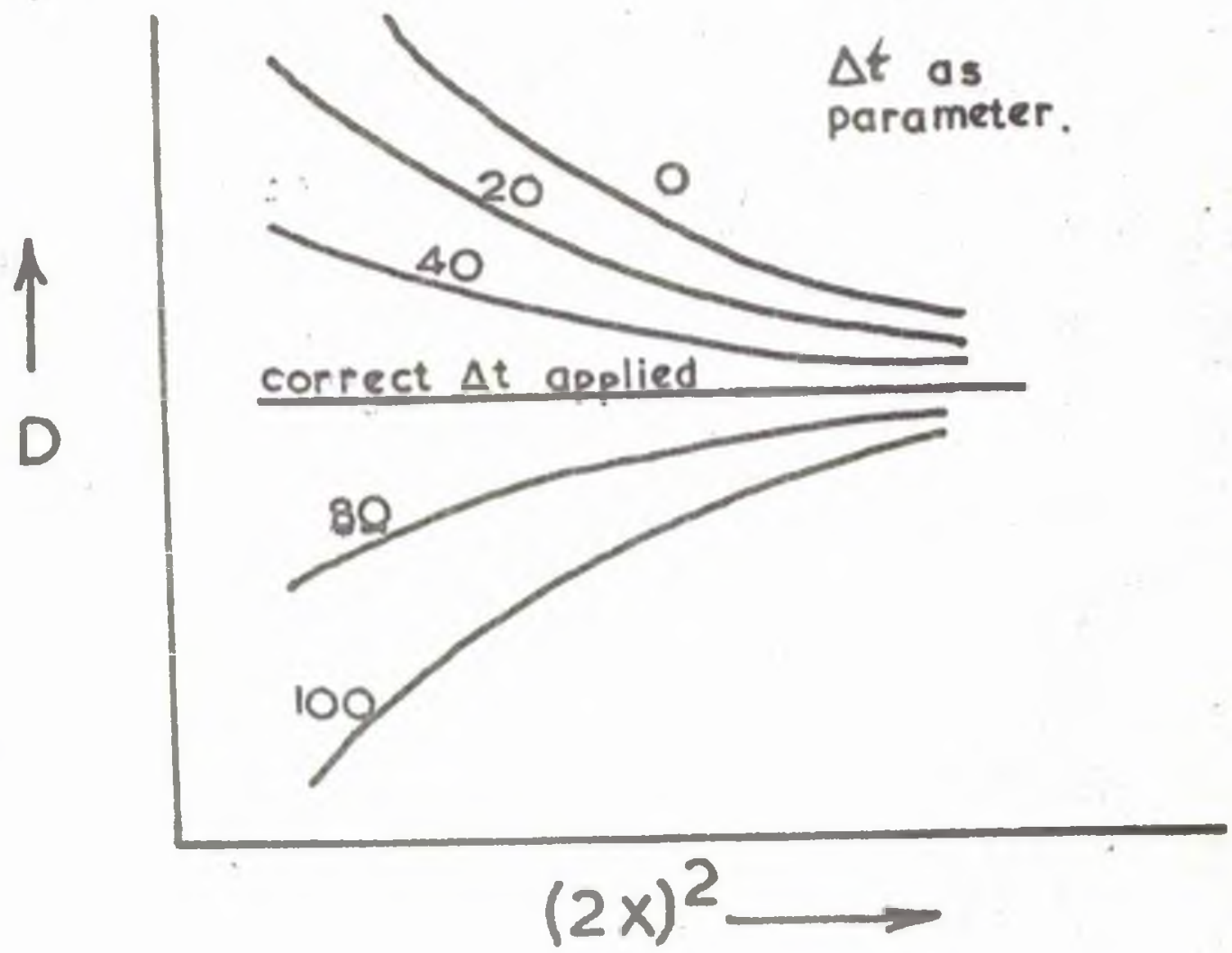

By this method, D may be calculatsd to four significant figures.

A fault common to all the four methods outlined above, is that the accuracy of the calculation is dependant upon readings taken from a smooth curve drawn through the experimental points on $a(2 x)^{2}$ vs. $t$ plot. The method developed in this laboratory, overcomes this difficulty. It thus becomes less sensitive to error than any of the above methods, especially when a slight scatter of points 


\section{$-70 m$}

on the experimental curve of $(2 x)^{2}$ vs. $t$ cannot be avoided. In practice, it has also become apparent that this new method is much more convenient to use, and is highlyr accurate (so that the accuracy of the determination of $D$ is Iimited by the apparatus rather than the calculation).

III (C) New Method for Calculating D

The method of calculation uses equation (82) in which the movement of one fringe-paix with respect to time is represented. The feature of the calculation, however, is the use of the method of least squares. The sequence of steps in the method will now be described.

\section{Least Squares Curve Fitting}

Consider the Iinear equations

$$
\begin{aligned}
& \mathrm{y}_{1}=\left(a \mathrm{x}_{1}\right)_{1}+\left(\mathrm{bx}_{2}\right)_{1}+\ldots \ldots+\left(\mathrm{gx}_{\mathrm{k}}\right)_{1} \\
& \mathrm{y}_{\mathrm{n}}=\left(\mathrm{ax}_{1}\right)_{\mathrm{n}}+\left(\mathrm{bx}_{2}\right)_{n}+\ldots \ldots+\left(\mathrm{gx}_{\mathrm{k}}\right)_{n}
\end{aligned}
$$

in which there are ' $n$ ' sets of observations of $y$ and $x$.

( $y$ values are dependant variables, $x$ values are independant variables, $a, b, \ldots \ldots, z$ values are onstants: If the true values of $a, b, \ldots \ldots$, are $A, B, \ldots \ldots$, the true values of $\mathrm{y}_{1}, \mathrm{y}_{2}, \ldots \ldots$ are $\mathrm{Y}_{1}, \mathrm{Y}_{2}, \ldots \ldots$, and the differences between the true and observed values of $y$ are $\phi_{1}, \phi_{2} \ldots$. then 


$$
\begin{aligned}
\phi_{1} & =Y_{1}-y_{1} \\
& =\left(A x_{1}\right)_{1}+\left(B x_{2}\right)_{1}+\ldots \ldots+\left(B x_{k}\right)_{1}-y_{1} \\
\phi_{2} & =Y_{2}-y_{2} \\
& =\left(A x_{1}\right)_{2}+\left(B x_{2}\right)_{2}+\cdots \cdots+\left(B x_{k}\right)_{2}-Y_{2}
\end{aligned}
$$

Then, $\phi_{1}, \phi_{2}$ are exrors whose chances are given by a normal distribution curve. The assignment of values to $a, b, \ldots \ldots$ to minimise the inconsistency which is assumed to be due to imperfect measurement of the ' $\mathrm{x}$ ' values is obtained by finding those values of $A, B, \ldots .$. which make $\phi_{1}^{2}+\phi_{2}^{2}+$ .... a minimum. The conditions for this are that

$$
\frac{f\left(\phi_{1}, \phi_{2} \ldots\right)}{A}=\frac{f\left(\phi_{1}, \phi_{2} \ldots\right)}{B}=0
$$

It may be shown that the normal equations satisfying this condition (for $k=2$ ), are given by

$$
\left.\begin{array}{l}
A \sum x_{1}{ }^{2}+B \sum x_{1} x_{2}=\sum x_{1} y \\
A \sum x_{1} x_{2}+B \sum x_{2}^{2}=\sum x_{2} y
\end{array}\right\}
$$

whose simultaneous solution cnables $A, B$, to be found. The simultaneous solution of two such equations is most easily done by use of a matrix of the form

$$
\left[\begin{array}{l}
\sum x_{2} y \\
\sum x_{1} y
\end{array}\right]=\left[\begin{array}{ll}
A \\
B
\end{array}\right]\left[\begin{array}{ll}
\sum x_{2}^{2} & \sum x_{1} x_{2} \\
\sum x_{1} x_{2} & \sum x_{1}^{2}
\end{array}\right] \ldots \ldots(90)
$$




\section{$-72$}

$\operatorname{or}[\mathrm{Y}]=[\mathrm{C}][\mathrm{V}]$

so that $[\mathrm{C}]=[\mathrm{Y}][\mathrm{V}]^{-1}$

When a set of observations of $(2 x)^{2}$ against $t$ are made to satisfy equation (82), the $\mathrm{x}, \mathrm{y}$ values and constants in (90) will correspond as below (including $\Delta t$ in time measurenents)。

$$
\begin{aligned}
& y:(2 x)^{2} \\
& x_{1}: 8(t+\Delta t)-8(t+\Delta t) \ln (t+\Delta t) \\
& x_{2}: 8(t+\Delta t) 。 \\
& A \quad: D \\
& B \quad: D \ln t_{i}
\end{aligned}
$$

Using appropriate matrix subroutines (to satisfy equation (91)), a computer may be programmed to calculate. the best $D$ and $t_{i}$ to fit a set of experimental values of $(2 x)^{2}$ and to In this laboratory, a Ferranti sirjus computer was used and details of the calculation are given below.

Application of the Method of Least Squares to Calcuiate $D$

When no $\Delta t$ is applied to the experimental points, it will be remembered that a true Gaussian curve draw through these points cuts the t-axis to the left of the origin. This displacement in $t$ is equivalent to the zero-time correction, $\Delta t$. 
However, when solving the normal equations for a set of $(2 x)^{2}, t$-values, the point $(0,0)$ is automatically included. The least squares fit does not take into account any displacement of the experimental points. Thus, the curve of

$$
(2 x)^{2}=8 D t\left(1+\ln \frac{t_{i}}{t}\right) \quad \text { (equation 82) }
$$

as calculated from the best fitting $D$ and $t_{i}$ for the experimental points (using the method of least squares), and hercafter referred to as the Regression Curve of $(2 x)^{2}$ on $t$, will be as with curve $A$, below:-

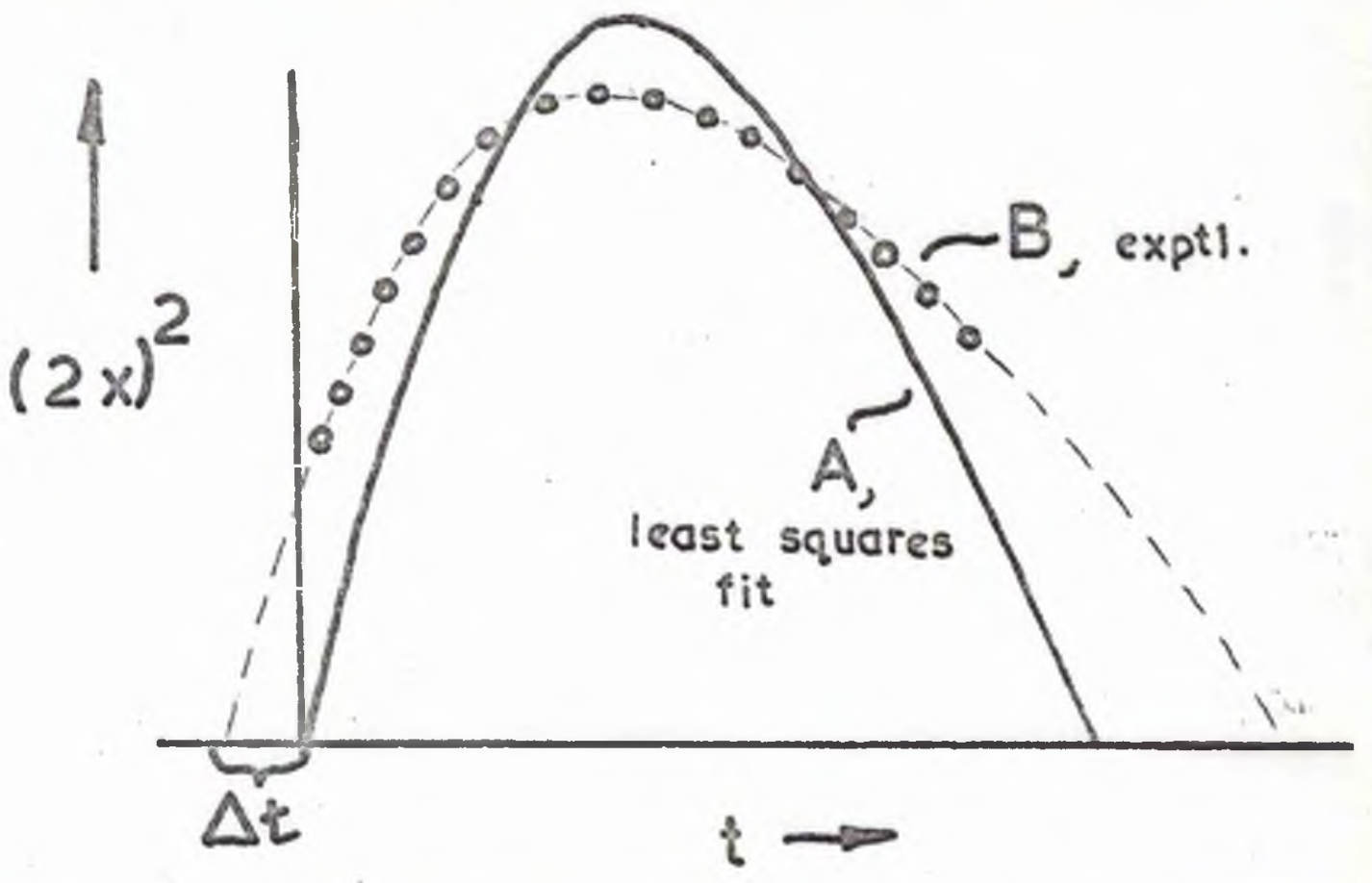

With no $\Delta t$ applied to the experimental points, the regression curve $(A)$ of $(2 x)^{2}$ on $t$ has a greater "peakiness" to it, as above. 


\section{$\infty 74=$}

If now a $\Lambda t$ is applied, thus effectively shifting the experimental points to the right, the regression curve willnot be as "peaked" as originally,

As repeated $\Delta t$ increments are added to the experimental time values, the agreement in shape and position of the regression curve and the experimental points becomes closer. iventually, when the correct At has been applied to the experimental points, the two curves are coinciaental.

If too great a At is applied to the experimental points, it will be seen that the regression curve becomes less peaked than the experimental curve.

Thus, a criterion must be established whereby the agrement between the regression curve and the experimental points is judged to be best. At this point, the correct $\Delta t$ has been applied to the experimental points, and hence the D-value may be obtained.

A simple criterion is to compara the maxima of the two curves. The maximum of the experinental curve, $(2 x)_{i . B}^{2}$, is found from the experimental points. The maximum of the regression curve, $(2 x)_{i A}^{2}$ is given by the equation

$$
(2 x)_{i}^{2}=8 D t_{i}
$$

Mquation (92) is derived from (32) by putting $t=t_{i}$ 。 
$-7500$

When $t_{\text {applied }}<t_{\text {true }},(2 x)_{i, A}^{2}>(2 x)_{i B}^{2}$

$$
\begin{aligned}
& t_{\text {applied }}>t_{\text {true }},(2 x)_{i A}^{2}<(2 x)_{i B}^{2} \\
& t_{\text {applied }}=t_{\text {true }},(2 x)_{i A}^{2}=(2 x)_{i B}^{2}
\end{aligned}
$$

Thus, a print-out of $(2 x)_{i A}^{2}$ on the computer output will enable a comparison of the two $(2 x)_{i}^{2}$ values, so that when they are equal it may be judged that the correct $\Delta$ t has been applied.

A more accurate criterion is to calculate the scatter of the experimental points from the least squares regression curve of $(2 x)^{2}$ on to This scatter is termed the standard Irror of Isistimate of $(2 x)^{2}$ on $t$, and is defined by

$$
s=\sqrt{\sum_{1}\left[\frac{(2 x)_{B}^{2}-(2 x)_{A}^{2}}{N}\right]^{2}}
$$

Thus, for each $\Delta t$ value applied to the experimental points, the standard error of estimate, $\mathrm{S}$, between the experimental points and the regression curve of $(2 x)^{2}$ on $t$ is calculated. As the $\Delta t$ is increased, $s$ decreases until a minimum value is reached. At this minimum, the two curves are in agreement, and thus it may be judged that the correct $\Delta t$ has been applied.

The correct $D$ is thus found, since the $D$ calculated by the method of least squares for the experimental points, is the true $D$ when the correct $\Delta t$ has been applied. 
$-76-$ 


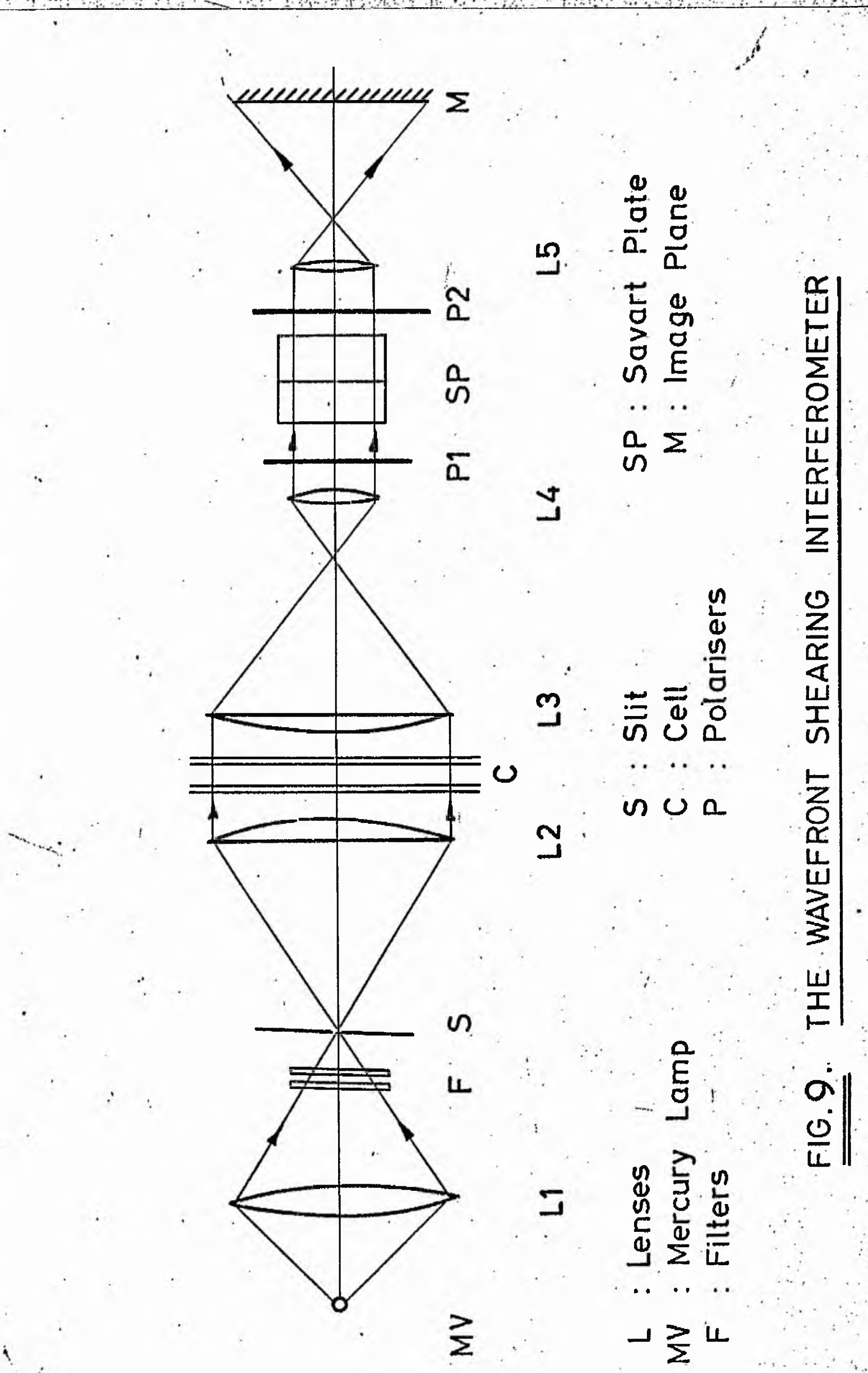




$$
\infty 77 \%
$$

This method of calculation is illustrated in the section dealing with the experimental results。

This method of calculation gives an exact value of $D$, and is accurate to four significant figures (e.g. $5.221 \mathrm{x}$ $10^{-6} \mathrm{~cm}^{2} / \mathrm{sec}$ ). The method has been repeatediy used in this laboratory, and is very convenient in use. It is essential of course, if this method of calculation is to be wholly successful, that the experimental points are fairiy complete and that they are "weighted" equally to either side of the maximum. However, this condition applies equally well to any other method of calculation. The true advantage of the method is that a slight scatter of experimental points is permisstble, without deterring from the accuracy of the calculation.

\section{III (d) Apraratus}

The arrangement of the wavefront shearing interferomeier is shown in Fig.9. The complete optical system is contained in a constant--temperature (thermostatically controlied) darkroom. The tomperature of the room can be maintained to $\pm 0.1^{\circ} \mathrm{C}$ (most experiments being conducted at $\left.25^{\circ} \mathrm{C}\right)$

The optical components are mounted on a 3-metre long optical bench, of triangular section, resting on two 10-it Iong steel girders bolted to three concrete pillars. The 


\section{$-78$}

whole assembly, weighing over 1000 tbs, is separated from the floor by rubber biocks. The various components in the optical system are attached to the bench by adjustable saddle stands, fitted with clanping screws.

A 250-W high pressure mercury vapour lamp (Miv), operated in a vextical position provides a cource of monochromatic light (at $5461 \AA$ ). The narrow discharge from the lamp is focussed through a short focal length condensing lens, L1, through infrared and green filters on to a horizontal adjustable slit operated from $5 \mu$ to $10 \mu$.

To reduce the effect of stray light, the lamp, condenser, filtexs and slit are housed in a light-proof structure (which is adequately ventilated).

By means of the plano-convex lens I2, parallel light is passed through the diffusion cell, C. By I3 and IL, the image from the cell is collimated through the savart Plate (SP). Polarisers, P1 and P2, are placed before and after the Savart plate. The image formed after the Savart plate is rather small, and it is convenient to magnify the final image by means of the lens $\mathrm{L} 5$ a

Detajls of the lenses used are given below:-

I1 : Biconvex condenser Iens, $100 \mathrm{~mm}$ dia. $F=100 \mathrm{~mm}$ 。

L2,I3: Plano-convex lenses, $102 \mathrm{~mm}$ dia, Corrected Por spherical and chromatic aberrations. 
IL,I5: Biconvex lens, $20 \mathrm{~mm}$ dia。 $f=75 \mathrm{~mm}$

The diffusion occurring in the cell. with the resultant interference fringes, is recorded photographically in image plane M. The camera used is a $35 \mathrm{~mm}$ Robot-Recorder camera mounted on the bench with a Robot Kuli Universal camera holder. The camera is ritted with a 30-ft film magazine, and an electric booster motor (for continuous wind-free transportation of the film). The camera is coupled to a steuergerat IIa electromechanical time-control set. By means of this set, the canera exposure and the interval between exposures may be set anywhere in the range 0.15 secs to $3 \mathrm{hrs}$. The film used is Kodak $R .55$ recording film, developed in DX-80 developer ( $2 \frac{1}{2}$ minutes development at $24^{\circ} \mathrm{C}, 1+3$ ailution).

R.55 recording rilm was chosen after trials with other high speed silm (i,e. Ilford HP3, Kodak Tri-X), since it has both a high speed and a maximum sensitivity to light between $5400 \AA$ and $5700 \AA$ (59). The use of $R .55$ film is further enhanced by its insensitivity to red light, thus making its handling very simple (the film may be loaded and. developed in light from a Kodak Wratten $1 \mathrm{~A}$ filter, with a 15-w bu.1b)。

The Diffusion cell was constructed by Furzer (1) in this laboratory, and is of the flowing-junction type. The cell is shown in Fig.10 (photograph) and the principle of its construction and operation is diagrematically shown in 


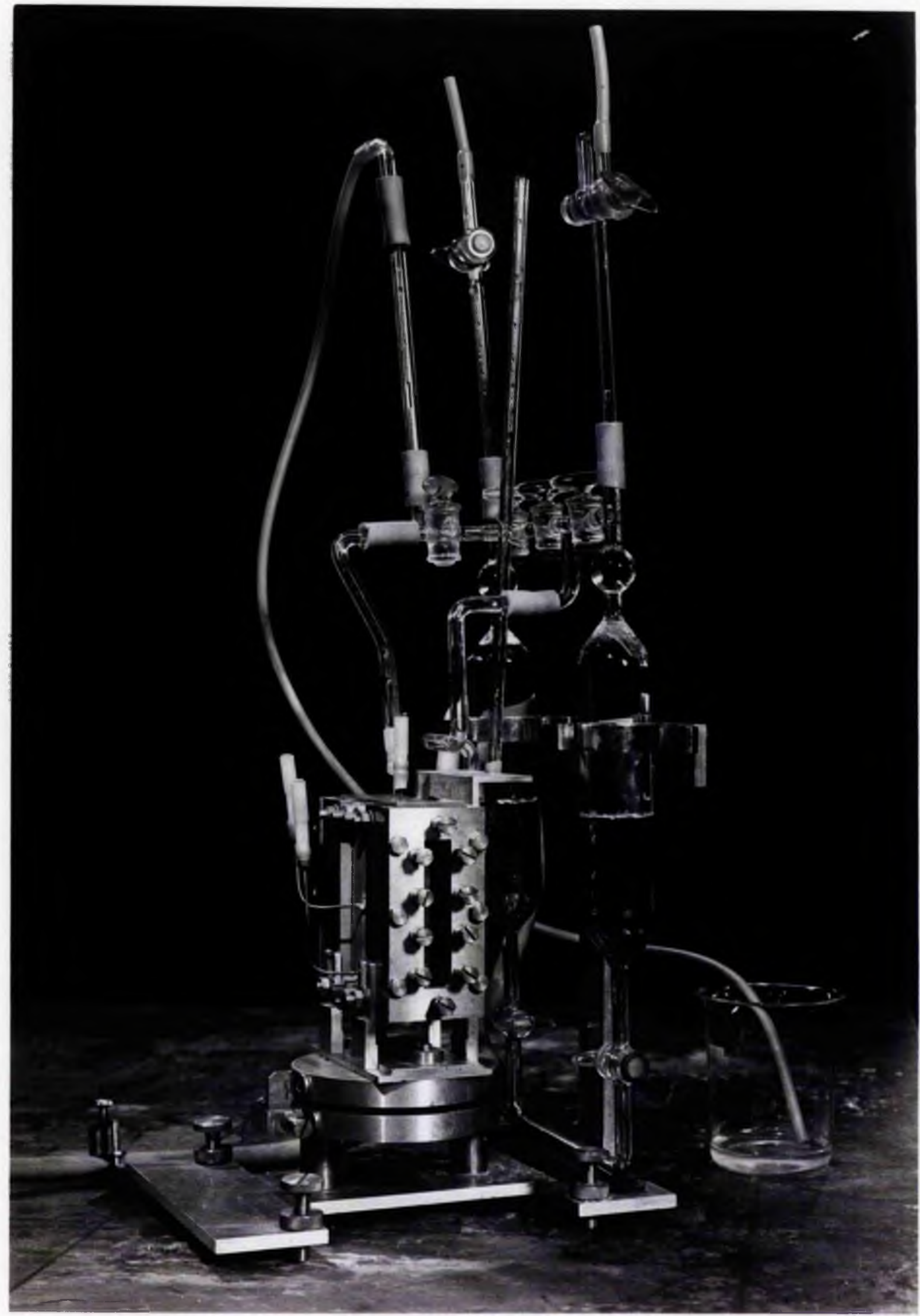

Fig.10.

The Flowing-Junction Cell. 


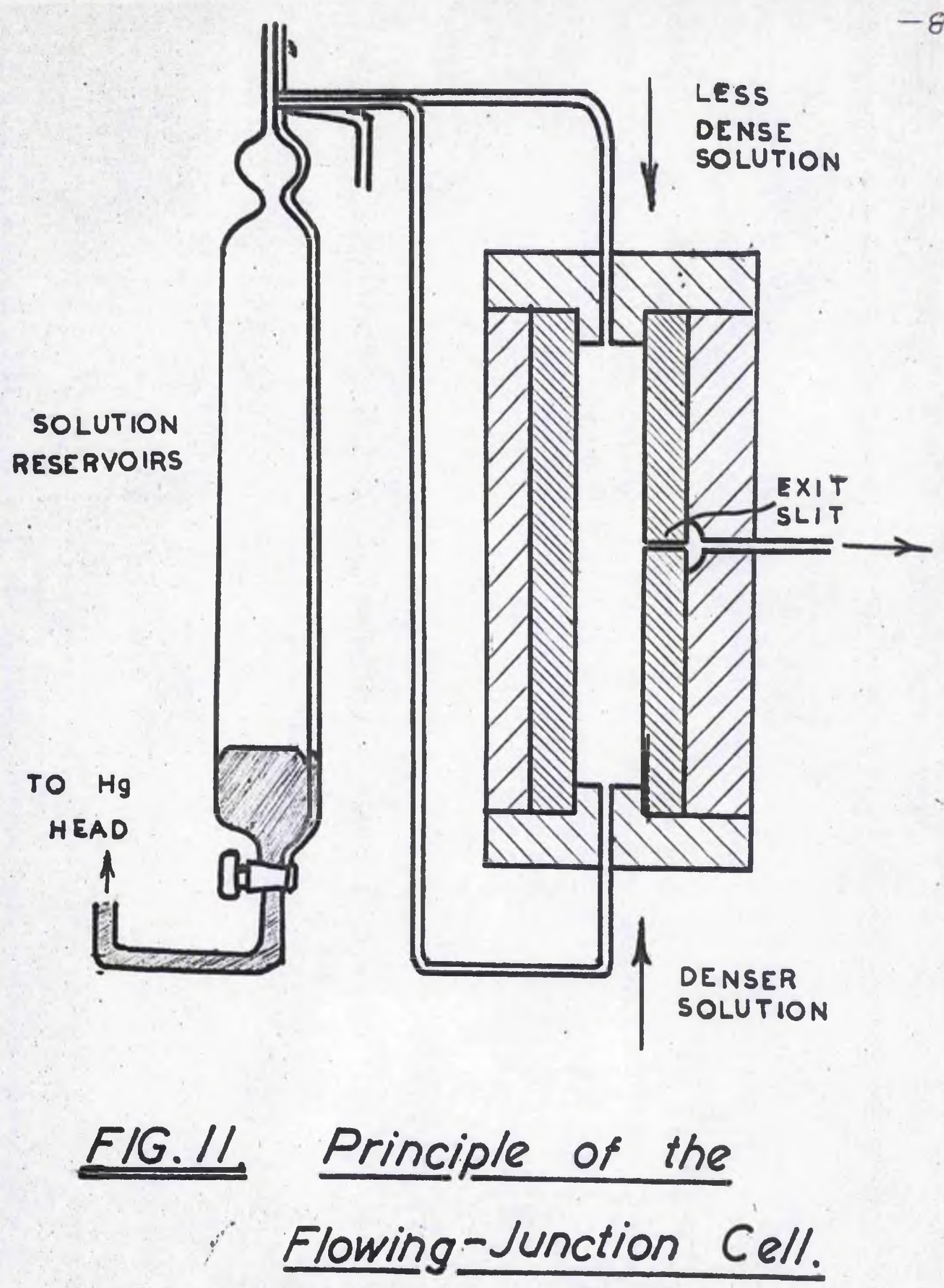


Fig.11. For clarity, the cell has been show with one chambor onlys though in actual fact it has two chambers. The second chamber was originaliy intended as a reference chamber (for Rayleigh fringes), but now serves as a reserve. The cel]. is constructed throughout in stainless steel. Sections are bolted together to form the two chambers, and the ends are ground plane and parallel. The optical path length through the cell is $63 \mathrm{~mm}$. Optical glass rlats ( $\frac{3}{8} "$ thick) are pressed into contact with the cell ends by ten spring-held pressure screws. A polythone gasket smeared with silicone grease separates the glass flats from the steel faces and copper and rubbor gaskets distribute tho screw pressure on the glass flats.

In the centre of the cell, running horizontally, is the

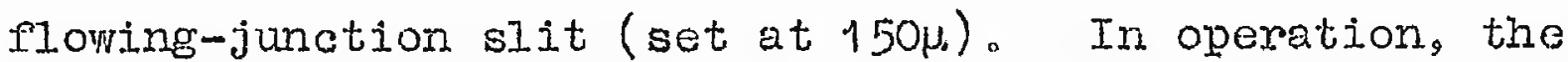
two solutions of differing density meet and flow out of this siit, the exit being a capillary tube.

The solutions are contained in two reservoirs, which are connected to the cell by a manifold with suitable taps. A mercury reservoir, of variable height, provides the head mecessary for the solution and solvent to flow into the cell. 
III (e) Experimenta1 Technique

The cell is cleaned and flushed with distilled water and air, before being finally flushed with solvent, the liguid reservoirs are cleaned, flushed, and filled with solvent and solution. The solution strength found to be most convenient to work with in the interferometer was about 0.06 weight $\%$ difference. (Hence, as an example, for determining the diffusivity in a very dilute solution, one reservoir will contain pure solvent and the other will contain a solution of $0.6 \mathrm{~g} / \mathrm{litre}$. For the diffusivity in, say, a $100 \mathrm{~g} /$ Iitre solution, one reservoir is filled with $100 \mathrm{~g} /$ litre solution and the other with $100.6 \mathrm{~g} /$ litre solutiono)

When the coll and reservoirs have been filled up, the thermostat for the room is set at $25^{\circ} \mathrm{C}$. The cell is then left set up on the optical bench for about $12-14$ hours (i.e.overnight)。 This will ensure thermal equilibrium between the two solutions. The establishment of thermal equilibrium is most important, for it must be appreciated that small differences in temperature or slight mechanical vibracions transmitted to the cell can cause much more rapid mixing of the diffusing substances than the pure diffusion process itself. A temperature dirference of $0.1^{\circ} \mathrm{C}$ between solutions is quite sufficient to completely aiter the shape of the concentration - distribution curve 


\section{$-840$}

as recorded by this interferometer.

Once thermal equilibrium has been established, the solution (or denser solution) is admitted to the bottom of the cell. The solvent (or less dense solution) is admitted to the top of the cell. The mercury reservoir is used for the necessary head to provide the flow of solutions, and a fine control tap on tho mercury inlet adjusts the rate of Plow. When the solutions are admitted to the cell, the sidestream (from the flowing-junction slit) is started. By carciul control of the Iiquid flowrates the two solutions are caused to meot and flow out at the slit, forming a sharp interrace.

A very careful control of the flowrate is necessary to obtain a sharp interface. Practical experience in operating the cell determines this rate, for it varies with the viscosity of the solutions being used. As a rough guide, a flowrate out of the exit sitt of about 20-40 arops a minute is nceded.

Once the interface is formed, it is left for about 20 minutes to sharpen and establish itself. In the meantime the mercury vapour lamp has been switched on (about 20 minutes is needed before maximum intensity is reached) and the camera magazine is loaded.

Visual inspection of the interface is made by examining the image on a ground-glass screen (with magnifying lens 
$-85=$ 


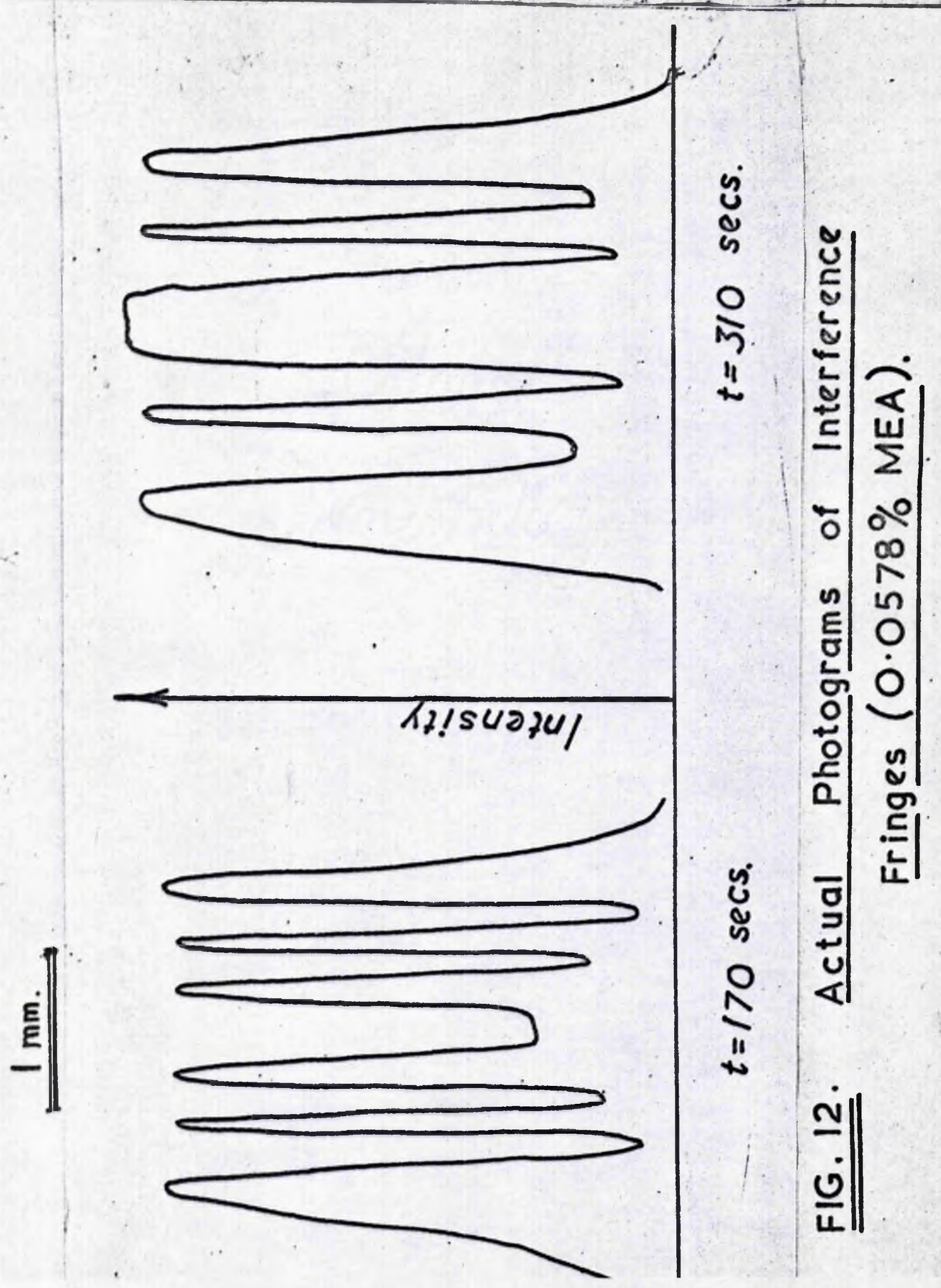



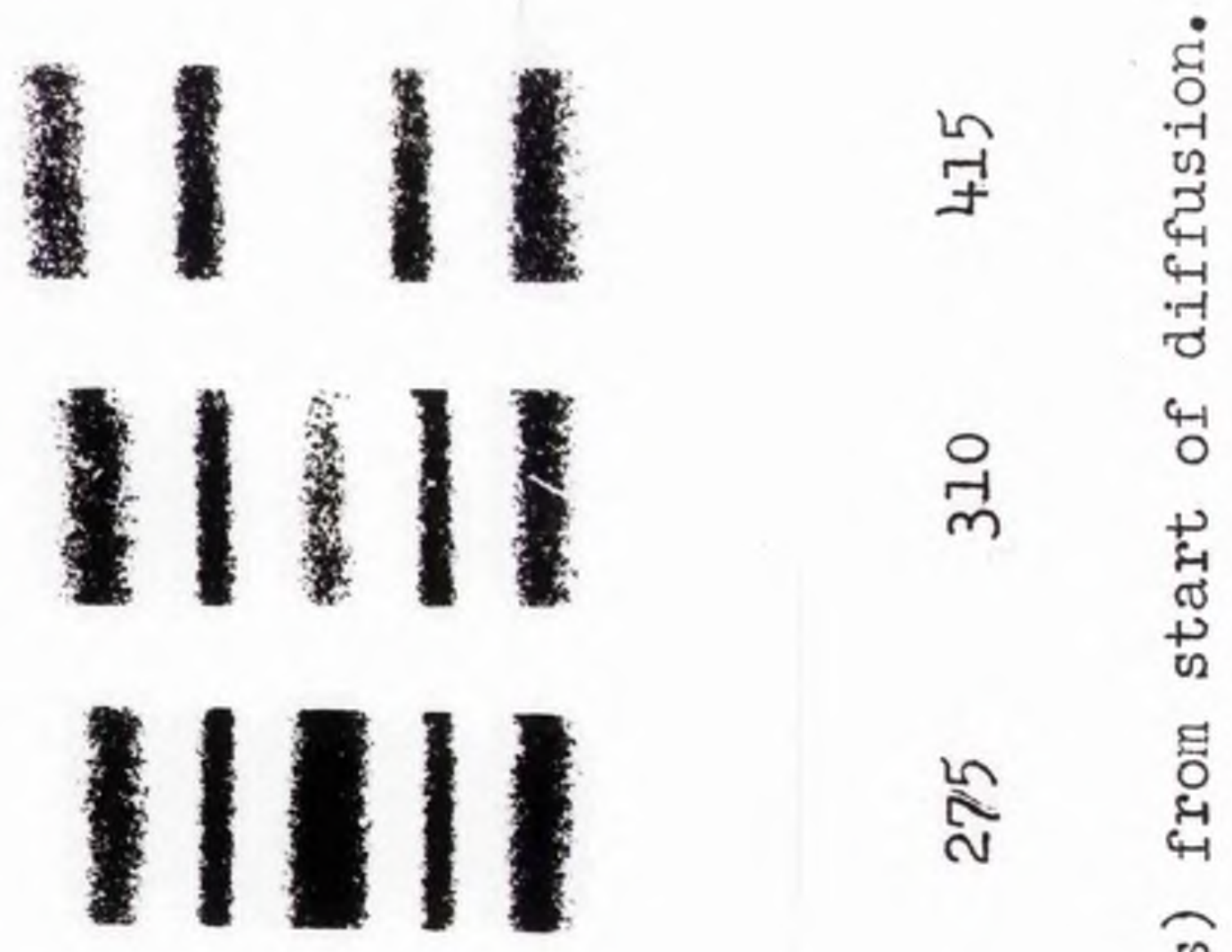

ลิ
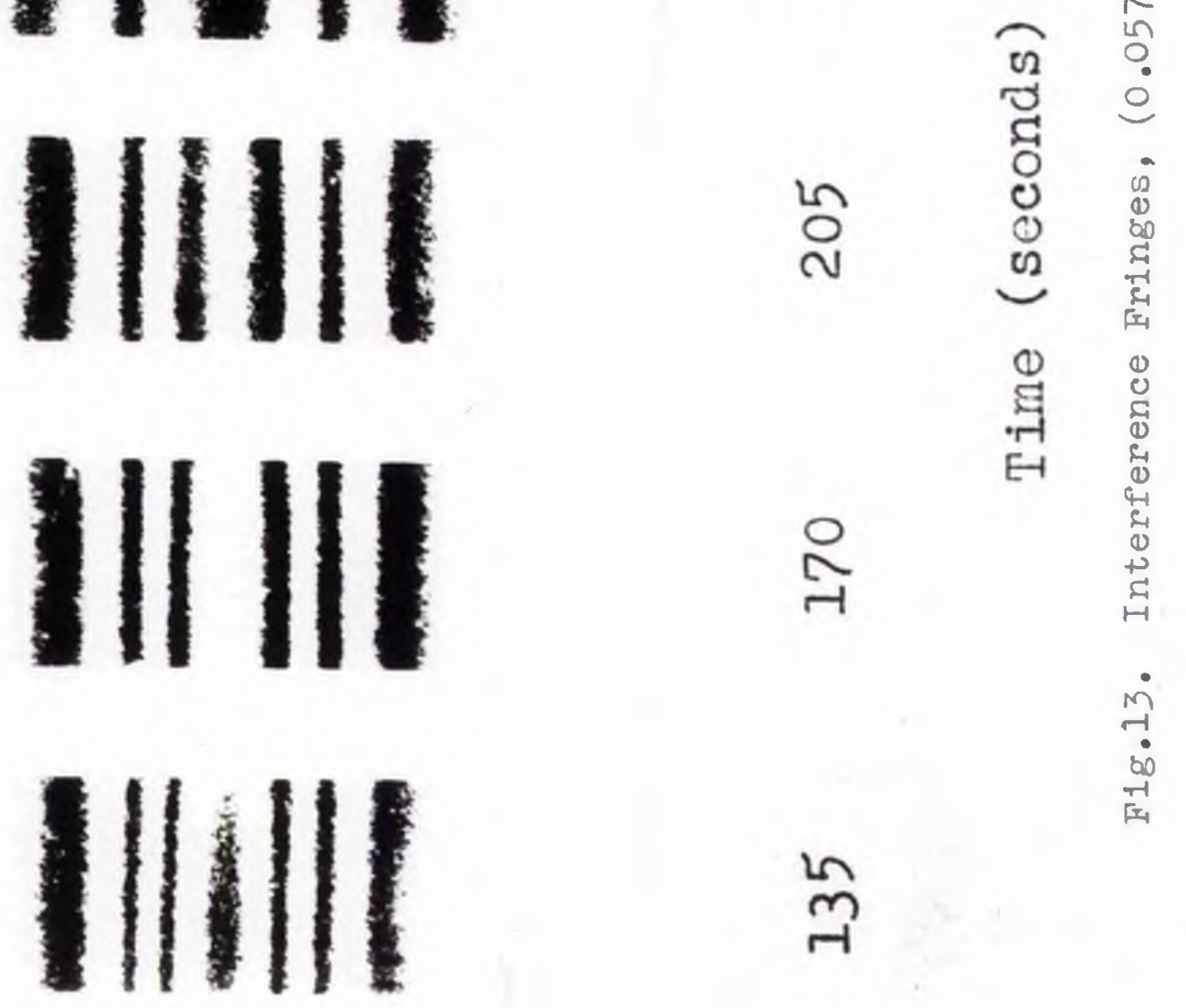


$$
-8700
$$

attached).

Before starting the recording of the diffusion process, the camera is placed in position (the cell image is projected directly on to the film). The time control unit is set for 5 second exposures at 30 second intervals. As soon as the first exposure is taken (of the interface) the relevant taps on the cell are shut, thus stopping the sidestream discharge and startiug the diffusion process in the $\operatorname{cell}$.

Photographic recording of the diffusion process is continued until inspection of the image reveals that the next-to-outermost fringe pair has merged. With a $0.06 \%$ concentration difference, this will occur after about 60 exposures (depending on the diffusion coefficient).

The movement of the fringe-pairs as recorded photom graphically is measured primarily by a Joyce-Loebl double. beam recording microdensitometer. This instrument translates the intensity variations on the film (due to the fringes) into graphical representations (photograms)。

A typical series of fringe pairs is shown in Fig.13, and associated photograms are shown in Fig.12.

The distance between fringe peaks is readily determined from the photograms, and hence $(2 x)$ for any one fringe pair is measured. (The accuracy of the measurement is about \pm $0.002 \mathrm{~mm}$ overall). 
Where the microdensitometer is limited in resolution (as, for example, when a number of fringe-pairs are very closely spaced together), a cathetometer is used to measure $(2 x)$, (to an accuracy of $\pm 0.005 \mathrm{~mm}$ )。 From these measurements, therefore, a recording of the experimental values of $(2 x)^{2}$ against $t$ may be made, and the diffusion coefficient evaluated by using one of the calculation methods described. 
IV. FEXPERTMEINTAI RESUITS。

IV (a).Example of the Calculation of Diffusivity.

An example of the calculation of diffusivity from the fringe movement racorded by the wavefront shearing: interferometer for diethanolamine-water is illustrated below.

Run No. DEA 8 ,

Temperature

Concentration of DEA solutions.

Concentration difference between solutions :0.8 $\mathrm{g} / 1$ itre

Fringe pair observed

Magnification factor of interferometer
$: 25.3^{\circ} \mathrm{C}$

: $462.5 \mathrm{~g} /$ Iitre

: FP. 4

$: 1.2482$

(Thus, $2 x(m)$, - the fringe spacing measured on the film, must be divided by 1.2482 to obtain the true $2 \mathrm{x}$ ).

The fringempajx movement is tabulated in lable 1. With $\Delta t=0$, the best $D$ and $t_{i}$ to itt these experimental points are calculated. From this $D$ and $t_{1}$, $(2 x)_{i B}^{2}$ is calculated, and the regression curve of $(2 x)^{2}$ on $t$ is drawn in comparison with the expeximental points ( Fig, 15) . The standard exror of estimate between the tiwo 
curves, s , is calculated big equation 93.

$\Delta t$ is then increased by repeated 10 second increments, and the besturititing $D$ and $t_{1}$ are recorded, together with the calculated $(2 x)_{i B}^{2}$ and $s_{0}$ For the diffusion of $462.5 \mathrm{~g} /$ Iitre DEA - water, these figures are given in table 2. The calculation is illustrated graphically in Fig. 14 .

From the plot of st against $\Delta t$, the curve passes through a minimum at $\Lambda t=89 \operatorname{secs}($ the possible error in estimating $\Delta t$ here is about \pm 2 secs. $\Delta t$ can, of course, be determined extremely accurately if necessaxy, by altering the $\Delta t$ increments in the computer programme)。

A curve drawn through the experimental points gives a $(2 x)_{\text {iA }}^{2}$ value of 2.074 mmo The values of $(2 x)_{i B}^{2}$ at each $\Delta t$ are compared with this value, and at $\Delta t=89$ secs $(2 x)_{i A}^{2}=(2 x)_{i B}^{2}$

Thus $\triangle t$ is calculated to be 89 secs. From this value of $\Delta t, D$ is found to be $3.184 \times 10^{-6} \mathrm{~cm}^{2} /$ iec. The regression curve of $(2 x)^{2}$ on $t$, for $D=3.18 .7$ $x 10^{-6}$ and $\Delta t=90$ secs $i s$ compared with the experimental points in Fig.16. It can be seen that there is good agreement; between the experimental points and this regression curve. 
Table 3

Fringe-Pair (F.4) movement recorded during Diffusion

of $462.5 \mathrm{~g} / \mathrm{lit}$ ire DEA-water.

\begin{tabular}{|c|c|c|c|c|c|}
\hline $\begin{array}{c}t \\
\text { secs }\end{array}$ & $\begin{array}{c}2 \times(m) \\
\mathrm{mm}\end{array}$ & $\begin{array}{c}(2 x)^{2} \\
\mathrm{~mm}^{2}\end{array}$ & $\begin{array}{c}t \\
s \ominus c s\end{array}$ & $\begin{array}{c}2 \mathrm{x}(\mathrm{m}) \\
\mathrm{mm} .\end{array}$ & $\begin{array}{c}(2 \mathrm{x})^{2} \\
\min _{\mathrm{ia}}^{2}\end{array}$ \\
\hline 170 & L.398: & 1.2544 & 905 & 1.7773 & 2.0177 \\
\hline 240 & 1.580 & 1.6023 & 940 & 1.758 & 1.9837 \\
\hline 275 & 1.633 & 1.7126 & 975 & 1.740 & 1.9433 \\
\hline 310 & 1.665 & 1.7793 & 1010 & 1.740 & 1.9433 \\
\hline 345 & 1.690 & 1.8332 & 1045 & 1.720 & 1.8988 \\
\hline 380 & 1.720 & 1.8988 & 1080 & 1.715 & 1.8878 \\
\hline 4.75 & 1.733 & $1.927 \%$ & 1715 & 1.690 & 1.8332 \\
\hline 450 & 1.765 & 1.9995 & 1750 & 1.670 & 2.7900 \\
\hline $48: 5$ & 1.7755 & $I_{.} .9769$ & 1185 & 1.650 & 1.7474 \\
\hline 520 & 1.780 & 2.0336 & 1220 & 1.645 & 1.7369 \\
\hline 555 & 1.790 & 2.0565 & 2255 & 1.620 & 1.6845 \\
\hline 590 & 1.795 & 2.0680 & 1290 & 1.605 & 1.6534 \\
\hline 625 & 1.795 & 2.0680 & 1325 & 1.585 & 1.6 .125 \\
\hline 660 & 1.793 & 2.0634 & 1360 & 1.560 & 1.5620 \\
\hline 695 & 1.795 & 2.0680 & 1395 & 1.545 & 1.5321 \\
\hline 730 & 1.798 & 2.0750 & 1430 & 1.520 & 1.4829 \\
\hline 800 & 1.7900 & 2.0565 & 1465 & 1.483 & 1.41 .16 \\
\hline 835 & 1.788 & 2.0519 & 1500 & 1.455 & 1.3588 \\
\hline 870 & 1.780 & 2.0336 & 1535 & 1.430 & 1.3125 \\
\hline
\end{tabular}


Table 2.

Calculation to determine $\mathrm{D}$ and $t_{i}(462.5 \mathrm{~g} /$ litre DEA-water $)$.

\begin{tabular}{|c|c|c|c|c|}
\hline \multirow[t]{2}{*}{$\Delta t$} & \multicolumn{3}{|c|}{$\begin{array}{l}\text { Calculated by method of least } \\
\text { Squares to best fit IxptI. points }\end{array}$} & \multirow{2}{*}{$\begin{array}{l}\text { Standard } \\
\text { Error of } \\
\text { Estumate } \\
\qquad s\left(\times 1: 0^{2}\right)\end{array}$} \\
\hline & $\mathrm{cm}^{2} / \mathrm{sec} \times 10^{6}$ & $t_{i}$ & $(2 x)_{i B}^{2}$ & \\
\hline 0 & 3.5443 & 747.77 & 2.1203 & 6.955 \\
\hline 10 & 3.4994 & 755.27 & $2.114+3$ & 6.293 \\
\hline 20 & 3.4559 & 762.73 & 2.2 .087 & 5.696 \\
\hline 30 & 3.413 .4 & 770.21 & 2.1032 & 5.169 \\
\hline 40 & $3 \cdot 3722$ & 777.66 & 2.0979 & 4.715 \\
\hline 50 & $3 \cdot 3316$ & 785.16 & 2.0927 & 4.34 .1 \\
\hline 60 & 3.2926 & 792.59 & 2.0877 & 4.053 \\
\hline 70 & 3.2542 & 800.06 & 2.0829 & 3.854 \\
\hline 80 & 3.2167 & $807: 54$ & 2.0781 & 3.746 \\
\hline 90 & 3.1806 & 814.96 & 2.0737 & 36724 \\
\hline 100 & 3.1451 & 822.42 & 2.0693 & 3.779 \\
\hline 110 & 3.3 .208 & 829.80 & 2.0651 & 3.899 \\
\hline 120 & 3.0770 & 837.24 & 2.0609 & 4.070 \\
\hline 130 & 3.0443 & 844.62 & 2.0570 & 4.281 \\
\hline
\end{tabular}




\section{FIG. 14}
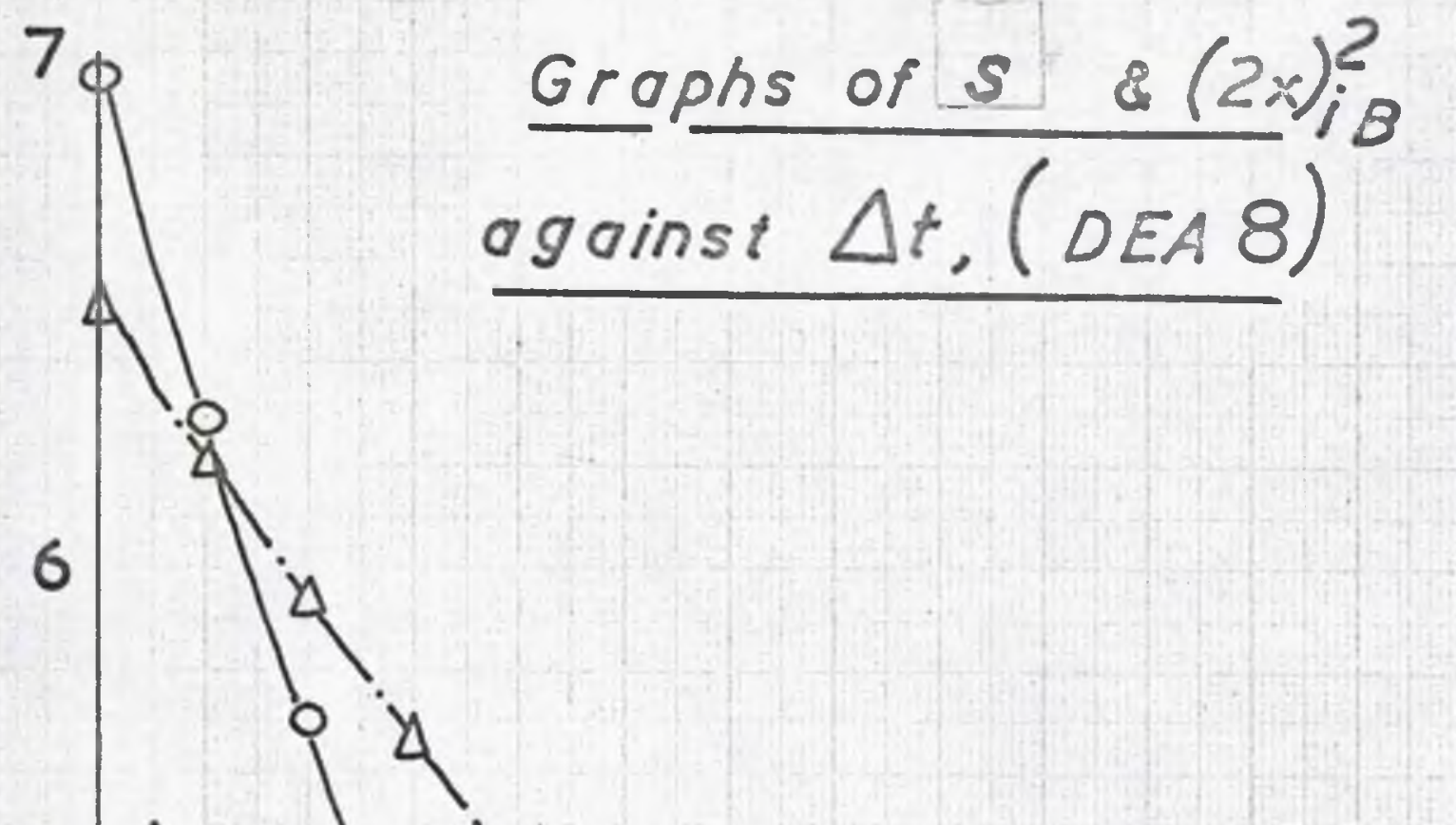


$$
-94-
$$




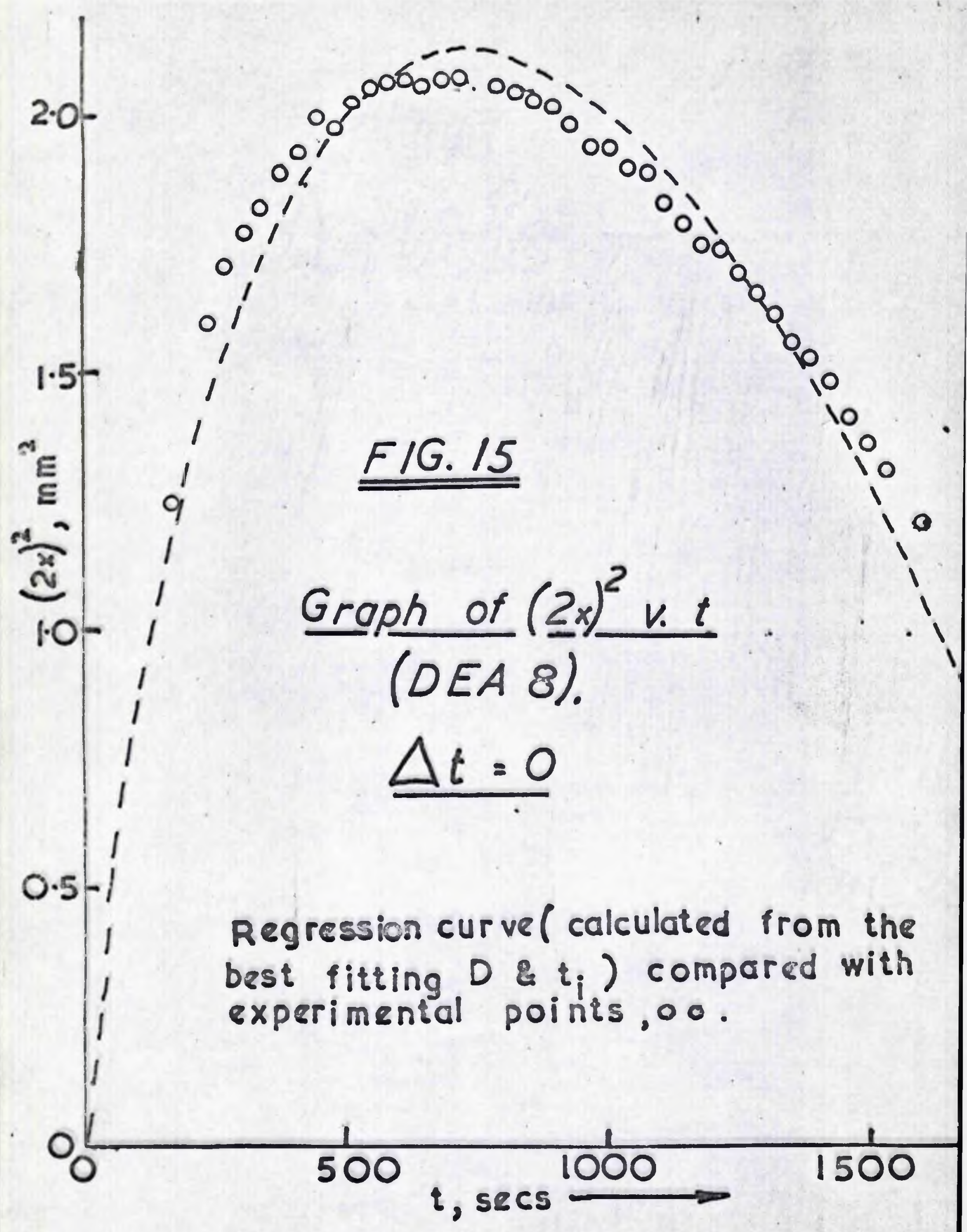




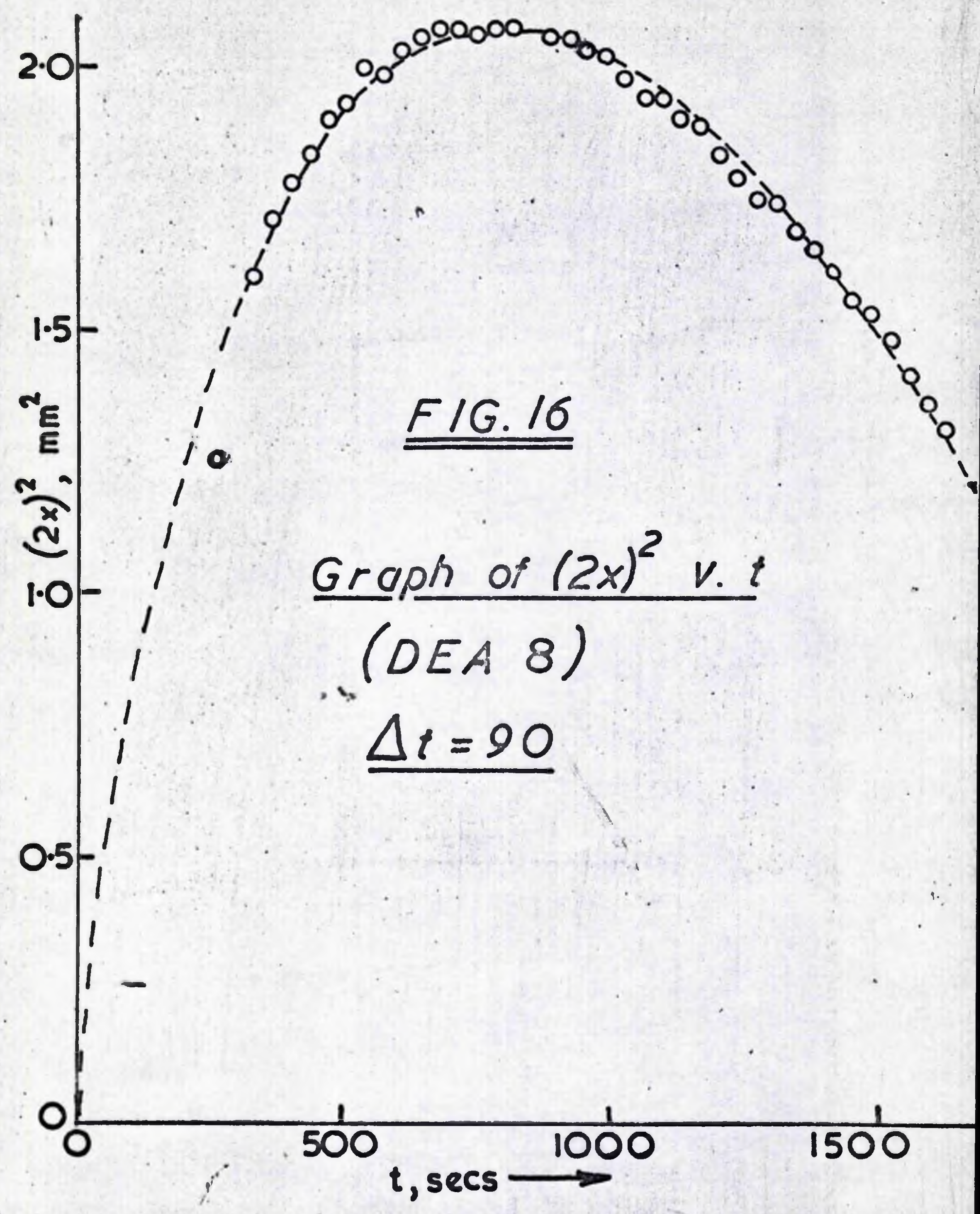


The accuracy of the calculation is detiomined by considering the estimation of $\Delta t$ ( 2 secs). The variation in $D$ ( calculated by least squares curvemfitting) per sec. $(\Delta t)=0.0036 \times 10^{-6} \cdot \mathrm{cm}^{2} / \mathrm{sec}$.

$\therefore$ Accuracy of determination of $D= \pm 0.007 \times 10^{0.6} \mathrm{~cm}^{2} / \mathrm{sec}$ $=0.2 \%$

IV (b). Diffusional Measurements for the Systems Sucrose-Water, and Ethanolaminesm Water.

Diffusion coefficients were measured for four dilute sucrose-water solutions as. a preliminary calibration of the interferometer. The following values for the diffusion coefficient of very dilute sucrosewwer solutions were obtained ( at $\left.25^{\circ} \mathrm{C}\right) ;-$

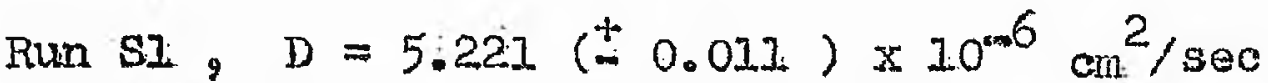

$$
\begin{aligned}
& \text { S2 }, D=5.205( \pm 0.015) \text { " } " \text { " } \\
& \text { S3, } D=5.216( \pm 0.012) \text { " " " }
\end{aligned}
$$

In run 53 , a low value of $\mathrm{D}\left(=5.13 \times 10^{-6}\right)$ wa.s obtained, with only limited accuracy. This was almost certainly due 
to an electrical failure, which interfered with the thermostatide contirol of the experiment, However, the values obtained in rung SI, $Z_{2}$ and 4 agree very well with the accepted values for sucrosemwater as obtrained by previous workers $(28)(60)(61)$.

Diffusion coefficierts for the syrstems monomethanol-amine/water, diethanolamine/water, and triethanolamine/waitex were measured over a range of concentrations.

A. summaxy of these measumements is given in table 3 . Detalized fringe measurements axe given in section (c).

The diffusivitionconcentration curves por the amines, as measured, are shown in Fig. $17 \%$ 
$-98-$ 


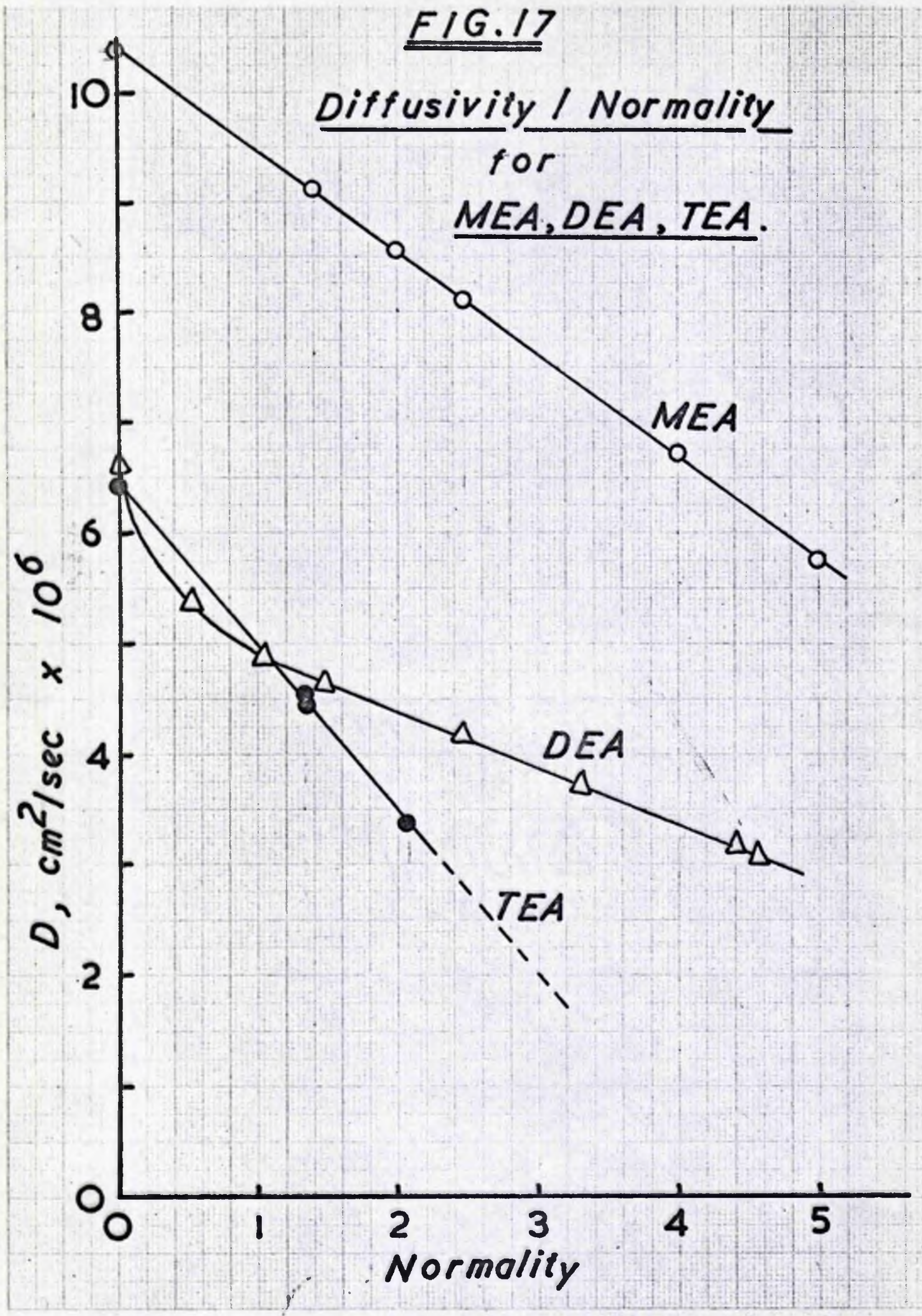




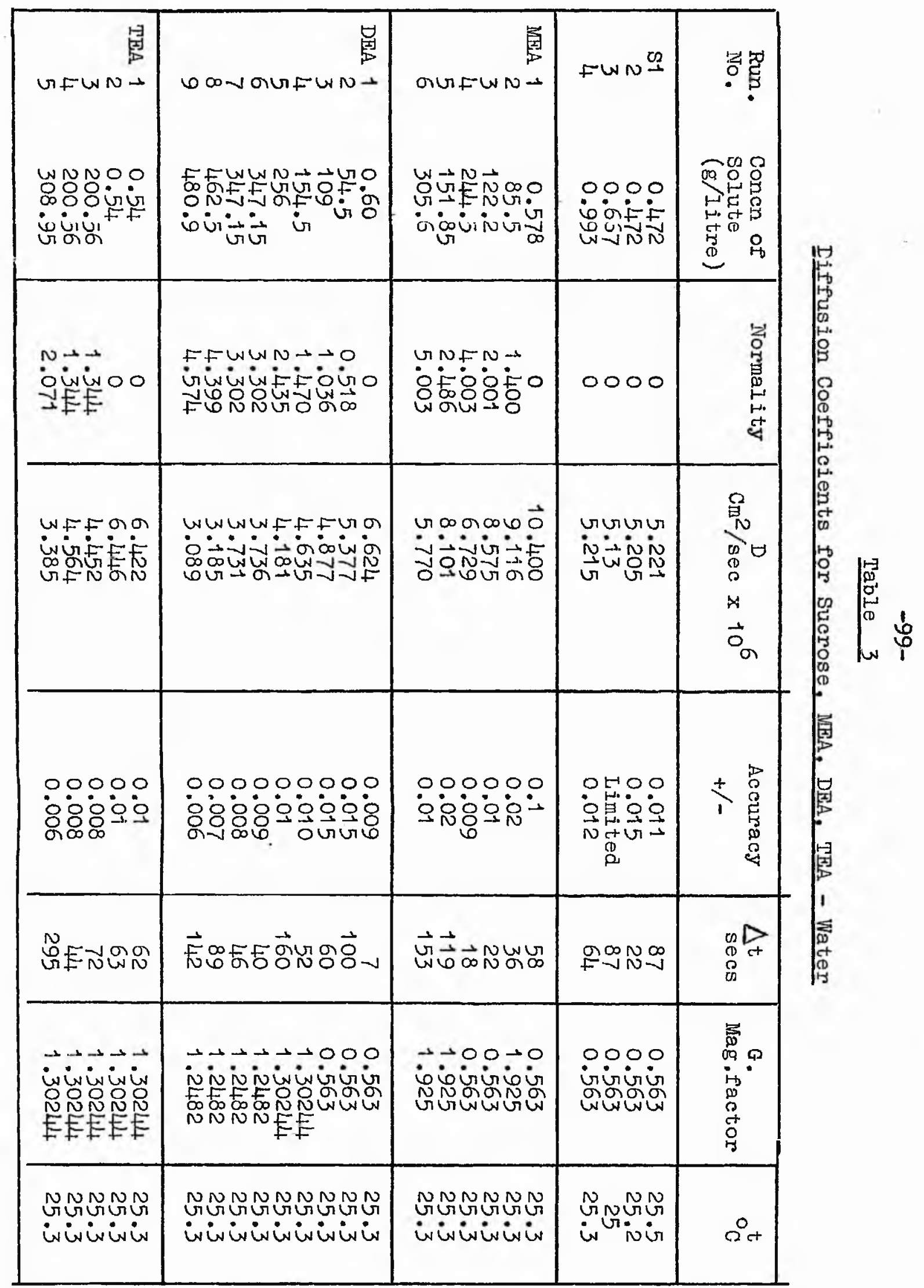


IV(c). Tabulated Resultiso

$\begin{array}{lc}\text { Run No.s } & \text { SIL to } 54 \\ & \text { MFAI to MEA6 } \\ & \text { DEAI to DEA9 } \\ & \text { TEAI to TEAS }\end{array}$

The resulits are tabulated in the following fommon

ii) Concentration of diffusing solution.

2) Temperature.

3) Fringemair observed (outermost $=I_{1}$ )。

4) Tabulated measurements of fringe-pair movement.

a) time of exposure (film frame).

b) Measured value of $2 \times$ (on the film).

c) Actual value or $(2 x)^{2}$.

5) Magnification factor, G.

6) Diffusion Coefficient calculatied, D.

7) Accuracy of measurement of $D$.

8) Zelro-time correction factor, $\triangle t$. 
O. 0472 WT ${ }_{0} P C T{ }_{0}$ SUCROSE WATER

$$
\text { t. } \frac{2505 . D E G_{B} C}{\ldots .}
$$

FoP:2

\begin{tabular}{|c|c|c|c|}
\hline$T$ & $2 \times(M)$ & & $a X S Q D$ \\
\hline (SECS) & $(\mathrm{MM})$ & & $(M H)_{2}$ \\
\hline 99 & $0 \circ 72^{3}$ & & $I_{0}, \overline{8}_{4}$ \\
\hline 33 & 0.740 & & $\operatorname{I07293}$ \\
\hline$x 65$ & $0.77 x$ & & $1_{0} 8769$ \\
\hline$x 9^{8}$ & $0.78 \mathrm{I}$ & & $x \cdot 923^{8}$ \\
\hline $23^{\mathrm{T}}$ & 0.799 & & $200 \div 64$ \\
\hline 264 & 0.825 & & $20 x 25^{8}$ \\
\hline$\quad 297$ & $0.82 \mathrm{~L}$ & & $20 \pm 258$ \\
\hline$\therefore 33^{\circ}$ & 0.835 & & 201993 \\
\hline .363 & 0.839 & & $20223^{I}$ \\
\hline $\begin{array}{r}396 \\
439\end{array}$ & $\begin{array}{l}0.850 \\
0.850\end{array}$ & & 202001 \\
\hline 462 & 0.856 & & $283 \mathrm{xO}_{4}$ \\
\hline 495 & 0.856 & & 2033.04 \\
\hline $52^{8}$ & 0.850 & & $30280 x$ \\
\hline 562 & 0.839 & & $20223 x$ \\
\hline 594 & 0.833. & & $20 \pi 904$ \\
\hline 627 & $0.8 \pm 6$ & & 201025 \\
\hline 660 & 0.806 & & 200506 \\
\hline $693^{\circ}$ & 0.790 & & $\pi \cdot 96^{8} 4$ \\
\hline 726 & $007^{8} 5$ & & $x \cdot 9460$ \\
\hline 759 & 0.766 & • & Io 8496 \\
\hline 792 & 0.744 & & $x \circ 7450$ \\
\hline 825 & 0.733 & & $\therefore 6900$ \\
\hline $85^{8}$ & $0.7^{2} 4$ & & $x_{0} 607^{8}$ \\
\hline $89 x$ & 0.676 & & $\pi .4400$ \\
\hline 924 & $0.65 \mathrm{x}$ & & xb3363 \\
\hline & $\cdots$ & & $\therefore$. \\
\hline
\end{tabular}

CALCULATEDOO $000^{\circ}$

$\stackrel{G}{D}(\mathrm{CH} 2 / \operatorname{SEC} \times 20 \mathrm{kn} 6)$ 0.56300 ACCURAGY $Y_{0}=+\%$ $5 \circ 22 x=-$ DELTA T (SECS) $0 \ldots 02 \pi$ 87 . 
$4|c| \ldots$ 
RUN $N O_{0} 5 / 3$

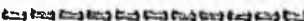

O047a WTOPCTO SUCROSE WATER

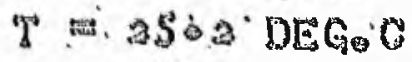

$$
\text { Fopo: }
$$

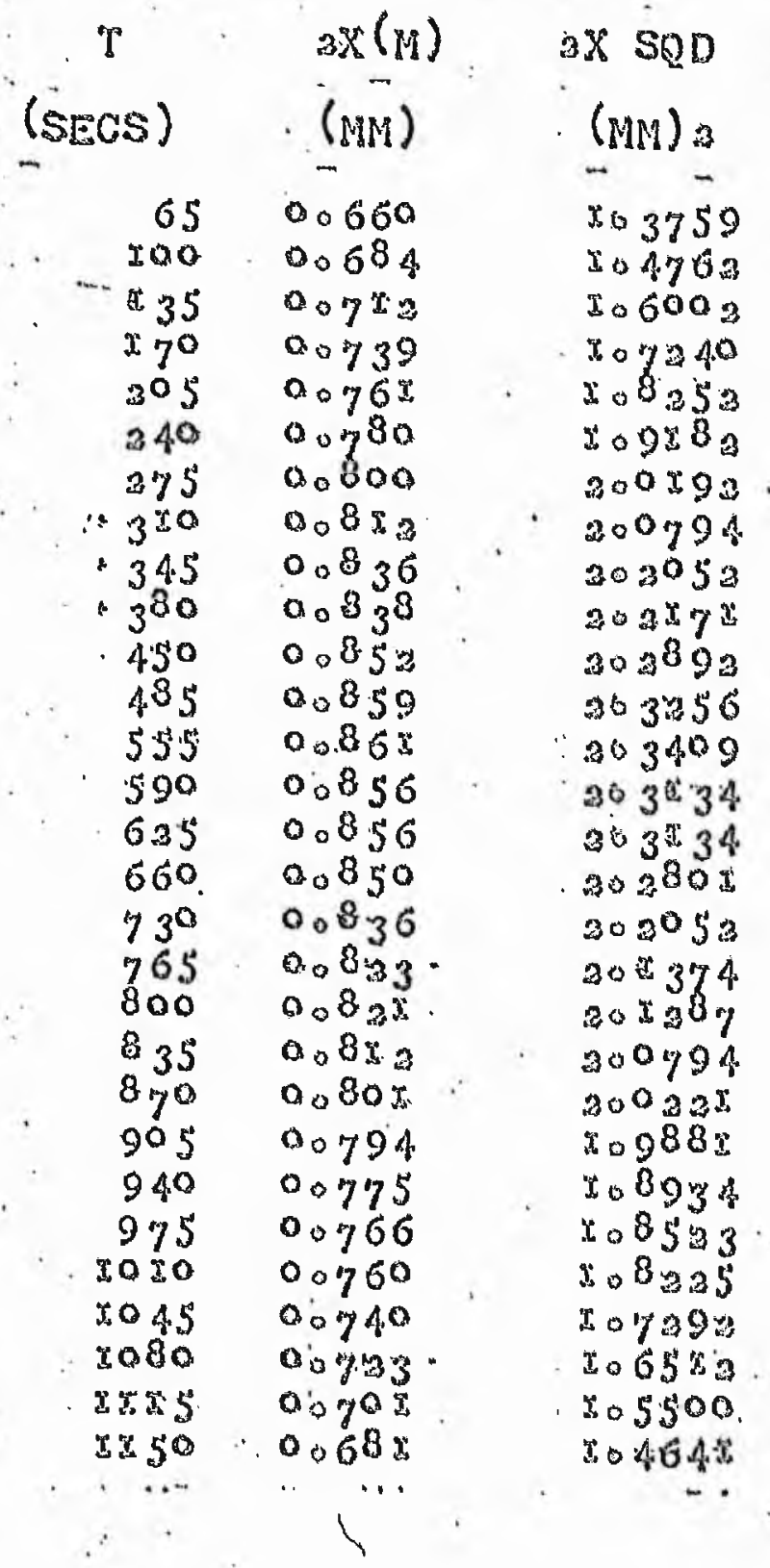

G. $(\mathrm{CM} 2 / \mathrm{SEC} \times 20 \mathrm{~m} 6)^{\text {tis }}$

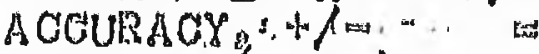
DELTA + $\%$ (SECS ) ins

$0.563^{\circ 00}$

50405

0005 
RUN NO. S/3

0.0667 WT.PCT. SUCROSE WATER

FP 2

$T$

$2 \times(i 1)$

2X SQD

(SECS)

(Min)

IOO

0.827

I 35

I $7^{\circ}$

205

240

3 10

- 345

- 380

415

$45^{\circ}$

$4^{8} 5$

520

555

590

625

660

695

$73^{\circ}$

765

335

$87^{\circ}$

905

940

975

IOIO

I 195

$I_{3} 80$

I 575

I 760

0.853

0.430

$0.9 I_{3}$

0.935

0. 974

0.997

I.0I9

1.036

I.0 44

I.06I

(iii) 3

3. 1580

2.3952

$3.143^{\circ}$

2.6376

$2 \cdot 7556$

2. 9929

3. 329

- $3 \cdot 2761$

- $3 \cdot 3^{8} 56$

$3 \cdot 4410$

$3 \cdot 5533$

- $3 \cdot 6320$

- $3 \cdot 6864$

$3 \cdot 7249$

$3 \cdot 7^{8} 3^{0}$

$3 \cdot 7636$

3.8809

- 3.9402

$3 \cdot 9402$

- $3 \cdot 960 \mathrm{~J}$

- $3 \cdot 910_{2}$

$3.373^{\circ}$

3.8809

$3 \cdot 873^{\circ}$

$3.873^{\circ}$

$3 \cdot 7^{3} 3^{\circ}$

$3 \cdot 6 \pm 00$

$3 \cdot 4708$

$3 \cdot 907^{\circ}$

$2 \cdot 37^{I} 6$ 
0.0 .993 WT PCT. SUCROSE WATER

7. $25 \div 3 . \mathrm{DEG}_{0} \mathrm{C}$

FoPos

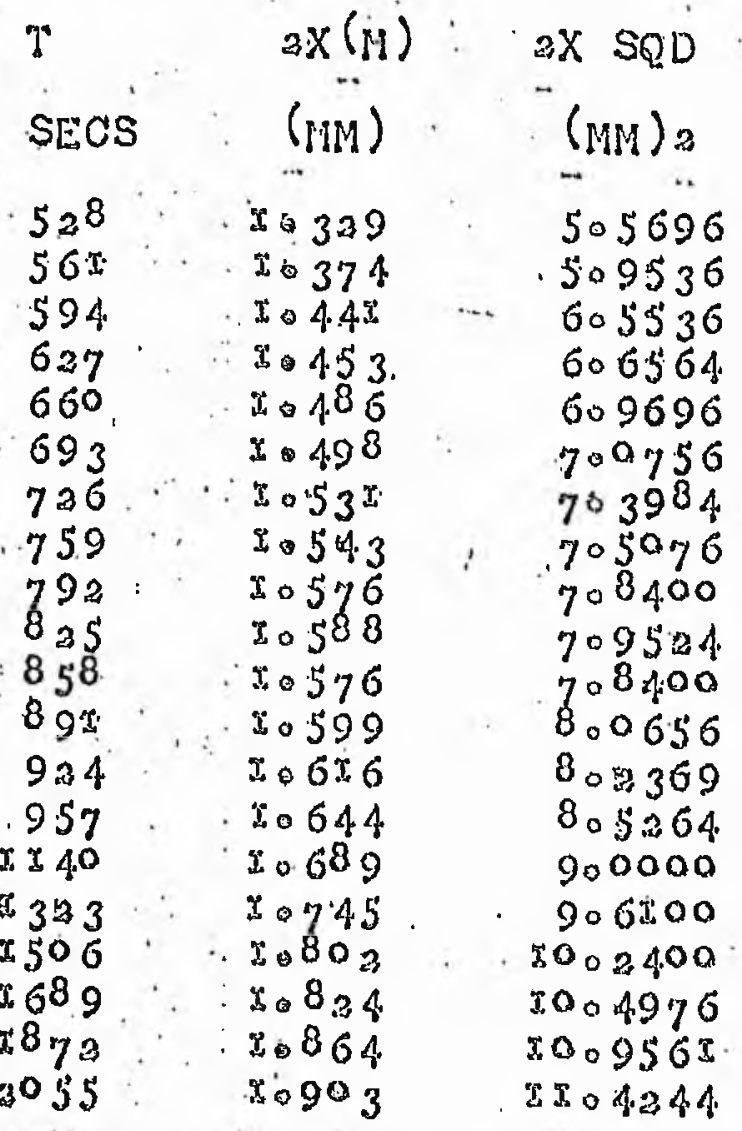

\begin{tabular}{|c|c|c|}
\hline $\mathrm{T}$ & $\operatorname{ax}(M)$ & $3 X$ SQD \\
\hline (SECS) & (MM) & $(M M)=$ \\
\hline $323^{8}$ & $x \circ 903$. & $\ddot{z} x \circ 4 \overrightarrow{2} 4.4$ \\
\hline 2425 & $x \circ 935$ & x 506064 \\
\hline 2605 & $x 0925$ & $x x_{0} 6964$ \\
\hline $27^{80}$ & $x 0925$ & $x 806964$ \\
\hline $297 \%$ & $x \in 909$ & $x x \circ 4925$ \\
\hline 3554 & $50903^{\circ}$ & 2004244 \\
\hline 4337 & $x \in 875$ & 5200889 \\
\hline 3520 & 80875 & 2300889 \\
\hline 3783 & 20835 & 3006276 \\
\hline 3886 & 20802 & 3002400 \\
\hline 4069 & $x \cdot 7^{3} 5$ & 2000489 \\
\hline 4242 & $\log 88$ & 908596 \\
\hline 44.55 & 20725 & 908486 \\
\hline 4608 & $x \circ 695^{\circ}$ & 900601. \\
\hline $4.79 t$ & $0633^{\circ}$ & 804,000 \\
\hline 4974 & \pm 0646 & $80: 369$ \\
\hline $\begin{array}{l}5.57 \\
5340\end{array}$ & $\begin{array}{l}30543 \\
x \circ 486\end{array}$ & 705076 \\
\hline 553 & $x 044 \pi$ & \\
\hline & $x 03 \% 7$ & So 4756 \\
\hline
\end{tabular}

CALCULLATEDO
G $(\mathrm{CM} 3 / \mathrm{SEC} \times 100.6)^{\circ} 0056300$ ACCURACY + 4 \% DELITA 'i' (SECS) E 64 .




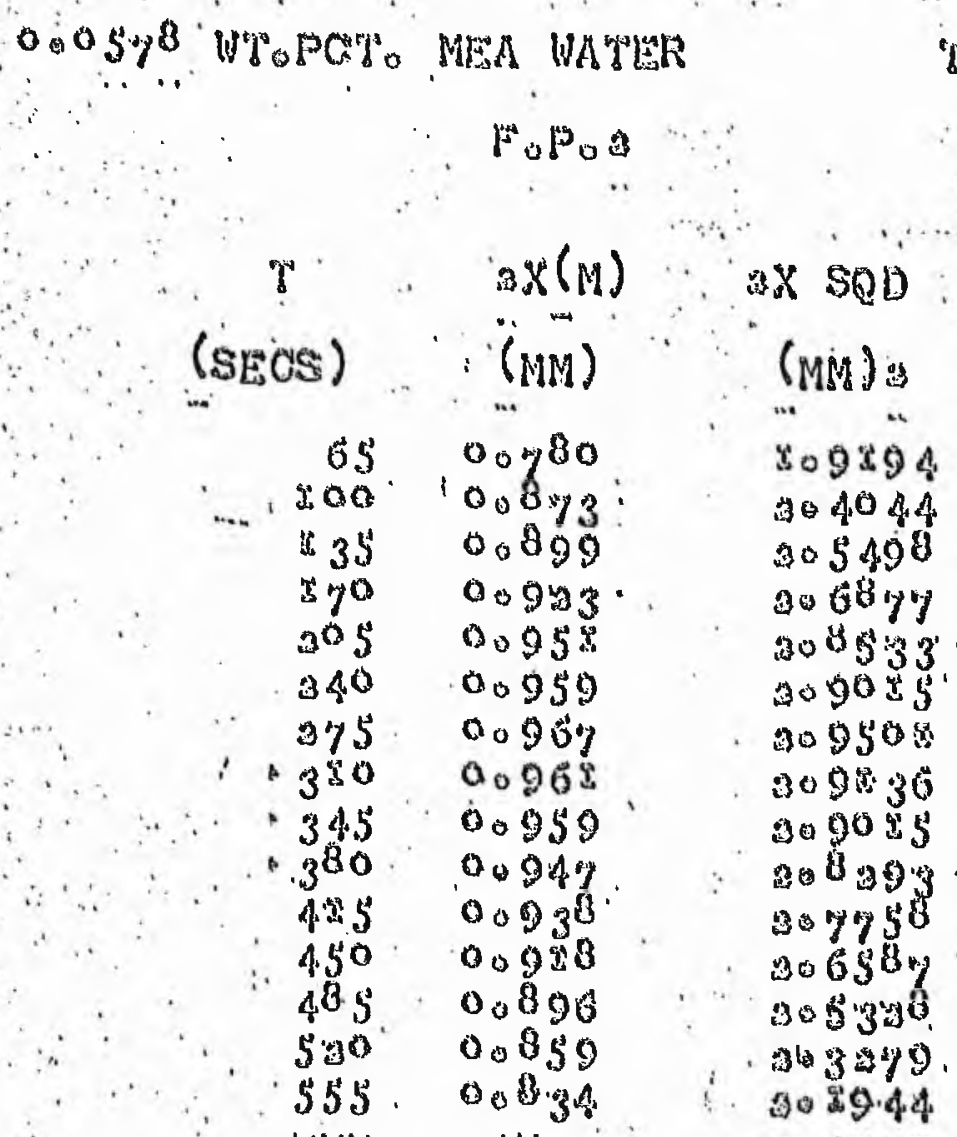

- $\quad 35-3-D E G$ 
RUN NOO MEA/A

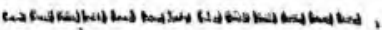

85.S Go/LITRE MEA WATER

$$
\text { Fopo }
$$

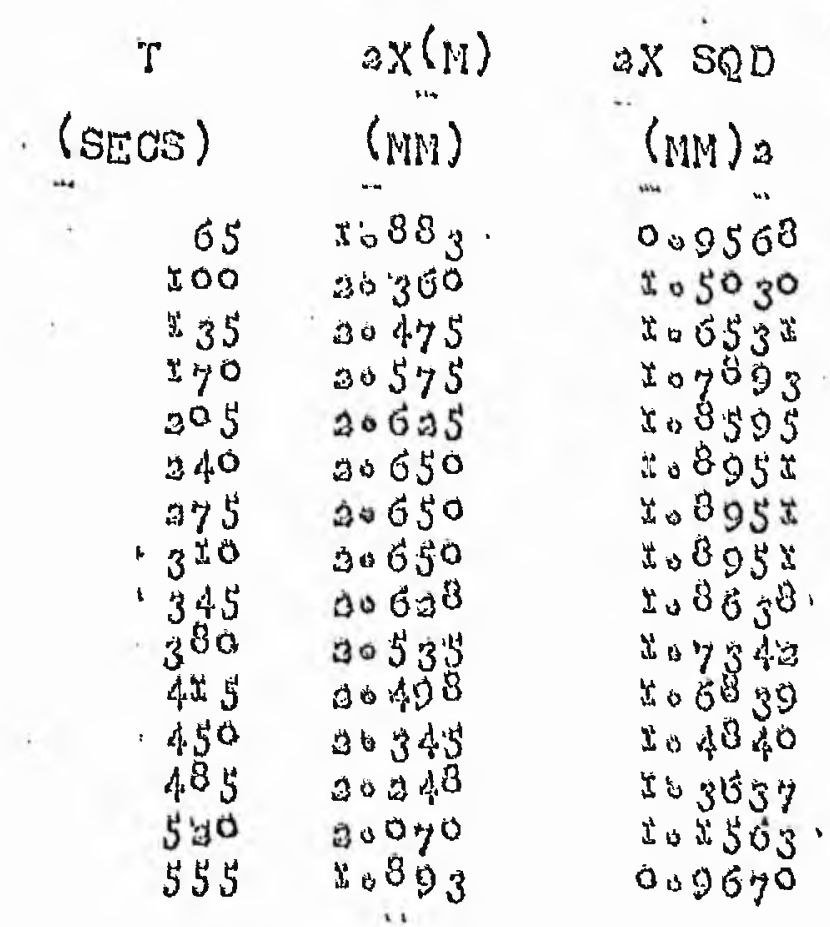

aX SQD

$-106-$

$$
T=25 \cdot 3 \cdot D E G \cdot C
$$

$(M M)_{2}$

0.9568

4.5030

प 6553

107093

5.8595

t艹 8954

4.8954

$4095 \mathrm{x}$

I. 3038

4 1340

10,80

I5 3057

Iक 2503 .

$0.967^{\circ}$

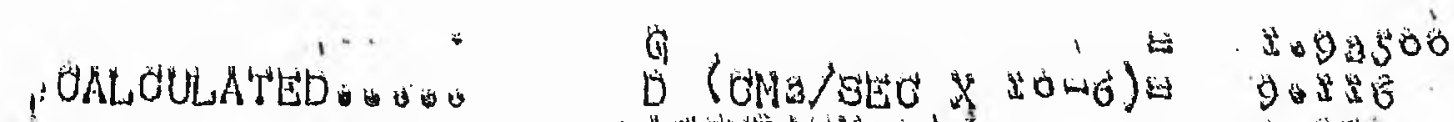

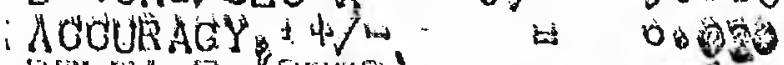


$I_{23.3} G_{\odot} / L I T R E$ MEA WATER

$$
\text { Fopoa }
$$

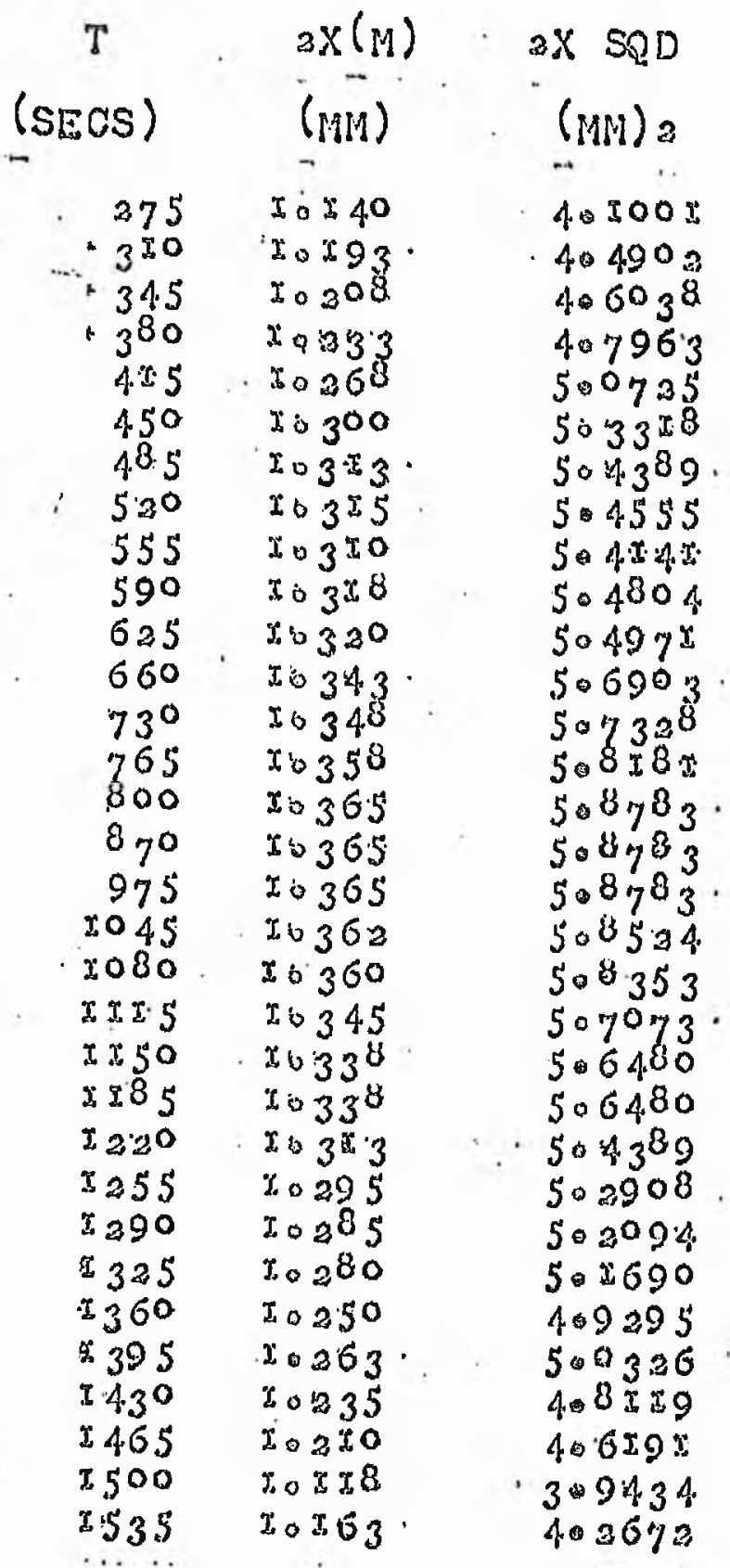

CAL CULATEDD.000

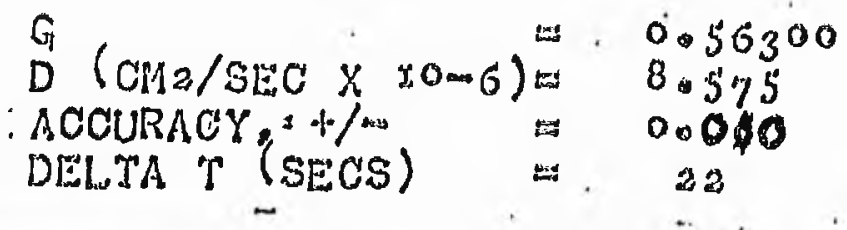


RUN $:$ OO. MEA/ts

$244 \cdot 5$ G./LITRE WEA HATER

$$
T=25 \cdot 3 \cdot D E G_{\cdot} \mathrm{C}
$$

$F \cdot P \cdot 3$

\begin{tabular}{|c|c|c|}
\hline$T$ & $2 \times(11)$ & $2 \%$ SQD \\
\hline Ecs) & $(\ldots i)$ & $(m)^{2}=$ \\
\hline 205 & $0.8 E=$ & $2 \cdot 3388$ \\
\hline$=\div 0$ & & $55 \div$ \\
\hline 275 & 0.900 & 2.5555 \\
\hline 3 I0 & 0.929 & $2 \cdot 7228$ \\
\hline 345 & $\begin{array}{l}0.929 \\
0.045\end{array}$ & $\begin{array}{l}2 \cdot 722^{3} \\
\therefore y^{3} 2^{2}\end{array}$ \\
\hline $\begin{array}{l}300 \\
415\end{array}$ & $\begin{array}{l}0.945 \\
0.960\end{array}$ & $\begin{array}{l}2.0174 \\
2.9075\end{array}$ \\
\hline 450 & 0.959 & $2.90 I 5$ \\
\hline $4^{85}$ & 0.954 & $2 \cdot \varepsilon_{7 I} I_{3}$ \\
\hline 520 & 0.960 & 2.9075 \\
\hline 555 & 0.960 & 2.9075 \\
\hline 590 & 0.258 & $2 \cdot 0$ \\
\hline 625 & 0.950 & 2.8473 \\
\hline 660 & 0.942 & 2.7995 \\
\hline $\begin{array}{l}765 \\
800\end{array}$ & $\begin{array}{l}0.912 \\
0.887\end{array}$ & $\begin{array}{l}2 \cdot 0241 \\
2 \cdot 4822\end{array}$ \\
\hline${ }^{8} 35$ & 0.885 & 2.4710 \\
\hline $87^{\circ}$ & $0.87 \%$ & $2 \cdot 4099$ \\
\hline 905 & & 2.3551 \\
\hline 940 & 0.347 & $2 \cdot 2533$ \\
\hline $\begin{array}{r}975 \\
\text { IOI0 }\end{array}$ & $\begin{array}{l}0.83 \\
0.805\end{array}$ & \\
\hline & & \\
\hline
\end{tabular}


351.85 Go/LITRE MEA WATER

$$
T=35 \cdot 3 \cdot D E G \cdot C
$$

$$
\text { FoP: } 3
$$

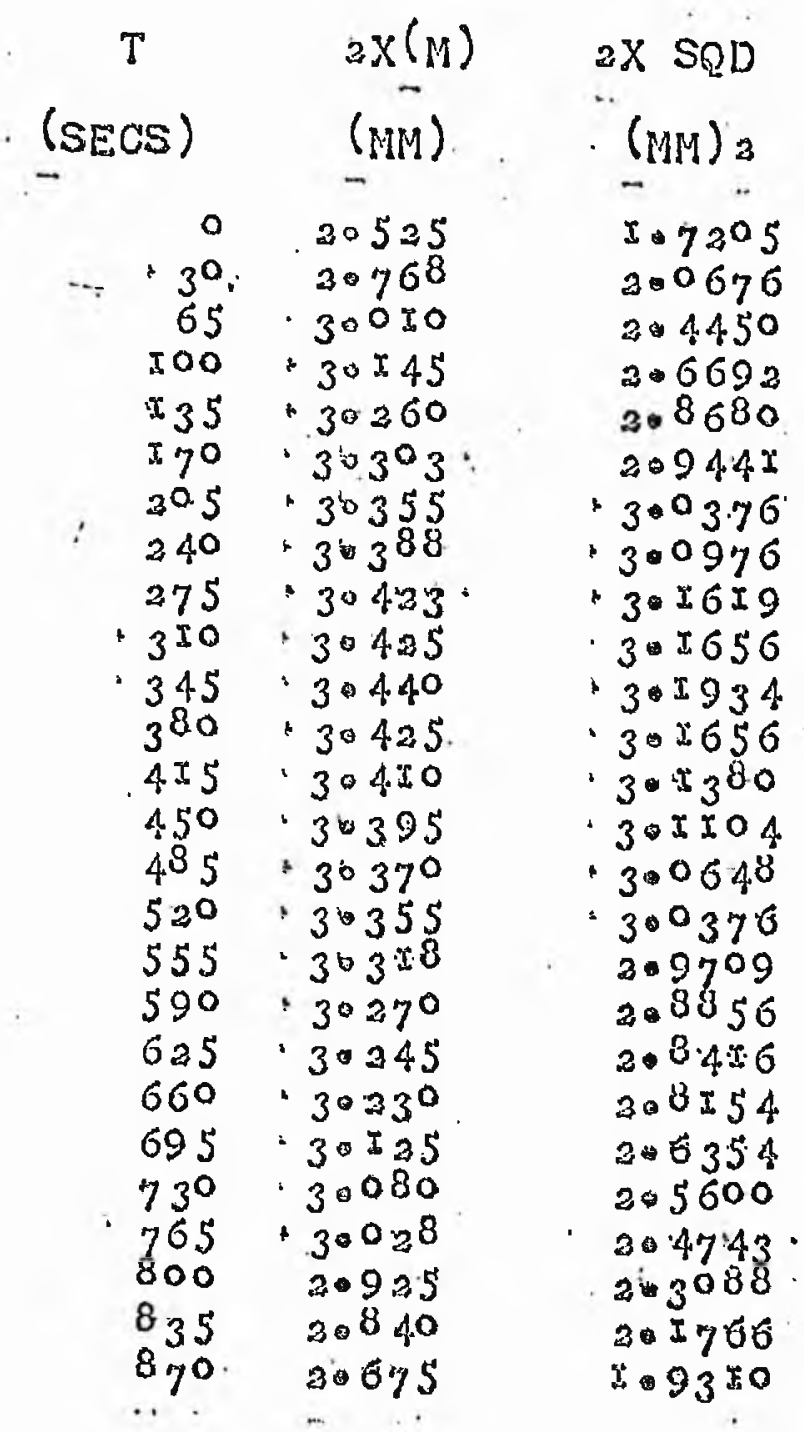




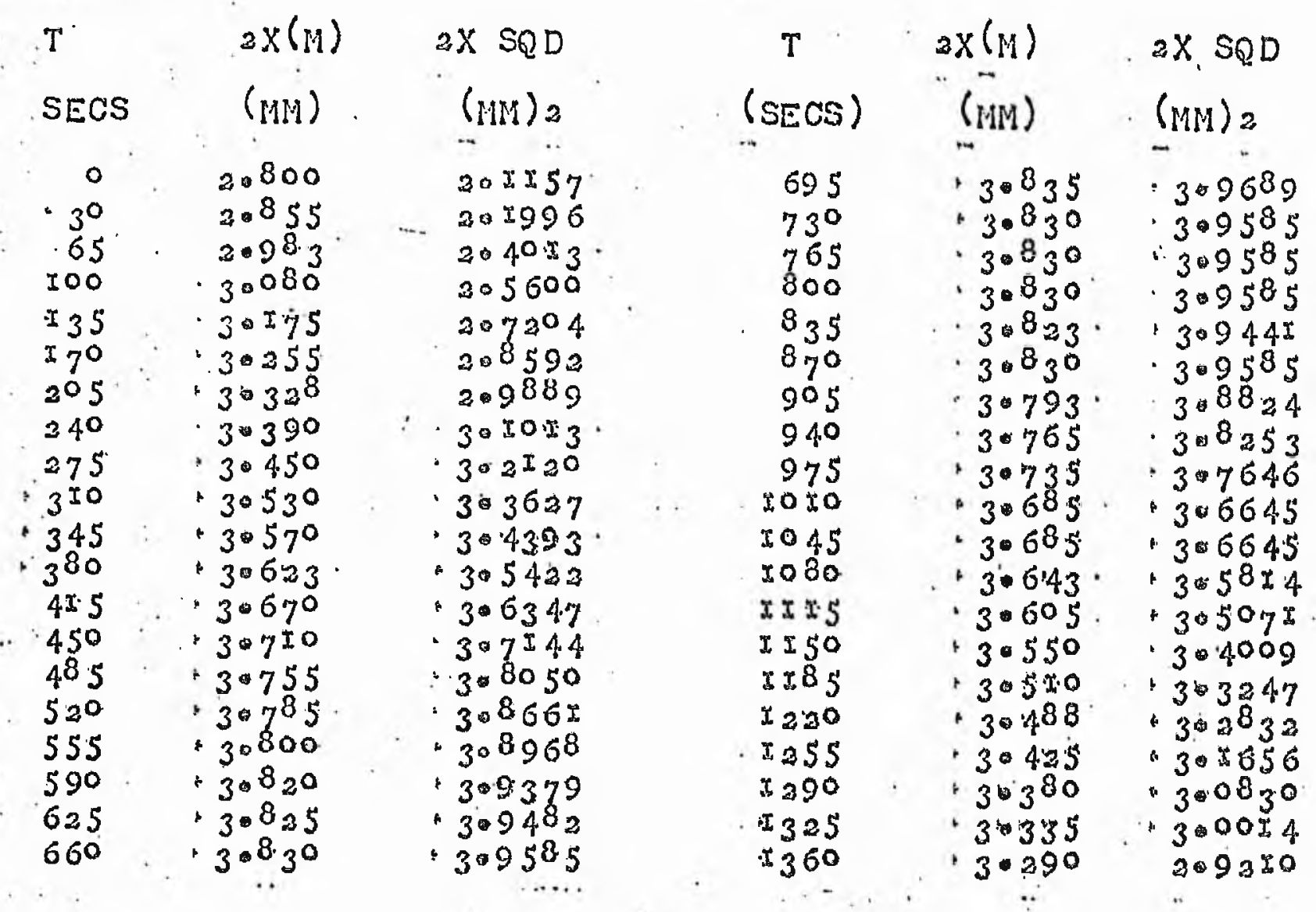


RUN NO. DEA $/ L$

0.060 WTOPCT。 DEA WATER

$$
\text { ข } 435 \uplus 3 \mathrm{DEG} \mathrm{G}_{\oplus} \mathrm{O}
$$

F.P:

\begin{tabular}{|c|c|c|}
\hline $\mathrm{T}$ & $2 \times(\mathrm{MI})$ & $\triangle X S Q D$ \\
\hline SECS? & (MM) & $(M M)_{2}$ \\
\hline$I_{70}$ & 0.833 & 302839 \\
\hline $\begin{array}{r}205 \\
-240\end{array}$ & $\begin{array}{l}0.855 \\
0.894\end{array}$ & $\begin{array}{r}303063 \\
252 x 5\end{array}$ \\
\hline 275 & 0.959 & $3 * 6645$ \\
\hline+320 & 0.936 & 207640 \\
\hline 345 & 0.9 & 209075 \\
\hline $33^{80}$ & 0.963 & $3=9357$ \\
\hline .425 & 0.9 & 300672 \\
\hline $\begin{array}{l}450 \\
485\end{array}$ & 0.9 & $\begin{array}{l}302397 \\
305486\end{array}$ \\
\hline 520 & \pm 0 & +30.47 \\
\hline 555 & \pm 0 & +300056 \\
\hline 590 & $x_{0} 0 x^{2}$ & $3 \cdot 23$ \\
\hline $66^{\circ}$ & $x * 0$ & $3028_{3}$ \\
\hline 695 & 20008 & 302024 \\
\hline $73^{\circ}$ & 20000 & $\cdot 3+549$ \\
\hline $\begin{array}{l}765 \\
800\end{array}$ & 0.998 & 30543 \\
\hline${ }^{8} 35$ & 0.938 & $\begin{array}{r}300796 \\
.30096\end{array}$ \\
\hline 870 & 0.9 & 209562 \\
\hline 905 & $0.95^{3}$ & 208954 \\
\hline 940 & 0.945 & . $\quad 308 \pm 74$ \\
\hline $\begin{array}{r}975 \\
1080\end{array}$ & $\begin{array}{l}0.935 \\
0.88 .5\end{array}$ & $\begin{array}{l}3094 \\
3049100\end{array}$ \\
\hline 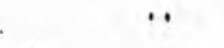 & $\ldots$ & \\
\hline
\end{tabular}

G $(C M a / \operatorname{SEC} \times 20-6)=$ ACCURACY: $:+1, \mathrm{~m}$ DELTA T (SECS) $0.3^{\circ} 63^{\circ 0}$

$6+644^{2} \cdots$

0.009 


\section{RUN NO DEA/3}

54.5 GO/LITRE DEA I WATER

$$
T=25 \cdot 3 \cdot D E G_{0} C
$$

F.P.2

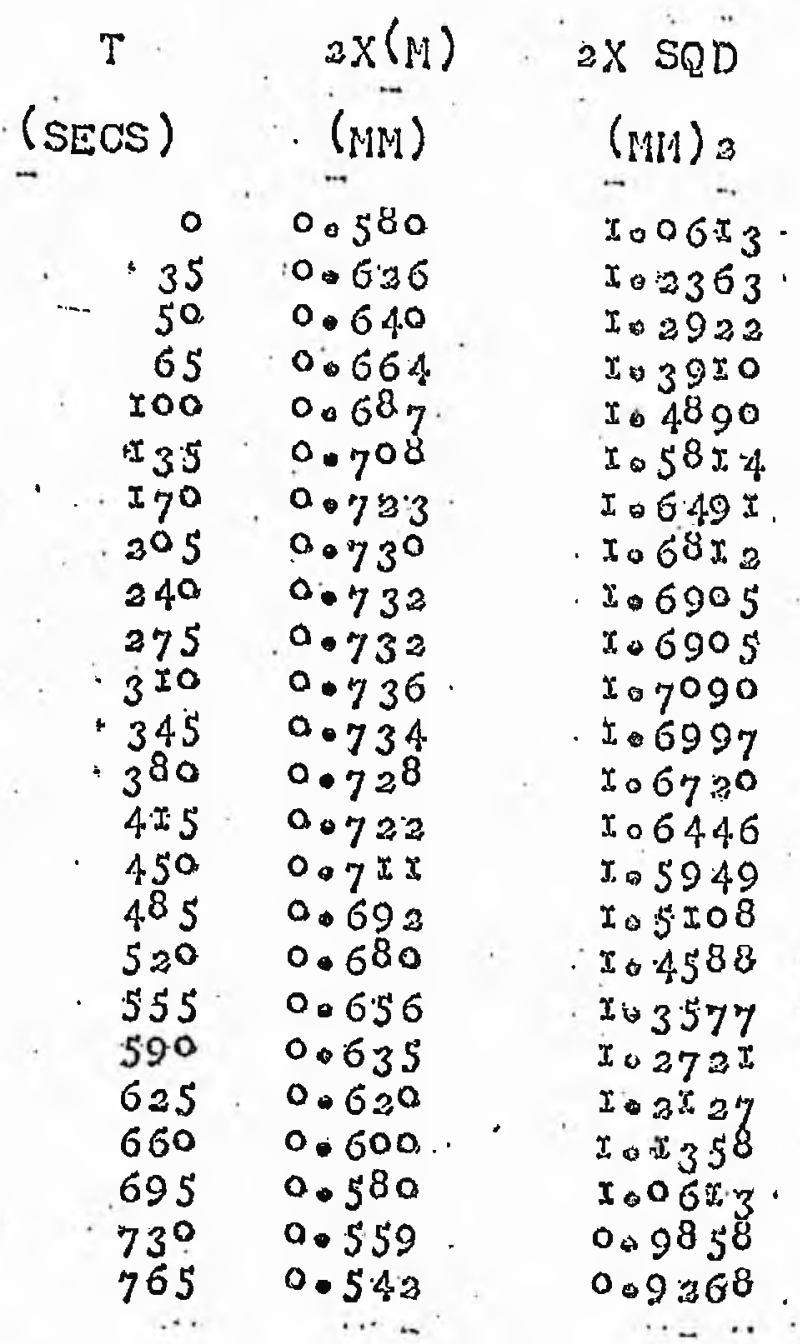


509 GO/LITRE DEA WATER

$$
\text { Fopar }
$$

\begin{tabular}{|c|c|c|}
\hline $\mathrm{T}$ & $2 x(M)$ & $2 X S Q D$ \\
\hline (SECS) & (IM) & $(\text { III })_{2}$ \\
\hline I $7^{\circ}$ & .0 .689 & so 4977 \\
\hline 205 & $0 \circ 7_{3}$ & $I_{0} 60_{3}$ \\
\hline 240 & 0.734 & x०6997 \\
\hline 275 & 0.736 & Iot 090 \\
\hline $3 \pm 0$ & 0.741 & $\operatorname{Iog} 323$ \\
\hline 345 & 00747 & 507605 \\
\hline & 0.74 .4 & 507463 \\
\hline $42-5$ & 0.743 & 207456 \\
\hline $45^{\circ}$ & 0.743 & $x \circ 737^{\circ}$ \\
\hline $4^{0} 5$ & 0.744 & $x_{07} 463$ \\
\hline $5 z^{\circ}$ & $0.73^{\circ}$ & $206^{8} x_{2}$ \\
\hline 555 & $0.7^{2} 2$ & 205993 \\
\hline 590 & $0.7^{0} 4$ & $\pi 05636$ \\
\hline $\begin{array}{l}625 \\
660\end{array}$ & $\begin{array}{l}0.702 \\
0.630\end{array}$ & $\begin{array}{l}\text { I. } 5547 \\
\text { Io } 4588\end{array}$ \\
\hline 695 & 0.674 & 204332 \\
\hline $73^{\circ}$ & 0.643 & I0 $3^{\circ} 44$ \\
\hline $\begin{array}{l}765 \\
800\end{array}$ & 00625 & 502324 \\
\hline 835 & $a_{059}$ & $\begin{array}{l}101607 \\
\text { xoग020 }\end{array}$ \\
\hline $87^{\circ}$ & 0.574 & I.0.395 \\
\hline 905 & 0.5 & 009064 \\
\hline 940 & 0 & $0 . \eta^{88} 7$ \\
\hline 975 & 0.473 & $007^{0} 39$ \\
\hline
\end{tabular}


I54.5 G./LITRE DEA WATER

$T=25 \cdot 3 \quad D E G \cdot C$

$F \bullet P \bullet 2$

\begin{tabular}{|c|c|c|c|c|c|}
\hline$T$ & $3 \times(11)$ & $2 X$ SQD & $T$ & $2 \times(M)$ & $2 X$ SQD \\
\hline SECS & (min) & $(\mathrm{riM})_{2}$ & (SECS) & (MH) & $(M M)_{2}$ \\
\hline 100 & $I .980$ & $3 \cdot 3$ III & $122^{\circ}$ & $2 \cdot 92^{8}$ & 5.0539 \\
\hline I35 & $\begin{array}{l}2.135 \\
2.260\end{array}$ & $\begin{array}{r}20687 I \\
.30100\end{array}$ & $I 255$ & 2.945 & 5 - II 28 \\
\hline 205 & $2 \cdot 335$ & $\begin{array}{l}30109 \\
3 \cdot 2 I 4 I\end{array}$ & $\begin{array}{l}1290 \\
-1325\end{array}$ & $\begin{array}{l}2.920 \\
2.933\end{array}$ & $\begin{array}{l}5 \cdot 0263 \\
5 \cdot 07 x_{2}\end{array}$ \\
\hline 240 & 2.440 & $3 \cdot 5097$ & \pm 360 & $\begin{array}{l}2.933 \\
2.900\end{array}$ & $\begin{array}{l}5 \cdot 0712 \\
4.9577\end{array}$ \\
\hline 275 & $2 \cdot 5 I 8$ & $\cdot 3 \cdot 737^{6}$ & 1395 & 2.893. & $4.933^{8}$ \\
\hline & $2 \cdot 555$ & $3.84^{8} 3$ & $143^{\circ}$ & 2.875 & $4 \cdot 8726$ \\
\hline $\begin{array}{l}345 \\
380\end{array}$ & $2 \cdot 6$ IS & $4 \cdot 0_{3}$ II & 1465 & $2 \cdot 83^{8}$ & $4 \cdot 7480$ \\
\hline $3^{80}$ & $2 \cdot 693$ & $4 \cdot 2752$ & 1500 & $2 \cdot 83^{8}$ & $4 \cdot 74^{8} 0$ \\
\hline $4 I 5$ & $2 \cdot 710$ & $4 \cdot 3294$ & 1535 & 2.888 & $4 \cdot 9168$ \\
\hline $45^{\circ}$ & $2 \cdot 745$ & $4 \cdot 4419$ & $157^{\circ}$ & 2.888 & $4 \cdot 9168$ \\
\hline $4^{85}$ & $2 \cdot 75^{8}$ & $4 \cdot 4^{8} 4 I$ & 1605 & $2 \cdot 833$ & $4 \cdot 73^{I} 3$ \\
\hline $\begin{array}{l}52^{\circ} \\
555\end{array}$ & $\begin{array}{l}2.803 \\
2.808\end{array}$ & $4 \cdot 63=6$ & $164^{\circ}$ & 2.840 & $4 \cdot 7547$ \\
\hline $\begin{array}{l}555 \\
590\end{array}$ & $\begin{array}{l}2.808 \\
2.835\end{array}$ & $4 \cdot 64^{8} I$ & 1675 & $2 \cdot 7^{80}$ & $4 \cdot 5559$ \\
\hline 625 & 2.840 & $\begin{array}{l}4 \cdot 7046 \\
4 \cdot 7547\end{array}$ & $\begin{array}{l}\text { I7 I0 } \\
\text { I } 455\end{array}$ & 2.753 & $4 \cdot 467^{8}$ \\
\hline $66^{\circ}$ & $2 \cdot 867$ & $4 \cdot 8455$ & $\begin{array}{l}1745 \\
1780\end{array}$ & $\begin{array}{l}2 \cdot 723 \\
2.728\end{array}$ & $\begin{array}{l}4 \cdot 3710 \\
4 \cdot 387\end{array}$ \\
\hline 695 & $2 \cdot 883$ & 4.8997 & I8 55 & 2.730 & $4 \cdot 36 I 4$ \\
\hline $73^{\circ}$ & $3 \cdot 92^{\circ}$ & $5 \cdot 0_{2} 6_{3}$ & 1850 & $2 \cdot 67^{8}$ & $4 \cdot 2277$ \\
\hline $\begin{array}{l}765 \\
800\end{array}$ & 2.915 & 5.0091 & 1885 & $2 \cdot 620$ & 4.0466 \\
\hline $\begin{array}{l}800 \\
835\end{array}$ & $2 \cdot 92$ & $5 \cdot 0_{2} \sigma_{3}$ & $192^{\circ}$ & $2 \cdot 600$ & $3 \cdot 9^{8} 50$ \\
\hline $\begin{array}{l}35 \\
870\end{array}$ & 2.920 & $5 \cdot 0_{2} \sigma_{3}$ & 1955 & 2.573 & $3 \cdot 9027$ \\
\hline 905 & $\begin{array}{l}2.940 \\
2.948\end{array}$ & 5.0954 & 1990 & $\begin{array}{l}2.535 \\
2.500\end{array}$ & $3 \cdot 7883$ \\
\hline 1045 & $2.92^{8}$ & $\begin{array}{l}5.1232 \\
5.0539\end{array}$ & $\begin{array}{l}2025 \\
2060\end{array}$ & $\begin{array}{l}2.500 \\
2.50_{3} .\end{array}$ & $\begin{array}{l}306044 \\
30.69322\end{array}$ \\
\hline & $2 \cdot 93^{8}$ & 5.0885 & 2095 & 2.465 & +3.5819 \\
\hline IIIS & $2 \cdot 943$ & $5 \cdot$ & $2^{ \pm} 3^{\circ}$ & $2 \cdot 323$ & $3 \cdot 18 I I$ \\
\hline $5^{\circ}$ & 2.918 & $5 \cdot 0194$ & $227^{\circ}$ & 2.235 & 2.9447 \\
\hline & 2.943 & $5 \cdot 1058$ & $23^{\circ} 5$ & $2 \cdot I 43$ & $2 \cdot 7^{\circ} 73$ \\
\hline
\end{tabular}

CALCULATED....

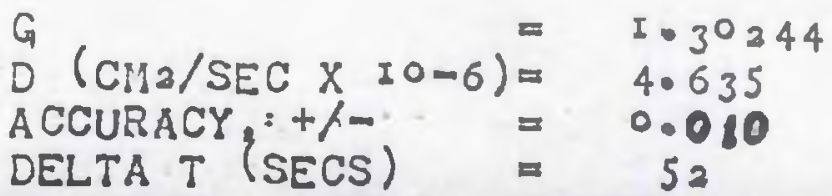




\section{Fop:3}

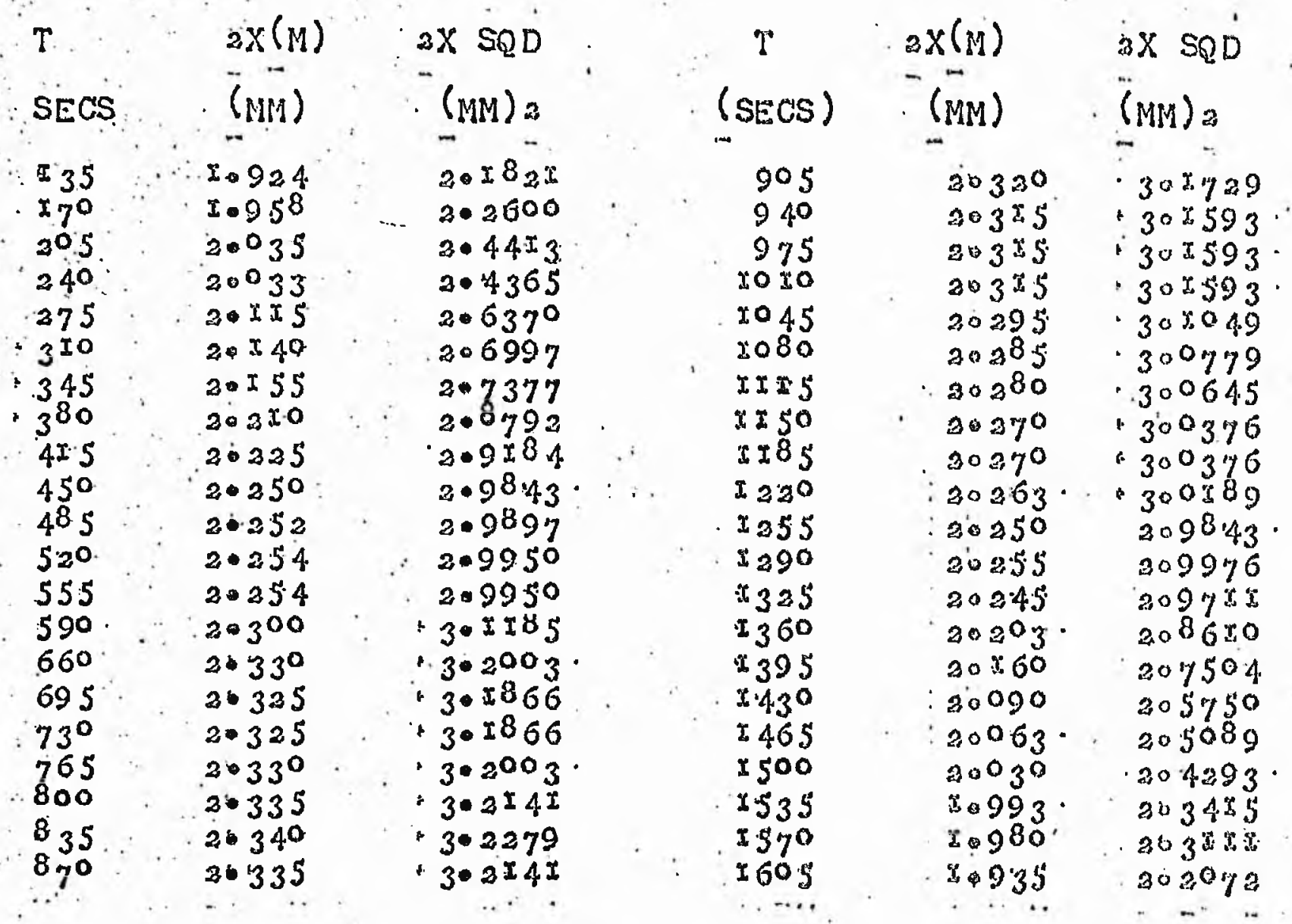

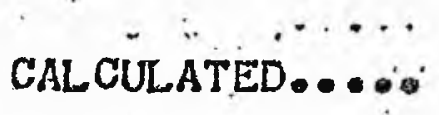


347. IS G./LITRE DEA UATER

$$
T=25 \cdot 3 \cdot D E G \cdot C
$$

\begin{tabular}{|c|c|c|}
\hline$T$ & $2 \times(M)^{6}$ & $2 \times S \Omega D$ \\
\hline SECS & $(M M)$ & $($ (m!) $)$ \\
\hline 170 & 1.765 & \\
\hline 205 & 1.815 & \\
\hline 210 & 1.905 & $2 \cdot 3$ \\
\hline$=75$ & $I .3^{80}$ & \\
\hline $\begin{array}{l}310 \\
345\end{array}$ & $\begin{array}{l}2.033 \\
2.088\end{array}$ & 2. \\
\hline $\begin{array}{l}345 \\
380\end{array}$ & $\begin{array}{l}2.088 \\
2.115\end{array}$ & $\begin{array}{l}2 \cdot 7 \\
2.8\end{array}$ \\
\hline $4 I 5$ & 2.150 & 2.360 \\
\hline 450 & 2.193 & 3.086 \\
\hline 485 & 2.218 & $3 \cdot I$ \\
\hline 520 & 2.255 & $3 \cdot 2$ \\
\hline 555 & 2.275 & $3 \cdot 3$ \\
\hline 590 & $2 \cdot 3$ I & $3 \cdot$ \\
\hline $\begin{array}{l}625 \\
660\end{array}$ & $2 \cdot 343$ & ? \\
\hline $\begin{array}{l}660 \\
695\end{array}$ & 2.355 & $3 \cdot$ \\
\hline 730 & $\begin{array}{l}2.383 \\
2.395\end{array}$ & $\begin{array}{l}30.94 \\
3.58\end{array}$ \\
\hline 765 & $2 . \div 25$ & $2 \cdot 7$ \\
\hline 800 & 2.435 & \\
\hline 835 & 2.465 & $3 \cdot 2^{\circ}$ \\
\hline 370 & 2.475 & ¿ \\
\hline 905 & 2.505 & $\because \gamma$ \\
\hline 940 & $2.5=0$ & $40: 4$ \\
\hline 975 & $2 \cdot 550$ & $\begin{array}{l}4=108 \\
\therefore: 103\end{array}$ \\
\hline $\begin{array}{l}1010 \\
1045\end{array}$ & $\begin{array}{l}2 \cdot 5 j 0 \\
2 \cdot 543\end{array}$ & 4.13 \\
\hline 1080 & $\begin{array}{l}2.543 \\
2.545\end{array}$ & $4: I ?$ \\
\hline IIs & $2 \cdot 548$ & $\therefore 15$ \\
\hline I I 50 & 2.555 & U. I \\
\hline & 2.565 & $\hat{0} \cdot 2=$ \\
\hline
\end{tabular}

$\begin{array}{ccc}T & 2 \times(M) & 2 \times \text { SQD } \\ \text { (SECS) } & \text { (Mi) } & (\text { (Min) } 2\end{array}$

I 220

$x=55$

I 290

1325

1360

1395

I 430

1465

1500

I 535

I 570

I 605

1640

1675

I 7 IO

I 8 I

I 850

I 950

$20=5$

$\therefore i \frac{3}{2.8}$

2 I

2165

2200

2235

2270

2305

2340

2375

3445
2.575

2.588

$2.5^{8} 5$

2.600

2.590

$2 \cdot 570$

$2 \cdot 598$

3.585

3.585

$2 \cdot 570$

$2 \cdot 548$

2.550

2.540

2.540

2.530

$2.5 I 5$

2.510

2.487

2.425

2.425

2.440

2.423

2.410

2.410

2.400

2.390

$-380$

2.338

2.328
$4.255^{8}$

4.2558

4.2989

4.2890

4.3389

$4 \cdot 3056$

4.2393

$4 \cdot 3322$

4.2830

4.3890

4.2393

4. $167 I$

4. 1736

4.1409

4. 1409

4. 1084

4.0598

4.0437

3.9715

3. 7745

3.7745

$3 \cdot 8213$

$3 \cdot 7682$

3.7279

3.7279

3.6970

3.6663

3.6357

3.5085

$3 \cdot 4785$

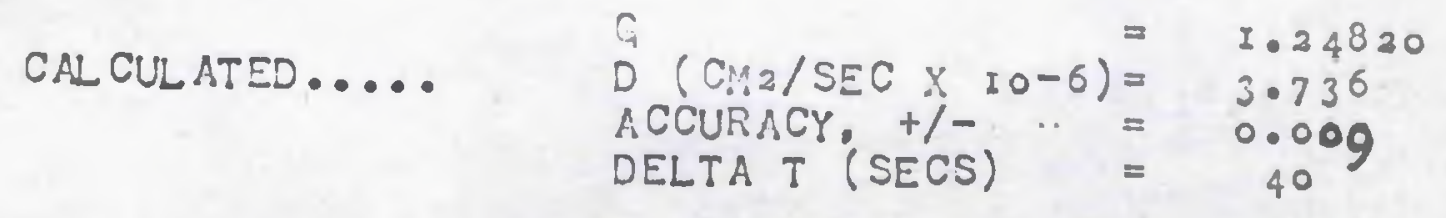


347.IS G./LITRE DEA WATER

$$
T=25.3 D E G_{0} C
$$

F.P. 4

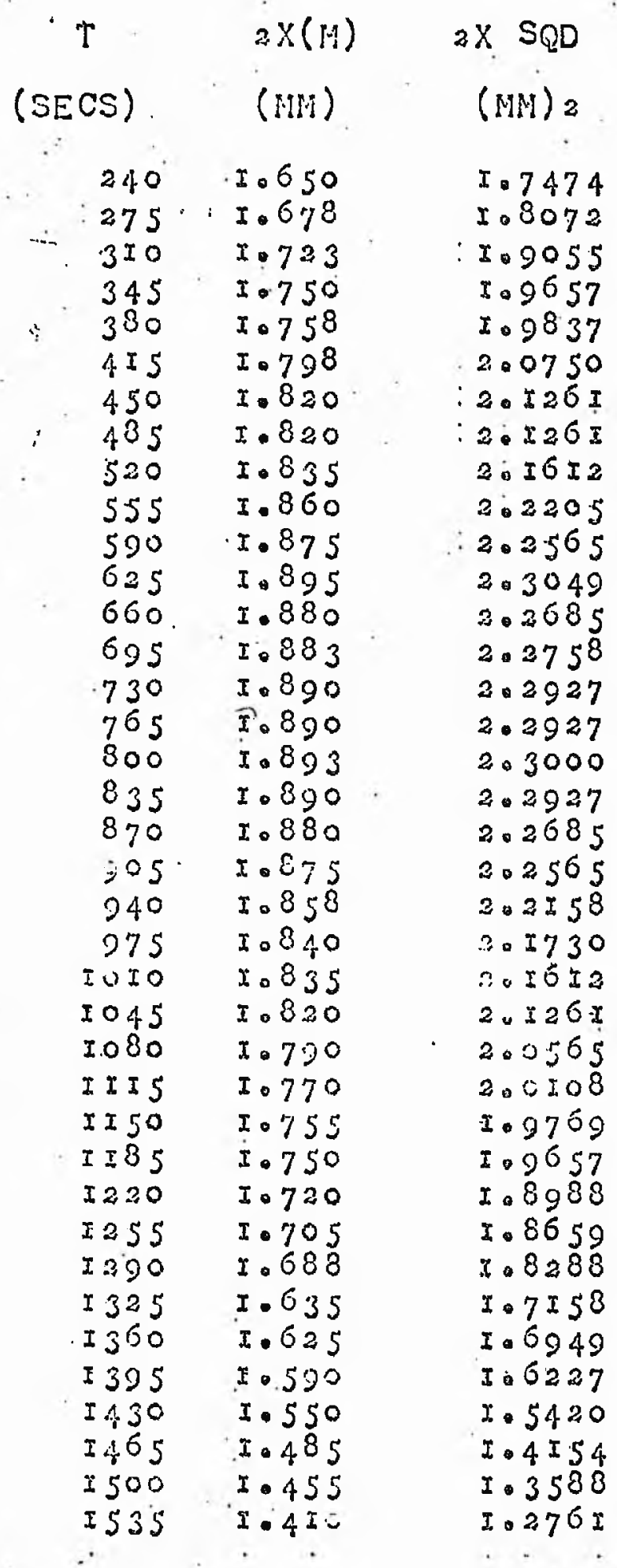

CAL CULATED

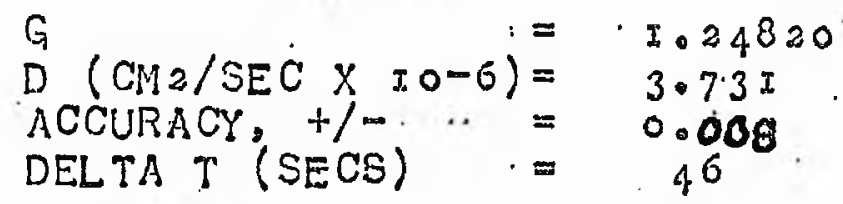


46205 Go/LITRE DEA VATER

$$
\text { FoP. } 4
$$

\begin{tabular}{|c|c|c|c|c|c|}
\hline$T$ & $2 \times(11)$ & $2 X S Q D$ & $T$ & $2 X(M)$ & $2 X \quad S Q D$ \\
\hline SECS & (HM) & $(M M)_{2}$ & (SECS) & (MM) & $(M M)_{2}$ \\
\hline $\begin{array}{l}x 70 \\
240\end{array}$ & $\begin{array}{l}x \cdot 398 \\
I .580\end{array}$ & $\begin{array}{l}I 02544 \\
I_{0} 6023\end{array}$ & $\begin{array}{l}905 \\
940\end{array}$ & $\begin{array}{l}10773 \\
10758\end{array}$ & $\begin{array}{l}200 I 77 \\
x \circ 9837\end{array}$ \\
\hline $\begin{array}{l}275 \\
310\end{array}$ & $\begin{array}{l}10633 \\
50665\end{array}$ & $\begin{array}{l}\text { I. } 7216 \\
\text { I.7793 }\end{array}$ & $\begin{array}{r}975 \\
10 \pm 0\end{array}$ & $\begin{array}{l}x \circ 740 \\
x 0740\end{array}$ & $\begin{array}{l}x \circ 9.433 \\
x \circ 9433\end{array}$ \\
\hline $\begin{array}{l}345 \\
380\end{array}$ & $\begin{array}{l}I 069^{\circ} \\
I 072^{\circ}\end{array}$ & $\begin{array}{l}I_{0} 8332 \\
I_{0} 8988\end{array}$ & $\begin{array}{l}1045 \\
1080\end{array}$ & $\begin{array}{l}\text { I0720 } \\
\text { I0755 }\end{array}$ & $\begin{array}{l}\text { I. } 8988 \\
\text { I.8878 }\end{array}$ \\
\hline $4 \times 5$ & $\operatorname{I0733}$ & $I \circ 9277$ & I. 25 & I0 690 & I. 8332 \\
\hline $45^{\circ}$ & 50765 & $I .9995$ & I I 50 & Io $67^{\circ}$ & $x 07900$ \\
\hline $\begin{array}{l}4^{8} 5 \\
500\end{array}$ & 50755 & $I \circ 9769$ & $1 \pm 85$ & 20650 & 3.7474 \\
\hline $\begin{array}{l}520 \\
555\end{array}$ & $\begin{array}{l}\text { I. } 780 \\
\text { I0 } 790\end{array}$ & 2.0336 & $122^{\circ}$ & $x \circ 645$ & 107369 \\
\hline $\begin{array}{l}555 \\
590\end{array}$ & $\begin{array}{l}\text { I0 } 790 \\
\text { I. } 795\end{array}$ & 2.0565 & \pm 255 & $x .62^{\circ}$ & $x \circ 6^{8} 45$ \\
\hline 625. & $\begin{array}{l}10795 \\
\text { I0795 }\end{array}$ & 200680 & $x_{290}$ & I. 605 & I. 6534 \\
\hline 660 & 10793 & 2006004 & $\begin{array}{l}325 \\
8360\end{array}$ & $\begin{array}{l}\text { I. } 585 \\
x=560\end{array}$ & $\begin{array}{l}506325 \\
x 05620\end{array}$ \\
\hline $69 \mathrm{~S}$ & $I 0795$ & 2.0680 & 39.5 & $x \circ 545$ & $50532 \mathrm{I}$ \\
\hline $73^{\circ}$ & $I_{0798}$ & $20075^{\circ}$ & $143^{\circ}$ & $x \circ 52^{\circ}$ & $x \circ 4^{6}=9$ \\
\hline 800 & $x .790$ & 200565 & 1465 & $I_{0} 4_{3}^{8}$. & Io $45 \mathrm{I} 6$ \\
\hline 835 & $2 \circ 788$ & 200519 & 500 & 20455 & Io 3588 \\
\hline & $x \circ 780$ & 200536 & 5535 & $50.43^{\circ}$ & $5 \circ 3^{48.25}$ \\
\hline
\end{tabular}

CALCULATED.

$\mathrm{G}$

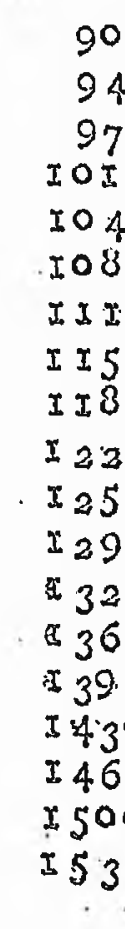

$$
T=2503 \cdot D E G_{0} C
$$




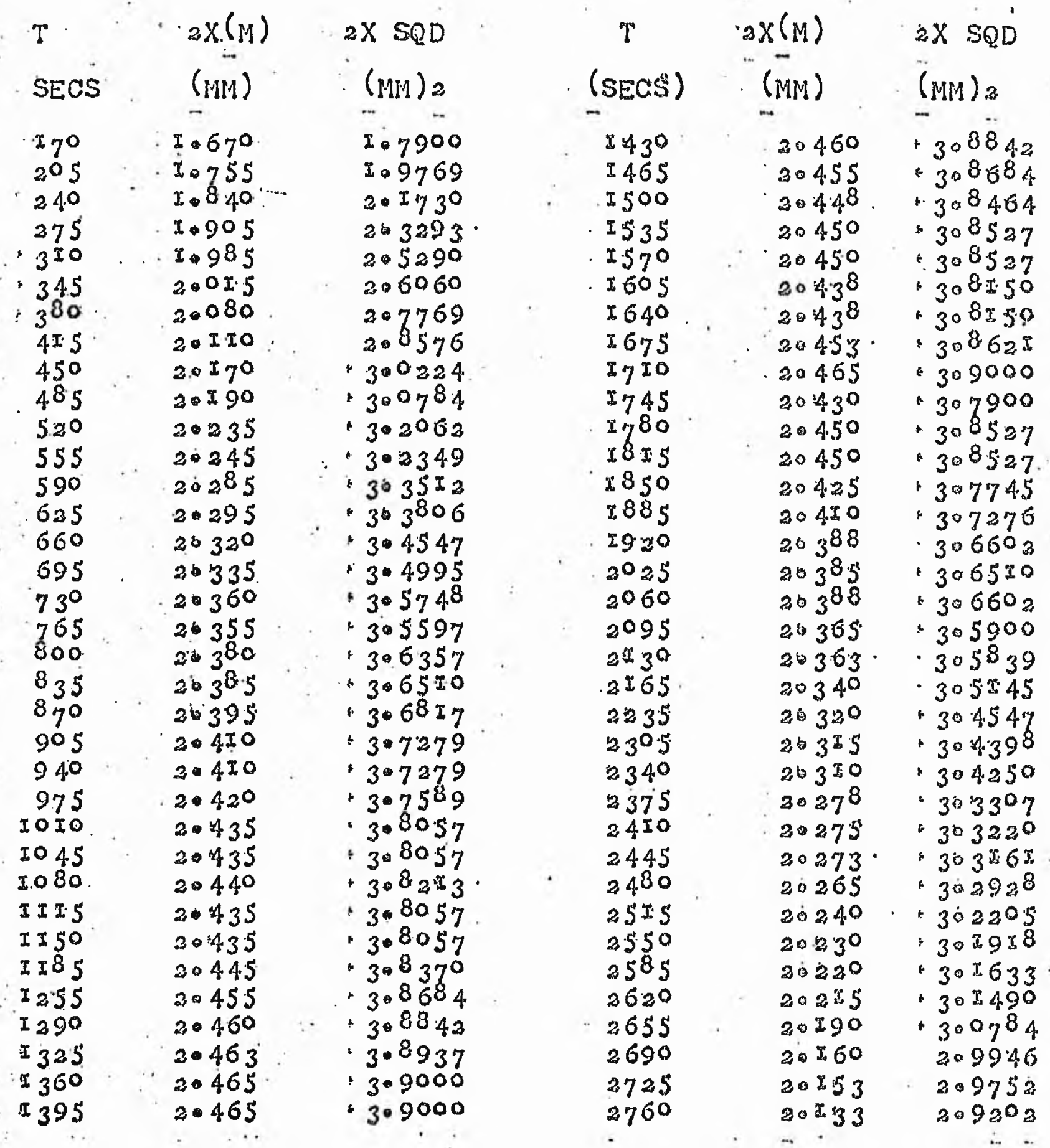




$$
T=35 \cdot 3 D E G \cdot C
$$

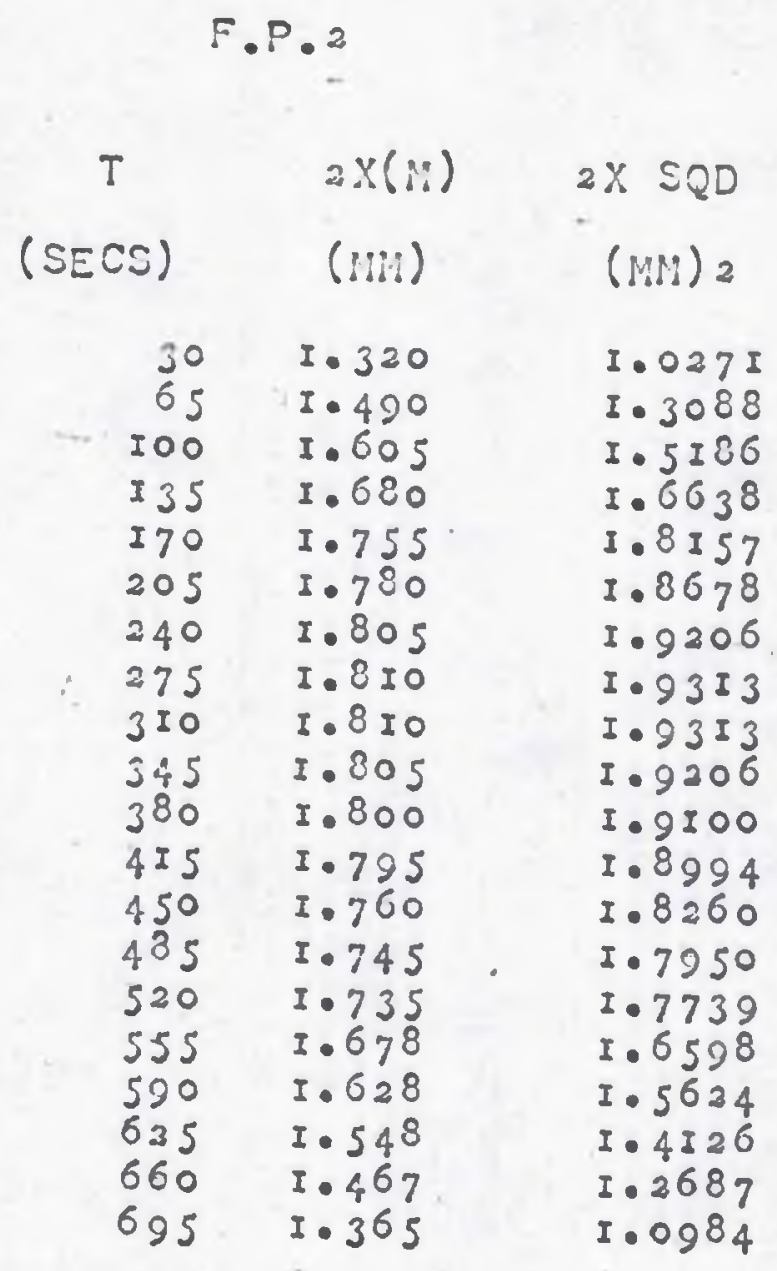




$$
T=35 \cdot 3 D E G \cdot C
$$

$$
\text { F.P:2. }
$$

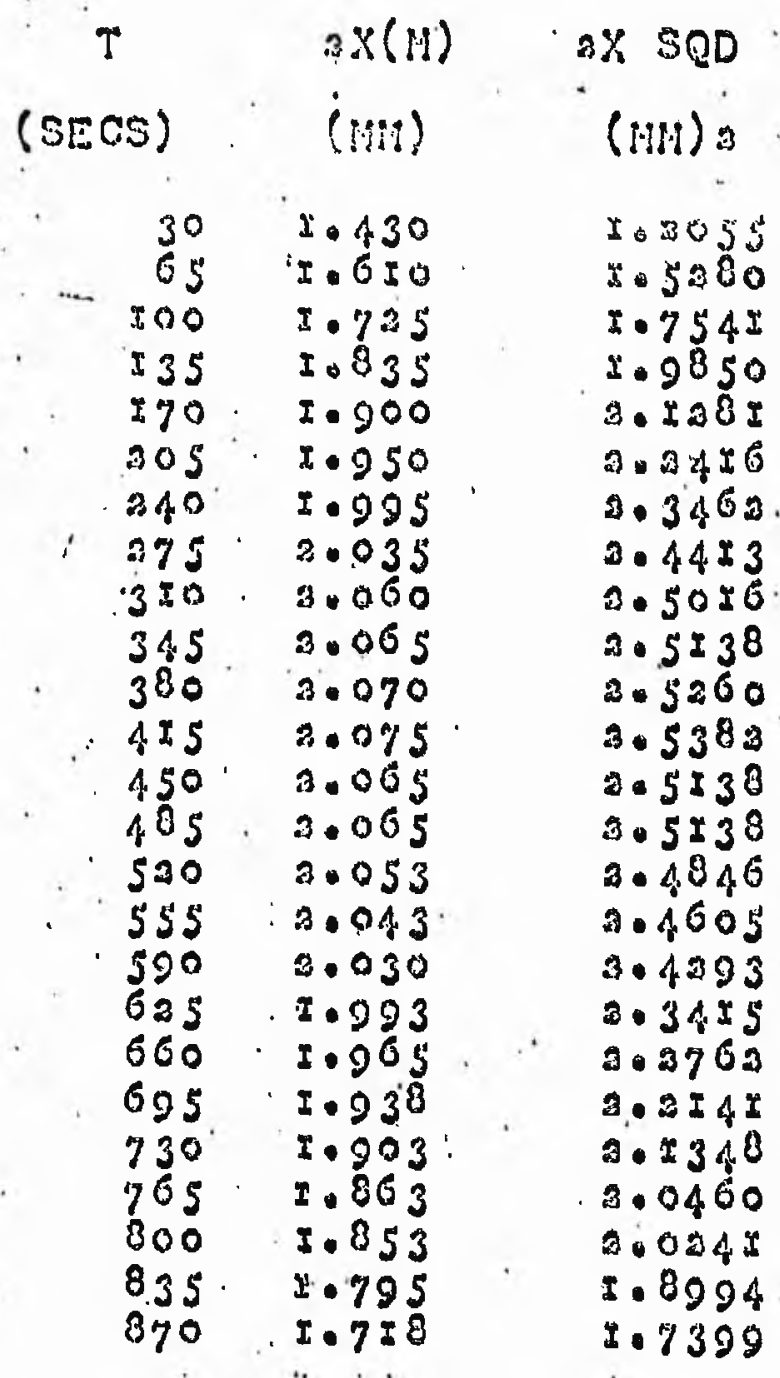


$\because$ RUN NO. TEA/3

200.56 G./LITPE TEA WATER

$T=25 \cdot 3$ DEG.C

F.P. 3

T

SECS

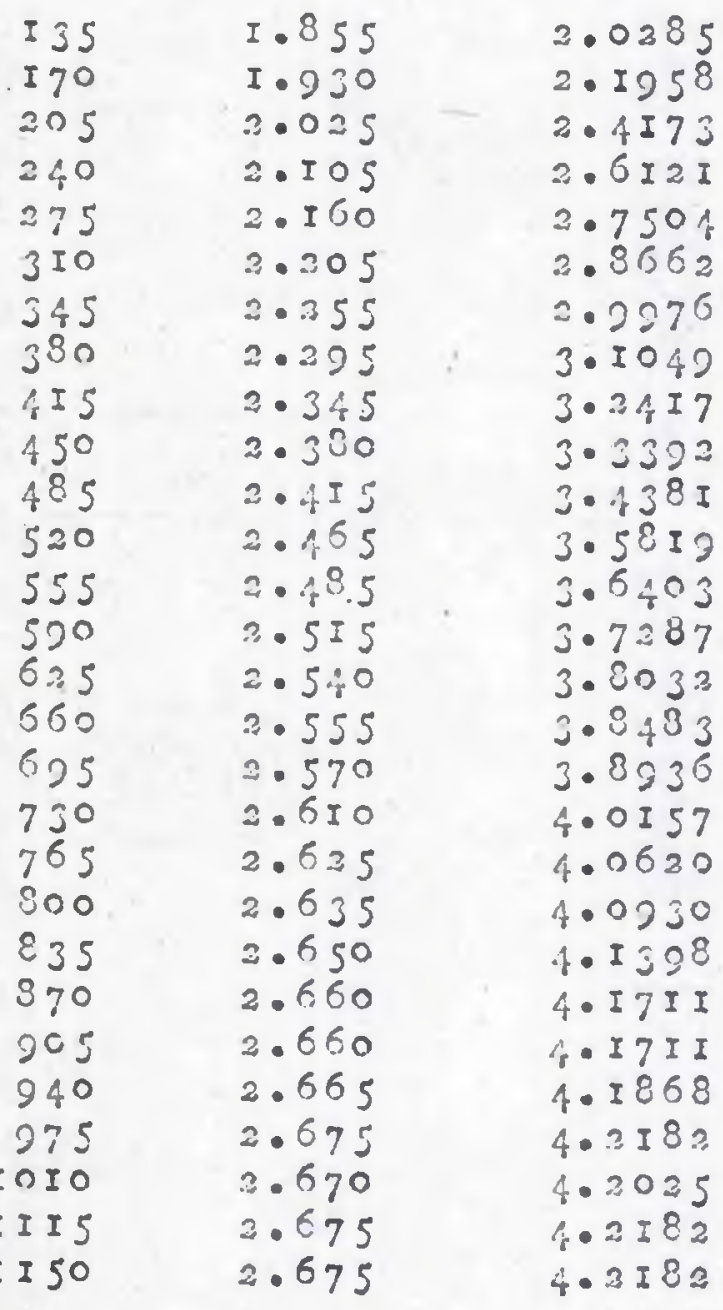

$2 \times$ SQD

(III) 2

$$
(m)
$$

1.855

I.930

.025

2.105

.355

.295

. 345

30

. 4 I I

$2 \cdot 4^{6} 5$

- 8.5

.555

.570

.610

2.635

.665

.675

2.675

.675
T

(SECS)

I I 5

I 220

1255

1290

I 325

1360

1430

1455

I 500

1535

I 570

$+605$

1640

I 675

I 7 IO

I 745

I 780

I 8 I 5

I 850

I 835

I 920

I 955

I 930

2025

2000

2095

. I 30

2. 65
$2 \times(!)$

(MM)

2.670

2.660

2.650

2.655

2.635

2.635

2.655

2.655

2.625

2.625

$2.6=0$

3.585

2.585

3.585

2.575

2. 530

2. 508

2. 470

2.430

2.405

2.385

2. 370

2.335

2.330

2. 3 I 5

2.295

2.255

$2.20 \mathrm{~S}$
$3 \times S Q D$

(Min) 2

4.2025

4. I 7 II

$4 \cdot 1398$

4.5554

4.0930

4.0930

4. 1554

$4 \cdot I 554$

4.0620

4.0620

4.0157

$3.932=$

3. 9392

3.2392

3.0088

3.7733

3.7080

$3 \cdot 5265$

3.1809

3.4097

$3 \cdot 3532$

3. 3 II 2

3. 2 I 4 I

3.2003

3.1593

3. 1049

2.9976

2. 8662

CAL CULATED.....

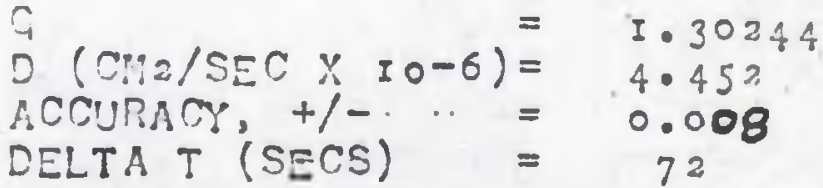


nUn : $:$. TEA/4

200.55 G./LITRE TEA MATER

$$
\text { F.P. \& }
$$

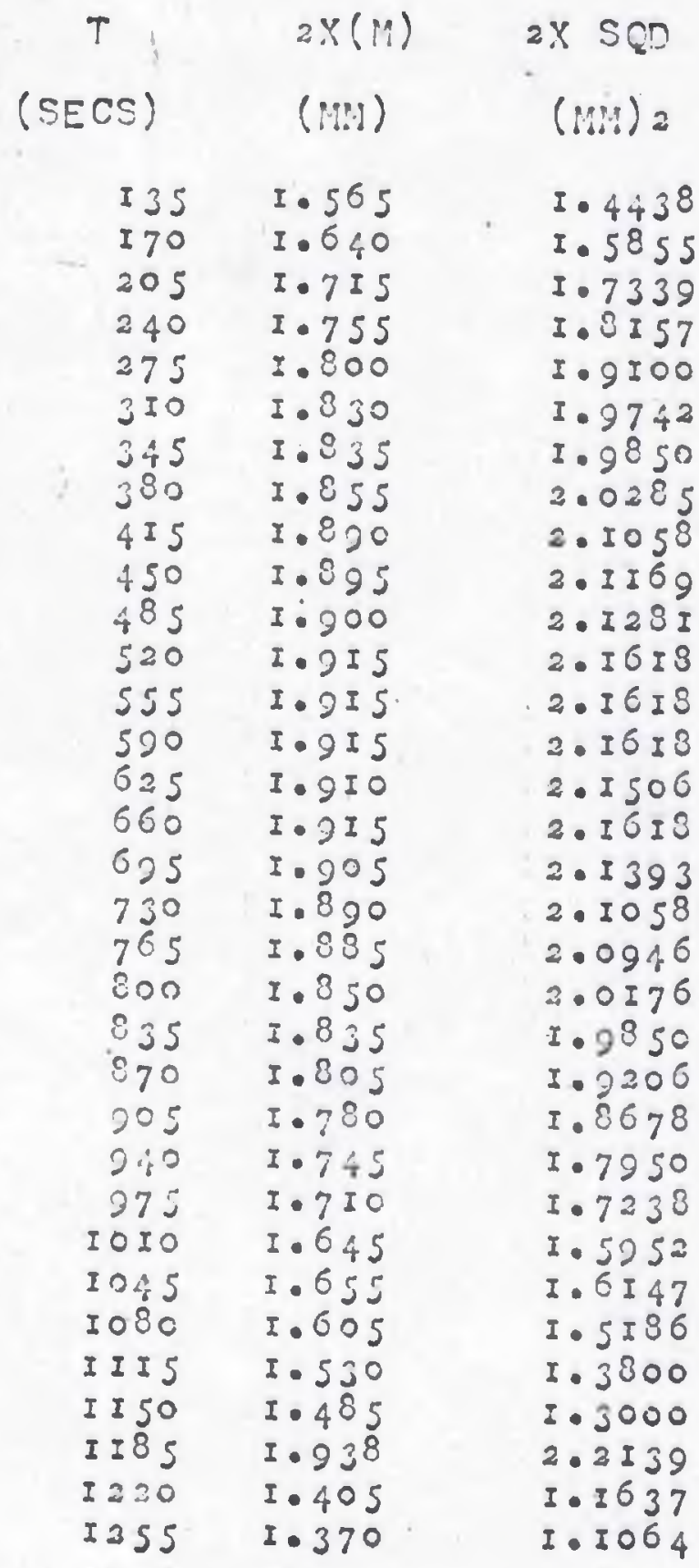

$$
T=25.3 D E G . C
$$




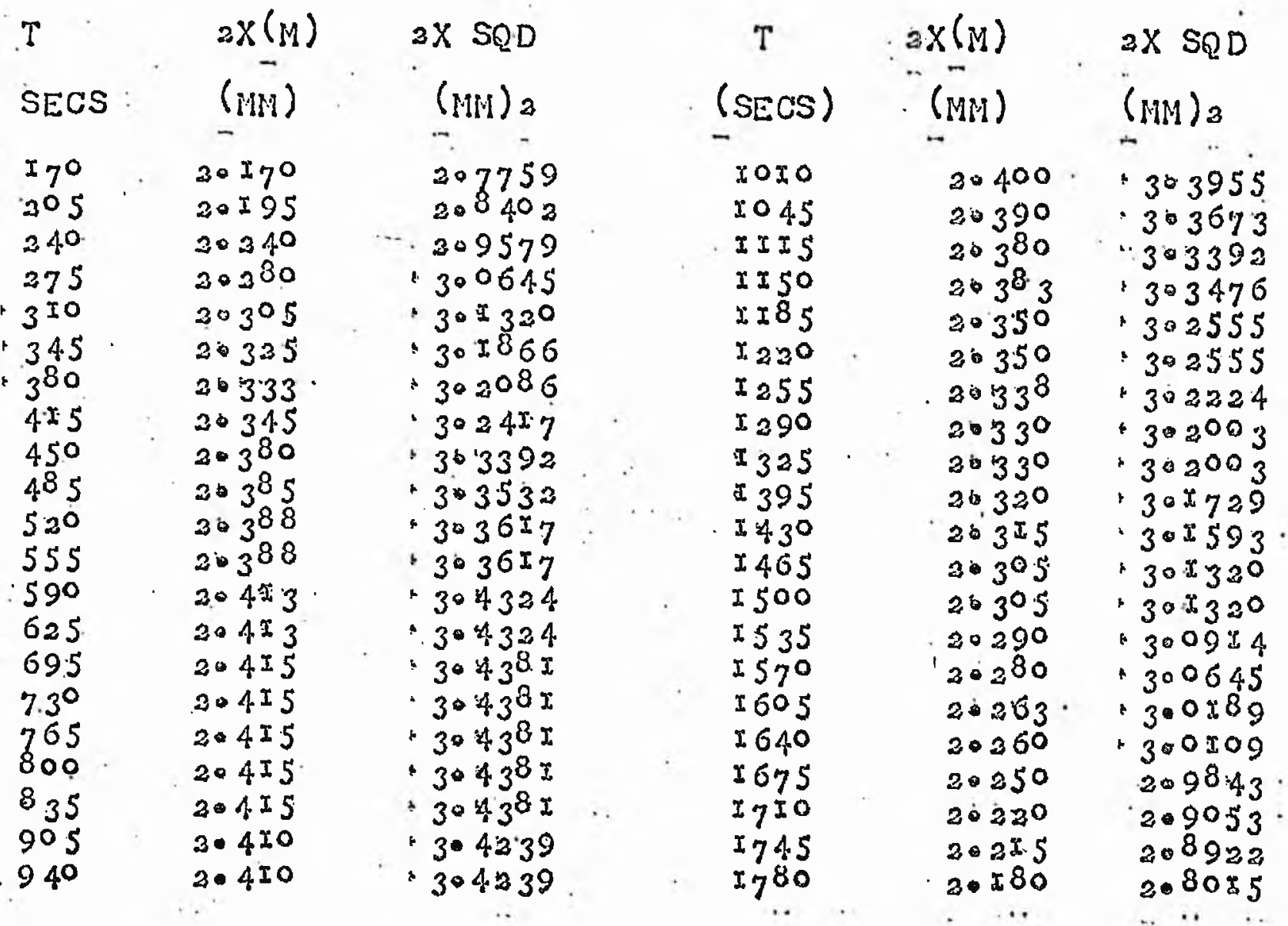

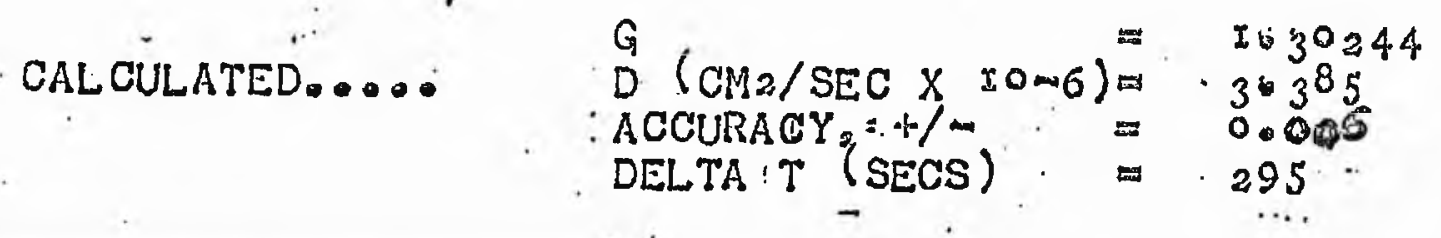


IV(d). Comparison of Results Obtained Using the Wavefront

Shearing Intenferometer, and those obtained by the Gouy Method.

Thomas and Furzer ( 1 ) ( 2 ) investigated the diffusion coefficients for the same systems as in this study. Howeveng theix results provoked critical interest from Ibrahim and Kuloor ( 3 ), who doubted the discrepancy between the experimental. Duvalues and theoretically predicted values as being due to physicomchemical effects in the ethanolaninewwater systems. There axe two Implications in Ibxahim and Truloon's criticismsg -

a) that Thomas and Furzer's D-values are suspect, and $\left.b_{i}\right)$ that the semi-empirical equations applied do not adequately describe the ethanoleminemwater diffusion process.

The latter topic will be discussed in some detail further on.

A comparison between Thomas and Furzer's resulits and the resultis obtained in this study will serve as a check on the accuracy of the former.

Although most of the exror in the work of Thomas and Furzer would appear to be due to the inherent 


\section{$-126-$}

inaccuracies of the Gouy interferometer itself, there are severaI criticisms that can be made.

As an example, calibration runs using sucrosewwater did not show a very great accuracy ( e.g. a figure of $\mathrm{D}=5.13 \times 10^{-66} \mathrm{~cm}^{2} / \mathrm{sec}$ tor one sucrose-water run is quoted without any indication of suspected inaccuracy).

On a more detajled examination of the results, no $\triangle$ t correction has been applied in any one case. Since an estimate of the $\Delta t$, and the resulting coxrected $D$-value, is of interest, Thomas and Furzer's ethanolaminemwater resultis were recalculated to include a. $\Delta$ t. A sumnary of these calculations is given, together with graphs. comparing the corrected and uncorrected Davaliues with those obtalined in this study.

\section{Zero-Time Correction Applied to the Gouy Calculations.}

In Thomas and Furzer's calculations for the Diffuision coefficients of MEA, DEA, and IEA - water, the following approach was taken; - 
The reliationship,

$$
D=\frac{\left(A_{M^{*}} \lambda \cdot b\right)^{2}}{4 \pi c_{t}^{2} \cdot t}
$$

Is. used, whexe:

$$
\begin{aligned}
& j_{m}=\text { total number of fringes in the pattem, } \\
& \lambda=\text { wavelength of light used. } \\
& b=\text { path Iength thro' diffusion cel.J. } \\
& c_{t}=Y / e^{-z^{2}} \text {, where } X \text { is the fringe displacement. } \\
& e^{-z^{2}} \text { is obitalned from a table or } f(z)_{2} e^{-z^{2}} \text {, } \\
& \mathbb{P}(z) \text { being caliculated from } f(z)=j+\frac{1}{4} \\
& j_{\text {mil }}
\end{aligned}
$$

Displacement measurements are taken for the fringes in the fringe-pattern (with the Gouy interferometer, the fringe pattern consists of a number of horizontal fringes moving downwards.). $f(z), e^{-z^{2}}$, and $c_{t}$ are then calculiated for each of : the fringes, assuming a value for $j_{m^{\circ}}$ when the stiandard. deviation of the $C_{t}$ values is a minimum, then the corresponding value of $j_{m}$ is used.

Naturaliy, $j_{m}$ should be constant throughout any: one experiment ( fringe displacements, being, measured at regulan time intervalis)。 
$D$ is thus calculated for several values of to

However, as with the wavefront shearing interferometers, a zero-time correction, / U. no nus bo app.lied to the abserved time, io (This being due to initial mixing at: the interface)。

The relationsin $\quad D=D\left(I+\frac{A t}{t}\right)$,

where $D$ is the corrected value of the diffusion coefficient, is used by Iongsworth ( 48 ). Thus a plot of $D^{\text {g }}$ against I.ft should yleld a strigaight line of slope D. Atso

Thomas and Furzer did not employ this. nelationship, but. drew instead the best streight: Iine through plots of

$$
\begin{aligned}
& \text { I) } j_{m} \text { vso } t . \\
& \text { 2) } I_{t} / C_{t}^{2} \text { vsis } 1 / t
\end{aligned}
$$

the intercepts yielding $j_{m}$ and ${ }^{I} / C_{t}^{2}$ respectivelyg and from these values D was calculated.

For the purpose of determining the accuracy of the Gouy resultis, Iongsworthls method for calculating $D$ and A t was applied to Thomas and Furzers sesultis. These calculations are summarised in table 4.

Thomas; and Furzers's resultis, with a) no $A$ t applied, and b) Lt considered, are: compared graphically with the resultis obtained using the wavefront shearing intenferometer in Figs. 18, 1.9, and 20. 
Tablie 40

Calcuilation of $\Delta t$ for Thomas, and Furzer's Results.

\begin{tabular}{|c|c|c|c|c|c|}
\hline \multirow[t]{2}{*}{ Run. No. } & \multicolumn{2}{|c|}{ Solute Concn. } & \multirow{2}{*}{$\begin{array}{c}\mathrm{cm}^{2} / \mathrm{sec} \\
\times 10^{6}\end{array}$} & \multirow{2}{*}{$\begin{array}{c}\mathrm{D} \\
\mathrm{cm}^{2} / \mathrm{sec}^{6} \\
\times \mathrm{m}^{6}\end{array}$} & \multirow{2}{*}{$\begin{array}{r}t \\
\operatorname{secs}\end{array}$} \\
\hline & mli/litre & $\mathbb{N}$ & & & \\
\hline MEA I & 7 & 0.12 & 10.8 & 10.08 & 94 \\
\hline 2 & 40 & 0.67 & 10.2 & 10.04 & 9 \\
\hline 3 & 300 & 1.67 & 8.52 & 8.17 & 89 \\
\hline 4 & 200 & $3 \cdot 34$ & 5.11. & 4.65 & 738 \\
\hline DEA 3 & 40 & 0.42 & 5.67 & 5.54 & 24 I \\
\hline 2 & 100 & 1.05 & 4.64 & 4.74 & 170 \\
\hline 3 & 200 & 2.10 & 3.82 & 3.79 & 248 \\
\hline 4 & 400 & 4.19 & 2.81 & 2.26 & 2067 \\
\hline 5 & 500 & 5042 & 2.28 & 2.22 & 497 \\
\hline TEA 1 & 4 & 0.03 & 7.07 & 8.38 & $-4+2$ \\
\hline 2. & 20 & 0.15 & 6.57 & 6.24 & 106 \\
\hline 3 & 200 & 1.52 & 4.22 & 3.093 & 239 \\
\hline 4 & 400 & 3.01 & 1.70 & 1.42 & 2339 \\
\hline
\end{tabular}



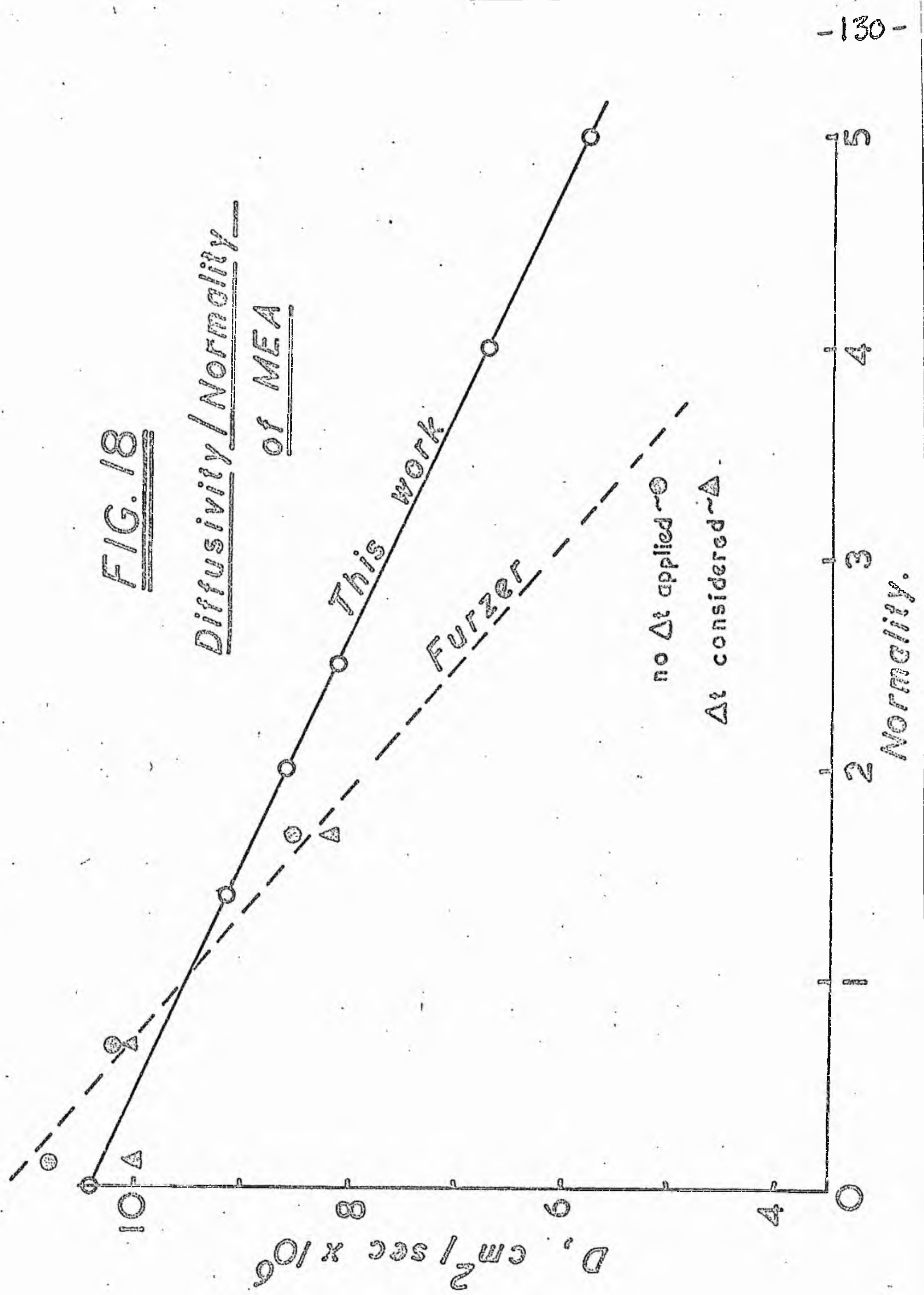
0

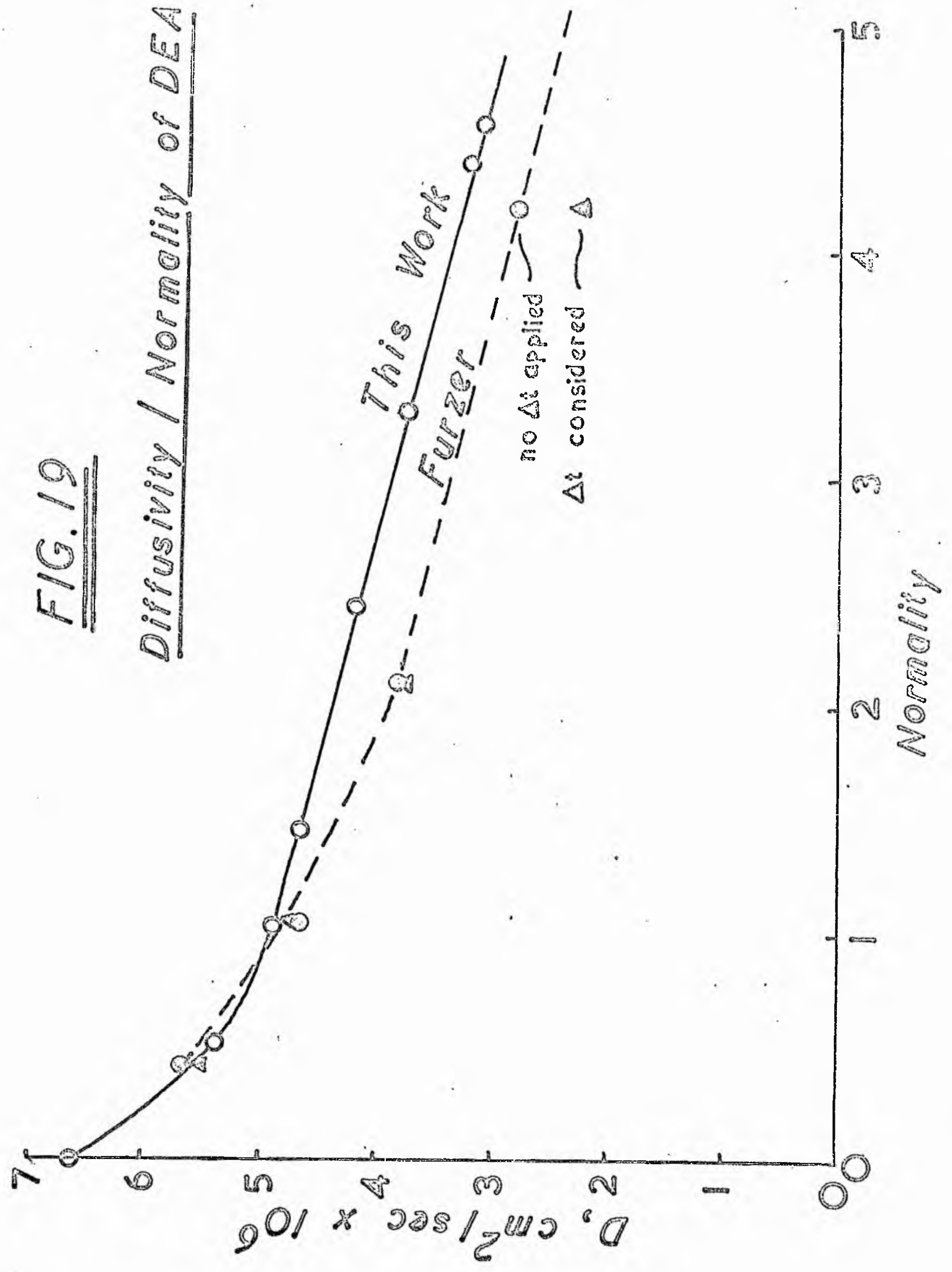




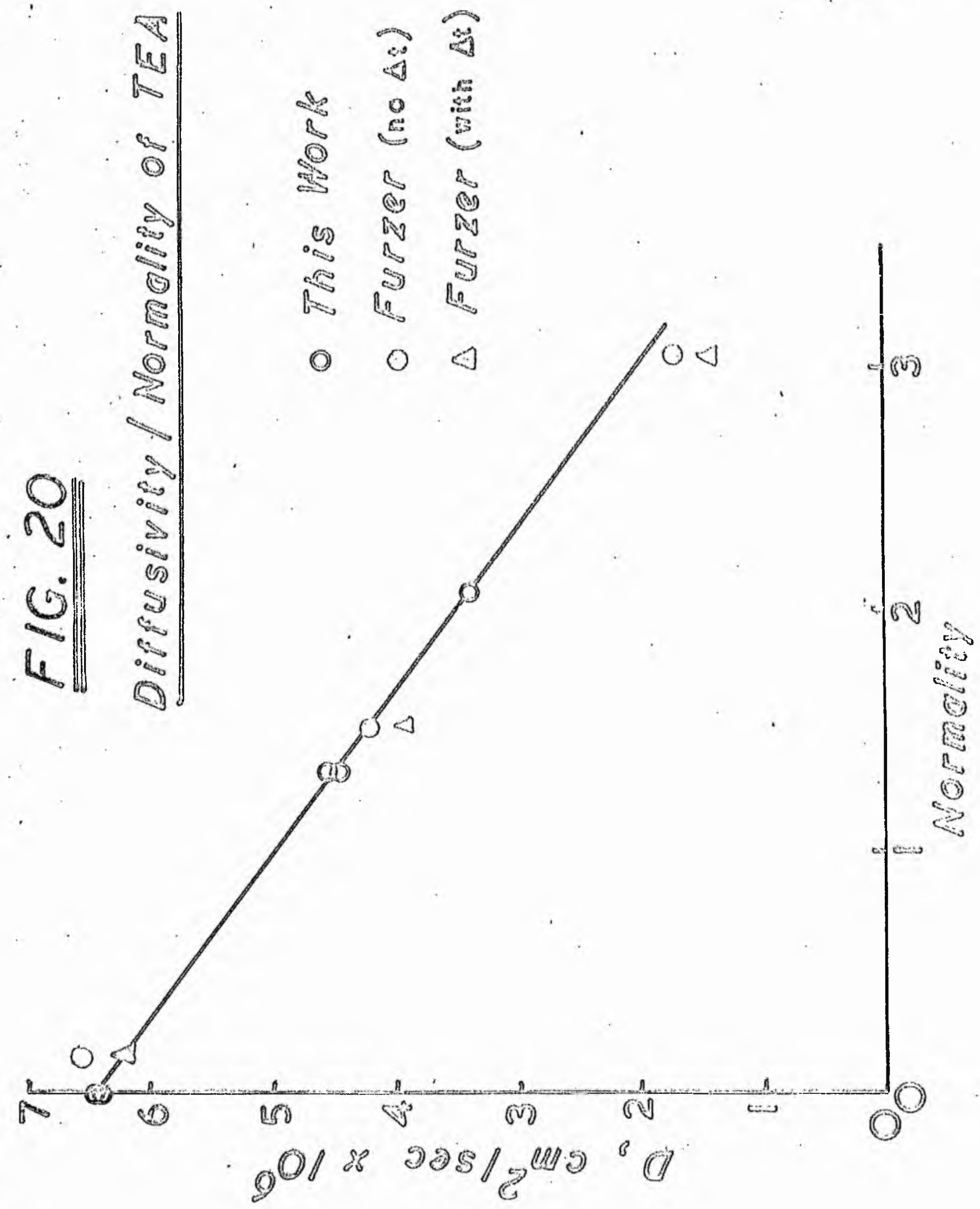


Conclusions from a Comparizon of Gouy and Wavefront Sheasing moterferometer Results.

When a At is applited to the Thomas and Furrei" results, a large variation is observed。 i.e. for TEA $1, A t=-42$ secs. "TEA 4 , 出 $=1339$ secs. There are also three other results (MEA 4, DEA 4, of 5 ) Where the $\Delta t$ is inordinatiely laxge o The implication of this is that there is either quite a substantial inherent inaccuracy in the Gour interferometer, or that Thomas and Furzer did not obtain a shanp interface before diffusion strarted. There is also the possibility that too many iringes were obtained ( the concentration difference between the solutions being too great), so that too much information

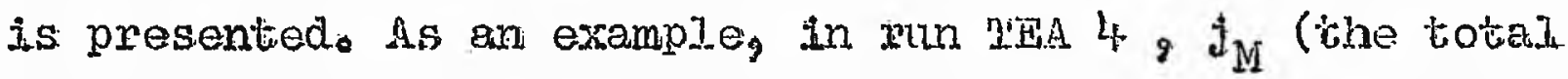
number of fringes) is of the order 75 -79, whexeas the ideal number of fringes should be less than 10. The inaccuracy factor of the Gouy results is further highlighted by an examination of $j_{M}$ values. IdeaII. $j_{M}$ valine should be constant throughout each experimentog but this does not appear to have been so. As an example, for the run TFA 4, the $j_{M}$ values calculated by Thomas and Fuszer 


\section{$\Rightarrow \mathrm{li} 3 \mathrm{j}_{1}=0$}

are tabulated below;

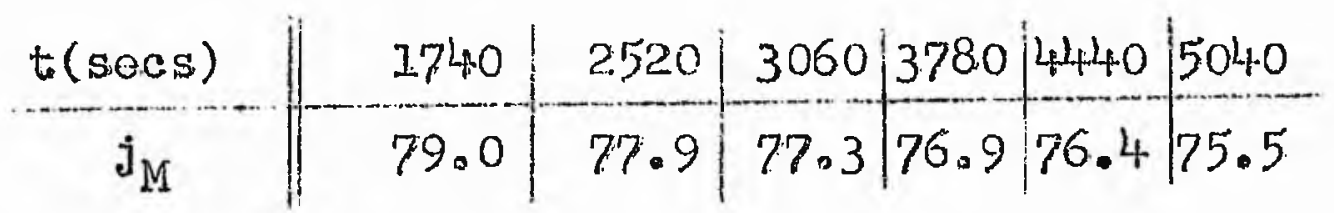

It is not proposed to discuss in detail how inaccuracy Is introduced into the results from the Gouy interferometer, suffice to say that. when compared with those: of the wavefront shearing interferometer, they appear to be considerably infexior. It should thus be taken that the results of this study are the more accurate.

From the graphs on which the comparisons are made, it would appear that although the Gouy results do differ, the shapes of the curves are approximately the: same. The Gouy resuits for monoethanolamine have one point ( at $3.34 \mathrm{~N}$ ) which is widely different, and this point effectively throws the whole curve out of any agreement with the wavefront shearing interferometer results.

One important observation to be made is that. the diffusivity values at. Iow concentrations are very similar. It was these D-vailues on which Ibrahim and Kuloor were most critical. Since Thomas and Furzer's results are almost the same as those in this study, it would therefore appear that a radical renappraisal of the application of the semi- empirical equations to ethanolamine diffuston is called for. 


\section{THE APPLICAT ION OF THE SEMI-BMPIRICAL}

\section{BQUATIONS TO ETHANOLAMTNEWWATER DIFFUSION *}

The second critical implication of Ibrahim and KuIoor mentioned earlier, ioe. that the semi-empirical equations applied do not adequately describe the ethanolamine-water diffusional process, will now be examined in detail. As well as examining the semi-empirical equations, the Iimitw ations of the theories of diffusion will be assessed. Starting with the theories of diffusion, the following comments may be made; -

1.) The stokes-Einstein Equation (eqn.20\&22) is onl $\$$ applicable for solutes with a molecular weight of $>300$. For the ethanolamines, $\mathrm{M}_{\mathrm{MEA}}=6.1 .08 ; \mathrm{M}_{\mathrm{DEA}}=105.14 ; \mathrm{M}_{\mathrm{TEA}}=$ $=149.2$. Thus, there can be no correlation between the experimental results for the ethanolamines and this theory.

2) Arnold's Kinetic Theory is of little use for prodicting diffusivity in any system, since three empirical constaits are involved in Arnold's equation (eqm.36). Two of these constants axe known for common solvents ( 0. g. water a.t. $25^{\circ} \mathrm{C}$ ) but the third constant, relating to the solute, is somewhat arbitrary. The Arnold equation has been applied 
to the diftusion of ethanolamines in this study, with the intention of relating this third constant to the apparent molecular volume of the solute in solution.

3.) Eyxing's Theory is represented by equation 33, this equation being a simplification of equation 37. There are two Important drawbacks to the use of this theory; a) the inclusion of an empirical parameter describing the geometrical configuration of the solute/solvent molecular structure, and $b$ ) the assumption that the activation energies for viscosity and diffusion are equal. Reported results on the application of the Eyring theory to various systems showed wide deviations from the experimental results. In view of these considerations, no attempt has been made to correlate the Eyring theory with the experimental results for the ethanolamines.

Gainer and Metznex's improvement on the Eyring theory is of little use In this case. The parametric empirical constants are still present, but the main difficulty with Gainer and Metzner's treatment is that the heat of evaporation of a hydrocarbon homologue must be callculated: With the ethanolamines this calculation is not posisible.

4) Nemnsts Law (equation 51 ) is applicable to the diffusion of a strongly lonised electrolyte, where the lonic 


\section{$-737 \%$}

mobilities of the cation and anion are known. Since this information is not arailable for the ethanolamines, the equation cannot be applied in this case.

5) The Nernst onsager Fuoss theory is too complex for a study of the nature conducted in this laboratory. The theory is only of use in a detailed physicomchemical study.

Thus, for the systems ethanolaminesmwater the pred.. iction of diffusivity is Iimited to the semi-empirical equationso Bearing in mind the objections ralsed to these equations ( as discussed previously ), - one primary demerit being the failure to allow for molecular irregularities, it Is not surprising that comparatively large deviations between prediction and observation will occur:

The four semimempirical equations that will be examIned relative to the ethanolamine diffusivity measurementis
are; -
a) Wilke-chang.
b) Scheibel.
c) Othmer-Thakar.
d) Ibrahim-KuIoor.

The molecular volumes, calculated from the atomic volumes due to Le Bas, are; - 


$$
\begin{aligned}
\text { i) } \mathrm{MEA}, \mathrm{V}_{\mathrm{A}} & =73.4 \\
\text { ii) } \mathrm{DEA}, & \mathrm{V}_{\mathrm{A}}=126.7 \\
\text { iii) } \mathrm{IEA}, & \mathrm{V}_{\mathrm{A}}=180.0
\end{aligned}
$$

and these molecular volumes will be used in all calculations relating to the semi-empirical equations.

( As an example of the calculation of $\mathrm{V}_{\mathrm{A}}$, consider diethanolamine, $-\mathrm{NH}\left(\mathrm{CH}_{2} \mathrm{CH}_{2} \mathrm{OH}\right)_{2}$

$$
\begin{aligned}
4 \times C & =4 \times 14.8=59.2 \\
I I \times H & =11 \times 3.7=40.7 \\
2 \times 0 & =2 \times 7.4=14.8 \\
I \times N=1 \times 12.0 & =\frac{12.0}{126.7}
\end{aligned}
$$

Taking the above values for $V_{A}$, and substituting into the semi-empirical equations, the predicted diffusivities are obtained; these diffusivities are given in Table 5. Also tabulated are the calculated molecular volumes $\left(V_{A}^{3}\right)$ which would bring the predicted diffusivities into agreement with the observed values. On the assumption that the discrepancies between predicted and observed values are entirely due to hydration of the solute, a hydration factor, $\phi$, has been calulated . i.e. $\phi=\frac{V_{A}^{\prime}-V_{A}}{v_{\text {water }}}$ 


\section{$m 139$ m.}

The hydration factor is also tabulated. The assumption of hydration of the solute will be explained in the following section.

A comparison has also been made between the solute empirical constant necessary to satisfy Arnol d $\mathrm{d}$ equation if this is to give the observed diffusivityg and this is compared with the ratio $\mathrm{V}_{\mathrm{A} / \mathrm{V}_{\mathrm{A}}}^{\mathrm{s}}$

Sample calculations for monoethanolamine follow, and the complete calculations are summarised in table 5.

Specimen Calculation of the Application of the Semi-Fmpixical Equations to Dilute MEA-water.

$$
\begin{aligned}
& \text { a) Wilkemchang. } \quad D_{0}=7.4 \times 10^{-008}(x M)^{\frac{1}{2}} \cdot T \\
& \mu \cdot v_{\mathrm{A}}^{0.6} \\
& V_{A}^{0.6}=(73.4)^{0.6}=13.16 \\
& \mu=0.8897 \mathrm{cp} \\
& T=298^{\circ} \mathrm{K} \\
& (\mathrm{xM})^{\frac{1}{2}}=(2.6 \times 18.016)^{\frac{1}{2}}=6.85 \\
& \text { whence } D_{0}=12.89 \times 10^{-6} \mathrm{~cm}^{2} / \mathrm{sec} \text {. }
\end{aligned}
$$




\section{$-140$}

$$
\text { fori } \begin{aligned}
& D_{0}= 10.40 \times 10^{.06} \\
&\left(V_{A}^{\prime}\right)^{0.6}=12.89 / 1.0 .4 \times\left(V_{A}\right)^{0.6} \\
& \therefore V_{A}^{\prime}=105 . \\
& \text { and } \frac{V_{A}^{\prime} / V_{A}}{}=1.43 .
\end{aligned}
$$

b) Scheibel.

$$
D_{0}=8.2 \times 10^{-8} \cdot \frac{T}{\mu}\left[\frac{1+\left(\frac{3 V_{B}}{V_{A}}\right)^{2 / 3}}{V_{A}^{I / 3}}\right]
$$

$$
\begin{aligned}
\left(\frac{3 V_{B}}{V_{A}}\right)^{2 / 3} & =\left(\frac{3 \times 18.9}{73.04}\right)^{2 / 3}=0.9131 \\
V_{A}^{1 / 3} & =4.187 \\
\therefore D_{0} & =12.55 \times 10^{-6} \mathrm{~cm}^{2} / \mathrm{sec}
\end{aligned}
$$

Put $V_{A}^{\prime}=100, D_{0}=9.972 \times 10^{-6}$

Put $V_{A}^{\prime}=90, D_{0}=10.64 \quad "$

when $v_{A}^{\prime}=93.5, D_{0}=10.4 \times 10^{-6}$

$$
\therefore \quad \mathrm{V}_{\mathrm{A} / \mathrm{V}_{\mathrm{A}}}^{\prime}=1.27
$$


c) Othmer and Thakar.

$$
D_{0}=\frac{14.0 \times 10^{-5}}{\mu .1 .1 V_{A}^{0.6}}
$$

$$
\therefore D_{0}=\frac{1.40 \times 10^{0.5}}{(0.8895)^{1.2}(73.4)^{0.6}}=\underline{12.10 \times 10^{.06}}
$$

For $D_{0}=10.4 \times 10^{-66}, \quad\left(V_{A}^{3}\right)^{0.6}=\frac{12.1}{10.4}\left(V_{A}\right)^{0.6}$

$$
\therefore V_{A}^{\prime}=94 \text { and } \quad V_{A}^{\prime} V_{A}=1.28
$$

a) Arnold's Equation, $D_{0}=\frac{B_{0} \sqrt{\frac{1}{M}+\frac{1}{M_{2}}}}{A_{1} \cdot A_{2} \sqrt{\mu / 1}\left(v_{1}^{1 / 3}+V_{2}^{I / 3}\right)^{2}}$

For solvent water at $25^{\circ} \mathrm{C}, \mathrm{B}=0.0108$

$$
\begin{aligned}
& \therefore A_{2}=\frac{0.0108 / \frac{1}{\frac{1}{18}}+016+\frac{1}{61.08}}{\left(10.4 \times 10^{-66}\right)(4.7)(0.8897)^{\frac{1}{2}}\left(18.9^{1 / 3}+73.4^{1 / 3}\right)^{2}} \\
& \therefore A_{1}=1.338
\end{aligned}
$$




\section{$=342$}

\section{Table 5}

Summery of the SemimEmpirical Equations Calculatjons.

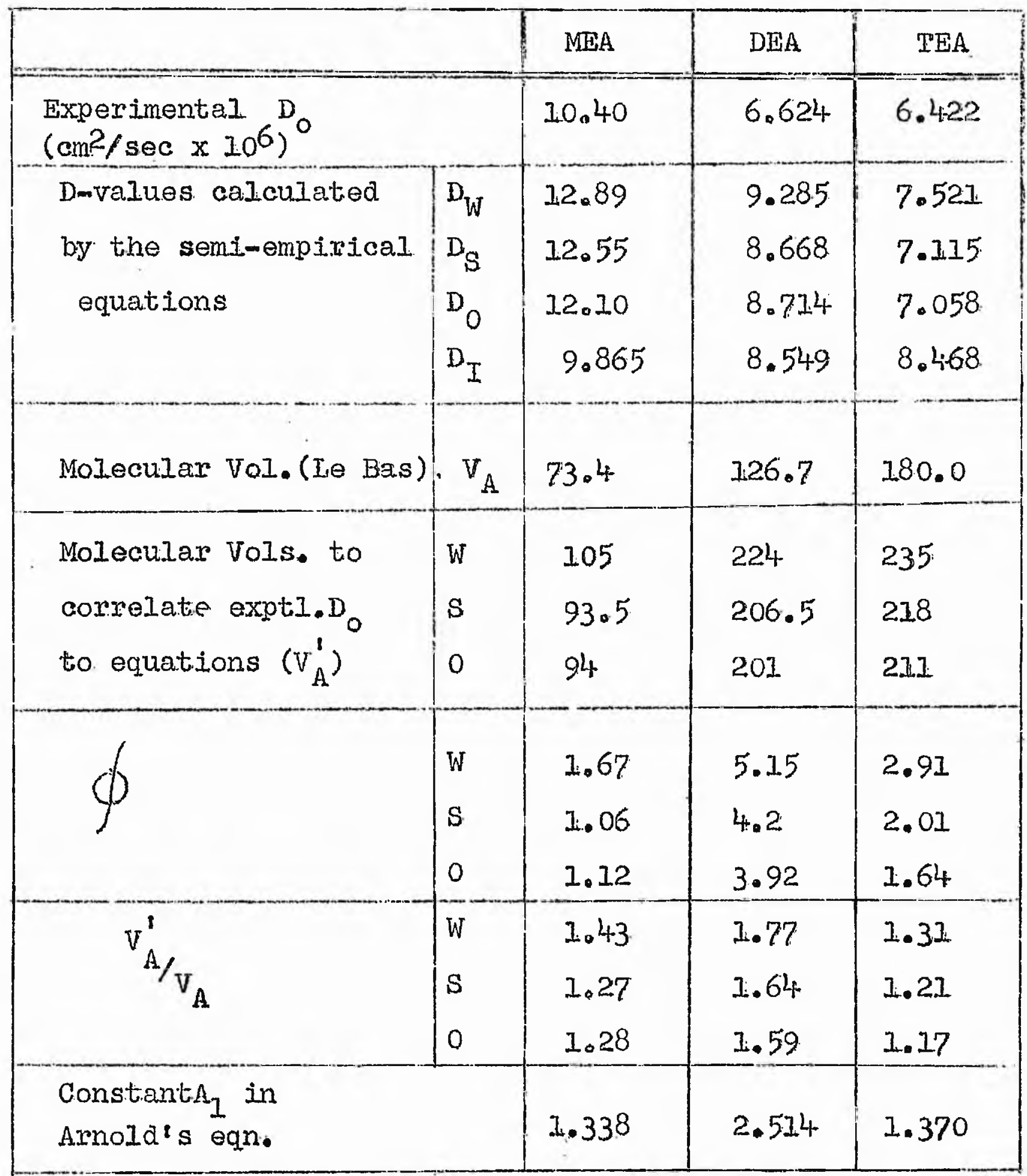


As may be seen from the calculations summarised in table 5, there is a significant discrepancy between the diffusion coefficients predicted by the semimempirical equations and the values obtained experimentally.In an attempt to correlate the agreement between the equations and the experimental. results, two viewpoints may be taken;...

i) The equations are not sufficient in themselves to predict diffusivity, ${ }_{2}$ assuming that $V_{A}$ and $V_{B}$ are correct.

ii) The value of $V_{A}$ as calculated from Le Bas is in error, due to molecular irregularities of the solute in solution.

The first viewpoint is substantiated by the section "...comments on the...theories and correlations", Ch.I(f), in which it was pointed out that none of the semimempirical equations could be relied upon to predict a D-value to much betiter than $10 \%$ a Some substantial deviations were illustrated.

However, one system serves to 1 llustrate the generaliy poor level of prediction of these equations, - the system being sucrose-water. Sucrose-water is a standard system for diffusion measurement calibrations, the value of $D$ being: confirmed as $D_{0}=5.22 \times 10^{-6} \mathrm{~cm}^{2} / \mathrm{sec}$ at $25^{\circ} \mathrm{C}$ 。 In solution, though; sucrose forms a hydrate. 
Scatchard ( 62 ) reviews previous determinations of the hydratiton of sucrose, and suplements them with his own accurate ineasurements from the vapoun pzessume of sucrose solutions. Fitem these, the average degree of hydration of sucrose in vexy dilute solutions was found to be 6 ( $t_{0} e_{0}$ Sucrose $\left.=\mathrm{G}_{12} \mathrm{H}_{22} \mathrm{O}_{11}{ } 6 \mathrm{H}_{2} \mathrm{O}\right)$, Taking the molecular volume of anhydrous sucrose as $\mathrm{V}_{\mathrm{A}}=340.4$ and the molecular volume of the hexahydrate as $v_{A}^{2}=4.53 .8$, the following diffusion coefficients in dilute solutions were predicted. by the semi-empirical equations;

Wi.like-Chang

Scheibel

othmer-Thakar

\begin{tabular}{l|c|c} 
& $\mathrm{C}_{12} \mathrm{H}_{22} \mathrm{O}_{11}$ & $\mathrm{C}_{12} \mathrm{H}_{22} \mathrm{O}_{11} \cdot 6 \mathrm{H}_{2} \mathrm{O}$ \\
\hline Wi.lke-Chang & 5.131 & 4.379 \\
Sche1bel & 5.124 & 4.467 \\
Othmer-Thakar & 4.816 & 4.054
\end{tabular}

(all the above Dmvalues in $\mathrm{cm}^{2} / \mathrm{sec} \times 10^{6}$ )

If Arnold's equation is applied ( using $V_{A}=340,4$ ), and putting $D_{0}=5.22 \times 10^{\circ .5}$,

$$
A_{I}=1.29
$$

It is interesting to note that if the anhydrous molecular volume of 340.4 is multiplied by a factor of 1.29 ,

$$
V_{A}^{\prime}=440
$$


This is not very different from the calculated molecular volume for the hexahydrat: $\theta_{\text {。 }}$

The exrors of prediction of the equations for: the sucrose-water system are,
a) $V_{A}=340.4$
1. $5 \%$
$1.75 \%$
; $7.5 \%$
b) $V_{A}^{1}=453.8$
$17 . \% ; \quad 15 \%$
; $22 \%$

It is thus seen that the semi-empirical equations are not really sufficient in themselves in the prediction of diffusivity. The first viewpoint taken above, therefore, is justified to a large extent. With the ethanolamines, however, the assumption that the inaccuracy of prediction is increased ( over the inaccuracy due to the insufficiency of the equations) by the wrong determination of $V_{A}$ is also vaild.

In the following section it will be shown that the ethanolamines are irregular in soiution. This irregularity. is cleaxly evident from several considexations, but attempts at calculating the degree of irregularity were noi wholly successful. The simplest explanation is to assume the ethanolamines to be hydrated in solution, and to calculate an approximate hydration number in an attempt to correlate the experimental results with the predictions a little more successfully. 
V. THE PHYSTCAI CHEMISTRY OF THE REAANOLAMINES

INY SOLUTION.

Ibrahim and Kuloor ( 3 ) made the doubtrul and unsupported statement that " all the physical propexties of the ethanolamines, except freezing point and specific heat, show a simple gradation from mono.. to tri-.." This statement was made in an attempt to say that diethanolamine should not be much more irregular in solution than eithes mono- or triethanolamine; even though Thomas and Furzer's, results $(1)(2)$ had first indicated this to be so.

From table 6, an examination of the tabulated physical properties of the pure amines (i.e. not in solution) shows that there is an apparent simple gradation in all properties apart from the freezing pointo (note that, contraxy to Ibrahim and Kuloor, the specific heat DOES show a simple gradation). The first indication of irregularity is apparent, however, when curves of the vapour pressures of pure amines against temperature are compared. These curves are shown in Fig.21. Below a temperature of $130^{\circ} \mathrm{C}, \mathrm{DEA}$ has a lower total vapour pressure than TEA, whereas above this point the reverse is so. 


\section{$-14.7-$}

Table 6

Propexties of Pure Fithanolamines $(99.9 \%+) .(63)$

\begin{tabular}{|c|c|c|c|}
\hline & $\mathrm{MEA}$ & DEA & TEA \\
\hline MoI.Wt. & 61.08 & 105.14 & 149.2 \\
\hline Boiling pt., ${ }^{\circ} \mathrm{C}$ & 170.3 & 268.4 & 338.8 \\
\hline Freezing pto, ${ }^{\circ} \mathrm{C} \quad \leftrightarrow$ & 10.31 & 27.95 & 21.57 \\
\hline Density, $g \mathrm{~m} / \mathrm{mI}$ at $40^{\circ} \mathrm{C}$ & 0.9989 & 1.0828 & 3.1 .1 .16 \\
\hline Viscosity, $\mathrm{cps}_{9}$ att $40^{\circ} \mathrm{C}$ & 10.06 & 296.4 & 208.1 \\
\hline Refractive index, $n_{D}^{40}$ & 1.4474 & 1.47720 & 1.4798 \\
\hline Specific heat, $\left.30^{\circ} \mathrm{C}_{g}{ }^{\mathrm{ca}}\right)^{\mathrm{g}} \mathrm{gm}$ & 0.561 & 0.531 & 0.497 \\
\hline Flash point, ${ }^{\circ} F$ & 200 & 295 & 380 \\
\hline Heat of fusion, ${ }^{\mathrm{cal}} / \mathrm{mol}^{2}$ & 4900 & 6000 & 6500 \\
\hline Heat: of vap. , cal $/ \mathrm{mol}^{2}$ & $12,1,150$ & 15,590 & 16,127 \\
\hline
\end{tabular}

* triethanolamine tends to supercool. 


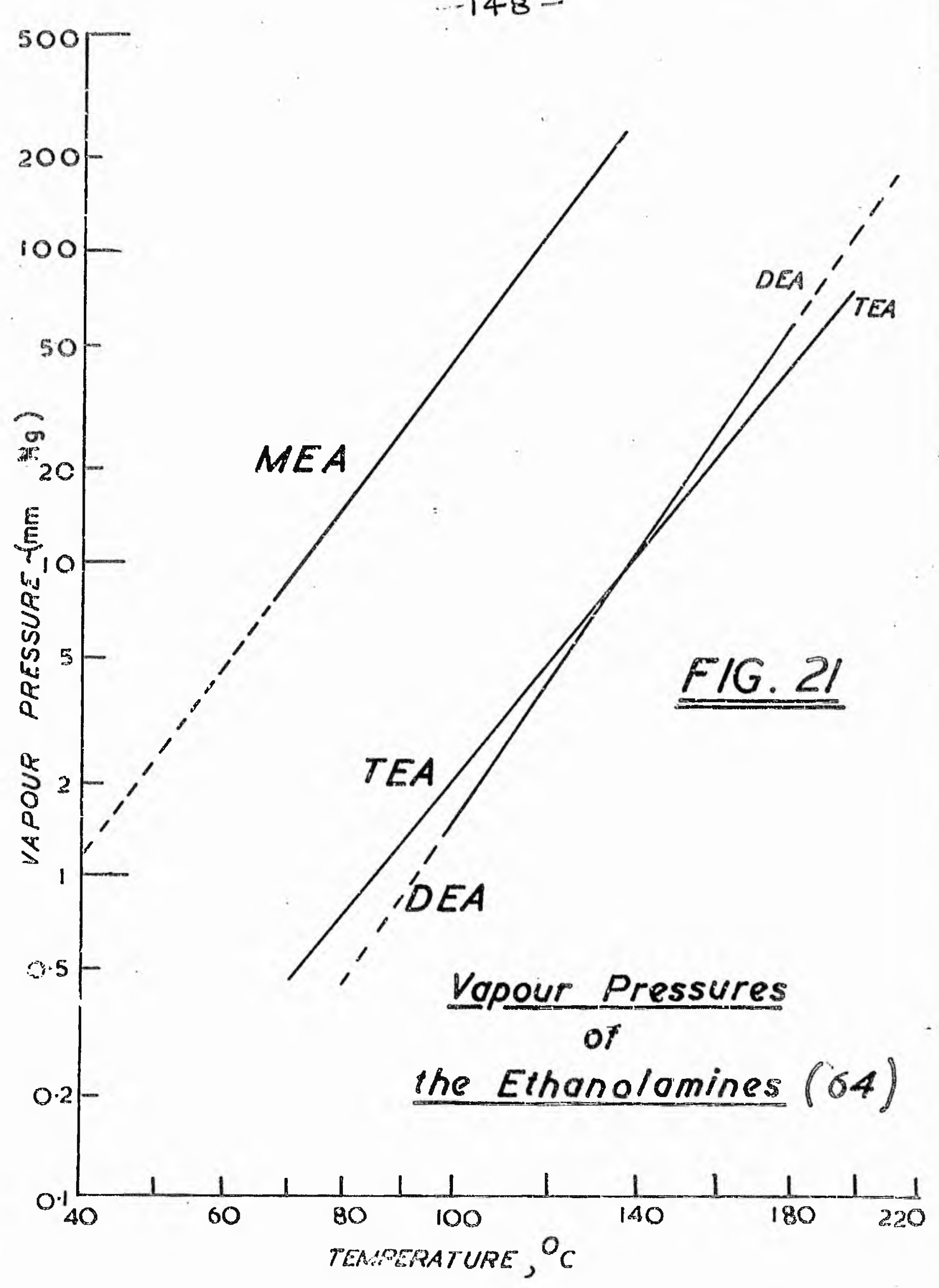


In solution, howeven, there is abundant evidence of irregularity in physical properties。 Thus Ibrahim and Kuloor should have considered the properties in solution rather than the properties or pure amines. The simplest demonstration of this irregularity is shown by viscosity measurements of the amines in solution, where DEA shows a markedly anomalous behaviour. Viscosity measurements were made in this laboratory of MEA, DEA, anf TEA solutions at $25^{\circ} \mathrm{C}$. These: measurements have been amalgamatted with the data of Leibush and Shneerson ( 65 ), the Shell Chemjcal $\mathrm{Co}_{\mathrm{n}}(64$ ) and Dow ( 63 ). The viscosities of amine soluitions at $25^{\circ} \mathrm{C}$ are tabulated in table 7, and the curves are shown in Fig.22.

A further indication of the anomalous behaviour of DEA, is the variation of the product $n_{0} \mu$ against concentration. Values for $\mathrm{D}_{0} \mu$ for $\mathrm{MEA}_{2} \mathrm{DEA}_{9}$ and TEA are tabulated in table 8 , and the curves of D. $\mu$ against concentration are shown in Fig. 23. 
$-150-$ 


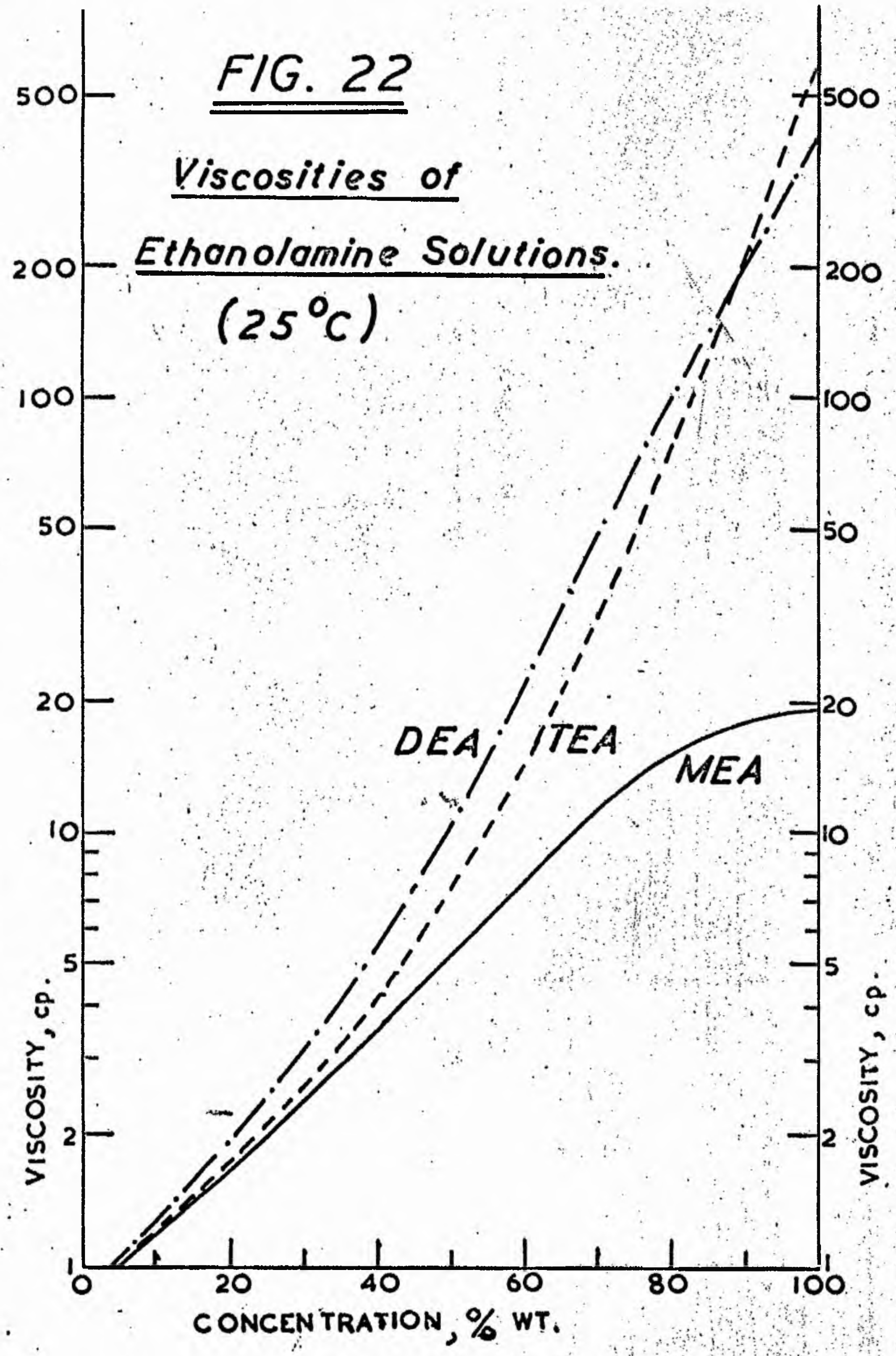


Tabile 7

Viscosities of Ethanolamine Solutions at $25^{\circ} \mathrm{C}(\mathrm{cps})$.

\begin{tabular}{|c|c|c|c|}
\hline $\begin{array}{c}\text { Conen. } \\
\text { Wt.\% }\end{array}$ & MEA & DEA & TEA \\
\hline 0 & 0.8897 & 0.88 .97 & 0.8897 \\
10 & 1.17 & 1.28 & 1.23 \\
20 & 1.66 & 1.93 & 1.75 \\
30 & 2.42 & 3.10 & 2.58 \\
40 & 3.52 & 5.47 & 4.08 \\
50 & 5.23 & 10.2 & 7.09 \\
60 & 7.55 & 22.4 & 14.28 \\
70 & 11.08 & 49.2 & 30.7 \\
75 & 13.4 & 69.8 & 49.2 \\
80 & 15.1 & 98.9 & 79.1 \\
90 & 17.4 & 199 & 206 \\
100 & 18.9 & 395 & 61.4 \\
\hline
\end{tabular}


$-152-$ 


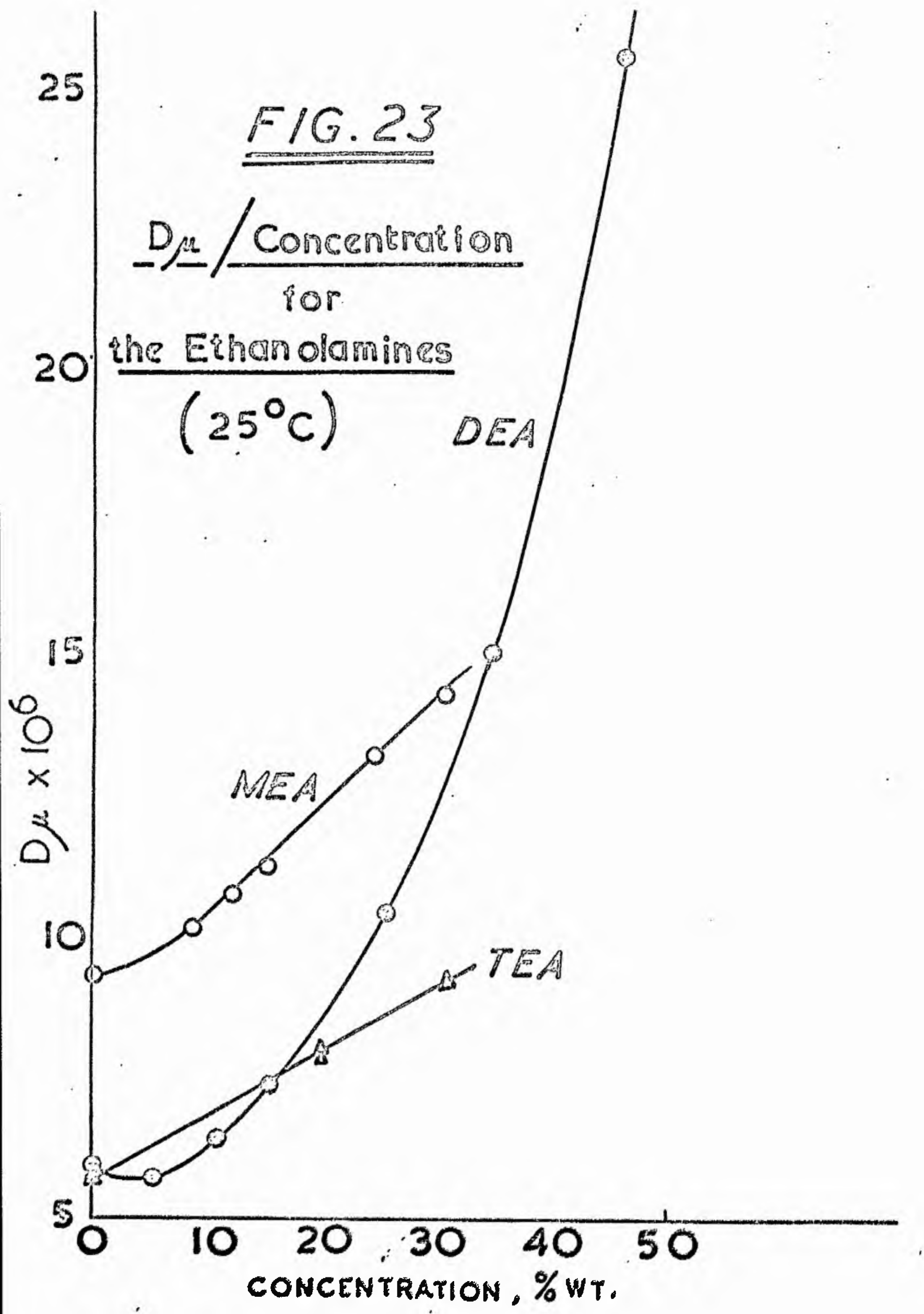


Tabile 8.

The Product D. fus for the Eiharolamines at $25^{\circ} \mathrm{C}$.

\begin{tabular}{|c|c|c|c|c|}
\hline & $\begin{array}{c}\text { Conch. } \\
\text { wt.\% }\end{array}$ & $\mathrm{cm}^{2} / \mathrm{sec} \times 10^{6}$ & $\frac{d h}{\cos }$ & $\begin{array}{l}D_{0}^{11} \\
\times 10^{6}\end{array}$ \\
\hline \multirow{6}{*}{$\mathrm{MEA}$} & 0 & 10.40 & 0.8897 & 9.26 \\
\hline & 8.6 & 9.116 & 1.110 & 10.12 \\
\hline & 7.2 .22 & 8.575 & 1.252 & 10.72 \\
\hline & 15.19 & 8.101 & 1.388 & 1.1 .23 \\
\hline & 24.45 & 6.729 & 1.960 & 13.18 \\
\hline & 30.56 & 5.770 & 2.470 & 14.24 \\
\hline \multirow{8}{*}{ DEA } & 0 & 6.624 & 0.8897 & $5: 89$ \\
\hline & 5.45 & 5.377 & 1.073 & 5.77 \\
\hline & 10.90 & 4.877 & 1.323 & 6.45 \\
\hline & 15.45 & 4.635 & 1.59 .1 & $7 \cdot 37$ \\
\hline & 25.6 & 4.181 & $2.4 \cdot 9$ & 10.4 \\
\hline & 34.72 & 3.733 & 4.02 & 1.4 .98 \\
\hline & 46.25 & 3.185 & 8.00 & 25.5 \\
\hline & 48.09 & 3.089 & 8.95 & 27.7 \\
\hline \multirow{5}{*}{ TEA } & 0 & 5.422 & 0.8897 & 5.71 \\
\hline & 0 & 6.446 & 0.8897 & 5.74 \\
\hline & 20.06 & 4.4 .52 & 3.75 & 7.80 \\
\hline & 20.06 & 4.564 & 1.75 & 7.98 \\
\hline & 30.9 & $3 \cdot 385$ & 2.715 & 9.19 \\
\hline
\end{tabular}


From the viscosity data for ethanolamine solutions at $25^{\circ} \mathrm{C}$, it can be seen that in aqueous solutions below about $90 \%$ (by weight) DEA has a higher viscosity than TEA。

From the curves of the product $D_{0} \mu$ against concentration, the DEA curve is very steep compared to the MEA and TEA curves, -which are approximately paraliel.

The above considerations indicate that aqueous diethanolamine solutions are anomalous in behaviours, and that therefore there is some form of association of diethanolamine in solution. Thus, if there is a hydration effect with all three amines, DES will tend to exhibtt greater irregularities in other physico-chemical measuroments than WiII MEA and TISA.

Trotman-Dickenson ( 66 ) made a qualitiative study of the Importance of hydration effects on the basic strength of amines. Condon ( 67 ) presented a reneral theory for the influence of hydration on the base strength of arntnes. Both these workers, ${ }_{2}$ however, limited their remarks to mainiy qualitative assessments of the structure of the amines in solution. Furthermore, the ethanolamines have not been included in their work, due to the presence of additional hydrophilice groups. It is clearly evident, however, from the work of Trotman-Dickenson and Condon that the ethanolaminas 
will be hydrated in solution, and to quite a significant effect.

For "regular" amines the hydration by hydrogen bonding is schematically shown below, 1. $\theta$.
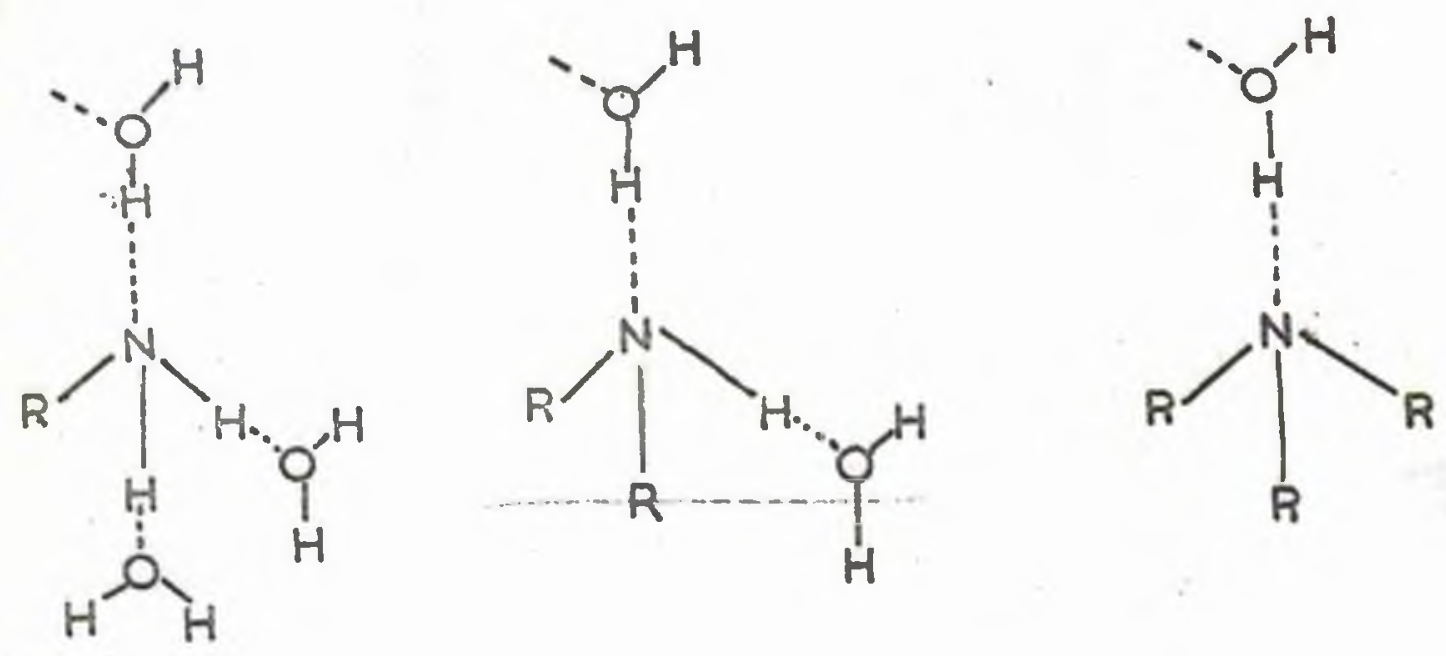

However, as mentioned above, the ethanolamines contain hydrophilic groups ( the $\mathrm{C}_{2} \mathrm{H}_{4} \mathrm{OH}$ groups). Thus as well as the hydration illustrated above, the hydration due to the presence of the alkanol groups must also be considered.

Although it is qualitatively certain that the ethanolamines are hydrated in solution, the extent of hydration is by no means clear. Correspondence with 
Professor Condon in fact established that the present state of knowledge on the hydration of amines is wholly inferential, - that is, based on the effect it produceso on considering Trotmanmickenson and Condon's work, therefore, the only conclusion to be drawn is that the ethanolanines are hydrated in solution, and with appreciabie effect. The deviation of the experimental aiffusivities of the ethanolamines from predicted values is explainabie on a basis that the molecular volumes concemed are effectively increased by the hydration effect. The greatest difference in values, shown by diethanolamine, is understandable on consideration of the irregularities shown bEA in solution (e.g. viscosity). All this is in addition to the conclusion that the semi-empirical equations are unsatisfactory in any case.

An attempt was made, throigh calculations based on hygroscopicity, refractive index measurements, and vapour pressure data, to estimate the hydration effect cuantitatively. The calculations nere made on the basis of a hydration number, of as defined previously, to estrimate the increase in $V_{A}$. These calculations are summerised in the next section. 
I(a). Estimation of Hydrate Foxmation in Ethanolamines from Hygroscopicitsy Measurements.

All three ethanolamines are markedly hygroscopic. $\mathrm{If}_{2}$ then, the asymptotic values of maximum hygroscopicity are taken from the curves cf Increase in weighto due to watex pick-up) against time, a pointer will be given as to of what order the maximum possible hydration will be. The data used is taken from shell ( 64). It should be noted that Shell recommend that the curves be onlyy used as approximate guides, since the results were affected by the greater volatility of monoethanolemine, and by the absorption of the ethanolamines by other specimens being tested at the same time.

Monoethanolamined Weight gain $=95 \%$ (1400 hrs.)

. LO0g MEA $\longrightarrow$ I00g MEA + 95g water (1.64 mols MEA + 5.25 mols water)

$$
\because \phi=3.2
$$

Diethanolamine. Weight gain $=68 \% \quad \therefore \phi=4$ Triethanoiamine. Weight gain=50\% $\quad \therefore \phi=4.2$ 
VI(b) Estimation of Hydration of Diethanolamine

from Refractive Index Measurements,

Refractivities of Solutions.

Use has been made of refractive index measurements of diethanolamine solutions to test: the variation of the experimental values from refractive indices predicted from theoretical considerations.

Partington ( 69 ) reviews the equations applicable to refractive indices in solution.

A simple formula. was proposed by Beer, Gladstone, and Dale (Phil.Trans. 148,88,1858) in which the refractivity of a gas or liquid over a temperature range is connected by the formulat

$$
\frac{n-1}{\rho}=\text { constant }=r_{G}
$$

A Iittle 1ater, Iorenz (Ann. Phys, 11,70, 1880) and Lorentz (Ann.Fhys. $2,641,1880$ ) proposed the most commoniy used refractivity formula;

$$
\frac{n^{2}-1}{\left(n^{2}+2\right) \cdot \rho}=\text { const. }=x_{I}
$$


where $r_{\mathrm{L}}$ and $r_{\mathrm{G}}=$ molar refractivities

$$
\begin{aligned}
& \mathrm{n}=\text { refractive index } \\
& \rho=\text { density }
\end{aligned}
$$

For liquid mixtures and solutions the refractivity is considered to be additively composed of proportions by weight. It has also been found by numerous workers that the Lorenz-Lorentz equation is more suitable for many systems. Thus, in molar terms, the following relationship between the refractivity of the solution and the refractivity of the components ( $I$ and 2 ) is derived;-

$$
\left.\left(\frac{M}{\rho}\right)_{\left[\frac{n^{2}-1}{n^{2}+2}\right.}\right]_{1,2}=x_{1}\left(\frac{M}{\rho}\right)_{1}\left[\frac{n^{2}-1}{n^{2}+2}\right]_{1}+x_{2}\left(\frac{M}{\rho}\right)_{2}\left[\frac{n^{2}-1}{n^{2}+2}\right]_{2}
$$

where $M=$ mol.wt., and $x=$ mol fraction.

The above equation is sometimes written,

$$
[R]_{1,2}=x_{1}[R]_{1}+x_{2}[R]_{2}
$$

The above equation has been employed to determine whether refractive index measurements can give any indication as to the molecular abnormality of diethanolamine solutions. Consider a solution made up of an ethanolamine, subscript $\mathrm{E}$, and water, subscript W. Assuming no hydration, then if wh. of ethanolamine $=\mathrm{w}_{\mathrm{E}}$ and wt. of water $\quad=w_{W}$ 


$$
\begin{aligned}
\text { then, mols ethanolamine }=\mathrm{W}_{\mathrm{E} / \mathrm{M}_{\mathrm{E}}} & =\mathrm{x}_{\mathrm{E}} \\
\text { mols water }=\mathrm{W}_{\mathrm{W}} / 1.8 .016 & =x_{\mathrm{W}}
\end{aligned}
$$

However, if $\mathrm{z}$ mols water combine with $\mathrm{x}_{\mathrm{E}}$ mols ethanolamine, the following equation is obtained by substituting into the Lorenz-Iorentiz equation;

$$
\begin{aligned}
{[R]_{1,2}=} & {\left[\frac{x_{E}+z}{x_{E}+x_{W}}\right]\left[\frac{M_{E}+\frac{z}{x_{E}} \cdot M_{W}}{\rho_{E}}\right] x_{E} } \\
& +\left[\frac{x_{W}-z}{x_{F}+x_{W}}\right]\left[\frac{18.016}{W}\right] x_{W} \quad \ldots .(99)
\end{aligned}
$$

The main assumption that has been taken above is that the density of hydrated amine solution is the same as that for pure amine.

Solving for $\mathrm{Z}$ in the above a quadratic is obtained:

$$
\begin{gathered}
{\left[\frac{18.016 . x_{E}}{x_{E} \cdot \rho_{E}}\right] z^{2}+\left[\frac{r_{E} M_{E}}{\rho_{E}}+\frac{x_{E} \cdot 18.016}{\rho_{E}}-x_{W \cdot 18.016}\right] z} \\
+\left[\frac{x_{E} x_{E} M_{E}}{\rho_{E}}+x_{W} W_{W}-R_{I, 2}\left(x_{E}+x_{W}\right)\right]=0
\end{gathered}
$$

and from $z, \phi$ may be found. 
The Refractive Index of DEA Solutions.

The refractive Index of 12 diethanolamine solutions was measured at $25^{\circ} \mathrm{C}$, using a Pulfrich refractometer. A brief description and explanation of the method is given.

In the Pulfrich refractometer, the liquid under test is put in a small quartz cell in contact with a prism. Monochromatic light from a low pressure sodium lamp is focussed through the cell and the angle of refraction is measured by rotating the refractometer telescope and centerring the cross-wires on the emergent sodium D-line. 1. 8 .

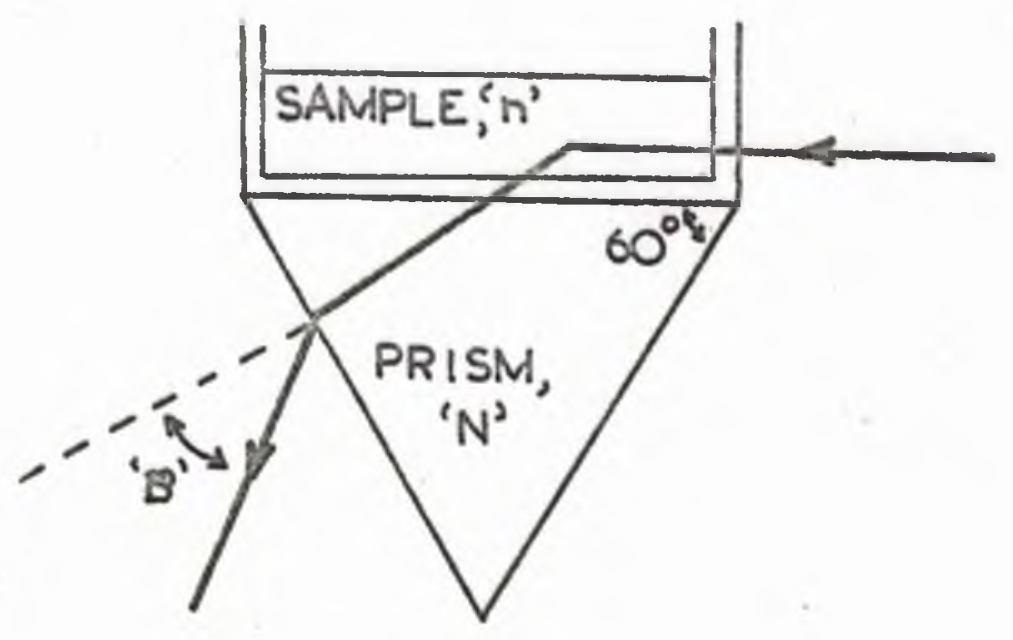


For a $60^{\circ}$ prism the general equation applicable

is

$$
n=\sin 60^{\circ} \sqrt{N^{2}-\sin ^{2} B}-\cos 60^{\circ} \sin B \ldots \text { (IOI) }
$$

where $\mathrm{n}=$ refractive index of the sample

iN $=$ refractive index of the prism

$L_{B}=$ angle of emergence(with respect to the nomal)

$N$ is determined by measuring $B$ with the coll empty. Substituting $n=I$ ( refractive index of air) in the general equation, and putting $\angle \phi=4 B$,

$$
\mathbb{N}^{2}-I=\left[\frac{\sin \phi+\cos 60^{\circ}}{\sin 60^{\circ}}\right]^{2}
$$

Using the Pulfrich, the refractive index of a sample may be measured to an accuracy of $\$ 0.00002$.

AlI measurements were made at a constant temperature of $25^{\circ} \mathrm{C}$, the cell and prism being jacketed by a water bath. of the 12 solutions tested, two are in obvious exror when the refractivities are plotted against concentration. These are $9.5 \%$ and $47 \% \mathrm{DEA}$. All the other points are accurate. The refxactivity measurements are given in tabile 9.

Using the experimental results and also refractivity values published by Shell( 64), the possible degree 


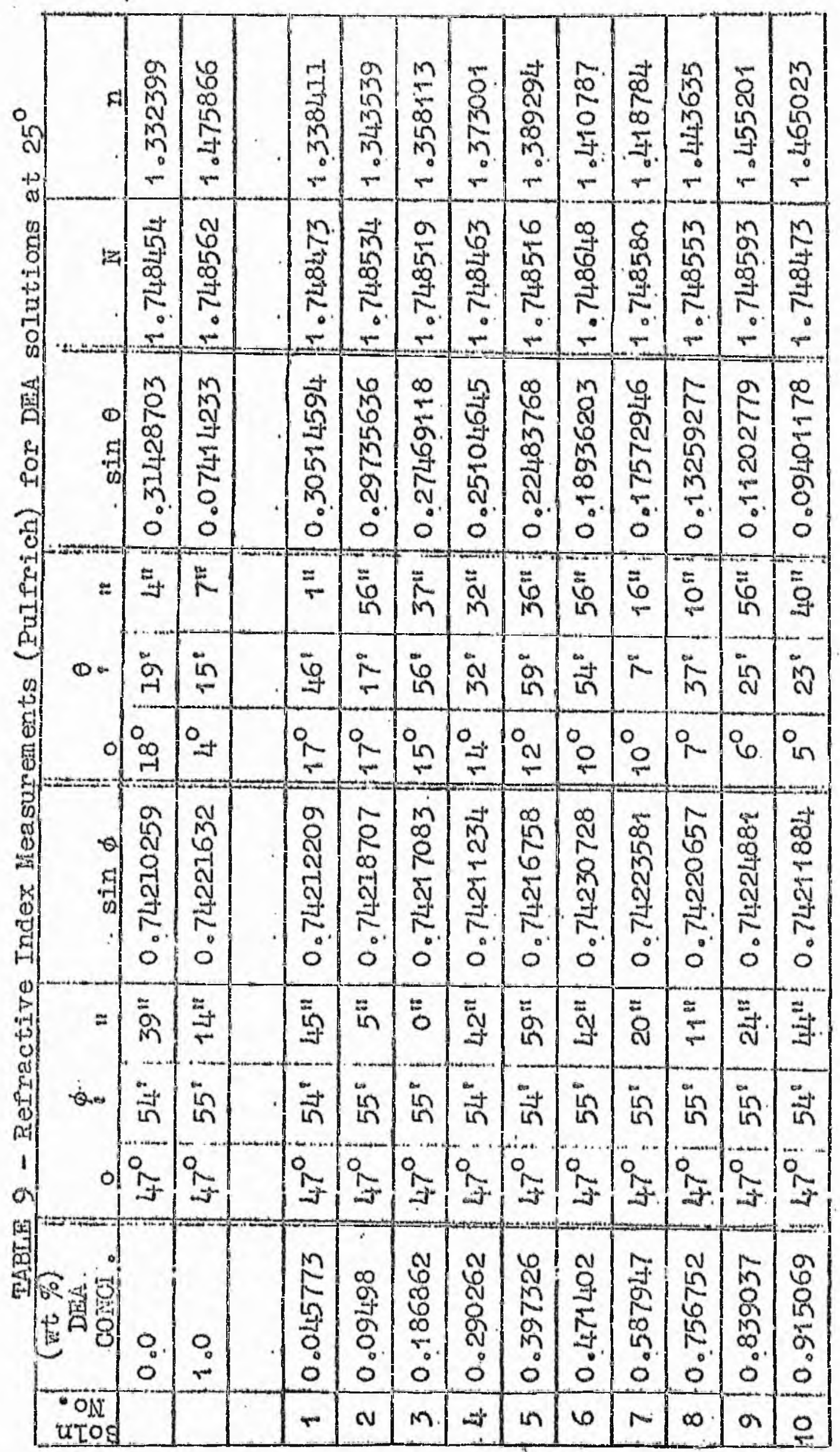


of hydration was calculated as above. The theoretical refractive index of the solutions (assuming no hydration) was also calculated. The results are shown in table 10.

\section{Table 1.0}

Calculation of DEA Hydration from refractivity Data.

\begin{tabular}{|c|c|c|c|c|}
\hline $\begin{array}{c}\text { DEA Concn. } \\
\text { wt. } \%\end{array}$ & $\mathfrak{n}_{\exp }$ & $n_{\text {theor. }}$ & $n_{\exp }{ }^{-n}$ th. & $\rho$ \\
\hline \multicolumn{5}{|c|}{ DEA Solutions at $25^{\circ} \mathrm{C}$ (experimental) } \\
\hline 4.58 & 1.33841 & 1.33827 & 0.00014 & 0.05 \\
\hline 18.69 & 1.3581 .1 & 1.35675 & 0.00136 & 0.02 \\
\hline 29.03 & 1.37300 & 1.37020 & 0.00280 & 0.02 \\
\hline 39.73 & I. 38929 & 1.38545 & 0.00384 & 0.02 \\
\hline 58.79 & 1.4 .1878 & 1.41267 & 0.00611 & 0.02 \\
\hline 75.68 & $1.44+364 \mathrm{~F}$ & 1.43781 & 0.00583 & 0.01 \\
\hline 83.90 & 1.45520 & 1.45044 & 0.00476 & 0.01 \\
\hline 91.51 & 1.46502 & 1.46233 & 0.00269 & 0.01 \\
\hline \multicolumn{5}{|c|}{ DEA solutions at $60^{\circ} \mathrm{C}$ ( Shell) } \\
\hline 25 & 1.36380 & 1.35958 & 0.00422 & 0.07 \\
\hline 50 & 1.39850 & 1.39315 & 0.00535 & 0.03 \\
\hline 75 & 2.43500 & 1.42864 & 0.00636 & 0.02 \\
\hline 90 & $1.454+70$ & 1.45092 & 0.00378 & 0.01 \\
\hline 95 & 1.46050 & 1.45851 & 0.00199 & 0.00 \\
\hline
\end{tabular}


Comments on the Refractive Indices of DEA.

In both the Shell data and the experimental results the difference between the experimental and the calculated refractive indices is appreciable ( maximum of 0.0064 in 1.435 ). However, calculations to determine the possible hydration using the Lorenz-Lorentz equation showed insignificant changes in the constitution of the ethanolamine ( maximum possible hydration of 0.07 mols water to 1 mol DEA).

Further calculations were made to see what effect an appreciable degree of hydration should have on the experimental refractive index if the Lorenz-Iorentz equation was strictly true. ( $1 . e$ the molecularity of the solution components being of relevance).

Using the figures for $25 \% \mathrm{DEA}$ at $60^{\circ} \mathrm{C}$ (these indicating the greatest molecular deviation) the refractive index that would be observed if the equation were true was calculated for two cases;

Ascuming 1) 0.25 mols water to 0.2377782 mols DEA $(\phi=1.05)$ 2) 0.5 " " " " $" \phi=2.1)$ (since 25\% $\mathrm{DEA}=0.237782 \mathrm{mols} \mathrm{DEA}_{2} 4.1629662 \mathrm{mols} \mathrm{H}_{2} \mathrm{O}$ ) The refractive indices that would satisfy the equation were 


\section{$\sin 6$.}

calculated to be I) $1.51858 \quad \therefore \Delta_{\text {ex. }}{ }^{-n_{t h}}=0.15900$

2) $1.75814: n_{e x{ }^{-n} n_{t h}}=0.39856$

$n_{\text {ex. }}-n_{\text {th. }}$ beIng 40 and 90 times greater respectively than the observed values.

Calculations were then made to see whether an assocjated molecule would be indicated by the experinental refractive index. Again, for the $25 \%$ solution, the increased molecular weight that would account for the deviation was: insignificant, - being 108.9 ( $\mathrm{M}_{\mathrm{DEA}}=105.14$ ).

It is apparent, then, that the measurement of the refractive indices of a range of solutions is not a valid method of determining molecular irregularity in solution. This could be due to several reasons, such as the inaccuracy of the LorenzmLorentiz equation. One factor that seems very probable is that the refractive index is an additive function of the pure components only. Thus, hydxation and association, which are physical and not chemical changes, would not influence the refractive index to any great extent. i.ed if we have $\mathrm{X}$ mols DEA, $\mathrm{Y}$ mols $\mathrm{H}_{2} \mathrm{O}$, and the DEA hydrates with $z$ mols of this water, then the refractive index is not influenced by this movement of $\mathrm{Z}$ to $\mathrm{X}$ at. ail,-being $\mathrm{a}$ function only of $\mathrm{X}$ and $\mathrm{Y}$. 
$V I(c)$ Estimation of the possible Hydration of the Pthanolamines from Vapour Pressure Data.

The total vapour pressure of a binaxy solution is related to the mol. Praction of its components by the expression,

$$
x P_{1}+(1-x) P_{2}=\pi
$$

where $P_{I}$ is the partial pressure of component 1 , of concentration $x$ mol fraction; $P_{2}$ is the partial pressure of component 2 , of concentration $(1-x)$ mol fraction; and $\pi$ is the total pressure.

The total vapour pressures of 10\% MEA, 10\% DEA, and 20\% DEA solutions are taken from the data of the Shell Chemical Co. ( 70 ). The total vapour pressures (TT) are tabulated in table 11 , together with the vapour pressures of water $\left(P_{W}\right)$ and ethanolamine $\left(P_{E}\right)$. The vapour pressure of water is taken from Perry ( 71 ) and the vapour pressures of the ethanolamines are calculated from the data of MoDonald et al ( 72 ) : The Aitoine coefficients to satisfy the relationshlp $\quad \log _{10} \mathrm{P}_{\mathrm{E}}=\mathrm{A}-\frac{\mathrm{B}}{\mathrm{t}+\mathrm{C}}$

$$
\text { ares } \left.\left.\quad \begin{array}{l}
A=7.738 \\
B=1732.11 \\
C=186.215
\end{array}\right\} \quad \begin{array}{l}
\mathrm{MEA} \\
\mathrm{B}=8.13968 \\
\mathrm{C}=174.399
\end{array}\right\} \mathrm{DEA}
$$


Using the relationship

$$
\mathrm{x}=\frac{\frac{\mathrm{E} \%}{\mathrm{M}_{\mathrm{E}}}+\mathrm{Z}}{\frac{\mathrm{E} \%}{\mathrm{M}_{\mathrm{W}}}+\frac{300-\mathrm{E} \%}{\mathrm{M}_{\mathrm{W}}}}
$$

and $\oint=\frac{\mathrm{Z}}{\mathrm{E} \% / \mathrm{M}_{\mathrm{E}}}$

where $\mathrm{E} \%=\%$ ethanolamine by weight; $\mathrm{M}_{\mathrm{E}}=$ molecular wt. of ethanolamine: $\mathrm{M}_{\mathrm{W}}=$ mol. wt. of water.

determining $x$ from

$$
X P_{E}+0(I-x) P_{W} \Rightarrow \pi
$$

the hydration number was calculated. The results are tabulated in table 11. 


$$
-169-
$$




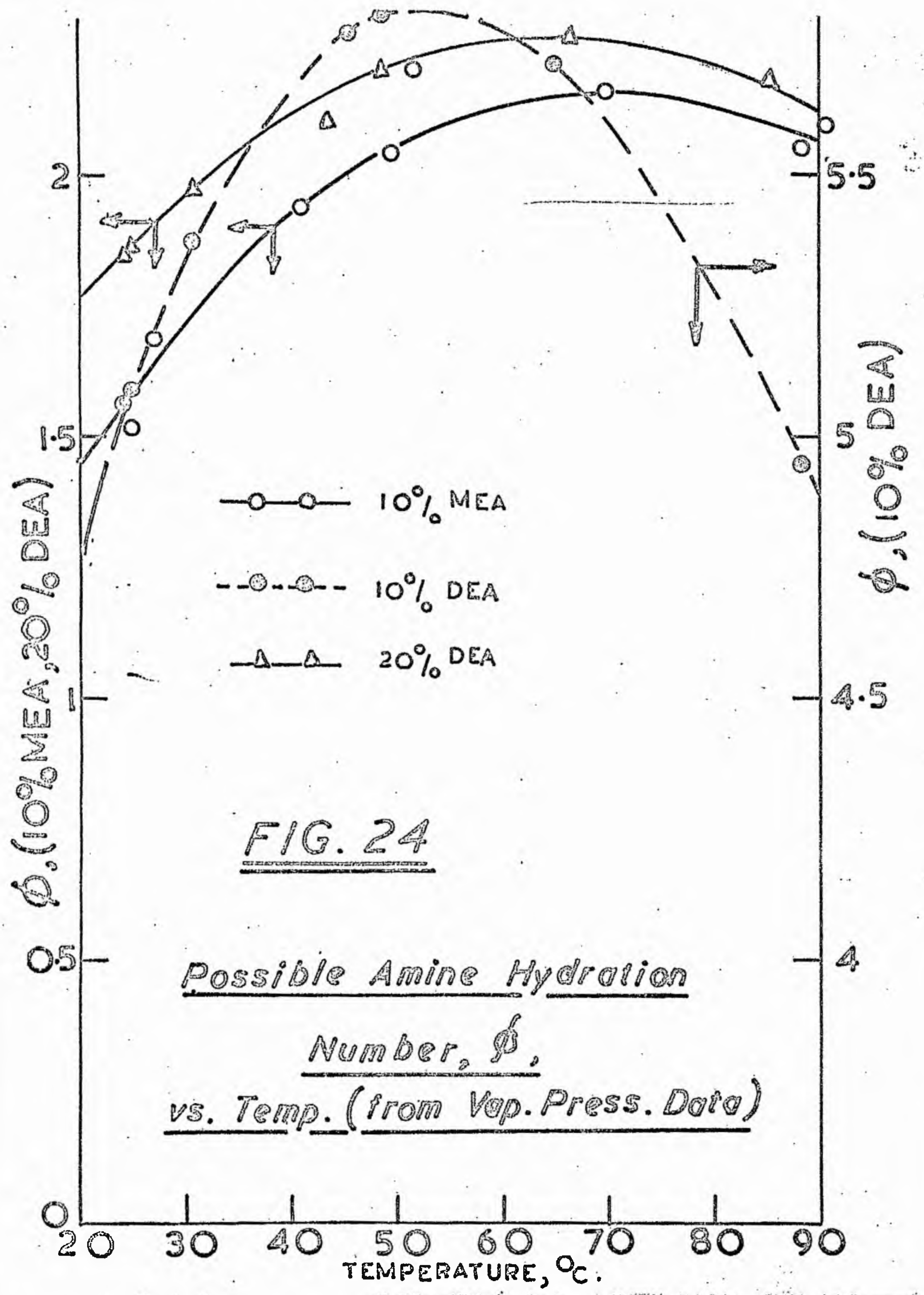


Table 11.

Possible Hydration of Fthanolamines from Vap.Press.Data.

\begin{tabular}{|c|c|c|c|c|c|c|}
\hline & $\mathrm{T}^{\circ} \mathrm{C}$ & $\pi$ & $\mathrm{P}_{W}$ & $P_{E}$ & Z & $\phi$ \\
\hline \multirow{11}{*}{$\begin{array}{l}10 \% \\
\text { MEA }\end{array}$} & 25 & 21.793 & 23.756 & 0.344 .5 & 0.2489 & 1.520 \\
\hline & 27 & 24.494 & 26.739 & 0.471 .3 & 0.2762 & 1.687 \\
\hline & $4 \pm$ & 53.025 & 58.340 & 1.303 & 0.3171 & 1.937 \\
\hline & 49.5 & 81.779 & 90.24 & 2.453 & 0,3335 & 2.037 \\
\hline & 51.6 & 90.672 & 100.6 & 2.848 & 0.3603 & 2.201 \\
\hline & 69.7 & 208.54 & 230.7 & 9.346 & 0.3529 & 2.255 \\
\hline & 69.8 & 209.44 & 231.7 & 9.380 & 0.3526 & 2.154 \\
\hline & 88.1 & 443.36 & 488.0 & 26.53 & 0.3354 & 2.048 \\
\hline & 90.5 & 486.23 & 535.82 & 30.09 & 0.3421 & 2.090 \\
\hline & 98.8 & 662.50 & 727.98 & 45.78 & 0.3315 & 2.025 \\
\hline & 99.8 & 686.97 & 754.58 & 48.08 & 0.3300 & 2.016 \\
\hline \multirow{8}{*}{$\begin{array}{l}10 \% \\
\text { DEA }\end{array}$} & \multirow[b]{2}{*}{24.3} & \multirow[b]{2}{*}{20.202} & \multirow[b]{2}{*}{22.785} & $\left(x \geq 0^{2}\right)$ & \multirow[b]{2}{*}{0.4809} & \multirow[b]{2}{*}{5.056} \\
\hline & & & & 0.0263 & & \\
\hline & \multirow{2}{*}{$\begin{array}{l}25 \\
30.9\end{array}$} & 21.047 & 23.756 & 0.0290 & 0.4842 & 5.091 \\
\hline & & 29.503 & 33.503 & 0.0627 & 0.5175 & 5.377 \\
\hline & 45.6 & 64.726 & 74.12 & 0.359 & 0.5489 & 5.771 \\
\hline & 48.6 & 75.298 & 86.28 & 0.499 & 0.5522 & 5.806 \\
\hline & 64.9 & 163.27 & 186.72 & 2.564 & 0.5431 & 5.711 \\
\hline & $84+2$ & 371.81 & 420.16 & 13.66 & 0.4898 & 5.150 \\
\hline & 25 & 20.969 & 23.756 & 0.029 & 0.3534 & 1.858 \\
\hline & 30.8 & 29.245 & 33.312 & 0.0619 & 0.3751 & 1.972 \\
\hline & 43.6 & 58.196 & 66.86 & 0.287 & 0.3999 & 2.102 \\
\hline $3.0 \%$ & 66.6 & 174.48 & 201.41 & 3.003 & $0.4+291$ & 2.256 \\
\hline TEA & 85.4 & 383.15 & 440.61 & 15.03 & 0.4139 & 2.176 \\
\hline & 99.0 & 642.33 & 733.24 & 47.94 & 0.3842 & 2.020 \\
\hline
\end{tabular}


The calculations to estimate a degree of hydration from hygroscopicity, refractive index measurements, and vapour pressure data were not entirely successful. However, using the hydration numbers calculated from hygroscopicity and the vapour pressure data, it will be off interest to compare the diffusion coefficients predicted ( from the semi-empirical equations) using a $V_{A}$ which includes a hydration number with those coefficients previously predicted. The $\phi$-values from vapour pressure data for MEA and DEA will be taken as 2 and 5 respectively. Thus the recalculated molecular volumes, $\mathrm{V}_{\mathrm{A}}^{\prime}$, will be as. in tablie 12.

\section{Table 1.20}

Estimated Hydration Numbers and Related Molecular Volumes.

\begin{tabular}{|c|c|c|c|c|c|}
\hline & \multirow[b]{2}{*}{$V_{A}$} & \multicolumn{2}{|c|}{ From Hyg.data } & \multicolumn{2}{|c|}{ From v.p.data } \\
\hline & & $\phi$ & $\mathrm{V}_{\mathrm{A}}^{\prime}$ & $\phi$ & $V_{A}^{\prime}$ \\
\hline MEA & 73.4 & 3.2 & 733 & 2 & 111 \\
\hline $\mathrm{DEA}$ & 7.26 .7 & 4 & 202 & 5 & 221. \\
\hline TEA & 180.0 & 4.2 & 260 & & \\
\hline
\end{tabular}

Uising these $V_{A}^{\prime}$ values, the diffusion coefficients predicted by the Wilke-Chang, Scheibel, and othmer-Thakar equations will be as in table 13 . 
Table 1:3。

Predicted Diffusivities Using Estimated Hydration Numbers.

\begin{tabular}{|c|c|c|c|c|c|c|c|}
\hline & & \multicolumn{2}{|c|}{ No.Hydr. } & \multicolumn{2}{|c|}{ from hygr. } & \multicolumn{2}{|c|}{ Prom $v_{0} p_{0}$} \\
\hline & & $D \times 10^{6}$ & \%error & $D \times 10^{6}$ & \%error & D $\times 10^{6}$ & \%error \\
\hline \multirow{3}{*}{ MEA } & Wilke & 12.89 & +28 & 9.13 & -12 & 10.0 & -4 \\
\hline & Sch. & 12.55 & +21 & 8.4 .1 & -19 & 9.35 & $=10$ \\
\hline & oth. & 12.10 & +16 & 8.55 & -18 & 9.42 & -10 \\
\hline \multirow{3}{*}{$D E A$} & Wilke & 9.285 & +40 & 7.01 & +6 & 6.64 & +0.25 \\
\hline & Sch. & 8.668 & $+3 I$ & 6.67 & +0.7 & 6.36 & -4 \\
\hline & Oth. & 8.714 & +32 & 6.60 & 0 & 6.24 & -5.5 \\
\hline \multirow{3}{*}{ TEA } & Willke & 7.521 & +17 & 6.13 & -4.5 & & \\
\hline & Sch. & 7.115 & +11 & 5.84 & -9 & & \\
\hline & Oth. & 7.058 & +9.9 & 5.74 & -10.5 & & \\
\hline
\end{tabular}

(all Drvalues, above in $\mathrm{cm}^{2} / \mathrm{sec}$ ) 
From table 13, it can be seen that the inclusion of a hydration number in the calculation of the ethanolamine molecular volume brings the diffusivities predicted by the semi-ernirical equations into much better agreement with the experimental diffusivities. In view of this improved correlation, it would appear that where abnormalities are known to occur in solution, an allovance should be made for this when calculating the molecular volumes for use in the semi-empixical equations. This is somewhat of a s.topngap measure, for the semi-empirical equations would appear to be unreliable in any case.

It is hoped that in the future, with an increasing volume of accurate diffusion measurements to hand, an accurate semi-empirical equation can be formulated which allows for molecular irregularities in solution. In the meantime, it is suggested that for any known molecular irregularity, allowance for this should be made in calculating the molecular volumes for use in the semi-empirical equations. 


\section{$-174 m$}

\section{SUMMARII}

The wavefront shearing optical interferometer, as constructed in this laboratory; has proved to be a highly accurate and sensitive instrument. A new method of computation has been devised, based on the method of least squares, and necessitating the use of a computer, which has facilitated the calculation of diffusivity (with increased accuracy ).

The programme of investigation of diffusivity in Iiquid-Iiquid systems, using the wavefront shearing: interferometer, involved the systems ethanolamines-water (MEA, DPA, and TEA - water ). The accuracy of the interferometer was established by determining the diffusion coefficient for djlute sucrose-water solutions, this giving a satisfactory comparison with established results. Diffusion coefficients for the ethanolamine-water systems were measured over a range of concentrations.

The diffusivities of the ethanolamines, as measured in this work, were then compared with the values obtained by Thomas and Furzer ( 1 ) ( 2 ) using a Gouy interferometer. Their calculations were chocked, and it was established that their results were only of limited accuracy. 
However, the diffusivity values for dilute ethanolamine solutions as measured by the above woxkers wone alnost 1 Identical with those obtained in this study. Also, the diffusivitymconcentration curves for DEA and TEA are similar in the two cases.

It is important to note that the diffusivities of the dilute ethanolamine solutions are in agreement, for it was these values that excited critical comment from Ibrahim and KuIoor ( 3 ). In view of this criticism, the application of diffusional theory (especially the existing: semi-empirical equations ) to the ethanolamine-water systems was thoroughly examined. From this appraisal, two definite. conclusions may be drawn; -

a) the semi-empirical equations are in themselves highly unsatisfactory. The average accuracy of the equations is in no case better than $10 \%$, and reported deviations aro as high as $30 \%$ in error in predicting diffusivity. The failings of these equations were pointed out, one of the chief weaknesses being the inclusion of many eluctrolytic and non-ideal systems for formulating the equations, yet without making allowances for molecular irregularities in solution.

b) the ethanolamines are hydrated in solution to 
an appreciable extent. Diethanolanine, in particualr, exhibits anomalous behaviour (as shown by viscosity measurements). In an attempt to ascertain the degree or hydration, calculations were made using hygroscopicity, refractive index, and vapoux pressure data for the ethanolanines in solution. No definite hydration number was determined, but the hydration numbers calculated were usec in recalculating the molecular volumes for use in the semi-empirical equations.

It was shown that by accounting for molecular irregularities ( by increasing the molecular volume) when applying the semimempirical equations, a much more satisfactory agreement is obtained between predicted and observed. diffusivity values。

A possible indication of the necessary increase in the molecular volume is shown by the constant $A_{1}$ in Arnold's equation. This fact may be coincidental, but deserves a slightly Iess cursory investigation.

It is suggested that, in future, when the semim -empirical equations are used to predict diffusivitys that some consideration be made as to any molecular ixregularIties in solution. A far more satisfactory outcome would be a complete re-examination of the theories of diffusion in liquids, with the supplementing of these semi- 
mempirical equations by equations based on ideal systems which take into account any molecular irregularities.

VII(a) Suggestions for Further Work.

The optical arrangement of the wavefront shearing. intenferometer is perfectly satisfactory, and needs no alteration. Likewise, the recording and measurement of the fringe pairs, and the calculation of the diffusivity need no improvenent.

The only experimental improvement that could be made, though it is not essential, is the redesign of the flowing-junction cell. The following improvements could be made with the celli;-

i) Inclusion of a baffle chamber between the mencury reservoir and the liquid reservoirs. This would eliminate any vibration transmitted by the mercury when turning off the mercury tap at the start of diffusion.

ii) Retention of the present double chamber axrangement, but the inclusion of two adjustable exit slits in each chamber. The adjustable slits would be engineered from knifemedges vertically movable by micrometer 
screwmothreads. This arrangement would enable an extremely sharp interface to be obtained.

iii) Fabrication of all the glasswork in a pressure tight "Quick-fit" type arrangement. At the moment, cleaning. the glasswork is laborious and not very efficient since all the glasswork is in one unit. However, if the reservoirs and taps could individually be quickly removed, the setting up of the cell for experimentation would be greatiy facilitated.

The possibilities of the application of the waverront shearing interferometer to liquid-liquid systems is endless. Even today, diffusional investigations are being made ( 73 ) using diaphragm cells, which are considerably less convenient and accurate to use than this interferometer. With the superiority of the interferometer, a wealth of userul data could be rapidly accomplished.

The wavefront shearing interferometer also has great potential use for thermal diffusion measurements. BryngdahI ( 74 ) has pioneered the interferometer's use for this purpose, and Guistafsson et. al ( 75 ) have recently started work in this field.

One main project which exploits the novel use of the wavefront shearing interferometer is already under way. 
This is the application of the wavefront shearing interferometer to gasmliquid diffusion. Because of the supexior sensitivity of the instrument, this is the rirst time that an interferometer has been used in this rield with any measure of success.o This application, which has immensely exciting possibilities, is described in Part II of this work.

Other recent developements include the micro-wedge interferometer ( 76 ) (77) and Moiré intexferometry ( 78 ) ( 79 ), both of which it is hoped to investigate in this laboratory at a later dato.

There is one other field of investigation that is not being studied yet, but which must be thoroughly examined in the future. This is the subject of Iiquid diffusion theory, - and in particular the formulation of a new semi-empirical equation which will erable diffusivity to be predicted a little more successfully than at present. It is suggested that the basis of this. work take the form of,

a) amassing diffusivity data for systems as near to ldeal as possible,

b) formulating an equation from these carefully vetted systems, and then

c) introducing a molecular irregularity factor into 


\section{$=0.180 \mathrm{mon}$}

this equation that would account for deviations in abnormal Iiquid systems.

As mentioned above, the wavefront shearing Interferometer is being applied in this laboratory to gas-liquid diffusion. This quite novel developement will now be described in Part II of this work. 


$$
-1.8 I-
$$

PART II.

THW APPLTCATION OF THE WAVEFRONT SHEARING INTERFEROMETER

to.

GAS-LIQUID DIFFUSTON MEASUREMENTS. 
TNTRODUUCI ION。

With the wavefront shearing interferometer built, and proved as being a highly accurate and sensitive instrument for liquid-liquid diffusion measurements, it is a natural progression to apply the interferometer to gas-1iquid diffusion.

Finstly, for the physical absorption of a gas into a Liquid, the possibility of measuring $D_{A}$ (the diffusion coefficient of the gas into the liquid) by an interferometric technique is an attractive proposition. The most convenient existing methods for the determination of diffusion coefficients of gases in Iiquids involve the measurement of mass transfer rates under unsteadymstate conditions, and suffer from the inherent alsadvantage of requiring flowing: systems. The Iaminar jet technique which has been used in this laboratory by Thomas and Adams ( 80$)(81$ ) is an accurate means of determining mass transfer rates, but a degree of error is introduced by the presence of entry and end effeots.

Thus, if it were possible to absorb a gas into a liquid ( with no reaction) and to calculate $D_{A}$ from interferometric measurements of the concentration profiles 
made"In situ", and without necessitating an extensive knowledge of the physical properties of the system, then a useful technique would be evolved.

Secondly, for absorption of a gas into a liquid accompanied by a chemical reaction, it would again be highly desirable to be able to make measurements "in situ". In absorption accompanied by a rapid second-order irreversible reaction, all existing theories account for the formation and movement of a"reaction zone". The movement of this reaction zone is related to the physical properties of the system, and by calculating this movement, the mass transfer rate may be predicted. Previous investigations, using such apparatus as the laminar jetghave produced good agreement between experimental results and theory. Using the wavefront shearing interferometer, it may be possible to observe the aatual movement of this reaction zone in a stagnant system, since a modification to the interferometer enables a direct observation of concentration profiles to be made. Thus, the presence of the reaction zone can be confirmed by direct observation, and a possible means is formulated of relating. theory to practice.

There are limits to the applicability of interferometry to gas-liquid absorption. The first consideration 
is the sensitivity of the interferometer in relation to the system under investigation. This will be discussed in some detail further on. Another consideration is the presence of heat effects associated with the absorption of a gas into a liquid. The elimination of these effects will be to a large degxee experimental, and this again will be discussed in the relevant section.

A survey of the theories relevant to physical absorption and absorption accompanied by a rapid second-order irreversible reaction follow.

In the experimental investigations so far undertaken In this laboratory, the following two systems have so far been investigated:-

1) Carbon Dioxide into water(physical absorption).

2) Carbon Dioxide into dilutie monoethanolanine solution ( absorption with fast 2nd order reaction) The results of these investigations are reported. 
I. THEORY OF GAS-LIQUID DTFEUSION.

I(a) Gas Absorption Without Reaction.

The theories for the absorption of a gas by a Iiquid without chemical reaction may be broadly classified under I) Film theory

2) Penetration Theory.

Film Theory

Whitman ( 82 ) proposed the two film concept, whereby at the interface between a gas and a liquid it could be assumed there were two films adjacent to the interface. (see Fig.Ii)

The rate of mass transfer is defined by the following equations

$$
N_{A}=K_{I}^{O}\left(c_{A i}-c\right)=K_{G}\left(p-p_{i}\right) \ldots \ldots(I)
$$

and the Iiquid film mass transfer coefficient is defined by

$$
\mathrm{K}_{I_{1}}^{O}=\frac{\mathrm{D}_{A}}{\mathrm{x}_{I_{1}}}
$$




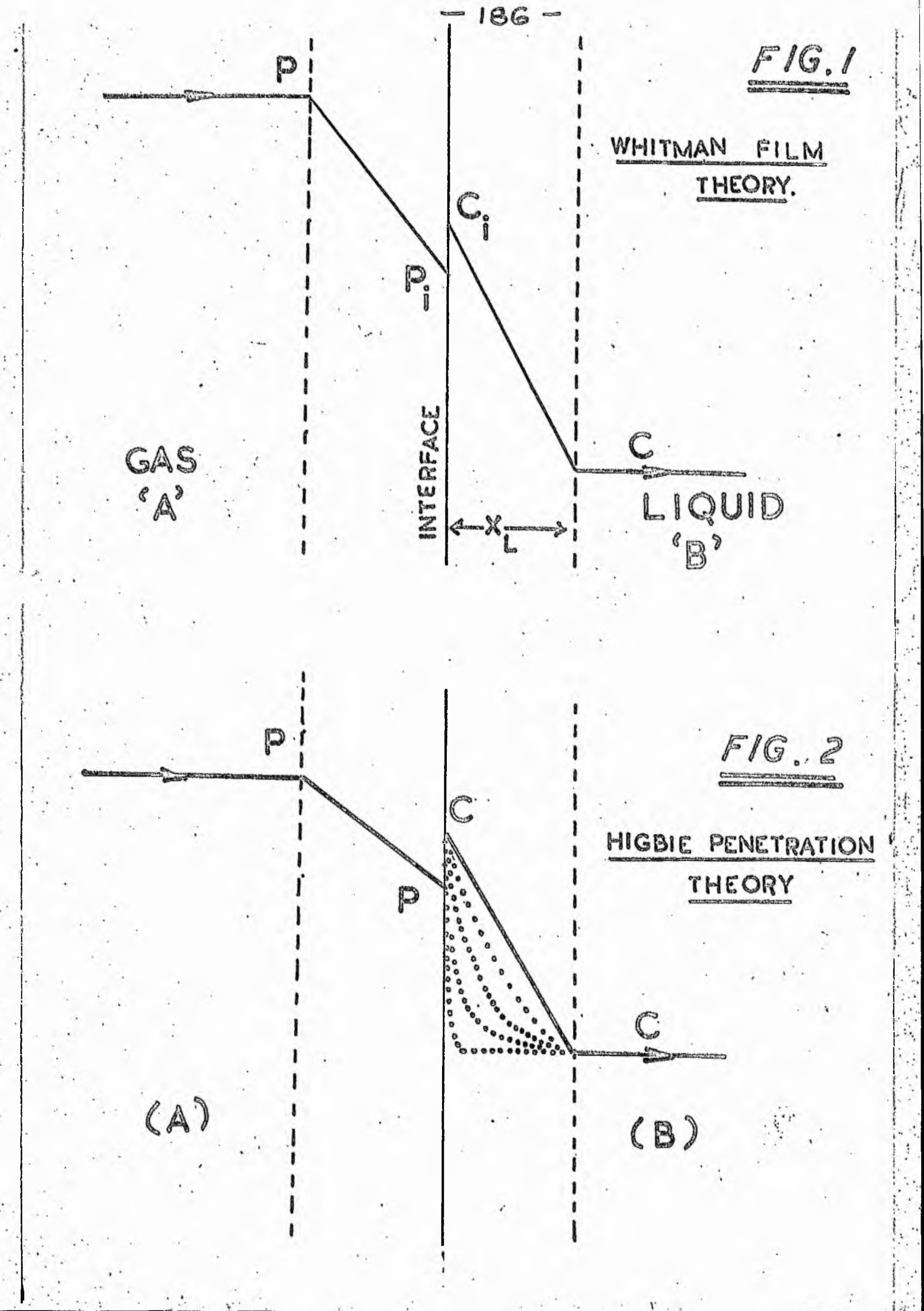


Penetration Theory.

Higbie ( 83 ) showed that the Whitman film mechanism was onliy true if the penetration of the liquid film by the gas continued uninterrupted until steady flow could be assumed ( see Fig. 2 ).

The dotted lines in Fig.2 represent the concentration profile of $A$ and $B$ in the initial penetration process. From a solution of Fick's second law for the appropxiate boundary conditions, Higbie obtained

$$
\frac{c}{{ }^{c} A i}=\frac{1}{\sqrt{\pi D t}} \int_{x}^{\infty} e^{-x^{2} / 4 D t} d x
$$

which is $\frac{c}{c_{A i}}=\operatorname{erfc}(x / 2 D t)$

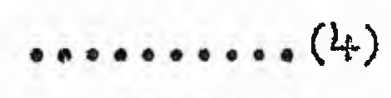

A similar solution is given by Crank ( 1.0 ).

From this, $\mathrm{K}_{\mathrm{L}_{\mathrm{L}}}^{\mathrm{O}}$ is defined by

$$
\mathrm{K}_{\mathrm{L}}^{\mathrm{O}}=2 \sqrt{\frac{\mathrm{D}_{\mathrm{A}}}{\pi^{t}}}
$$


$I(b)$. Gas Absorption With Reaction.

The monograph by Sherwood and Pigford ( 84 ) gives an account of the theories applicable to absorption with reaction. There are two theoretical bases for the theoretical discussion of the kinetic theory of simultaneous diffusion and chemical reaction;

a) assuming a physical film of negligible capacity but finite resistance (film theory).

b) assuming unsteady state molecular diffusion of the solute ( penetration theory).

Rapid second order reactions only will be considered in this section.

F'ilm Theory, -2nd Order Reaction (Hatta).

Assume $\begin{gathered}\mathrm{A} \\ \text { (gas) }\end{gathered}$

A dissolves and reacts with $B$ at the phase boundary: the product $A B$ begins to diffuse towards the main boundary of the Iiquid; as $B$ is depleted near the interface, A diffuses further into the bulk of the Iquid to meet $B$ diffusing in the opposite direction. Thus the reaction zone moves away from the interface, until after a short while the zone reaches its equilibriun position (see Fig.3). 


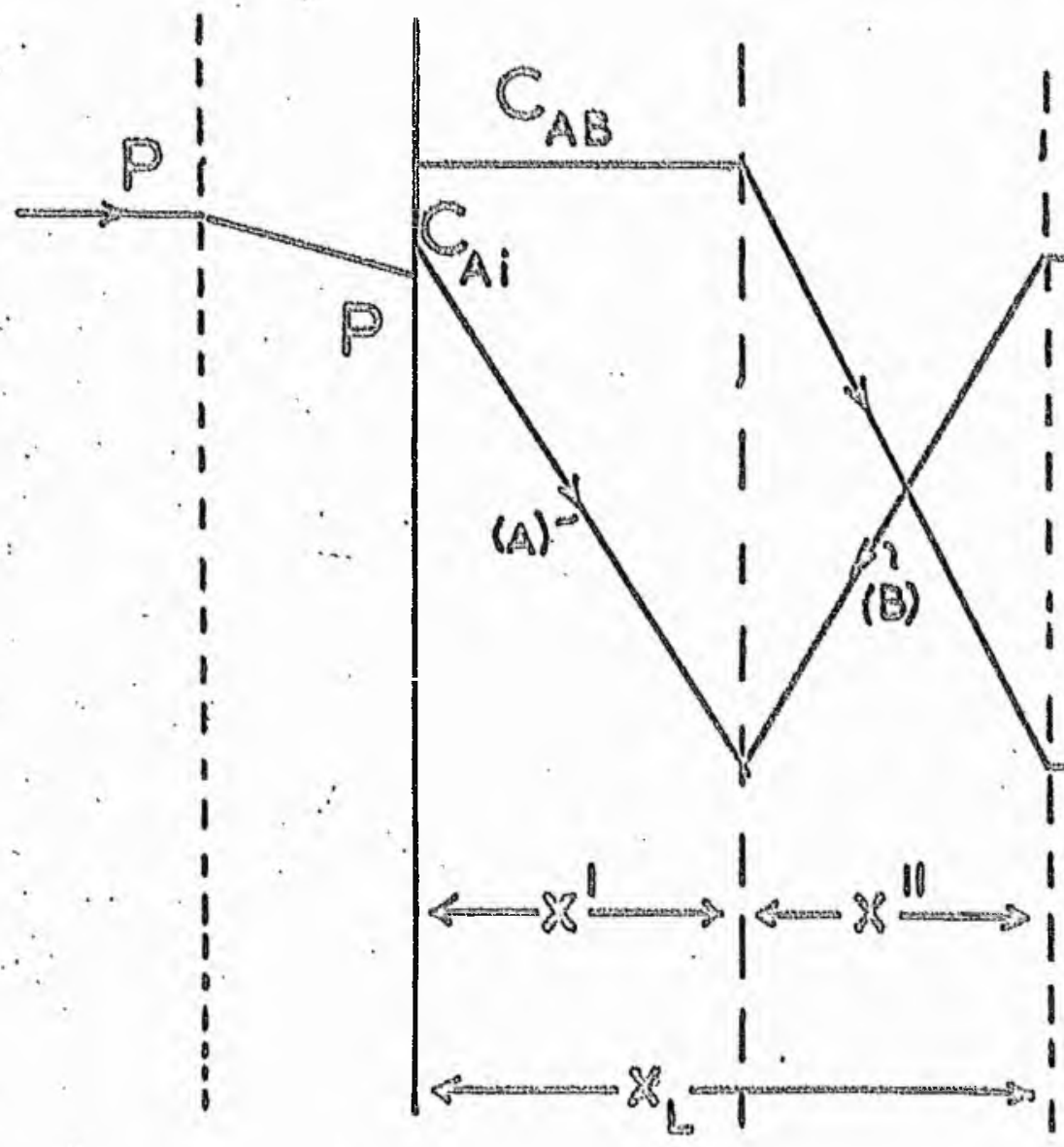

819.3

H0880 Pilno Theory.

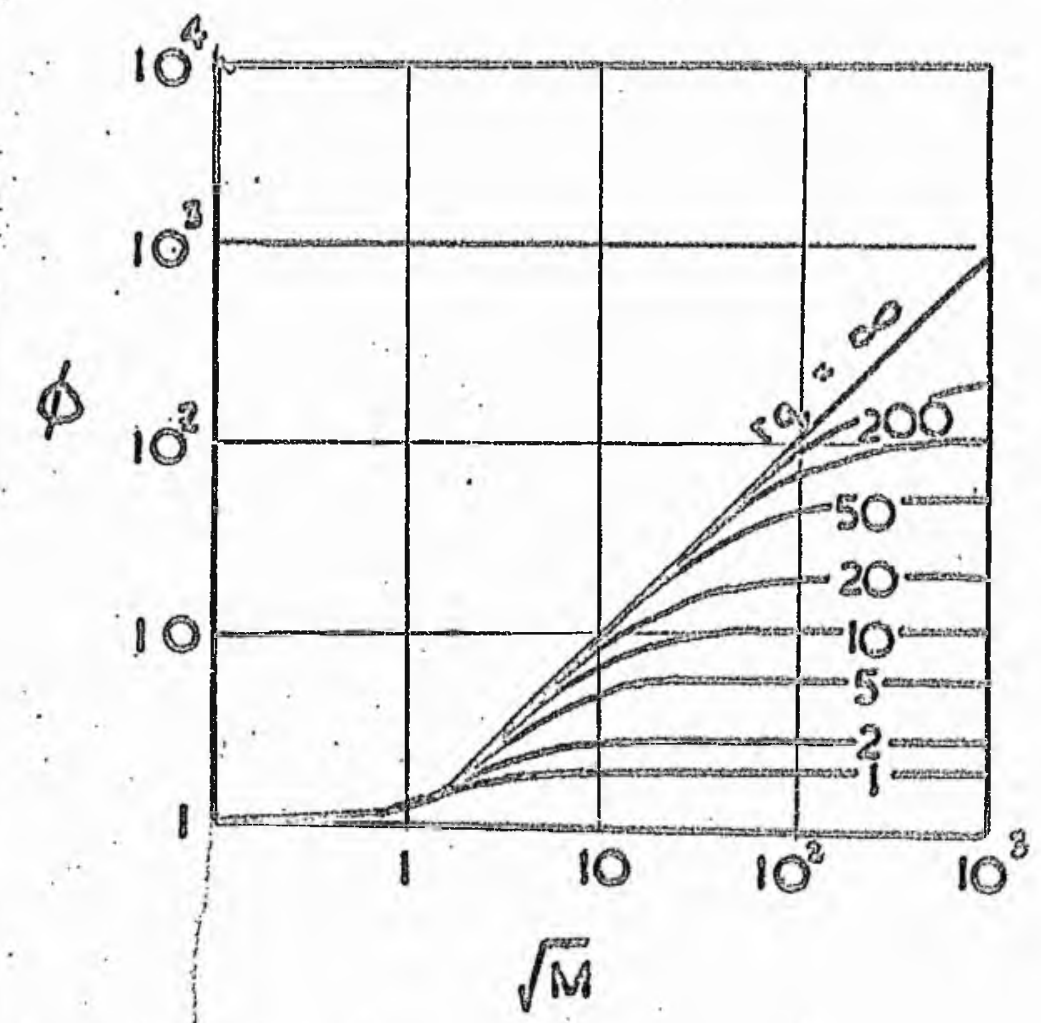

$519 \cdot 8$

Curves or

of US 
For the gas film,

also $\quad \mathbb{N}_{A}=x_{L}^{0}\left(c_{A i}-0\right)=\frac{D_{A}}{x^{3}}\left(c_{A i}-0\right)$

$$
\mathbb{N}_{A}=K_{G}\left(p-p_{i}\right)
$$

and $\quad-\mathbb{N}_{B}=\frac{D_{B}}{x^{\prime \prime}}\left(c_{B o}-0\right)$

By assuming Henry's Law, $\mathrm{p}_{1}=\mathrm{HHC}_{\mathrm{A} 1}$, combining equations $6,7,888$, and eliminating gas phase concentrations,

$$
N_{A}=\frac{c_{A i}+\left(D_{B} / D_{A}\right) c_{B O}}{x_{L / D_{A}}}
$$

and hence, $\mathrm{K}_{\mathrm{L}}=\frac{\mathrm{D}_{\mathrm{A}}}{\mathrm{x}_{\mathrm{L}}}\left[1+\left({ }_{\mathrm{B} / \mathrm{D}_{\mathrm{A}}}\right)\left(\frac{\mathrm{c}_{\mathrm{BO}}}{\mathrm{C}_{\mathrm{AI}}}\right)\right]$

$$
\text { or, } \begin{aligned}
\phi=\frac{K_{L}}{K_{L}} & =I+\left({ }^{D_{B}} D_{D_{A}}\right)\left(\frac{c_{B O}}{{ }_{A} i}\right) \\
& =I+r \cdot q
\end{aligned}
$$

where $\phi$ is the ratio between the mass transfer coefficients. with and without reaction, and

$$
r=\left(\frac{D_{B}}{D_{A}}\right), q=\left(\frac{C_{B O}}{C_{A I}}\right)
$$

By dimensional analysis, Van Krevelen and Foftyzex (85) (86) related to the dimensionless products 


$$
\sqrt{M}={\frac{\left(K D_{A} c_{B O}\right.}{K_{L}^{0}}}^{-191 \ldots} \text { and } q
$$

where $\mathrm{k}$ is the reaction velocity constant in

$$
-\frac{d A}{d t}=-\frac{d B}{d t}=k_{0} A B
$$

Brian (87) has shown that the film theory predicts that.

$\phi$ is a unique function of $\sqrt{M}$, and $r \cdot q$, and shows that the film theory solution may be approximated by

$$
\phi=\frac{\sqrt{\mathbb{M}} \sqrt{1-\frac{\phi-1}{r q}}}{\tanh \sqrt{M} \sqrt{\left(1-\frac{\phi-1}{r q}\right)}}
$$

$\tanh x=\frac{e^{x}-e^{-x}}{e^{x}+e^{-x}}$, and for $x>2, \tanh x=1$

This relationship between $\phi, \sqrt{\mathrm{M}}$, and $x q$ is described by Gilliland et al ( 88 ), Brian eti al ( 89 ), and Thomas.(8I), who have plotted if against $\sqrt{M}$ with $I+r * q$ as parameter ( see Fig. 4).

From the curves relating $\phi$ to $\sqrt{M}$ the following points may be observed;

a) at. low values of $\sqrt{M}(\operatorname{small} k), \phi$ tends to unity. Thus a slow reaction will not increase the mass transfer coefficient. 
b) At large values of $\sqrt{M}(x$ is large $), 6$ approaches an asymptote for each $x \cdot q$ value. This asymptote corresponds to an infinitely repid reaction region, and is given by

$$
\phi_{a}=1+r \cdot q
$$

c) For any fixed value of $\sqrt{\mathrm{M}}, \phi$ approaches an asynptote for large values of $r . q$. As this asymptote is approached, $c_{B O}$ is essentially constant throughout the Iiquid phase and the reaction becomes pseudo first order. The curve of the asymptote is closely approximated to by the equation

$$
\begin{aligned}
\phi & =\sqrt{M} \\
\text { whence } \quad \mathrm{K}_{\mathrm{I}} & =\sqrt{\mathrm{K} \cdot \mathrm{D}_{\mathrm{A}}{ }^{\mathrm{c}}{ }_{\mathrm{Bo}}}
\end{aligned}
$$

Penetration Theory, - Rapid 2nd order Irreversible Reaction.

The derivation for the theory for unsteady state absorption with rapid second order reaction is described adequately by Sherwood and Pigford ( 84 ).

The model for the diffusion of $A$ into $B$, with 
reaction $A+B \rightarrow A B ;$, is similar to the film theory model of Hatta. As $A$ is absorbed, B molecules near the interface are consumed in the reaction and are replenished by diffusion of additional $B$ molecules from the main body of the Iiquid. Since the reaction velocity is assumed infinite, the assumption follows that reaction is instantaneous when $A$ and $B$ are brought together, and thus the point where $A$ and $B$ meet. each other will move furthex away from the interface. The rate of absorption of $A$ therefore: grows progressively smaller as the molecules have to diffuse funther into the liquid in order to react. Since A does not react with $B$ at a distance $05 x<x^{\prime}$ ( where $x^{\prime}=$ distance of the reaction zone from the interface), and $B$ does not react at $x^{1}<x<\infty$, then the diffusion of $A$ and $B$ are governed by

$$
\begin{aligned}
D_{A} \cdot \frac{\partial^{2} c_{A}}{\partial x^{2}} & =\frac{\partial c_{A}}{\partial \theta} \\
D_{B} \cdot \frac{\partial^{2} c_{B}}{\partial x^{2}} & =\frac{\partial c_{B}}{\partial \theta}
\end{aligned}
$$

where $\theta=$ time.

In Fig.5, concentration profiles for $A$ and $B$ are shown at times $\theta$ and $\theta+d \Theta$, the reaction zone having moved $d x^{2}$ in time $\left.d\right\}$. 


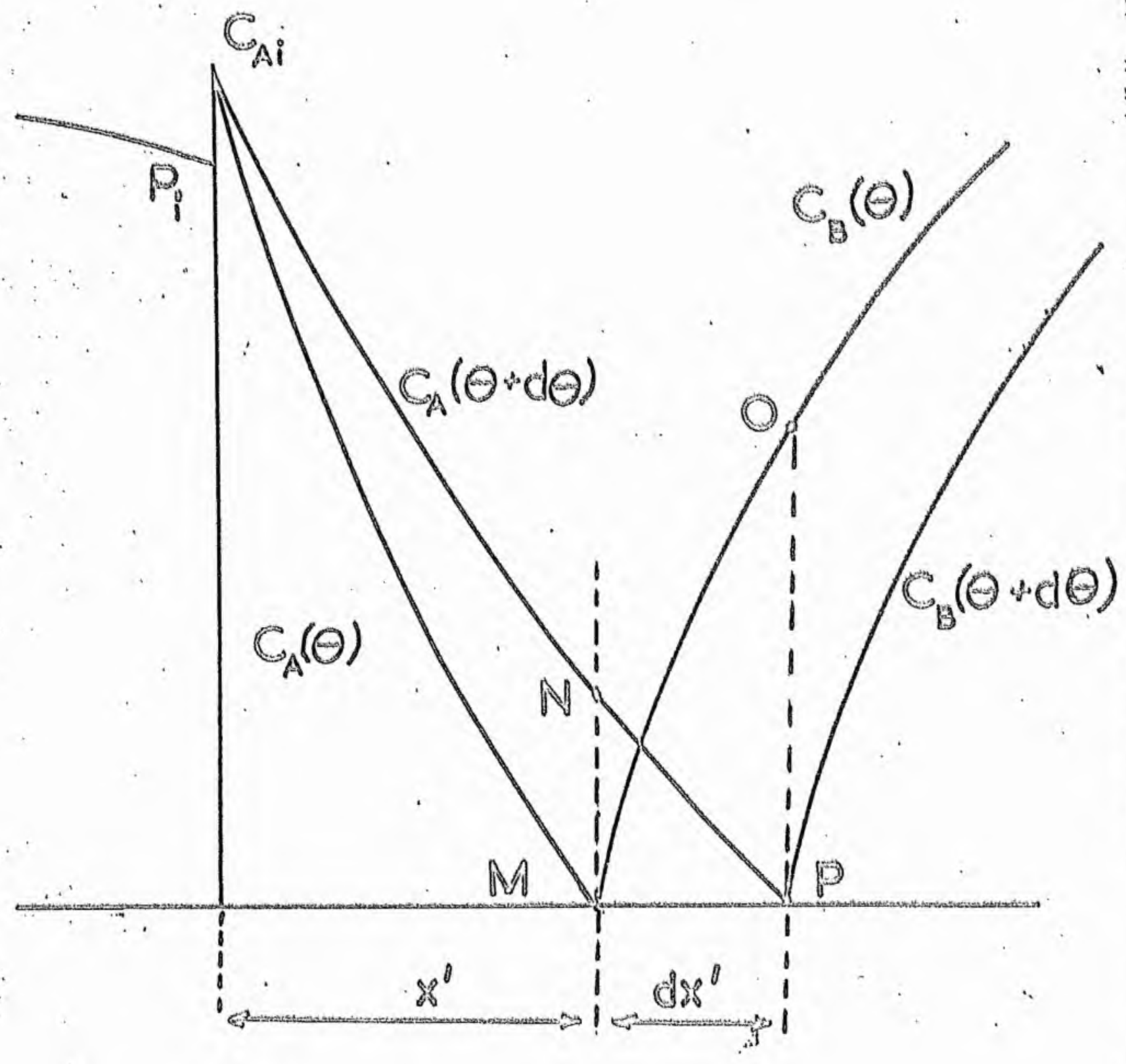

Fla. 5

Penerrarion Treory Model pos

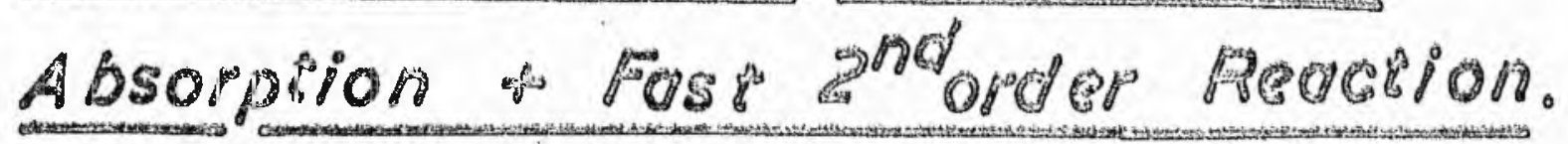


From the geometry of the triangles MNP and MPO (Fig.5),

$$
\begin{aligned}
& \text { MN }=\frac{\partial c_{A}}{\partial \theta} \cdot d \theta=-\frac{\partial c_{A}}{\partial x} \cdot d x^{\prime} \\
& O P=-\frac{\partial c_{B}}{\partial \theta} \cdot d \theta=\frac{\partial c_{B}}{\partial \theta} \cdot d x^{\prime}
\end{aligned}
$$

From 20 and 21 ,

$$
d x^{2} / d \theta=-\frac{\partial c_{A}}{\partial \theta} / \frac{\partial c_{A}}{\partial x}=-\frac{\partial c_{B}}{\partial \theta} / \frac{\partial c_{B}}{\partial x}
$$

Solutions of equations 18 and 19 to satisfy the necessary boundary conditions are ;-

$$
\begin{aligned}
c_{A} & =A_{I}+B_{1} \operatorname{erf} \frac{x}{\sqrt[2]{D_{A} \theta}} \\
c_{B} & =A_{2}+B_{2} \operatorname{erf} \frac{x}{\sqrt[2]{D_{B} \theta}} \\
\text { where exf } z & =\sqrt[2]{\pi} \int_{0}^{z} e^{-t^{2}} \cdot d t
\end{aligned}
$$

Differentiating 23 and 24 with respect to $x$ and $\theta$, substituting intio 22 , and putting $x=x^{1}$, we get 


$$
\frac{d x^{2}}{d \theta}=\frac{x^{2}}{2 \theta}
$$

On integrating

$$
x^{2}=2 \sqrt{\alpha} \sqrt{\theta}
$$

where $2 \sqrt{x}$ is the integration constant.

Equation 27: therefore relates the movement of the reaction zone to time.

The constant $\sqrt{\alpha}$ is determined by considexing the boundary conditions .

$$
\begin{aligned}
& \text { i.e. I./ } c_{A}=c_{A i}, x=0, \theta>0 \text {. } \\
& \text { 2.1 } c_{B}=c_{B O}, x=\infty, \theta \geqslant 0 \text {. } \\
& \text { 3.1 } c_{\mathrm{B}}=c_{\mathrm{BO}}, \theta=0, x>0 \text {. } \\
& \text { 4./ } c_{A}=0, c_{B O}=0 \text {, when } x=x^{8}, \theta>0 \text {. } \\
& 5 . / D_{A} \frac{\partial c_{A}}{\partial x}+D_{B} \frac{\partial c_{B}}{\partial x}=0 \text {, when } x=x^{\prime}
\end{aligned}
$$

Applying the boundary conditions to equations 22,23, 24, 26 , and 27 to solve for the constants $A_{1}, A_{2}, B_{1}, B_{2}$ and $\alpha$,

$$
\begin{aligned}
A_{1} & =c_{A I} \\
A_{2}+B_{2} & =c_{B O}
\end{aligned}
$$




$$
\begin{gathered}
A_{1}+B_{1} \operatorname{erf}\left(\frac{\alpha}{D_{A}}\right)^{\frac{1}{2}}=0 \ldots \ldots(30) \\
A_{2}+B_{2} \operatorname{erf}\left(\frac{\alpha}{D_{B}}\right)^{\frac{1}{2}}=0 \ldots \ldots\left(3 I_{1}\right) \\
B_{I} \sqrt{D_{A}} e^{-\left(\alpha / D_{A}\right)}+B_{2} \sqrt{D_{B}} e^{-\left(\alpha / D_{B}\right)}=0 \ldots(32)
\end{gathered}
$$

Solving eqns. 28 to 32

$$
\begin{gathered}
\left(D_{B /}\right)_{A}^{\frac{1}{2}}\left(\frac{\left.C_{B O}\right)}{c_{A j}} e^{\left(\alpha / D_{A}\right)\left(I-D_{A} / D_{B}\right)} \operatorname{erf}\left(\frac{\alpha}{D_{A}}\right)^{\frac{1}{2}}+\operatorname{erf}\left(\frac{\alpha}{D_{B}}\right)^{\frac{1}{2}}=1\right. \\
\ldots \ldots(33)
\end{gathered}
$$

ox, $\sqrt{x \cdot q} e^{\sigma_{A}^{2}(I-I / x)} \operatorname{erf} \sigma_{A}+\operatorname{exf} \sigma_{A}(1 / \sqrt{x})=I \ldots(33 a)$

$$
\text { where } \sigma_{A}=\frac{\sqrt{\alpha}}{\sqrt{D_{A}}} \text { and } x=\frac{D_{B}}{D_{A}}
$$

Thus the integration constant $\sqrt{\alpha}$, from eqn. 27 , describing the movement of the reaction zone with time, is given by the implicit function described by equation 33

Dankwerts ( 90 ) has presented a similar equation to 33, but without any discussion or mathematical developement. The equation Dankwerts gives is, 


$$
\begin{array}{r}
\left(\frac{c_{A i}}{\sqrt{D_{B}}}\right) \sigma_{B}^{2} \operatorname{exfc} \sigma_{B}-\left(\frac{c_{B o}}{\sqrt{D_{A}}}\right) \theta_{A}^{\sigma_{A}^{2}} \operatorname{ert} \sigma_{A}^{2} e^{\sigma_{A}^{2}}=0 \\
\ldots .(34)
\end{array}
$$

Rearranging this equation by putting

$$
\sigma_{B i}=\sigma_{A / \sqrt{r}}
$$

$$
\text { and } \operatorname{erfc} z=1-\operatorname{erf} z
$$

the same result is obtained as with equation 33

The rate of mass transfer, $\mathbb{N}_{A}$, is given by

$$
\mathbb{N}_{A}=-D_{A}\left(\frac{d c_{A}}{d x}\right)_{x=0}
$$

From oquations 23,28 , and 30 , this is equal to

$$
\mathrm{N}_{\mathrm{A}}=\frac{\mathrm{c}_{\mathrm{Ai}}}{\operatorname{art}\left(\frac{\alpha}{\mathrm{D}_{A}}\right)^{\frac{1}{2}}} \cdot\left(\frac{\mathrm{D}_{\mathrm{A}}}{\operatorname{\pi r} \theta}\right)^{\frac{1}{2}}
$$

and the average rate of absorption up to time $\theta$ is given by'

$$
\left(N_{A}\right)_{\text {av }}=\frac{c_{A i}}{\operatorname{exf}\left(\frac{G}{D_{A}}\right)^{\frac{1}{2}}} \cdot 2\left(\frac{D_{A}}{\pi \theta}\right)^{\frac{2}{2}} \ldots \ldots(37)
$$

If the liquid film mass transfer coefficient, $\mathrm{K}_{\mathrm{I}}$, is defined on the basis of a driving force $\left(c_{A 1}-0\right)$, we get 


$$
\mathrm{K}_{\mathrm{L}}=\frac{2}{\sqrt{\pi}} \cdot \frac{\left(\mathrm{D}_{\mathrm{A} / \theta}\right)^{\frac{1}{2}}}{\operatorname{exf} \sigma_{\mathrm{A}}}
$$

This same result is given by Dankwerts also, but without any developement.

Bxian (87) has predicted from the penetration theory that $\phi$ is a unique function of the variabies $\sqrt{M}$, $x$, and $q$. From the preceding section, it will be remembered that for the film theory $\phi$ is a function of $\sqrt{M}$, and $r . q$. For the penetration theory there is no mathematical solution relating $\phi, \sqrt{M}, r$, and $q$ when $r \neq 1, k \neq \infty$. As an approximation,

$$
\text { putting } \phi_{a}-1=r \cdot q
$$

$$
\text { where } \phi_{\mathrm{a}}=I / \operatorname{erf} \sigma_{\mathrm{A}}
$$

and $q \sqrt{x}=I-\left(\frac{\sigma_{A}}{\sqrt{x}}\right)$

$$
\operatorname{erf} \sigma_{A} e^{\sigma_{A}^{2}}(1-1 / x)
$$

then

$$
\phi=\frac{\sqrt{M} \sqrt{1-\frac{\phi-I}{\phi a^{-I}}}}{\tanh \sqrt{M} \sqrt{1 \cdot-\frac{\phi-I}{\phi a^{-I}}}}
$$

The curves relating $\phi$ to $\sqrt{M}$, with $\phi_{a}-1$ as parameter, axe as in Fig.4, and the same observations to these curves apply as with the film theory. 
II. THE EXPERIMENTAL INVESTIGATION OF

GAS-LIQUID DIFFUSTON.

II ( a). Sensitivity of the Wavefront Shearing Interferometer.

As mentioned in the introduction, the sensitivity of the wavefront shearing interferometer will be a prime consideration if the interferometer is to be successful for a particular system Genexaly speaking, the refractive index of a Iiquid saturated with a moderately soluble gas is negligibly different from that of the pure liquid. The penetration theory for physical absorption, eqn 4 , gives that

$$
\frac{c}{c_{A i}}=\operatorname{errc}\left(\frac{x}{2 \sqrt{D t}}\right)
$$

Assuming, again, that the refractive index/concentration relationship is a straight line, then,

$$
\frac{n}{n_{A i}}=\operatorname{exfc}\left(\frac{x}{2 \sqrt{D t}}\right)
$$

Theoretically, with the wavefront shearing interferometer using. a $6.3 \mathrm{~cm}$ cell and the mercury green Iine of $5461 \AA$, the minimum refractive index difference 
measurable, $\Delta n_{\min }=9 \times 10^{-6}$

Thus, for any gasmliquid system the refractive index difference introduced by contact between the gas and the liquid must be above this figure. For physical absoxption, $n_{\text {solvent }}-n_{A 1}$ must be above this minimum. In absorption with reaction, due to the prescence of reaction products, only experience will show if $\Delta n_{\text {min }}$ is attained. Although the figure of $\Delta \mathrm{n}_{\min }=9 \times 10^{\mathrm{m}} 6$ is a theoretical figure for this interferometer, it can be shown that this limit was approached when examining mutual diffusion in Iiquid systems. For instance, the average concentration difference employed was $0.06 \%$. For diethanolamine, $\triangle \mathrm{c}=0.06 \% \equiv \Delta \mathrm{n}=9 \times 1.0^{-5}$. Although the $\triangle \mathrm{n}$ is relatively high in this case, the concentration difference used was only chosen because about 8 fringe pairs (a convenient number ) were fomed at the start of diffusion. It was quite feasible to work at a lower concentration ( and hence refractive index) difference.

It had been dockdod to first apply the interferometer to the physical absorption of caxbon dioxide into water. Thus, as an illustration of the criterion of sensitivity applied to a system, the preliminary investigations will be described here. 
The only data that exists on the refractive index of $\mathrm{CO}_{2}$ in water is reported by Watson (9:1). The study made by Watson is only for dilute $\mathrm{CO}_{2}$ solutions, from whichi he gives

$$
\begin{aligned}
-4.6 \Delta n & =5.1 \times 10^{4} . c \quad \ldots \ldots \\
\text { where } \Delta n & =\left(n_{\text {water }}-n_{\text {solution }}\right) \times 1.0^{7} \\
c & =\mathrm{Co}_{2} \text { concn. } \text { mols/1itre }
\end{aligned}
$$

If this relationship is applied to $\mathrm{CO}_{2}$-saturated water $\left(c=3.31 \times 10^{-2}\right.$ g.mols/litre) then,

$$
\Delta n \approx 3 \times 10^{-5}
$$

Thus the refractive index difference should be very close to $\Lambda n_{\min }$.

A check on the refractive index of water saturatied with $\mathrm{CO}_{2}$ was attempted in this laboratory. Seven solutions were prepared by bubbling $\mathrm{CO}_{2}$ through $100 \mathrm{ml}$ water at a rate of $20 \mathrm{~cm}^{3} / \mathrm{min}$ for $14-15 \mathrm{hrs}$. The refractive indices were measured using a Pulfrich refractometer, which has: an accuracy of $\pm 0,00002$. Some difficulty was experienced with temperature control, so that one or two figures obtiained are suspect.

The refractive indices measured in this laboratory for $\mathrm{CO}_{2}$ - saturated water are listed below. Fixing the 


\section{$-203-$}

first four significant figures at 1.332 , the variation in the flfth and sixth significant figures waspo

$$
45,67,27,45,39,51 \text {, and } 45
$$

The average value for $n_{D}^{26}=1.33245$ ( refractive indices being measured at $26^{\circ} \mathrm{C}$ ).

Accepted values for the refractive index of pure water ( 92 ) are listed below:-

\begin{tabular}{l|l}
$t^{\circ} \mathrm{C}$ & $n_{D}$ \\
\hline 20 & 1.33299 \\
22 & 1.33281 \\
24 & 1.33262 \\
26 & 1.33241 \\
28 & 1.33219
\end{tabular}

It is thus evident that the refractite index difference between $\mathrm{CO}_{2}$ - saturated water and pure water is not accurately measurable by a Pulfrich refractometer, but can be expected to be of the order 10 $10^{-5}$. Thus the interferometer will be working close to Its limit.

One point which should be noted is the refractive index change of water with temperature. The change per ${ }^{\circ} \mathrm{C}$ is approximately 0.0001 . Thus using the wavefront 


$$
-204=
$$




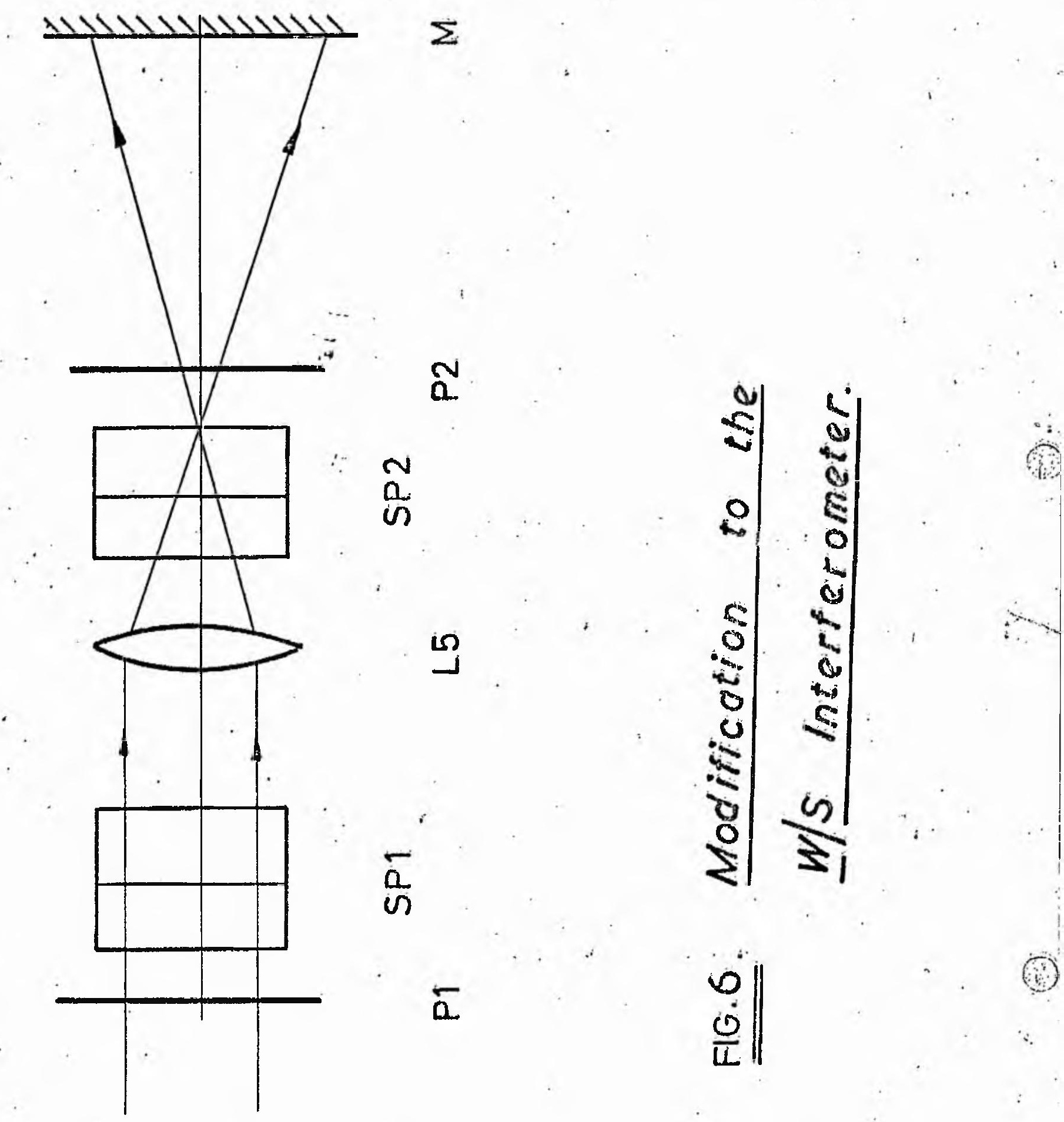


shearing interferometer (and a cell of path length $6.3 \mathrm{~cm}$ ), a temperature difference of only $0.09^{\circ} \mathrm{C}$ will be enough to produce fringes. This illustrates the importance of temperature control and the necessity of guarding against heat effects during absorption.

II(B). Description of the Apparatus.

The wavefront shearing interferometer has been fulily described in Part I of this work.

However, an important modification to the opticali arrangement, as shown in Fig.6, was made to enable a direct recording of the refractive index ( concuntration) gradient. This modification was first described by Bryngdahl and Ljunggren ( 93 ) and BxyngdahI ( 94 ). The modification consists of introducing a second Savart plate, SP2, after SPI - Polariser P2 is then put after SP2.

Strictly speaking, the gradient recorded is not in Cartesian co-ordinates $(x, y)$ but in curvilineax co-ordinates $\left(x, u_{i}\right)$, where $u=y\left(1-\frac{\phi x}{h}\right)$. Here, $\mathrm{h}=$ distance between $\mathrm{L} 5$ and $M$, and $\phi$ is given by 


$$
\phi=\frac{I}{n_{\theta}^{2}}\left(\frac{I}{n_{\theta}^{2}}-\frac{1}{n_{0}^{2}}\right)^{-\frac{1}{2}}
$$

where $n_{e}$ and $n_{0}$ are the principel refractive indices of the Savart plate.

In the system used in this laboratory, $h=600 \mathrm{~mm}$, and $\phi=0.3$. To aIl intents and purposes, therefore, the gradients recorded by the interferometer are in Cartesian comordinates.

The Gas-Liquid Cell is diagramatically shown in Fig.7, and a photograph (Fig.8) shows the external appearance.

The cell is constructed throughout of stainless steel, and the dimensions are based on the flowing junction cell which was used successfully in the liquid-liquid diffusion experiments. The optical path length through the cell is $6.3 \mathrm{~cm}$. , and the interfacial area between the gas and the liquid is $32 \mathrm{~cm}^{2}$. Optical glass flats, $10 \mathrm{~mm}$ thick, are clamped paralilel to the precision machined faces of the cell. by 12 spring held screws. Coppex and rubber gaskets ( $1 / 16^{\prime \prime}$ and 2 thick respectively) distribute the screw pressure. Gas is admitted through one of the ports $\left(3 / 16^{\prime \prime}\right.$ a.dia.) and is deflected by the baffle. Distribution over the liquid surface is aided by the stainless steel gauze (200 


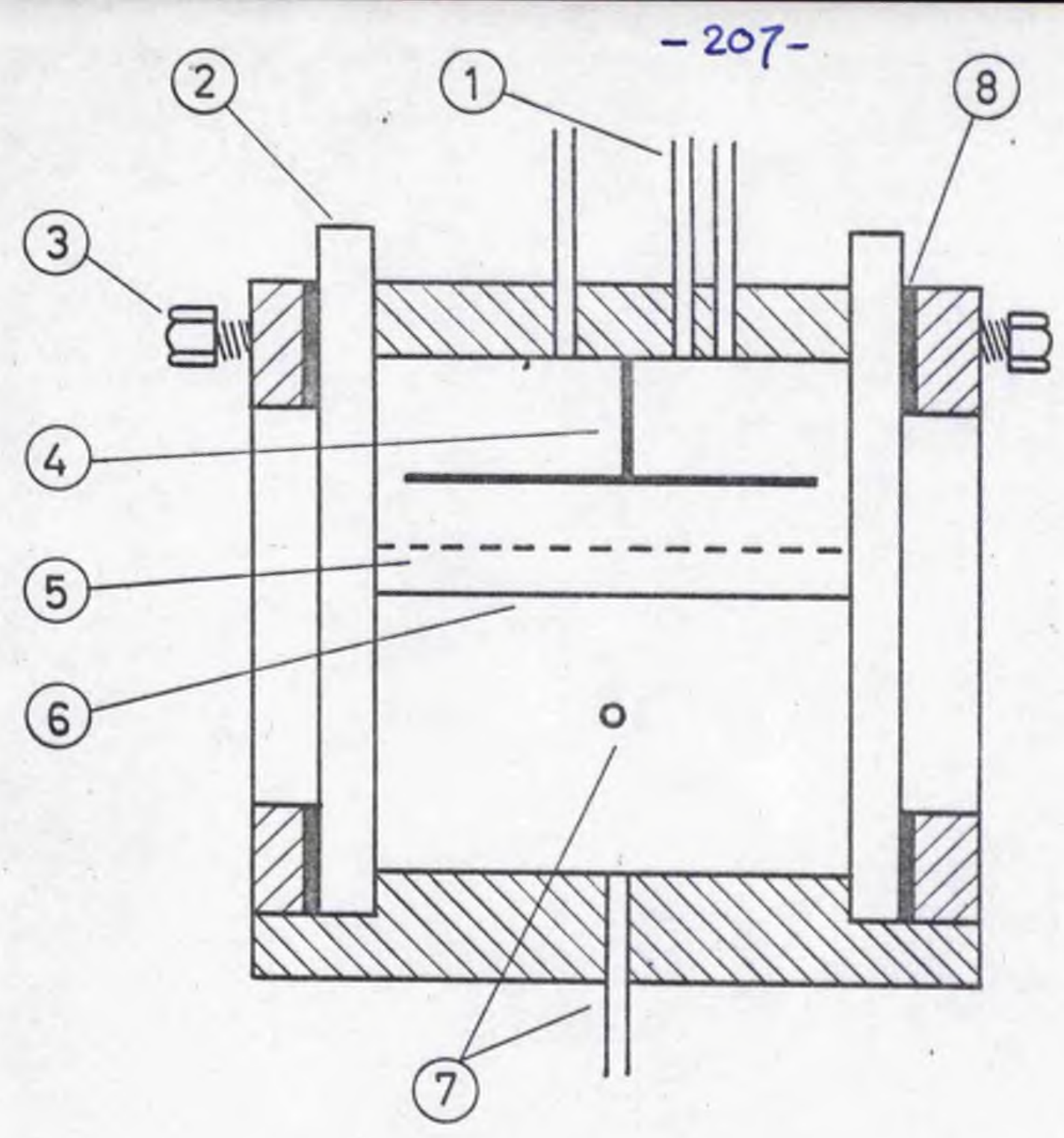

(1) Gas Ports

(5) Gauze

(2) Optical Flats

(6) Knife-Edge

(3) 12 Screws

(7) Liquid Ports

(4) Baffle

(8) Copper/Rubber Gaskets

FIG. 7. The Gas-Liquid Cell. 


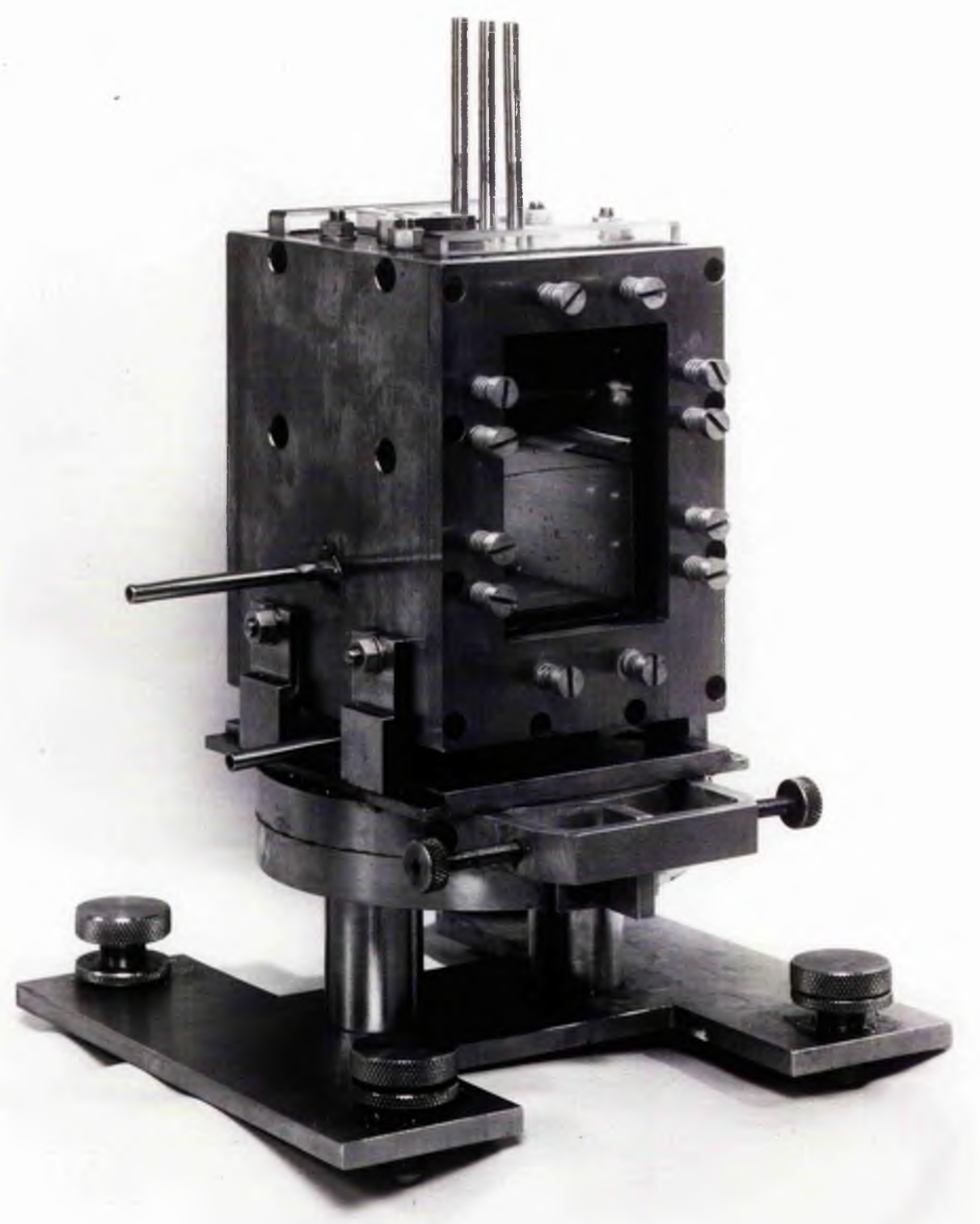

Eig.8. The Gasmiquid Cell. 


$$
-209-
$$




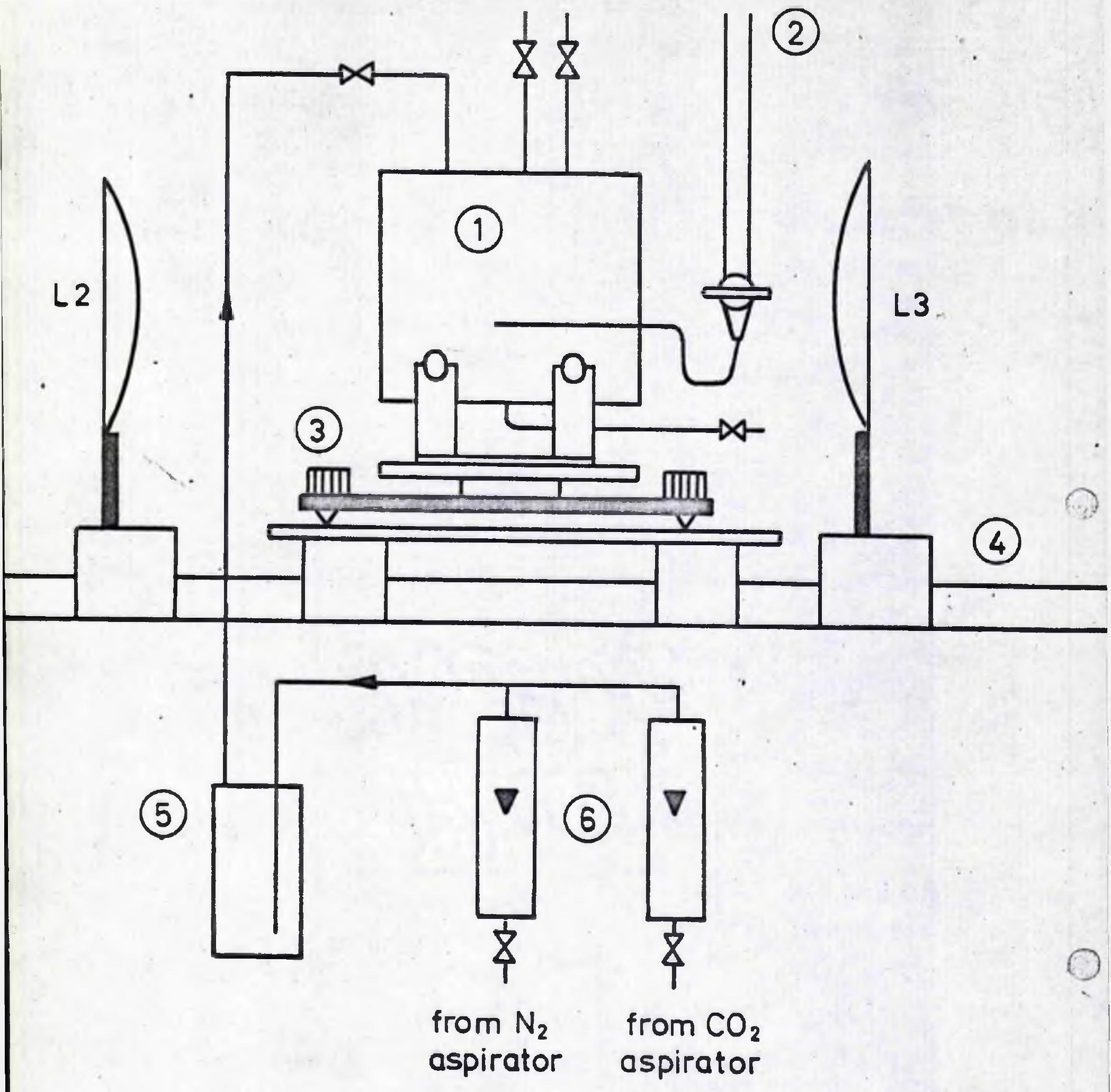
(1) Gas Cell
(4) Optical Bench
(2) Burette
(5) Wash Bottle
(3) Cell Base
(6) Gas Rotameters

FIG.9. Arrongement of $\mathrm{Cell}$ on Bench 
mesh/inch ).

The Iiquid is admitted via, a burette, so that a careful control of the liquid level can be effected. This is necessary, since an ingenious device has been incoxporated into the cell to eliminate the meniscus (thus making Identification of the interface more accurate). This device consists of a silver knife-edge, "/1000" thick, projecting I. mm round the walls of the cell. The engineering of this knife-edge is a matter of some delicacy, but once: accomplished the result is highly successful.

The cell is mounted on a circular base, attached in turn to an H-shaped base with four adjustable screws in the legs. The circular base may be rotated by means of a screw device, so that the axis of the cell may be aligned with the optic axis.

The admission of gas to the cell for contacting with a solution is in itself a technique ( since it is so essential to minimise all heat effects ).

The first arrangement tried for admitting gas to the cell is shown in Fig.9. Two 5-litre aspirators contained $\mathrm{CO}_{2}$ and nitrogen, - the $\mathrm{CO}_{2}$ being maintained over a concentrated magnesium sulphate solution. The gas ( concentrated, or diluted with nitrogen) is metered through 
two rotameters ( ronge $0-25 \mathrm{ml} / \mathrm{min}$ ) and passed through a. sintered, glass disc (porosity 1. ) wash bottle。 This permits a visual check on the gas. rate and ensures additionali saturation of the gas. Howevex, this arrangement is not veryt successful, since the passage of gas over the liquid introduces eithex evaporation or condensation, - heat effects being apparent by the formation of fringes even when pure nitrogen is passed over.

A second arrangement which is almost entirely successful is shown in Fig. IO. One of the cell gas ports Is connected to the $\mathrm{CO}_{2}$ aspirator, and the other to the $\mathrm{N}_{2}$ aspirator. Tap B is closed, so that the cell is under a slight pressure equal. to that in the $\mathrm{N}_{2}$ aspirator, which in turen is equal to that in the $\mathrm{CO}_{2}$ aspirator (approx. 8. mm Hg. gauge). Before contacting the gas with the liquid, the $\mathrm{N}_{2}$ aspirator gas jar is elevated about $7 \mathrm{~cm}$ ( thus introducing a pressure difference between the $\mathrm{CO}_{2}$ and $\mathrm{N}_{2}$ aspirators of about $7 \mathrm{~cm}$ water ). Shortly afterwards, tap $\mathrm{B}$ is opened. $\mathrm{CO}_{2}$ is thus brought into contact with the Iiquid whilst equalising the pressure in the system. Negligible physical changes are introduced by this method. However, a disadvantage of the method is that gas concentrations are not easily controllable. A proposed 


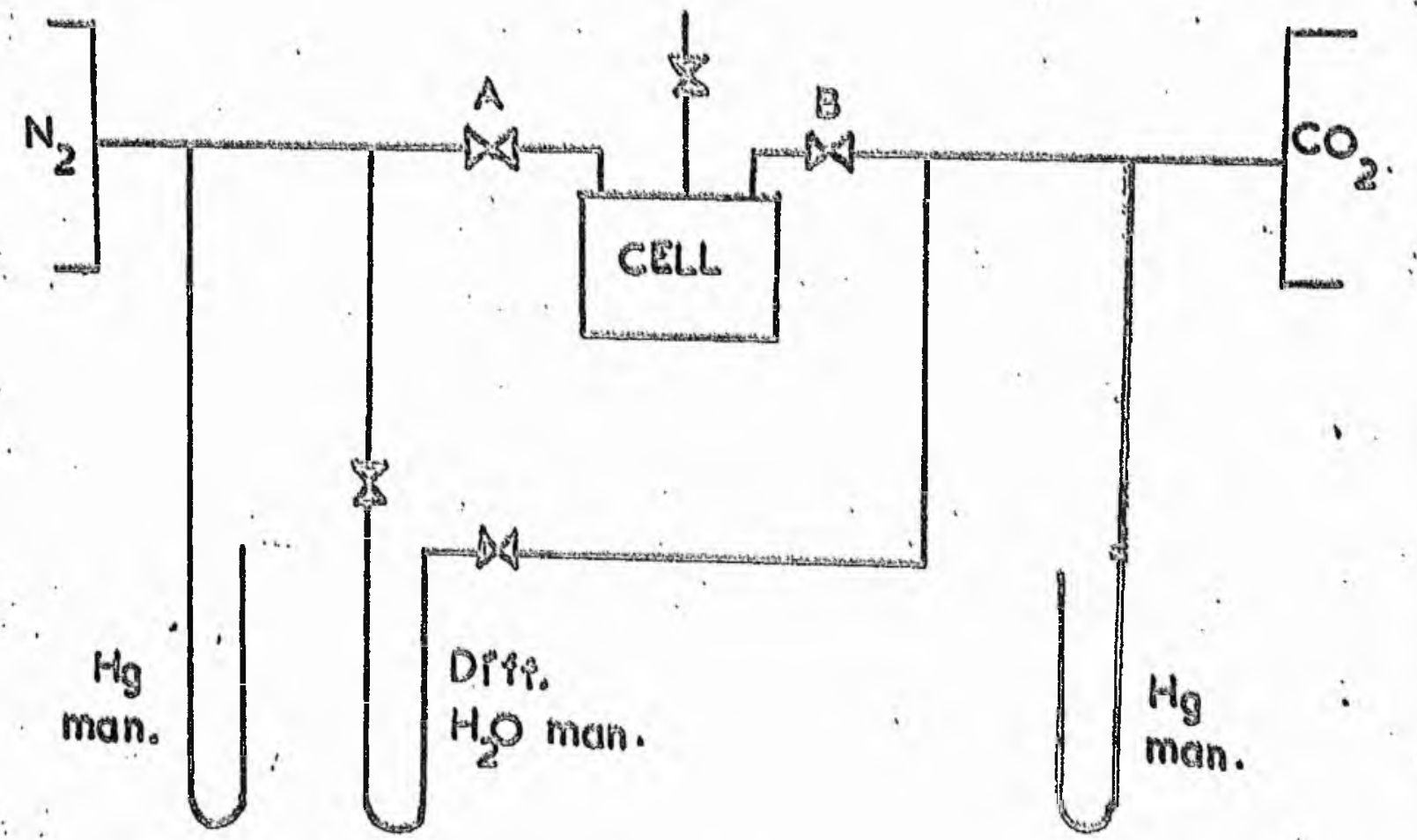

F10.10.

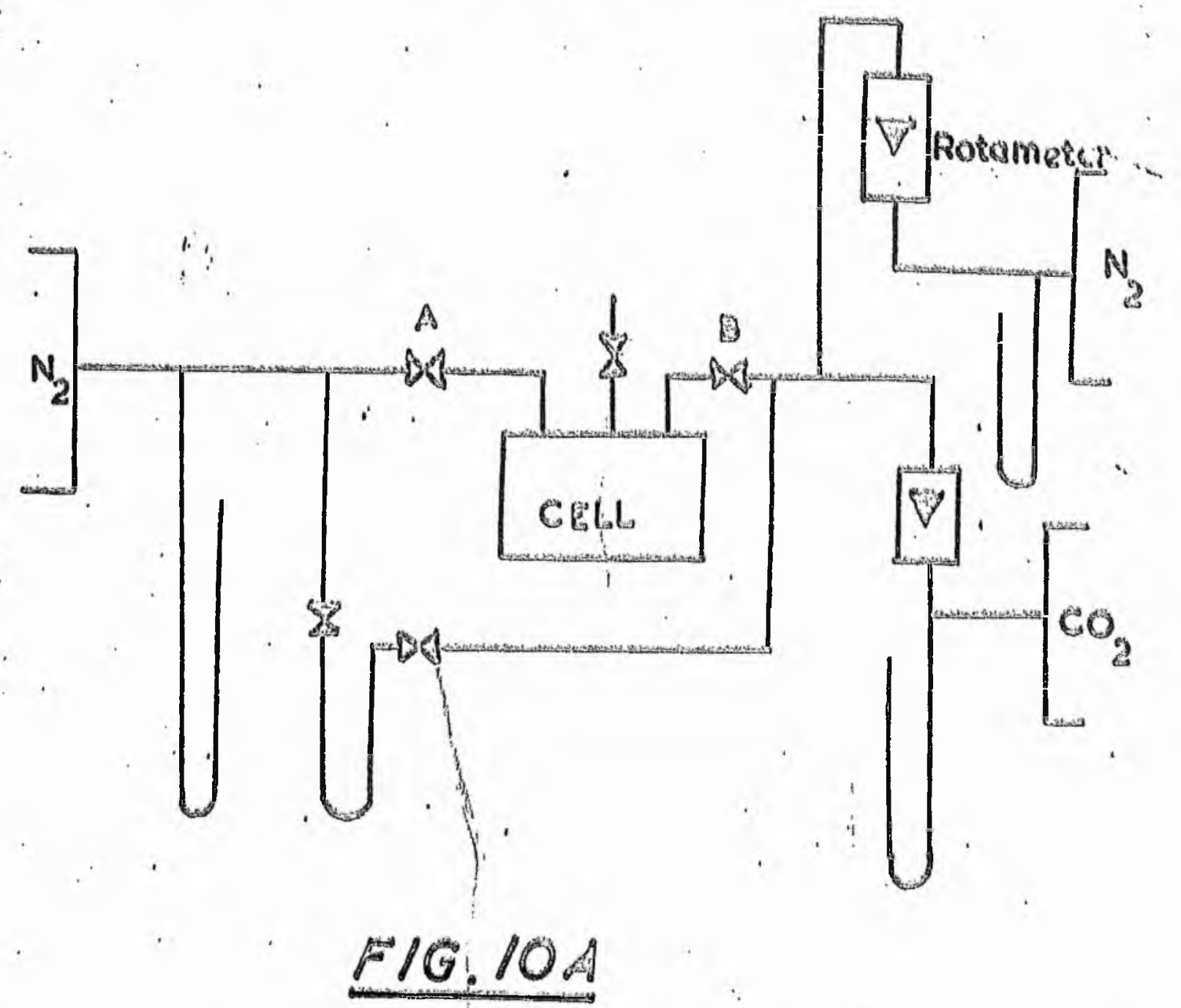




\section{$-213-$}

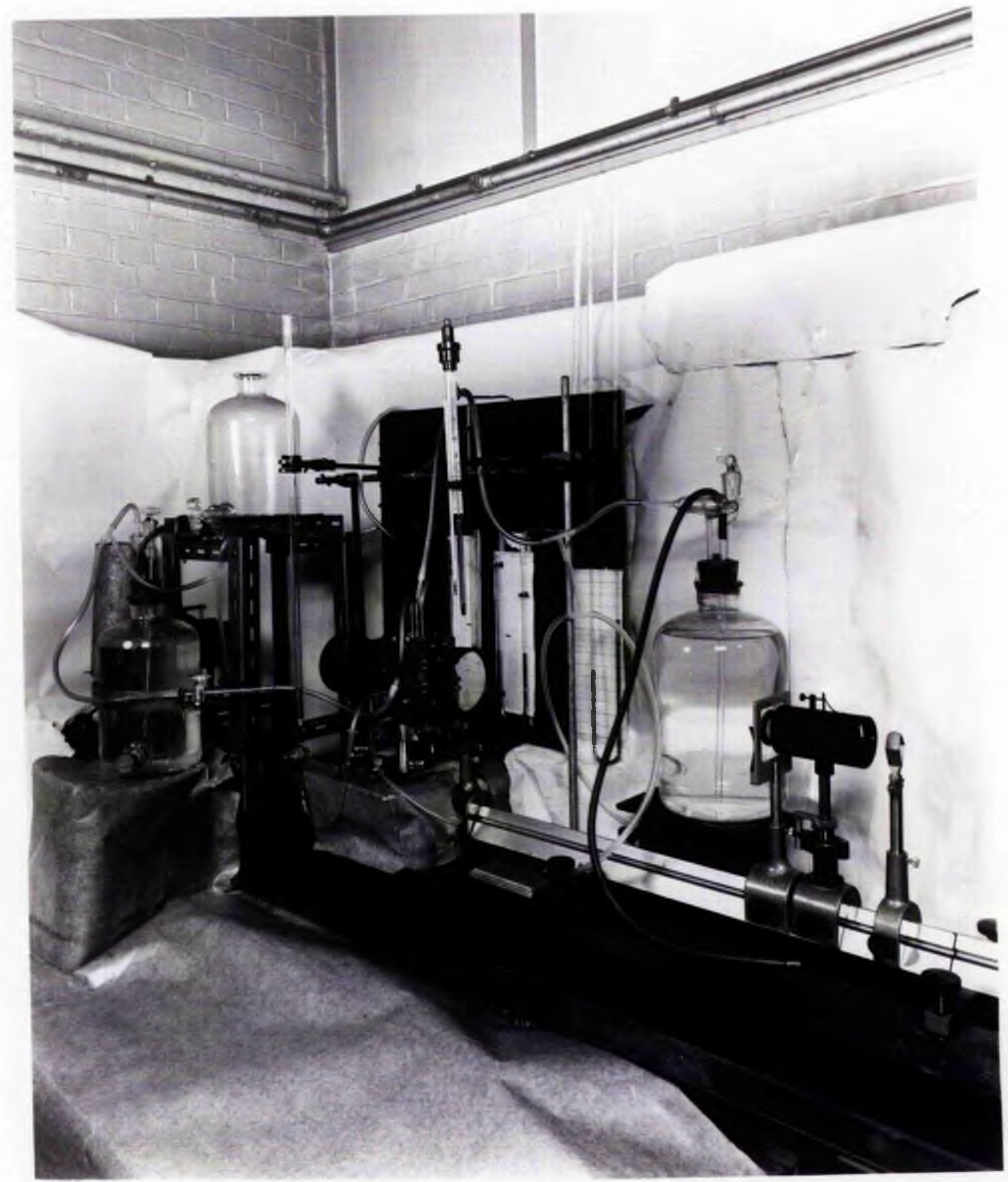

Fig.1I. General VLew of Optical Bench \& Gas Cell. 


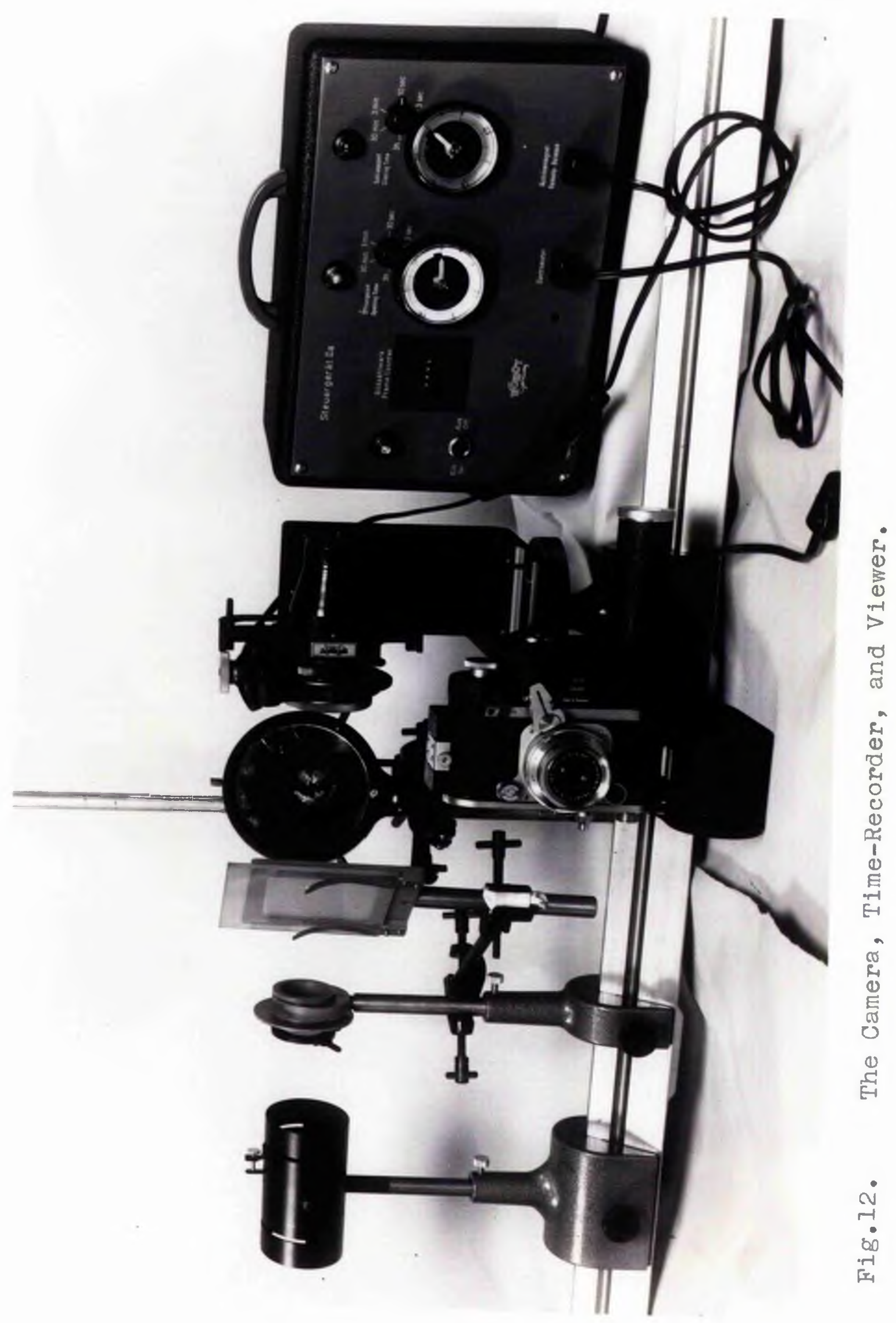


modification is to introduce a third aspirator as in Fig. IOA, with careful metering of the gas flows

The optical bench, with the gas cell fitted up as In Fig.10, is show in the photograph in Fig. II. The camera, time recorder, and viewer are shown in Fig.12. It will be noticed in Fig.12 that in front of the viewer, in place of which the camera is mounted during runs, is a hinged mirror and ground glass screen. This is for inspection of the image during photographic registration.

\section{II(c). Experimental Technique.}

The gas. cell is thoroughly cleaned, taking care not to damage the knife-edge, before the glass flats are screwed into position and the whole unit is centared an the optical bench. The cell is purged with nitrogen. - Using the burette, the cell is filled with the: solution to be contacted with the gas. As explained. 
previously, the cell is filled via. the buretite so that the liquid level can be controlled. If this is done, it is simple to reduce the meniscus to a very fine depth by means of the knife-edge. Thus, identification of the interface is facilitated.

The gas cell is connected up to the aspirators, which have been filled with their respective gases, as in Fig.10. The aspirators are at: equal pressres. The complete unit is then left overnight in the thermostatically controlled constant temperature room (maintained at $25 \pm 0.1^{\circ} \mathrm{C}$ ).

When about to start a run, the: Robot Recorder camera is loaded with Kodak R. 55 recording film, and the timing unit is set for I sec. exposures every 4 seconds. The 250w mercury vapour lamp is started up, and after Inspection of the image ( to verify that the fringes are ali vertical, - any bending indicates preliminary mass transfer ox thermal imbalances ) the camera is placed in position.

The gas jar of the nitrogen aspirator is raised about $7 \mathrm{~cm} .$, - thus effectively lowering the pressure in the gas cell and aspirator by a.bout $7 \mathrm{~cm}$. water. Tap $\mathrm{B}_{9}$ from the carbon dioxide aspirator, is opened, and at this 
instant the timing of the photographic ragistration is started. Aftex about 60 seconds the image is inspected (using the hinged mirror and ground glass screen). With absorption of $\mathrm{Co}_{2}$ into dilute monoethanolamine, it is quite possible that by this time cumulative heat effects will be disturbing the solution sufficiently to make further photographic registration unneccessary ( this will be illustrated later on). However, if careful control of concentrations is effected, it may be possible to eliminate these: heat effects and to continue photographic registration for a considerable time further. With physical absorption these heat effects are not apparent, and inspection and recording of the fringes continues until the fringes are nearly vertical.

The exposed R. 55 recording film is developed in Kodak DX.80 developer for 2 minutes at $25^{\circ} \mathrm{C}$.

Measurement of the concentration profiles is effected by projecting the photographic: inage.onto a frosted screen (at a magnification of about $60 \mathrm{x}$ ), and tracing the profizes or reaction zone position. It does not appear that a recording microdensitometex can be applied in this case. 


\section{EXPERTIMENTAL RESULTS.}

III(a). Physical Absorption of $\mathrm{CO}_{2}$ into water.

Several repeated runs were made for the diffusion of $\mathrm{CO}_{2}$ into water, and a photograph of a typical refractive index ( concentration) gradient recording from one of the runs is shown in Fig.13. The exposure shown is for $t=720$ seconds.

In tabile $I$, measurements for concentration gradients recorded at $t=205$ and $t=720$ seconds for one run are. given. Also tabulated are the values of the diffusion coefficient which would satisfy the curves at each point.

In Fig. 14, the theoretical concentration profiles for the diffusion of $\mathrm{CO}_{2}$ into water are given. These have been calculated for $t=20,90$, and 720 seconds, using equation 4. Values of exfe $x$ have been computed and tabulated by the author, between $x=0.0000$ and $x=2.3000$ (at intervals of 0.0001 ). The algebraic expansion

$$
\operatorname{erfc} x=\frac{2}{\sqrt{\pi}} \int_{x}^{\infty} e^{-t^{2}} \cdot d t
$$




$$
\text { or, } \quad \operatorname{erfc} x=1-\frac{2}{\sqrt{\pi}}\left[x-\frac{(-1)^{n} \cdot x^{2 n-1}}{(n-1) !(2 n-1)}\right]
$$

was used, where $n$ is the number of terms in the series expansion. The series was expanded as follows;-

$\begin{array}{ccc}x & \text { no. of terms } \\ 0 \text { to } 1 & 9 \\ 1 " 11.2 & 14 \\ 1.2 " 11.4 & 16 \\ 1.4 " 1.6 & 18 \\ 1.6 " 1.8 & 20 \\ 1.8 " 2.0 & 24 \\ 2.0 " 2.3 & 32\end{array}$

A value of $D=19.5 \times 10^{-6} \mathrm{~cm}^{2} / \mathrm{sec}$ for the dirfusion coefficient of $\mathrm{CO}_{2}$ into water ( 80$)(95)$ was taken in computing the theoretical concentration gradients.

The experimental concentration gradient for $t=$ 720 secs has been plotted together with the theoreticel curves for comparison.

From table $I$, and also from a comparison of the theoretical and experimental concentration profiles in Fig.I4, it is apparent that the experimental diffusivity 


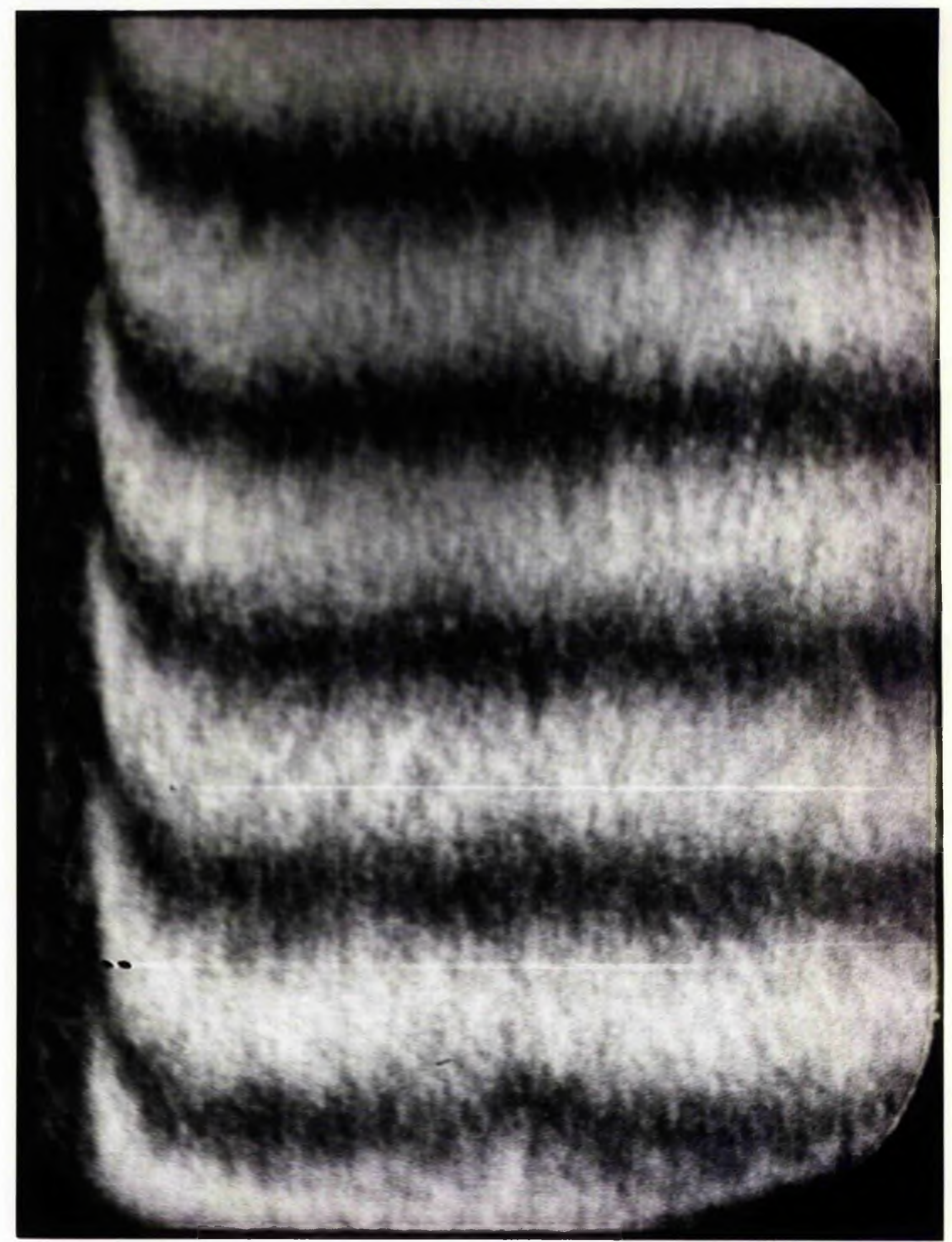

Pig.13. $\mathrm{CO}_{2}$ - water refractive index gradient recording. 


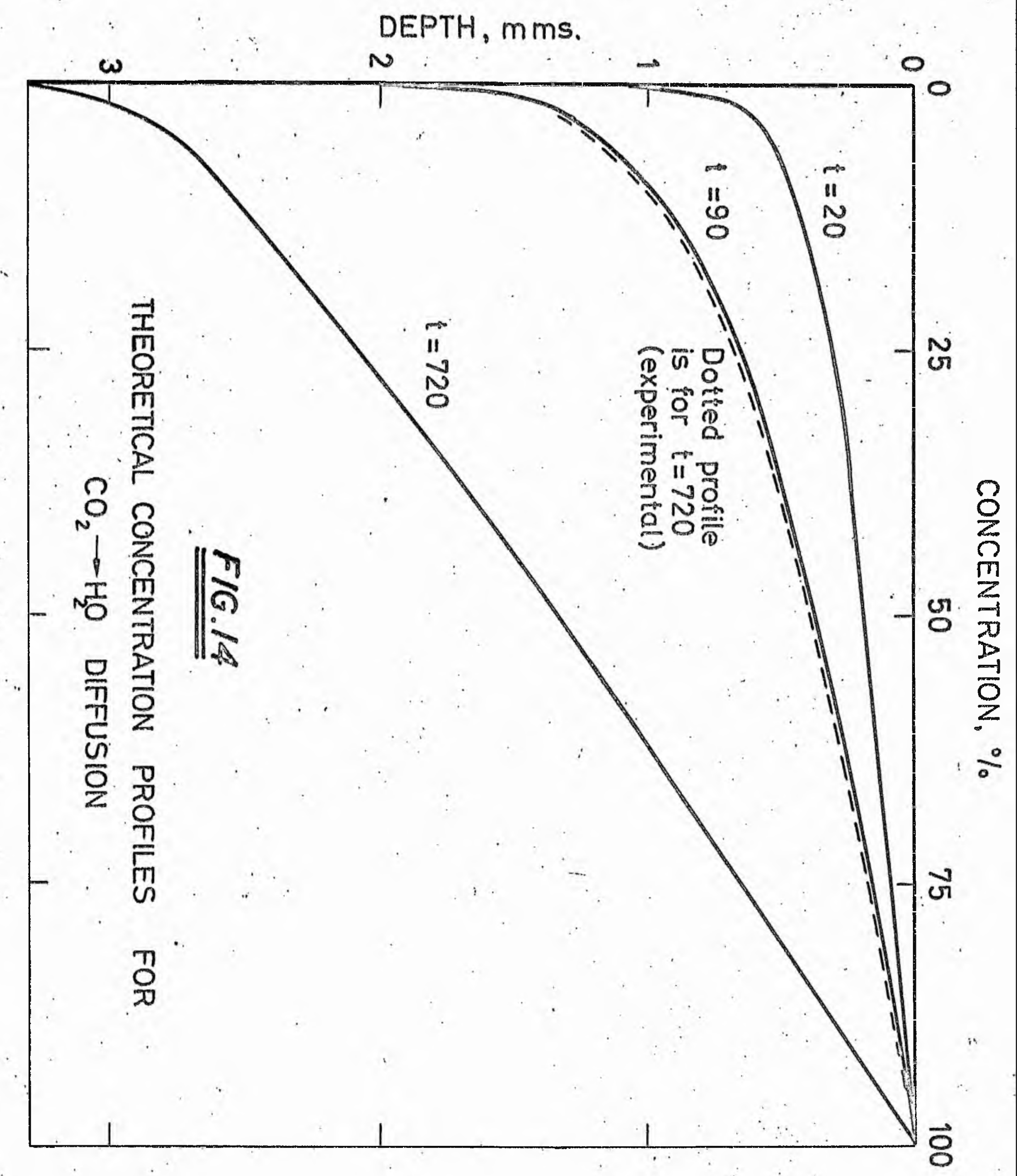




\section{$-222 m$}

Table 1. Experimental Diffusion of $\mathrm{CO}_{2}$ into water.

Concentration Gradients: recoxded.

$G=55.8$

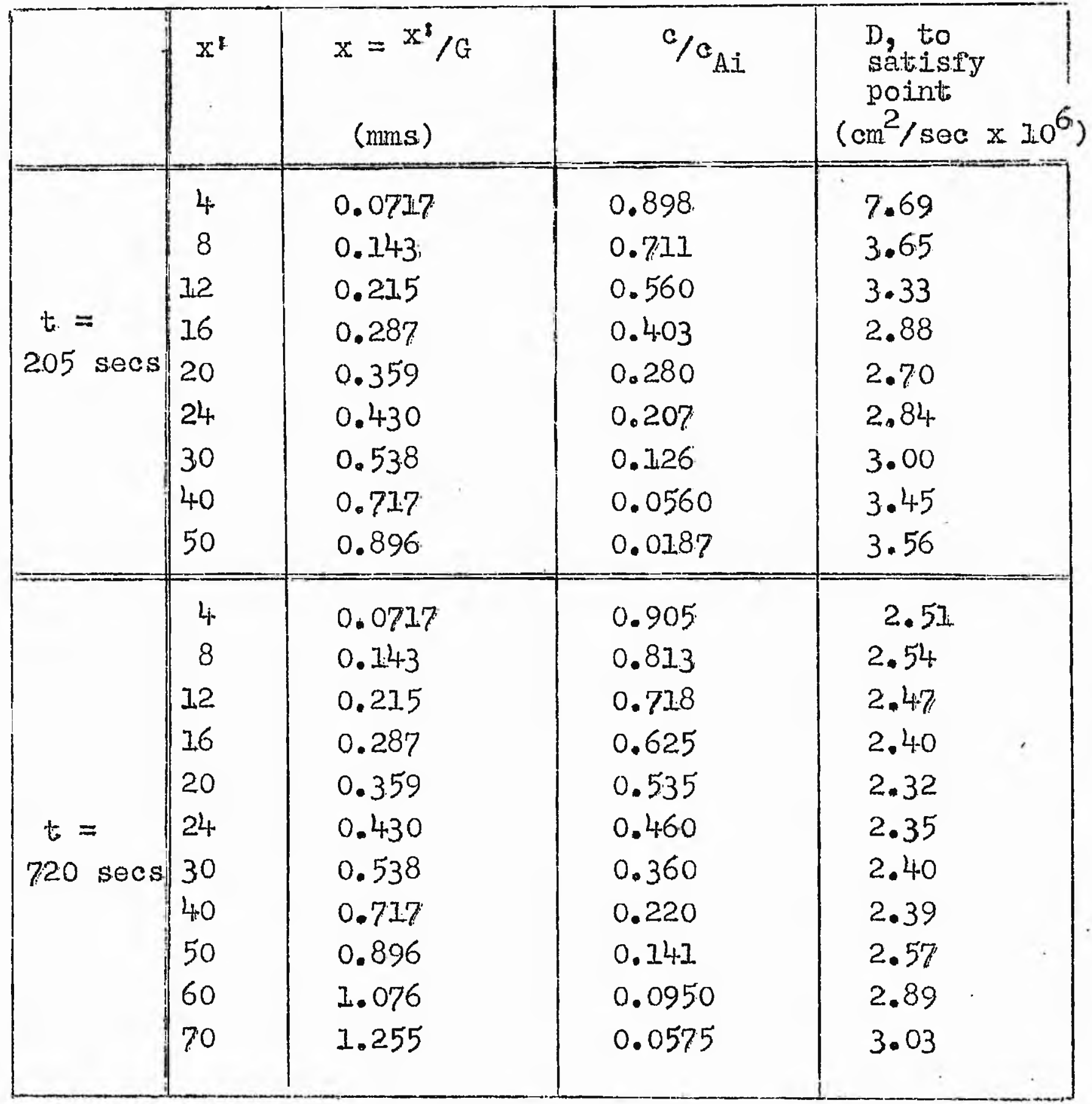


of $\mathrm{CO}_{2}$ into water is too low by a factor of about 8 , and as such is unreal. It must be emphasised here that these runs were repeated many times, and the recorded concentration profiles were all similar in their apparent $D$ values to the two profiles reproduced in table 1.

The main conclusion that may be postulated from these repeated failures at measuring the correct diffusion coefficient, is that the interferometer is working at its absolute limit of sensitivity. This is supported by an examination of the recorded concentration profiles.

In Fig.I3 it will be notice that the maximum "bending" of the gradients is only equal tol or slightly less than ) the equidistant spacing apart of the fringes. Since this spacing is equivalent to an optical path length of $\lambda / a(4)$, it would appear that the refractive index difference between $\mathrm{CO}_{2}$-saturated water and pure water is only about $9 \times 10^{-6}$.

(In the discussion on the sensitivity of the interferometer, it will be remembered that the refractive index difference was expected to be of the order 10-5.)

Since the refractive index difference is so apparently low, then there is a good possibility that distortion of the recorded gradients will occur. It may 
well be, then, that there is not a sufficient refractive index difference present initially to enable any accurate measurements to be made of the diffusion of $\mathrm{CO}_{2}$ into water. If this is so, then remexamination and remppraisal of previous workers'results using interferometers to study diffusion of $\mathrm{CO}_{2}$ into water will be necessary.

Harvey and Smith ( 46 ) used a Mach Zender interferometer to determine the interfacial resistance in a liquid film to the absorption of $\mathrm{CO}_{2}$. Nowg since a Mach Zender is a good deal less sensitive than a wavefront shearing interferometer,$\Delta n_{\min }=6 \times 10^{-4}$ being quoted by Mullin et al ( 24 ), then it is unlikely that their : observed concentration gradients were true mass transfer gradients. Also, a feature of Harvey and Smith's experimental. technique was the sudden changing in pressure of the system from vacuum to atmospheric. Since this will undoubtedly have caused marked physical changes in the system and introduced large heat effects, their technique and results need very careful reconsideration.

Recentiy, Jepsen et. al ( 96 ) reported on the offect of wave-induced turbulence on the rate of absorption of gases in falling liquid flims. One of the basic features of this work was the use of a Mach Zendex interferometer 
to determine concentration profiles for the diffusion of carbon dioxide Into water films. Although Jepsen describes the close temperature control of the water he uses, there is no account of any attempt to control the temperature and humidity of the gases. Since Jepsen used a flowing system, then it is quite probable that thermal inbalances were introduced, so that, again, it is quite probable that the gradients observed were not true mass transfer gradients.

Although the wavefront shearing interferometer has not produced any accurate profiles for the physical absorption of $\mathrm{CO}_{2}$ into water, there is no reason why itt should not yield true gradients where a more soluble gas j.s concerned.

III(b). Absorption of $\mathrm{CO}_{2}$ into dilute Monoethanolamine.

Using a I g/liftre MEA solution $(0.0166 \mathrm{~N})$ several runs were repeated for the absorption of $\mathrm{CO}_{2}$ into dilute monoethanolamine solution. Other concentrations of MEA were used, but due to an initial lack of experimental technique these runs were of little value. only when experience had been gained, and the interpretation of the 
$-226=$ 


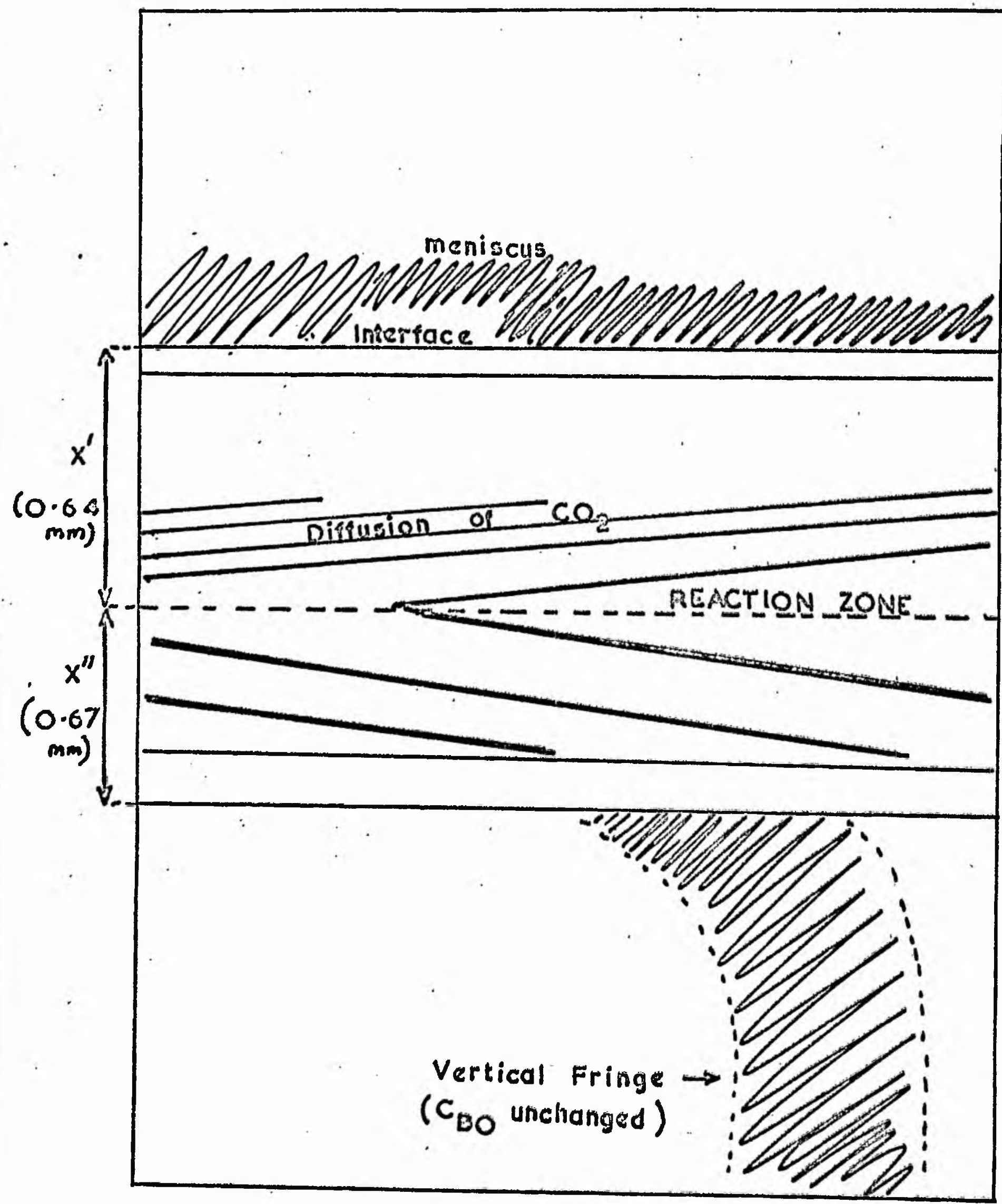

FIG.15 Diogramanic Derail of Fig. 16 


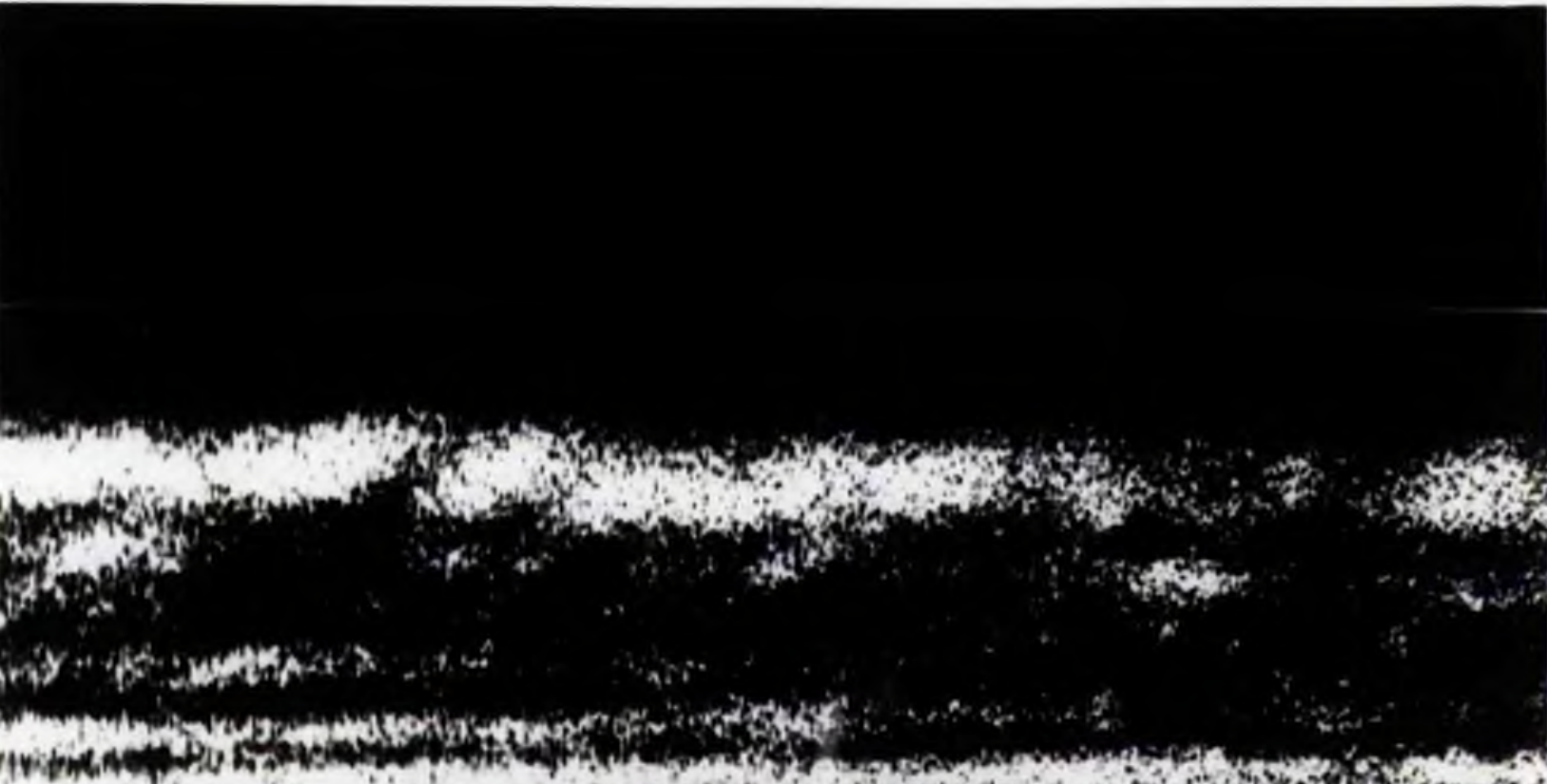

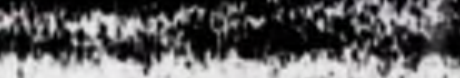

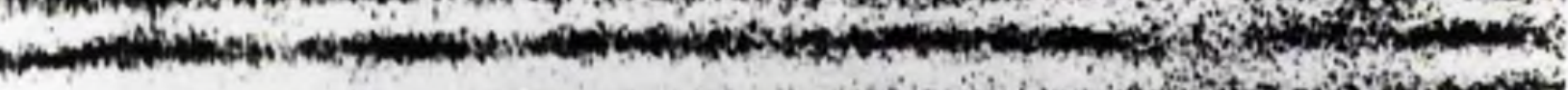

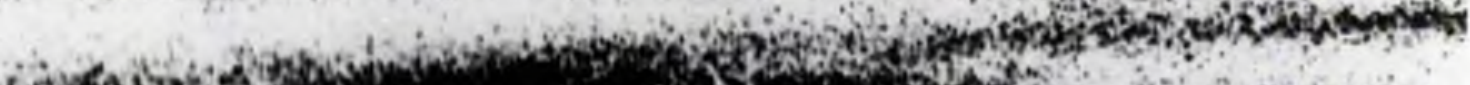

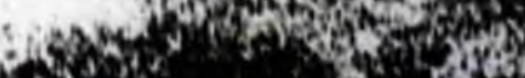

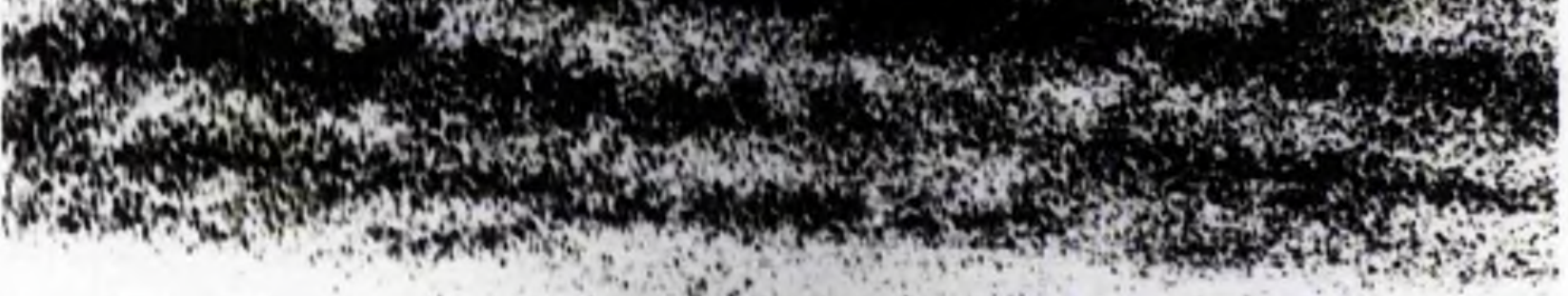

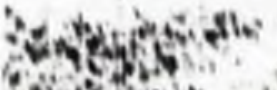

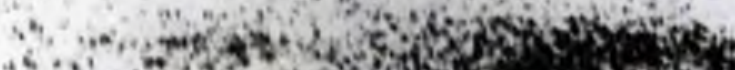

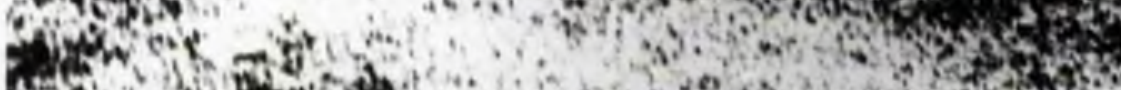

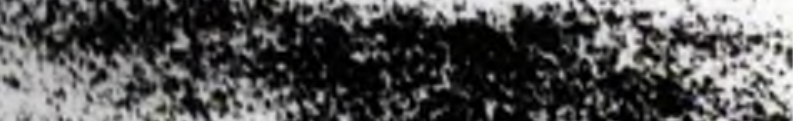

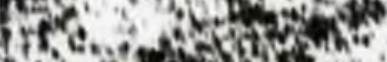

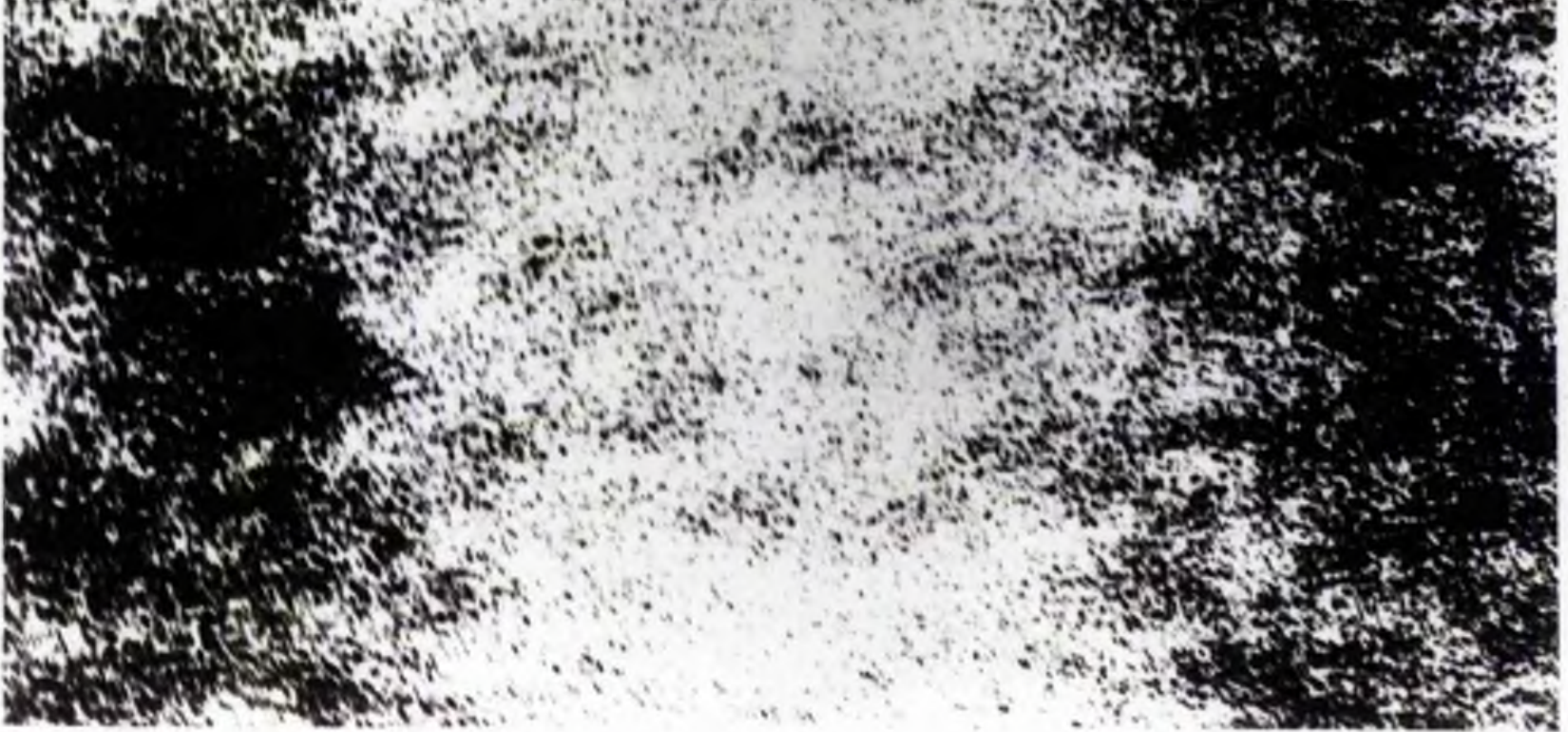

FIG. 16

CONCENTRATION PROFILES OBSERVED DURING ABSORPPION OF $\mathrm{CO}_{2}$ BY 0.0166 N MEA . 
photographic recordings was accomplished, did any consistency appear in the results. Thus, only two runs, both using $0.0166 \mathrm{~N} \mathrm{MEA}$, are recorded here.

It will be remembered from the theory of absorption accompanied by a fast 2 nd order irreversible reaction, that a reaction zone is formed in such a case,- the reaction zone moving away from the interface. In Fig. 16 a detall of the photographic recoraing of this reaction zone is shown for $\mathrm{CO}_{2}$ diffusing into $0.0166 \mathrm{~N} \mathrm{MEA}$ after 57 seconds. In Fig. I5 the main features of this photograph are drawn, so that the principal regions and dimensions may be identified. Note the scale of the photograph (approx. 60x mag. factor). These features will now be described; -

I) The top, totally dark, region is the meniscus. (In this photograph, as well as in Figs. I7A to D, a deep meniscus is apparent. This is due to the knifemedge not being used, having been damaged earliex on. The presence of a meniscus does not in any way prejudice the results)

2) Beneath this dark region is an apparent narrow white band. The top of this band has been identified as the interface.

3) Beneath the interface, a set of lines run 
slightly downwards from right to left. These lines represent the steep concentration gradient of $\mathrm{CO}_{2}$ diffusing through the reaction products solution.

4) Meeting the $\mathrm{CO}_{2}$-diffusion gradient lines are a set of lines sloping upward from left to right. These Ines have been taken to represent the concentration gradient of MEA diffusing upwards ( and possibly also the downward diffusion of the reaction products).

5) Where the two sets of lines meet, the peaction zone is assumed to lie.

6) The lower edge of the Lower white band is taken as the extremity of the liquid film.

The above interpretations of the photographic recordings of the absorption of $\mathrm{CO}_{2}$ by dilute $\mathrm{MEA}$, taken using the wavefront shearing interferometer, is tentatived It must be remembered that this is the first time that the presence of the reaction zone, in absorption with fast and order reaction, has been illustrated. Previously an entirely theoretical concept, it is now possible to actually observe this reaction zone and its movement.

A series of photographs illustrating the movement of this reaction zone are shown in Figs. 17A to $D$. 
$-230$

Fings. IIA to I7/D.

Photographs showing movement of the Reaction Zone in absarption of $\mathrm{CO}_{2}$ by $0.0166 \mathrm{~N} \mathrm{MEA}$. The times of exposure were as follows;

$3 \operatorname{secs}(A) ; 21 \operatorname{secs}(B) ; 33 \operatorname{secs}(C) ; 51 \operatorname{secs}(D)$. 


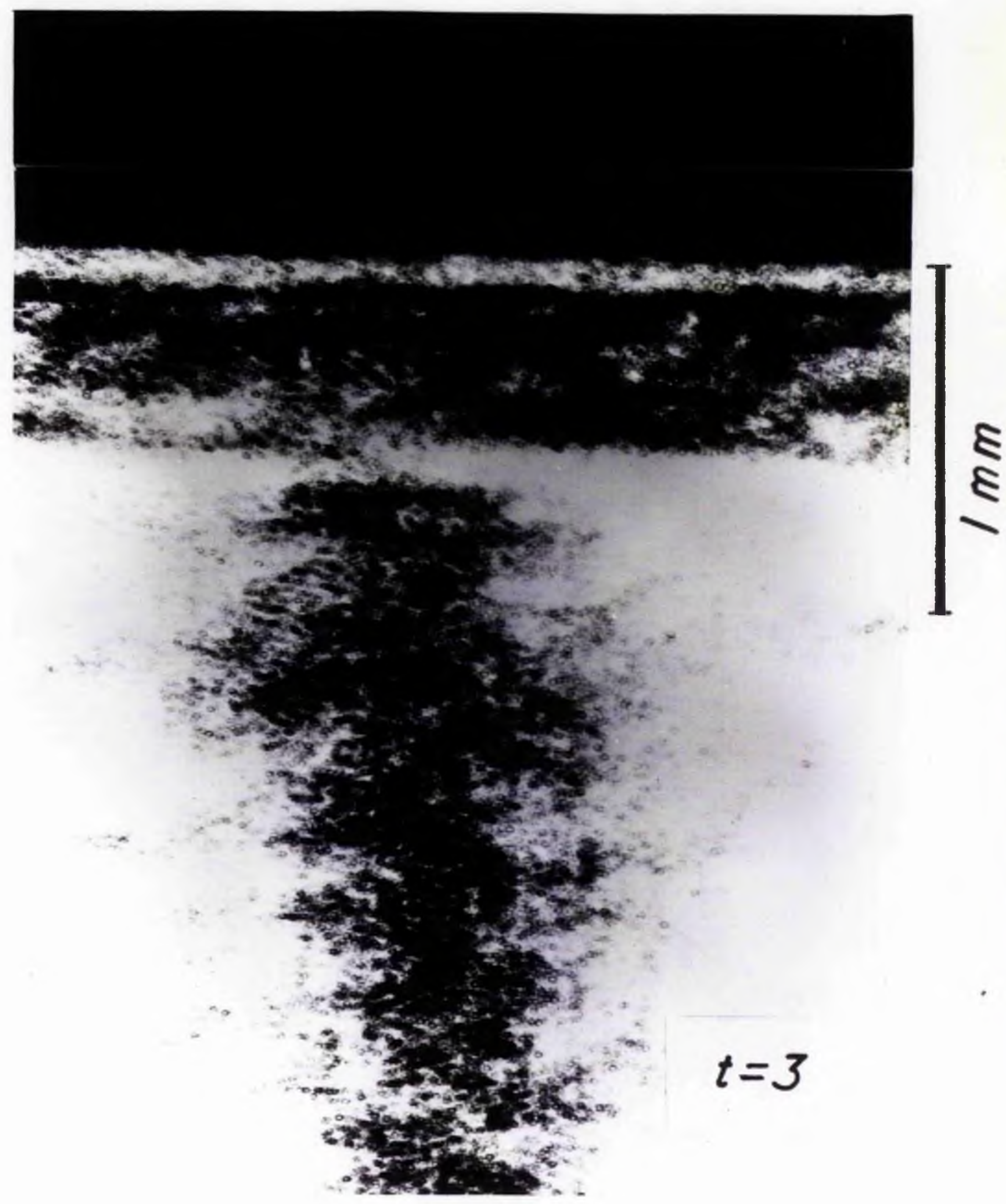



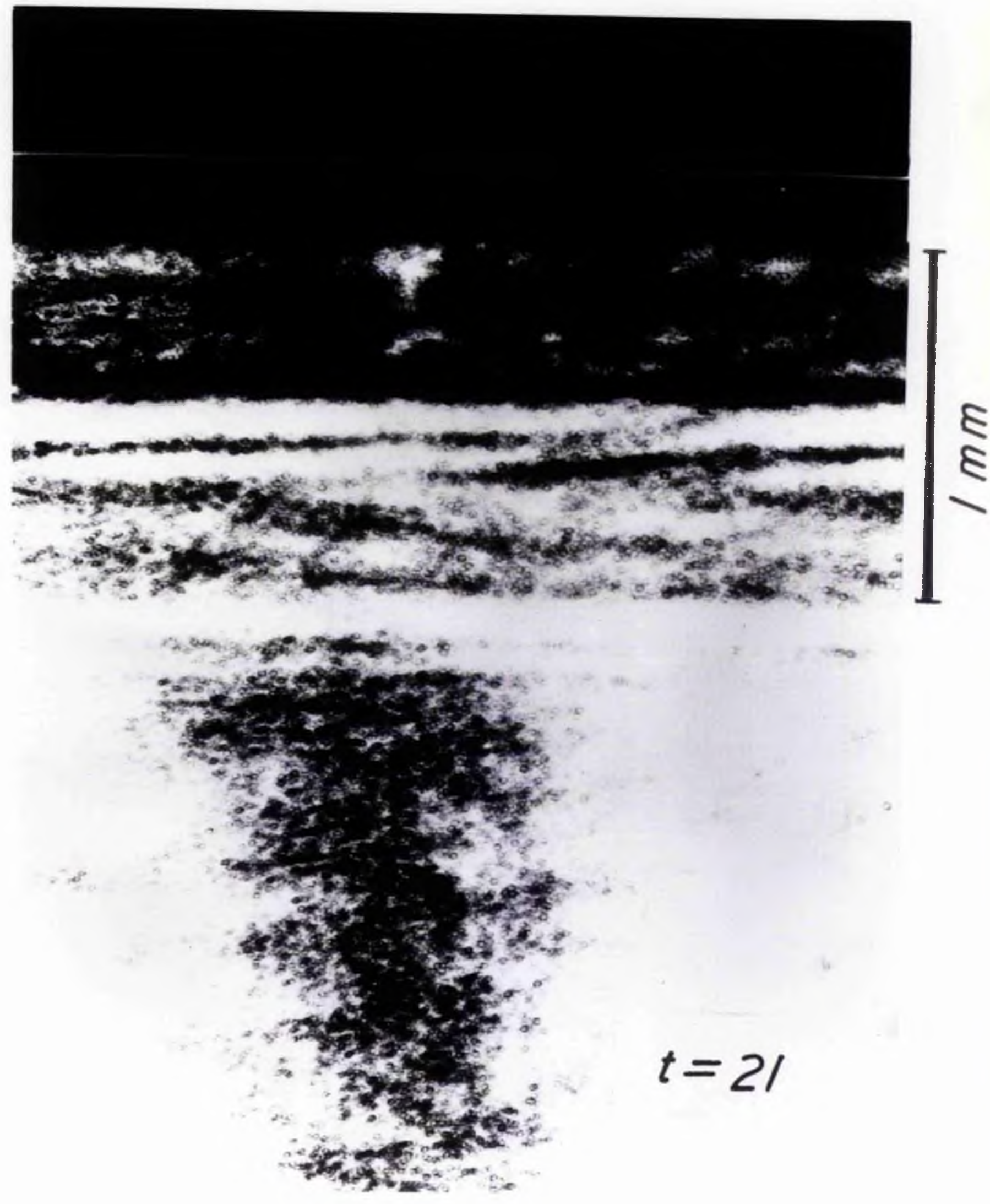

FIG. 17B 


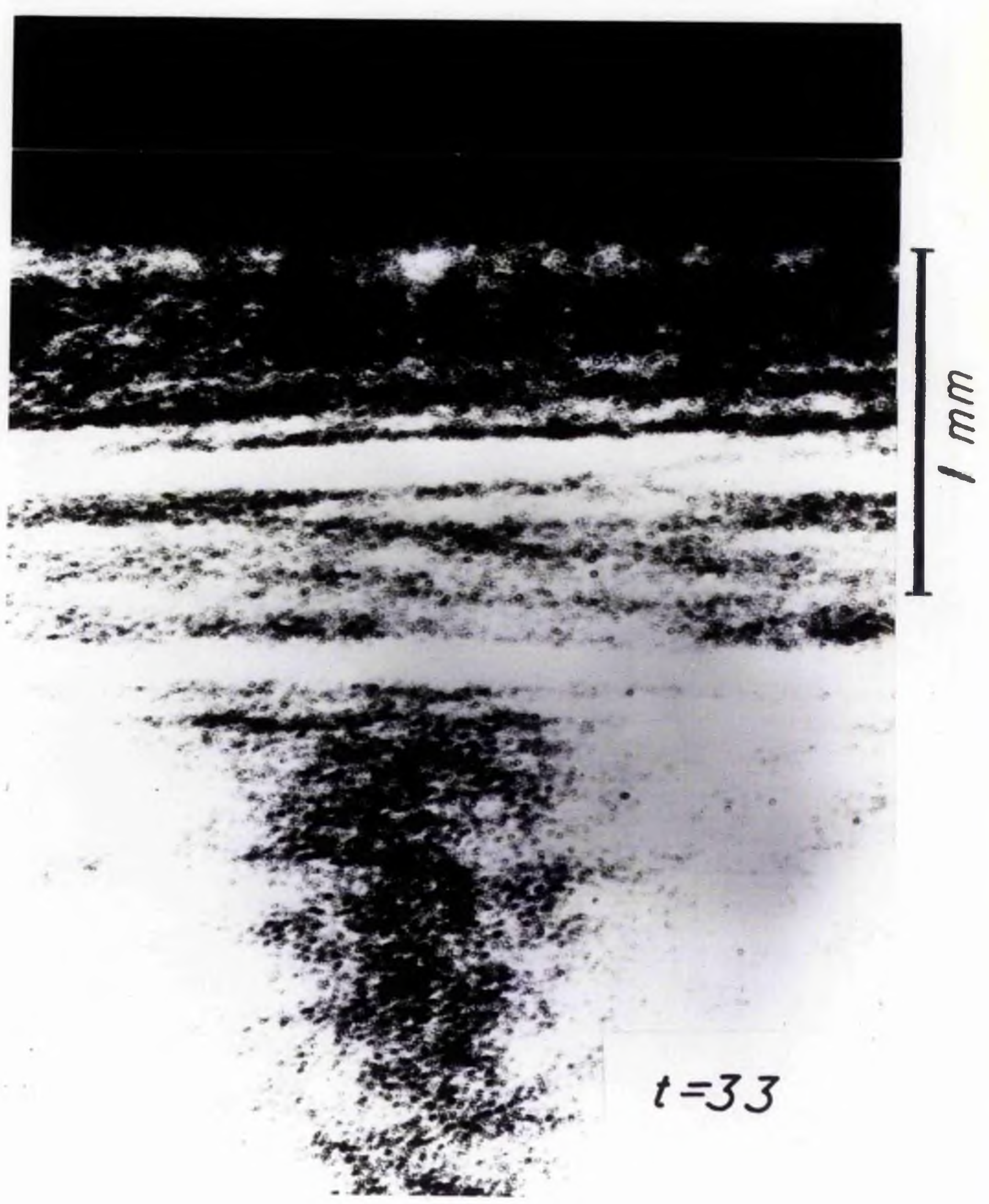




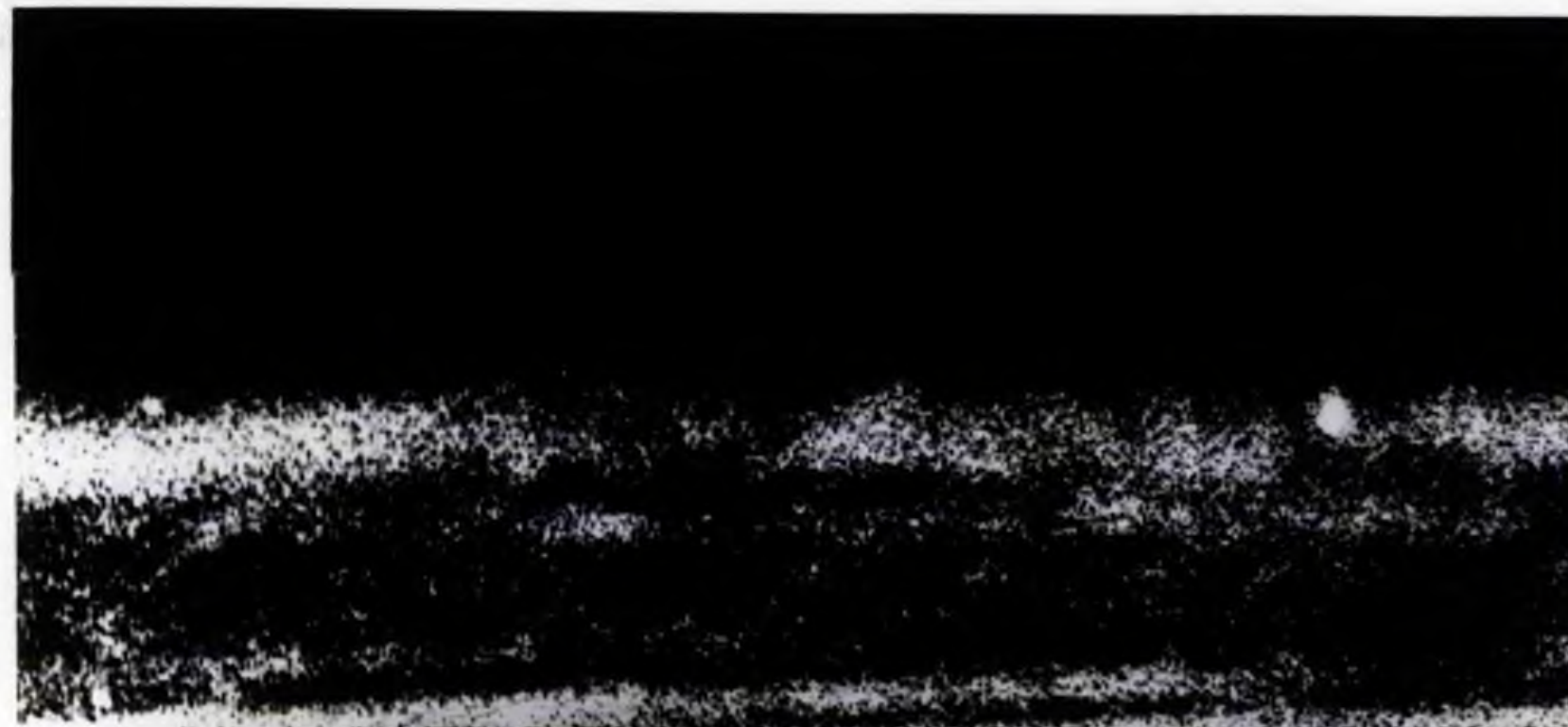

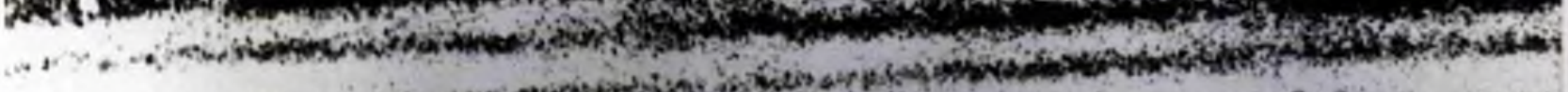

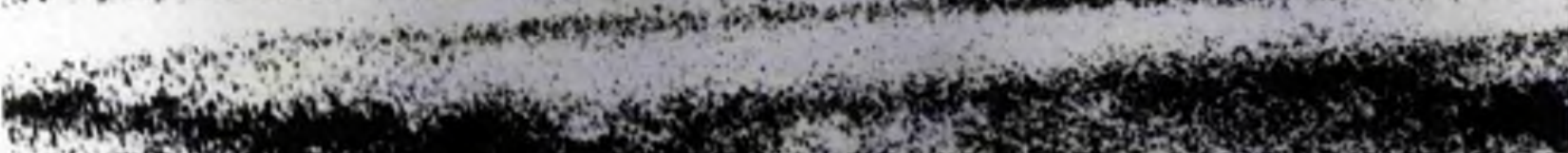

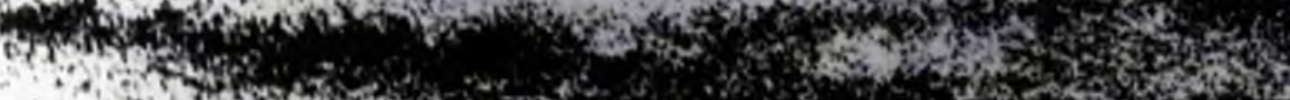

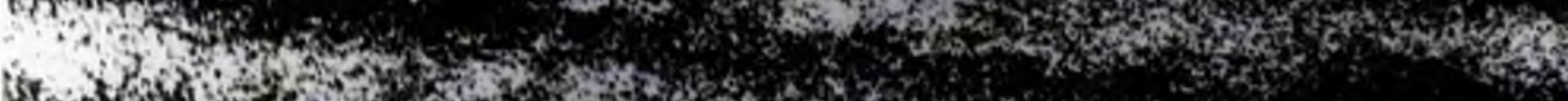

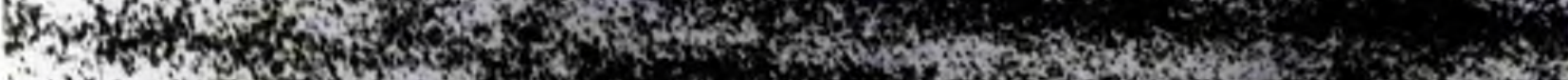

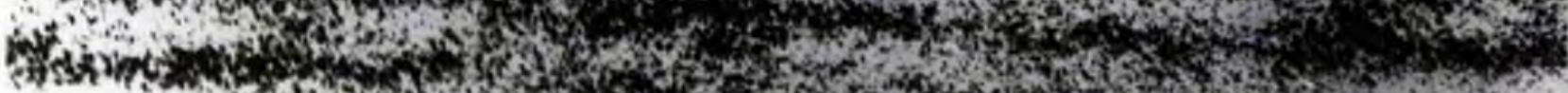

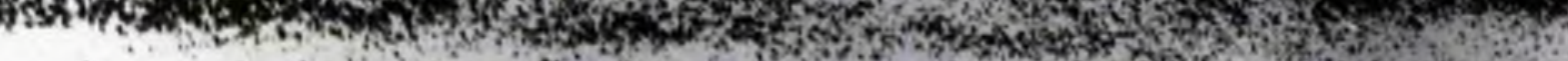

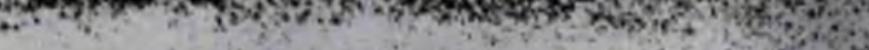

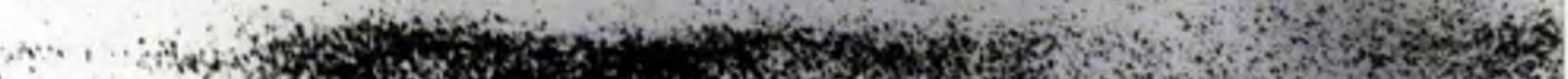

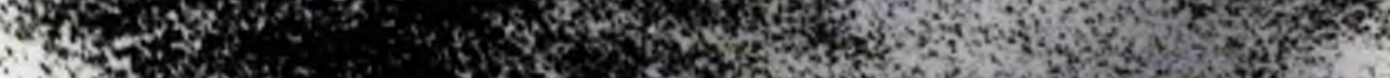

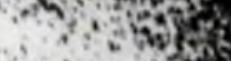

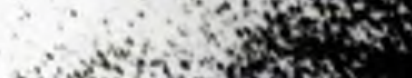

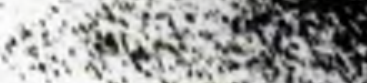

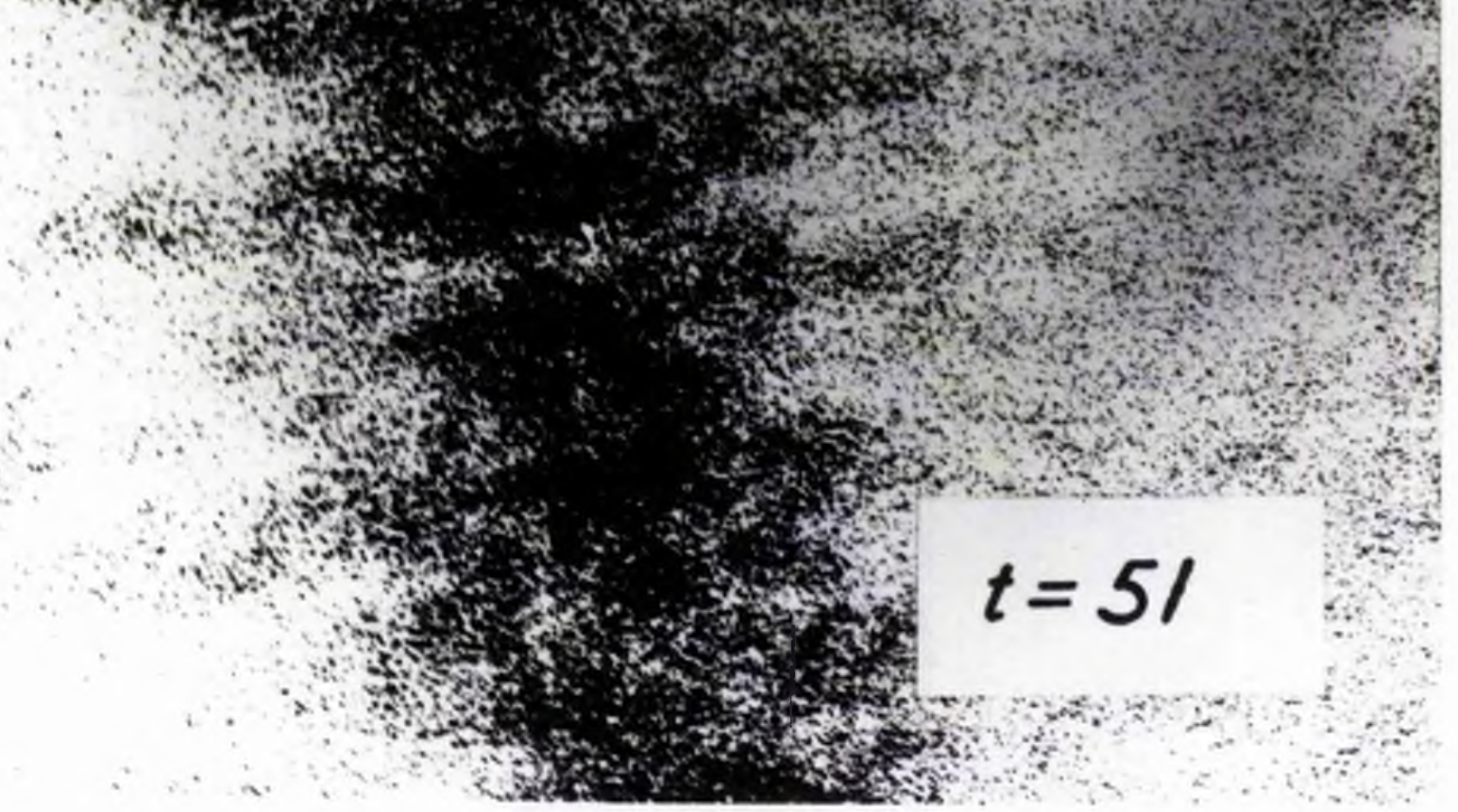

FIG. 17D 
In Fig. 18 is shown a photograph of turbulence in the Iiquid film, taken about 65 seconds after admission of $\mathrm{CO}_{2}$ to the cell (containing $0.0166 \mathrm{~N} \mathrm{NAA}$ )。 In Figiag, eddy diffusion and turbulence in the bulk of the Iiquid is clearly visible - this exposure being taken about 80 secs after the admission of $\mathrm{CO}_{2}$.

It must be emphasised that in Figs. 18. and 19 the turbulence is due almost entirely to a lack of control over the phase concentrations, - particularly the $\mathrm{CO}_{2}$. The $\mathrm{CO}_{2}$ was almost $100 \%$ pure on admission, and it is a fair surmise that the lowering of the $\mathrm{CO}_{2}$ partial pressure would eliminate this turbulence. A few test runs, using a gas burette to meter the $\mathrm{CO}_{2}$ into the cell, seem to bear this out. However, this is a reinement of the experinental technique that. will need investigating in the future. The cause of the turbuzence is a matter for conjectuxe. Possibly, the heat of reaction between $\mathrm{CO}_{2}$ and. amine may be dissipated at a somewhat greater rate than the mass transfer process. The turbulence encountered, however, would indicate a build-up of thermal energy which is onIy dissipated after a fajr length of time.

The effect of this turbulence is not of any great importance in the particular system studied. AII the 


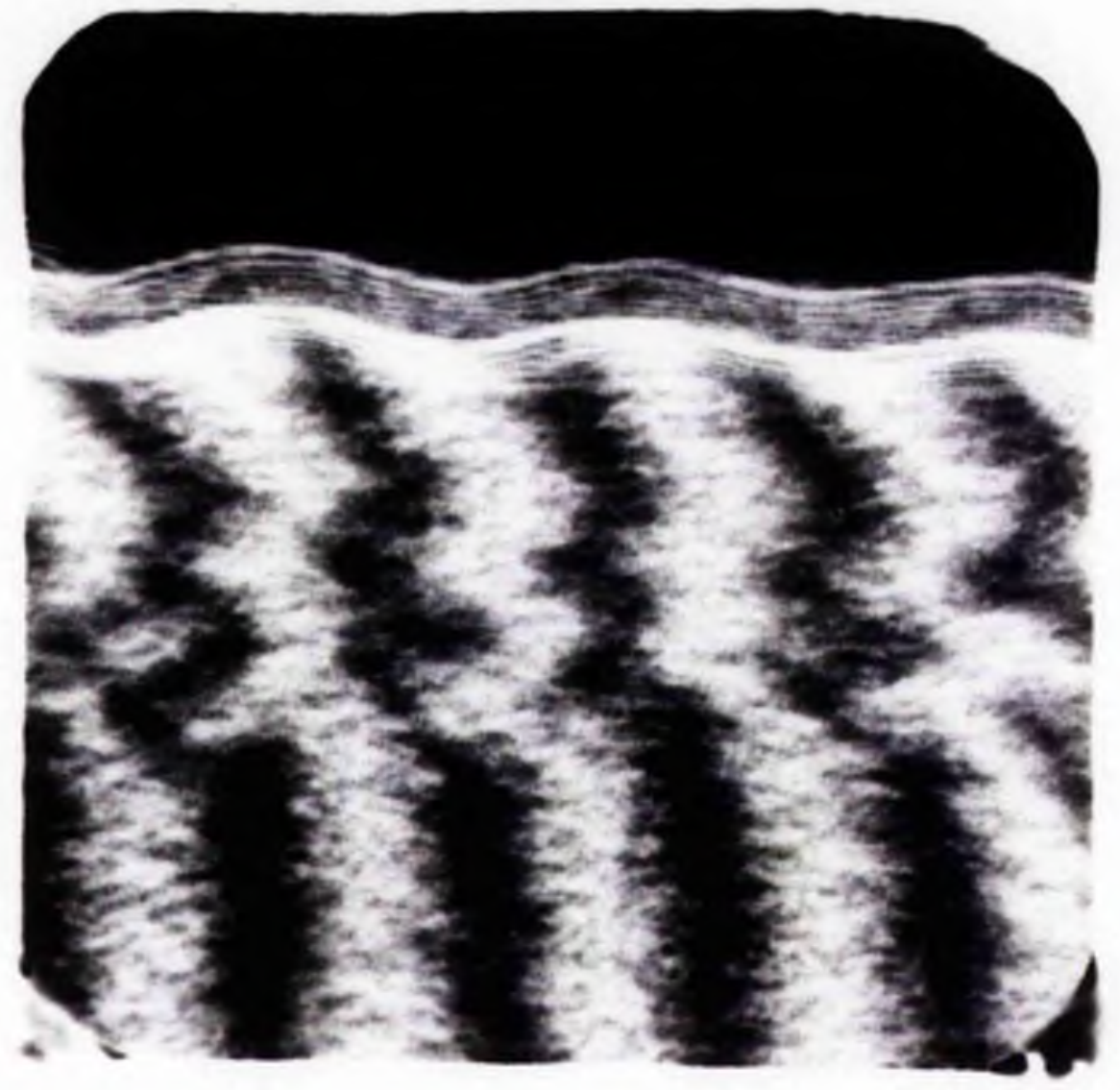

Fig.18. Turbulence in the Liquid Film.

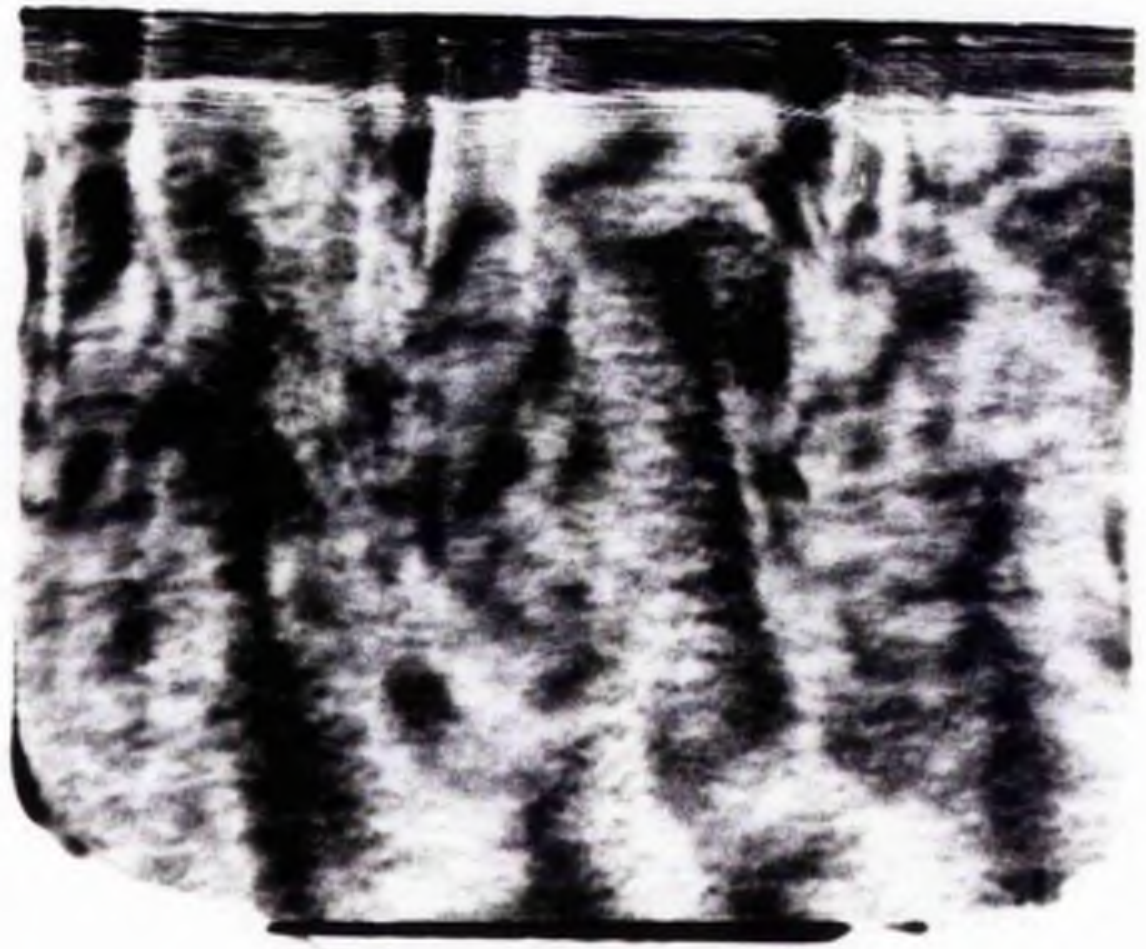

Fig.19. Turbulence in the Burk of the Liquid. 
information necessary regarding the movement of the reaction zone is registered in about 35 seconds, and the turbulence: does not follow until about 30 seconds later. Howerer, turbulence may be an important consideration with other systems.

In table 2 are recorded measurements taken in two runs for the absoxption of $\mathrm{CO}_{2}$ in $0.0766 \mathrm{~N}$ MEA. Following this table are calculations relating the experimental observations to the fIIm and penetration theories outlined earlier on.

Gaiculation of $\sqrt{\alpha}$ from graphs of $x$ against $\sqrt{\theta}$.

From the penetration theory it follows that

$$
x^{2}=2 \sqrt{\alpha} \sqrt{\theta}
$$

Thus the slope of the graph of $x^{2}$ against $\sqrt{6}$ should be $2 \sqrt{\alpha x}$. In Fig. 2I, plots of $x^{\ddagger}, \sqrt{8}$ have been made for runs $I$ and II. From which,
Run $I, \sqrt{\alpha}=0.059$
$\operatorname{Run} I I, \sqrt{\alpha}=0.070$ 
Table 2. Absorption of $\mathrm{CO}_{2}$ into $0.0166 \mathrm{~N} \mathrm{MEA}$.

\begin{tabular}{|c|c|c|c|c|c|}
\hline & $\begin{array}{c}\theta \\
\text { secs })\end{array}$ & $\sqrt{\theta}$ & $\begin{array}{l}\mathrm{x}^{2} \\
(\mathrm{~mm})\end{array}$ & $\begin{array}{c}\mathrm{x}_{\mathrm{L}}=\mathrm{x}^{\prime}+\mathrm{x}^{\prime \prime} \\
(\mathrm{mm})\end{array}$ & $\begin{array}{c}x_{i \prime \prime}^{\prime \prime} \\
(\mathrm{mmi})\end{array}$ \\
\hline Run I & $\begin{array}{r}3 \\
9 \\
15 \\
21 \\
27 \\
33 \\
39 \\
45 \\
51 \\
57 \\
63\end{array}$ & $\begin{array}{l}1.732 \\
3.00 \\
3.87 \\
4.59 \\
5.20 \\
5.74 \\
6.25 \\
6.71 \\
7.15 \\
7.55 \\
7.94\end{array}$ & $\begin{array}{l}0.140 \\
0.322 \\
0.438 \\
0.479 \\
0.578 \\
0.628 \\
0.636 \\
0.661 \\
0.628 \\
0.645 \\
0.661\end{array}$ & $\begin{array}{l}0.686 \\
0.876 \\
0.992 \\
1.100 \\
1.200 \\
1.258 \\
1.327 \\
1.327 \\
1.298 \\
1.290 \\
1.290\end{array}$ & $\begin{array}{l}0.546 \\
0.554 \\
0.554 \\
0.621 \\
0.622 \\
0.630 \\
0.691 \\
0.666 \\
0.670 \\
0.645 \\
0.629\end{array}$ \\
\hline RunII & $\begin{array}{r}5 \\
10 \\
15 \\
20 \\
25 \\
30 \\
35 \\
40 \\
45\end{array}$ & $\begin{array}{l}2.24 \\
3.16 \\
3.87 \\
4.48 \\
5.00 \\
5.48 \\
5.91 \\
6.32 \\
6.71\end{array}$ & $\begin{array}{l}0.158 \\
0.288 \\
0.379 \\
0.458 \\
0.537 \\
0.616 \\
0.663 \\
0.679 \\
0.695\end{array}$ & & \\
\hline
\end{tabular}




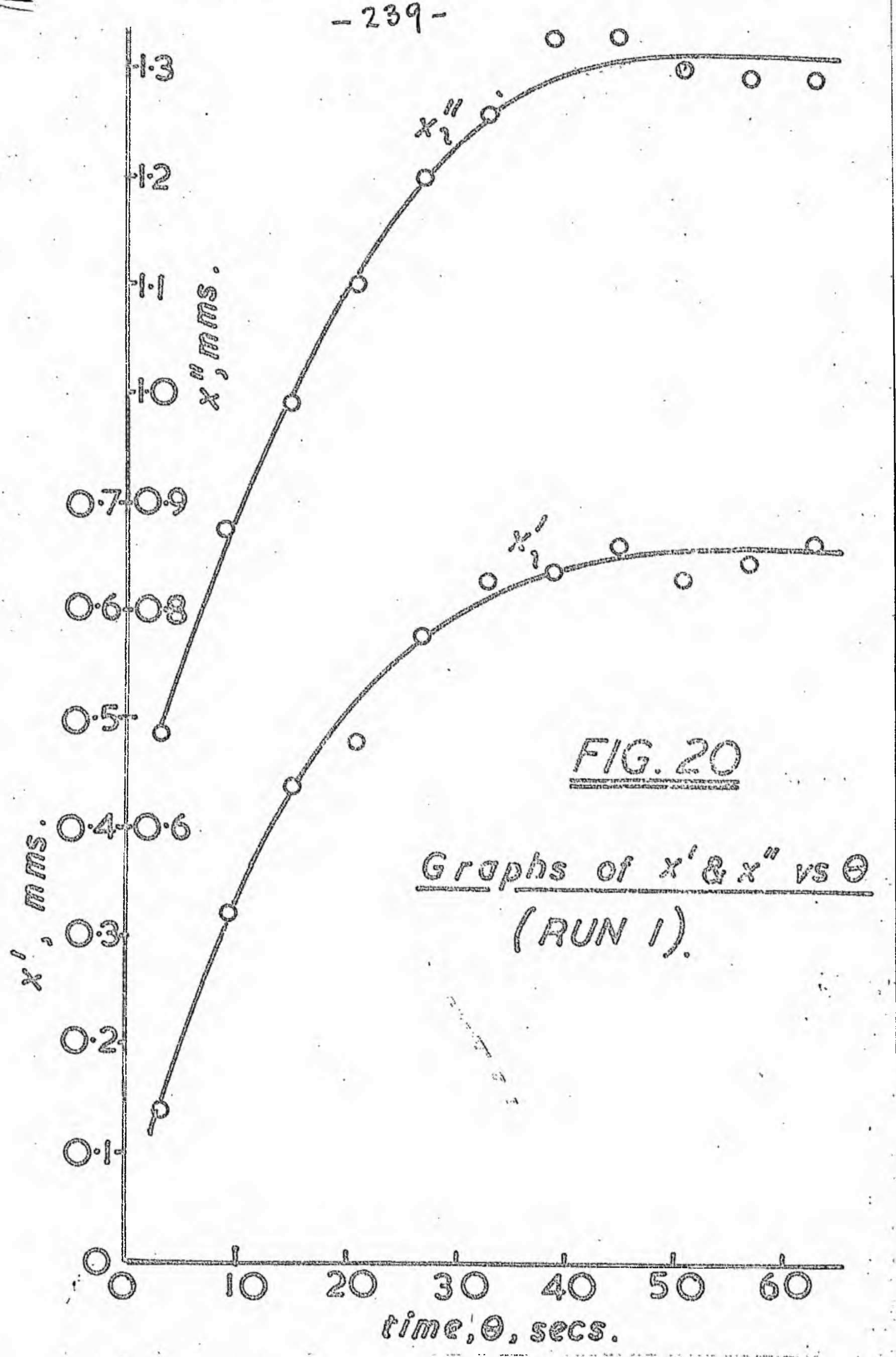


FIG. 21

Grophs of $x^{\prime}$ vs. $\sqrt{\theta}$

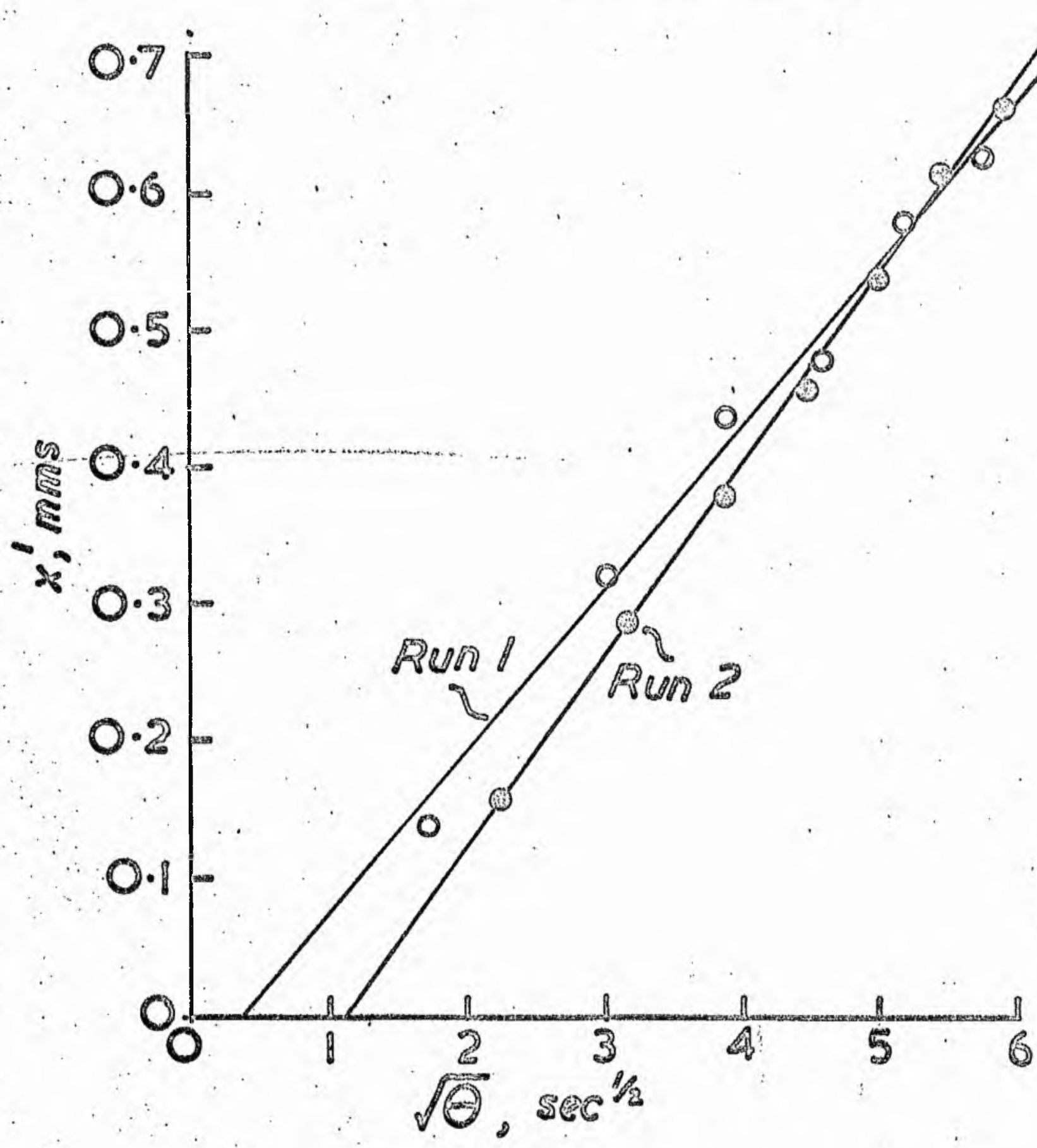


If $\left(x^{2}+x^{\prime \prime}\right)$ is plotted against $\sqrt{\theta}$, the slope gives

$$
\sqrt{O K}=0.0728(\operatorname{Run} I)
$$

In the graphs of $x^{2}$ against $\sqrt{\phi}$ it will be noticed that the line cuts the $\sqrt{6}$ maxis at a positive value of $\sqrt{9}$; this gives an indication of the exror of the timing of the photographic recording. As an estimate of the timemerror,

$$
\begin{aligned}
& \Delta \theta=0.6 \operatorname{secs}(\operatorname{Run} I) \\
& \Delta \theta=1.2 \operatorname{secs}(\operatorname{Run} I I)
\end{aligned}
$$

If the $\theta$ values are incorrect, and also the position of the "interface" for measuring $x^{3}$, then a plot of $\left(x_{a}^{p}-x_{b}^{s}\right)$ against $\left(\sqrt{\theta} a-\sqrt{\theta_{b}}\right)$ should give the true $\sqrt{\alpha}$. On this basis, by applying a loast squares fit, $\sqrt{\alpha}$ was calculited for runs $I$ and $I I$.

$$
\text { For a line } Y=A+B . X \ldots \ldots . .(46)
$$

the best slope is given by

$$
B=\frac{\mathbb{N} \sum x y-\sum x \sum x}{N \sum x^{2}-\left(\sum x\right)^{2}}
$$

where $N$ is the number of points $(\mathrm{X}, \mathrm{Y})$. In table 3, a sample calculation to calculate $\sqrt{\mathcal{O}}$ by the above method is given for run II. The first 7 points are used. 
Tabile 3o Caliculation of from Least Squares fit to

$$
\left(x_{a}^{8}-3 x_{b}\right)+\left(\sqrt{\theta_{a}}-\sqrt{\theta_{b}}\right) .
$$

\begin{tabular}{|c|c|c|c|c|}
\hline Points. & $\left(\sqrt{\theta} a-\sqrt{\theta_{b}}\right)$ & & $\left(x_{a}^{1}-x_{b}^{1}\right)$ & \\
\hline & $\mathrm{X}$ & $x^{2}$ & $Y$ & $X Y$ \\
\hline 1,2 & 0.92 & 0.8464 & 0.130 & 0.2 .1960 \\
\hline 2,3 & 0.71 & 0.5041 & 0.091 & $0.0646 I$ \\
\hline 3,4 & $0.6 I$ & 0.3721 & 0.079 & 0.04819 \\
\hline 4,5 & 0.52 & 0.2704 & 0.079 & 0.047 .08 \\
\hline 5,6 & 0.48 & 0.2304 & 0.079 & 0.03792 \\
\hline 6,7 & 0.43 & 0.7849 & 0.047 & 0.02021 \\
\hline 1,3 & 1.63 & 2.6569 & 0.221 & 0.36023 \\
\hline 1,4 & 2.24 & 5.0176 & 0.300 & 0.67200 \\
\hline 1,5 & 2.76 & $7.6 \pm 76$ & 0.359 & 0.99 .084 \\
\hline 1,6 & 3.24 & 20.4976 & 0.4 .58 & 1.48392 \\
\hline 1,7 & 3.67 & 13.4689 & 0.505 & 1.85335 \\
\hline 2,4 & 1.32 & 1.7424 & 0.170 & 0.224440 \\
\hline 2,5 & 1.84 & 3.38 .56 & 0.249 & $0.458 I 6$ \\
\hline 2,6 & $2 \cdot 32$ & 5.3824 & 0.328 & 0.76096 \\
\hline 2,7 & 2.75 & 7.5625 & 0.375 & 1.03 .125 \\
\hline 3,5 & 1.13 & 1.2769 & 0.1 .58 & 0.17854 \\
\hline 3,6 & 1.61 & 2.5921 & 0.237 & 0.38157 \\
\hline 3,7 & 2.04 & 4.1616 & 0.284 & 0.57936 \\
\hline 4,6 & 1.00 & 1.0000 & 0.158 & 0.15800 \\
\hline 4,7 & 1.43 & 2.0449 & 0.205 & 0.293 .1 .5 \\
\hline 5,7 & 0.91 & $0.828 I$ & 0.126 & 0.11466 \\
\hline$\sum$ & 33.56 & 71.6434 & 4.638 & 9.87200 \\
\hline
\end{tabular}


From this, $B=\frac{21(9.872)-(33.56)(4.638)}{21(71.6434)-(33.56)^{2}}$

$$
\begin{aligned}
& \therefore 2 \sqrt{\varepsilon=0.13658} \\
& \therefore \sqrt{\alpha}=0.0683
\end{aligned}
$$

From Run $I_{9} N=3 I$

$$
\left.\begin{array}{rl}
I_{9} N & =31 \\
\Sigma X & =36.83 \\
\Sigma X^{2} & =59.5613 \\
\sum X & =4.8 .54 \\
\sum X Y & =7.8 .5038
\end{array}\right\} \text { whence } \sqrt{\alpha}=0.0659
$$

Using the $x_{L}$ values in Run $I_{\text {, }}$

$$
\left.\begin{array}{rl}
N & =15 \\
\sum X & =27.36 \\
\sum X^{2} & =65.84112 \\
\sum X & =3.940 \\
\sum X Y & =9.09495888
\end{array}\right\} \text { whence } \sqrt{\alpha}=0.0725
$$

$\sqrt{\alpha}$ in the six calculations has been calculated to be $0.059: 0.07: 0.0728: 0.0683: 0.0659: 0.0725$.

Taking the average value,

$$
\sqrt{\alpha}=0.0681
$$

This value of $\sqrt{\alpha}$ will be used in all subsequent calculations. 
From the penetration theory, $\sigma_{A}$ is defined by

$$
\sigma_{A}=\frac{\sqrt{\alpha}}{\sqrt{D_{A}}}
$$

Using: $\sqrt{\alpha}=0.0681_{\text {s }}$ and $D_{A}=19.5 \times 10^{-4} \mathrm{~mm}^{2} / \mathrm{sec}($ since $\sqrt{\alpha}$ has been calculated on a millimetric basis)

$$
\sigma_{A}=1.54
$$

Using this value of $\sigma_{\mathrm{A}}$, it will now be shown what agreement exist,s between the experimental results obtained, and the film and penetration theories for absorption accompanied by a rapid 2nd order reaction.

1.) Taking $\begin{aligned} D_{A} & =19.5 \times 10^{-4} \mathrm{~mm}^{2} / \mathrm{sec} \\ D_{B} & =10.4 \times 10^{-4} \mathrm{~mm}^{2} / \mathrm{sec} \\ c_{A I} & =3.31 \times 10^{-5} \mathrm{~g} \cdot \mathrm{mols} / \mathrm{cm}^{3} \\ c_{B O} & =1.66 \times 10^{-5} \mathrm{~g} \cdot \mathrm{mols} / \mathrm{cm}^{3}\end{aligned}$

What $\sigma_{\mathrm{A}}$ value is needed to fit equation 33 ,

i.e. $\left(\frac{D_{B}}{D_{A}}\right)^{\frac{1}{2}}\left(\frac{c_{B O}}{c_{A i}}\right) e^{\left(\sigma_{A}^{2}\right)\left(I-\frac{1}{x}\right)} \operatorname{ext} \sigma_{A}+\operatorname{exf}\left(\frac{\sigma_{A}}{\gamma T}\right)=I$

( note: $D_{B}$ is taken as the diffusion coefficient of $O N \mathrm{NEA}$, as: measured by the author. ${ }_{A}$ is the equilibrium solubility of $\mathrm{CO}_{2}$ in water) 


$$
\begin{aligned}
& x={ }_{B}{ }_{D_{A}}=0.539 ; \sqrt{x}=0.734 ;\left(I-\frac{I}{x^{x}}\right)=-0.858
\end{aligned}
$$

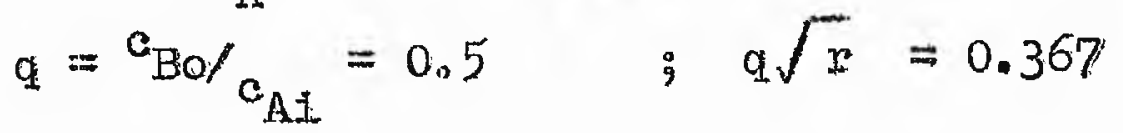

Sample calculation;-

Puite $\sigma_{A}=1$

$\therefore e^{-0.8 .58}=0.424 ; \operatorname{erf} I=0.8427 ; \operatorname{erf} I / 0.734=0.946 I$

Substituting in eqn. 33;

$$
L_{0} H_{0} S_{0}=1.077 \quad\left(R_{0} H_{0} S_{0}=I\right)
$$

$\therefore \sigma_{A}$ does not satisfy the equation

Substituting $\sigma_{A}$ values from 0.6 to 3 , it is apparent that there axe two values of $\sigma_{A}$ that satisty the equation for the above $r$ and $q$.

i.e. $\begin{array}{ll}\text { A } & \text { LHS }- \text { RHS } \\ 0.6 & -0.0856 \\ 0.7 & -0.0138 \\ 0.74 & +0.0083 \\ 0.8 & +0.0331 \\ 1.0 & +0.0770 \\ 1.2 & +0.0765 \\ 1.4 & +0.0581 \\ 1.6 & +0.0373 \\ 2.0 & +0.0116 \\ 2.2 & +0.0057 \\ 3.0 & +0.0002\end{array}$




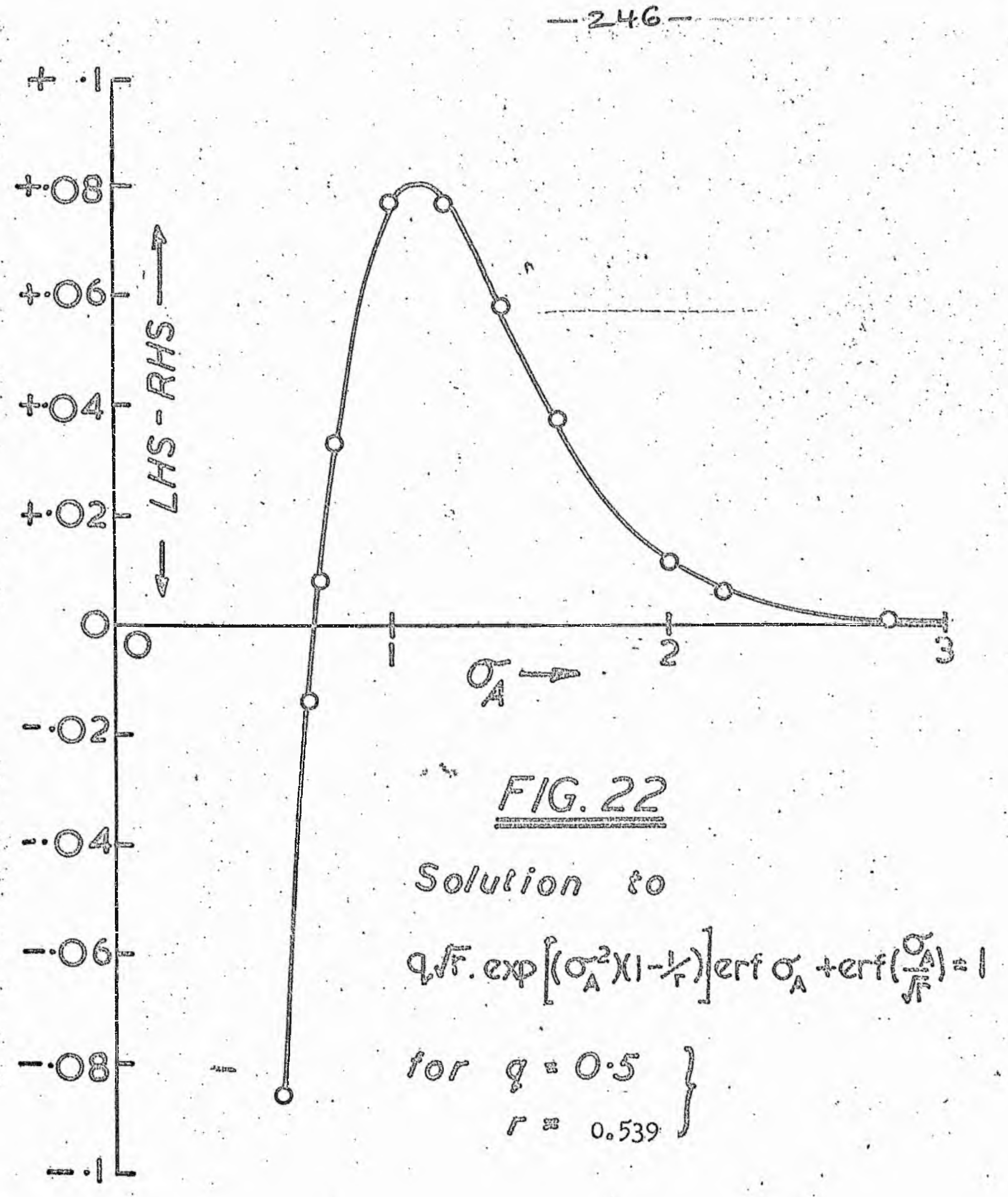


F1G.23

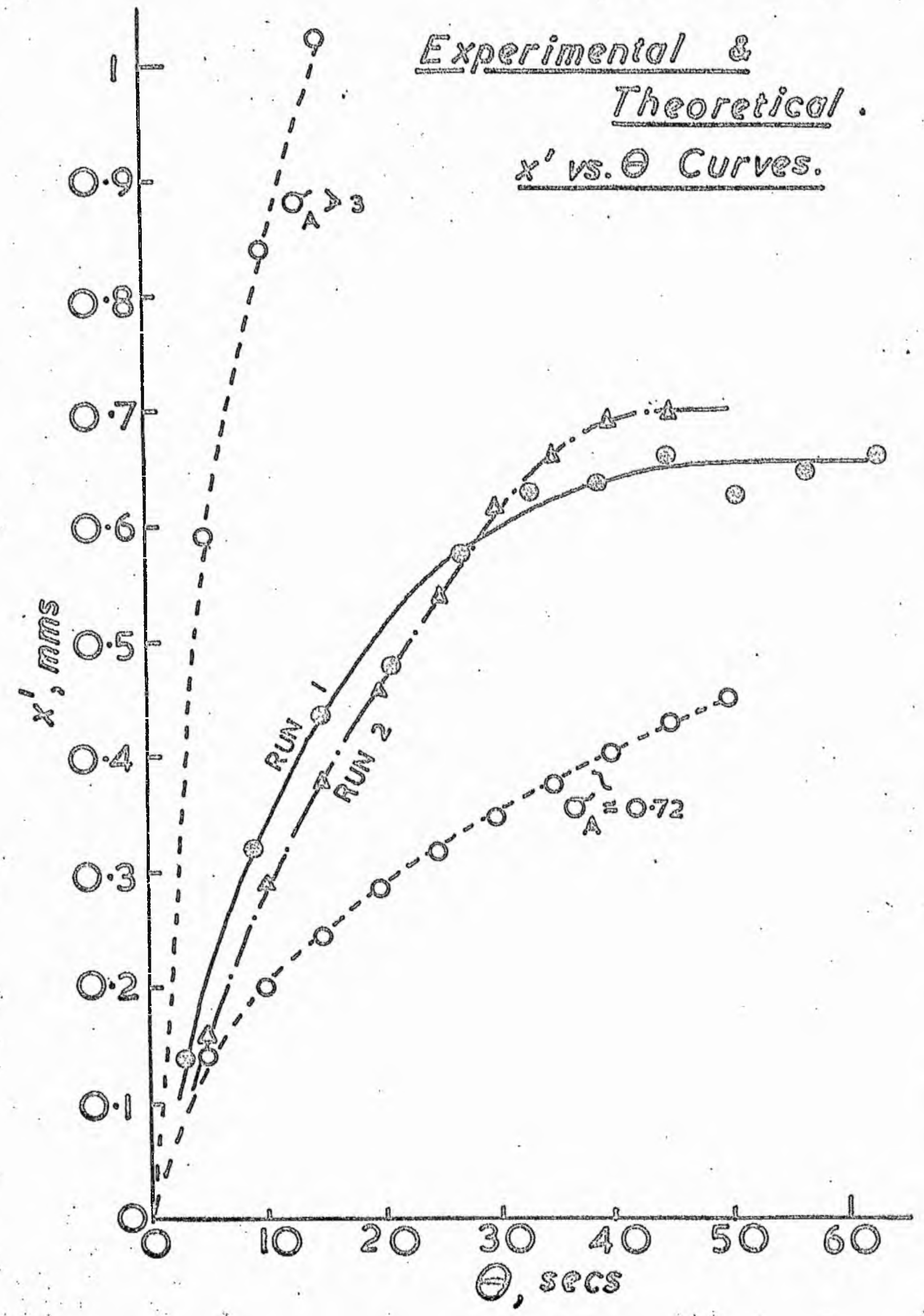




\section{$=248-$}

The solution to equation 33 , using the above $q$ and $r$, Is shown in Fig.22. From Fig.22 and the table above, the two $\sigma_{A}$ values that satisfy the equation are

$$
\begin{array}{ll}
\sigma_{A}=0.72 & (\text { real }) \\
\sigma_{A} \neq 3 & \text { (unxeal) }
\end{array}
$$

whence, $\sqrt{\alpha}=0.0318$ (reaI) and $>0.1325$ (unreal).

The theoretical movement of the reaction zone, from eqn.27, for the above $\sqrt{\alpha} \dot{\alpha}^{\prime}$ is shown in Fig. $23 ;$ and these curves are compared with the experimental points for runs I and II.

2.f From the film theory,

$$
\phi_{\mathrm{a}}=\frac{\mathrm{K}_{\mathrm{L}}}{\mathrm{K}_{\mathrm{L}}^{0}}=\frac{1}{\operatorname{erf} \sigma_{\mathrm{A}}}=1+r_{*}
$$

Taking $x=0.539, q=0.5, \quad$ then $r . q=0.2695$

$$
\text { whence } \sigma_{A}=0.883(\text { and }-\sqrt{\alpha} \bar{\alpha}=0.0389)
$$

It is thus quite obvious from calculations $I$ and 2 that the experimental values obtained for $\sqrt{\alpha}$ and $\sigma_{A}$ differ widely from the theoretical values for the system $\mathrm{CO}_{2}$ into $0.0166 \mathrm{~N} \mathrm{MEA}$, using the known values of $\mathrm{D}_{\mathrm{A}}, \mathrm{D}_{\mathrm{B}}, \mathrm{c}_{\mathrm{Ai}}$, and $c_{B O}$ to satisfy the equations. Traking $c_{A i}$ and $c_{B O}$ to 
be: correct, the problem, then, is to assess whether an alteration to the value of either $\mathrm{D}_{\mathrm{A}}$ (not very likely) or $\mathrm{D}_{\mathrm{B}}$ (more likely) will satisfy the film and penetration theories for the experimental $\sigma_{\mathrm{A}}$ value.

3.) What is $D_{A}$ to satisfy $\frac{I}{\operatorname{erf} \sigma_{A}}=I+r_{0} q$ ? Taking $\sqrt{\alpha}=0.0681, q=0.5$, and $D_{B}=20.4 \times 20^{-4} \mathrm{~mm}^{2} / \mathrm{sec}$

$$
\therefore \frac{1}{\operatorname{exP}\left(\frac{0.0681}{\sqrt{D_{A}}}\right)}=1+0.5\left(\frac{10.4 \times 10^{-4}}{D_{A}}\right)
$$

Solving in the above equation, $D_{A}$ must have to have a value of about $38 \times 10^{4-4} \mathrm{~mm}^{2} / \mathrm{sec}$.

4. What is $D_{A}$ to satisfy

$$
q \sqrt{x} \theta\left(\sigma_{A}^{2}\right)\left(I-1 / I_{2}\right) \operatorname{exf} \sigma_{A}+\operatorname{exf} \sigma_{A} / \sqrt{I}=I_{9}
$$

taking; the above $\sqrt{\alpha}, q$, and $D_{B}$ values?

$$
\begin{array}{lc}
\text { Solving in the above equation, } \\
\mathrm{D}_{\mathrm{A}}\left(\mathrm{x} I \mathrm{O}^{4}\right) & \text { LHS RHS } \\
20 & +0.026 \\
40 & +0.0052 \\
45 & +0.0037 \\
50 & +0.0026 \\
70 & +0.0003
\end{array}
$$


Thus, to explain the $\sqrt{\alpha}$ vailue obtained experimentally, assuming: $D_{B}, c_{A 1}$, and $c_{B o}$ to be correct, $D_{A}$ would have to be about $38 \times 10^{-4} \mathrm{~mm}^{2} / \mathrm{sec}$ (film theory, calc. 3 ) or greatex than $70 \times 10^{-4} \mathrm{~mm}^{2} / \mathrm{sec}$ (penetration theory , calc. 4 ). Since the only means of increasing $\mathrm{D}_{\mathrm{A}}$ by this extent would be a temperature rise of a good 20 to $30^{\circ} \mathrm{C}$, It would appear that $D_{B}$ needs re-examination ( assuming this time that $D_{A}$ is correct).

5./ What is $D_{B}$ to satisfy $\frac{1}{e r^{2} \sigma_{A}}=I+x \cdot q$ ?

$$
\begin{aligned}
& \text { taking } \sigma_{A}=1.54, q=0.5 \text {. } \\
& y=\frac{\left(\frac{1}{\operatorname{exf} 1.54}-1\right)}{0.5}=0.06
\end{aligned}
$$

whence $D_{B}=1.73 \times 10^{-4} \mathrm{~mm}^{2} /$ sec.

6./ What is $D_{B}$ to satisfy eqn 33 , as in calculation 48 Solving, using the $\sigma_{A}$ and $q$ values as in caliculation 5 ,

$$
\begin{array}{cl}
\mathrm{D}_{\mathrm{B}}\left(\mathrm{x} \mathrm{I0} 0^{4}\right) & \text { IHS }-\mathrm{RHS} \\
10.4 & 6 \times 10^{-2} \\
5 & 1 \times 10^{-4} \\
4 & 9 \times 10^{-6} \\
2 & 4 \times 10^{-11}
\end{array}
$$


From calculations 5 and 6 , it can be seen that by lowering $D_{B}$ to a value of around $2 \times 10^{-4} \mathrm{~mm}^{2} / \mathrm{sec}$, the experimental. $\sqrt{\alpha}$ value is verified to an extent.

It would appear then that the reaction zone observed in the runs described above gives rise to an axtifin icially low value of $\sqrt{\alpha}$, the likeliest explanation of which is a lowering of $D_{B}$.

There is a fals amount of justification in making this statement, - cortainly in view of the long contact time employed, and this will be discussed in the following section.

One point remains in clarifying the above calculiattions - Presuming the interface had been wrongly identified, and simultaneously the timing of the photographic recording. was Incorrect by about 2 seconds ( large error) ), would this have much effect on $\sqrt{\alpha}$ ? As a test of this particular case, a theoretical curve was drawn, and was then displaced by appreciable increments in $x^{3}$ and $\theta$. On plotting $x^{\prime}$ against: $\sqrt{\theta}$ the effect is to shift the curve sufficiently to cut the $\theta$ - axis at a negative value when $x^{\prime}=0$. By compaxing this displaced curve and the experimental curtes, It is quite evident that the interface has been fairly accurately identified. Also $\sqrt{\alpha}$ is not altered much by displacing the curve. 
$-252=$ 


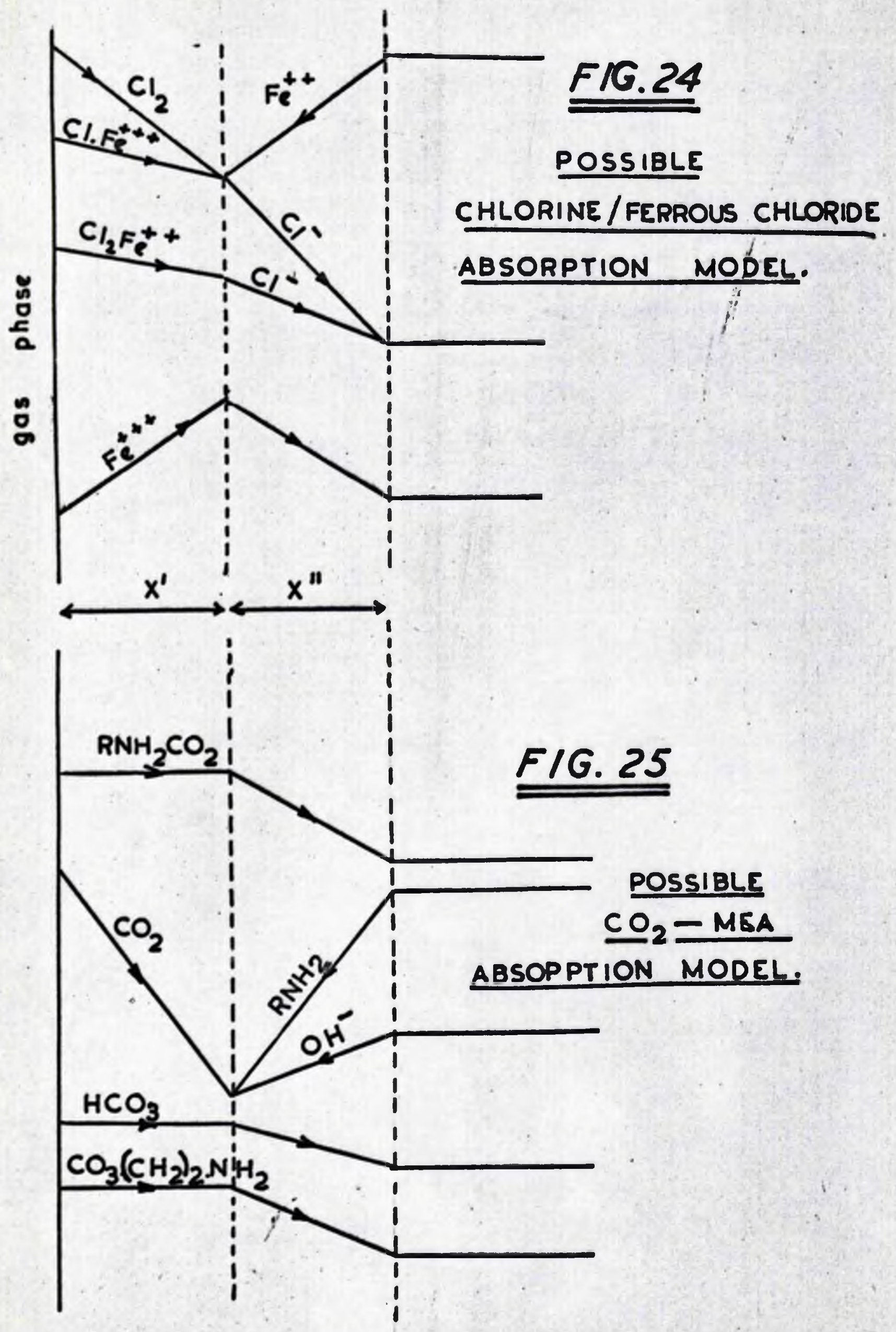


The Reduction of $\mathrm{D}_{\mathrm{B}}$ in the Absorption of $\mathrm{CO}_{2}$ by MEA.

In an experimental investigation of the absorption of chloxine by ferrous chtoride in hydrochloric acia, Gilliland et al ( 88 ) concluded that the effective difrusm Ivity of the ferrous ion was only $59 \%$ that of the salt diffusivity of ferrous chloride. The overall reaction may be expressed as

$$
\mathrm{CI}_{2}+\mathrm{Fe}^{++} \rightarrow 2 \mathrm{CI}+2 \mathrm{Fe}^{++++}
$$

However, this reaction may be broken down into three stages,
1. $\mathrm{CI}_{2}+\mathrm{Fe}^{++} \rightarrow \mathrm{Cl}_{2} \cdot \mathrm{Fe}^{++}$
..........(49)
2) $\mathrm{CI}_{2} \cdot \mathrm{Fe}^{+++} \rightarrow \mathrm{CI}^{-}+\mathrm{CI}_{4} \mathrm{Ee}^{+++}$
3) $\mathrm{CL}_{\mathrm{Fe}} \mathrm{Fe}^{++t}+\mathrm{Fe}^{++} \rightarrow \mathrm{Cl}^{-}+2 \mathrm{Fe}^{+++t}$

Stage 2 is slow relative to stages $I$ and 2 . Thus, a fairly complex ionic reaction model is: formulated, and it becomes evident that $D_{B}$ is no longex merely the diffusion coefficient for the ferrous salt. diffusing towards the reaction zone. Instead, $D_{B}$ must takse into account the complex ionic counterdiffusion occuring as the reaction proceed.s.

Using the Hatta film theory model, the absorption of chlorine by ferrous chloride may thus probabaly be represented by Fig.24. 
For the absoxption of $\mathrm{CO}_{2}$ by $\mathrm{MEA}$, the rate $\mathrm{Ls}$ governed by three main simultaneous reactions.

$$
\begin{aligned}
& \text { i.e. I) } \mathrm{R} \cdot \mathrm{NH}_{2}+\mathrm{CO}_{2} \rightarrow \mathrm{R}_{0} \mathrm{NH}_{2} \cdot \mathrm{CO}_{2} \\
& \text { (carbamate) } \\
& \text { 2) } \mathrm{OH}^{-}+\mathrm{CO}_{2} \rightarrow \mathrm{H}_{3} \mathrm{CO}_{3} \\
& \text { 3) R. } \mathrm{NH}_{2}+\mathrm{OH}^{\mathrm{N}}+\mathrm{CO}_{2} \rightarrow \mathrm{CO}_{2} \cdot \mathrm{O} \cdot \mathrm{CH}_{2} \mathrm{CH}_{2} \mathrm{NHH}_{2}+\mathrm{H}_{2} \mathrm{O}
\end{aligned}
$$

A possible representation of the absorption, based on the Hattita model, would be as in Fig.25.

As with Fig. 24, Fig. 25 is only a representationali diagram. However, it can be seen that the diffusion of the amine is very probabaly countered by the diffusion of the reaction products. With the absorption of chjoxine by ferrous chloride, the controlling reactions are strongly lonic, and the maintenance of electrical neutrality will govern the diffusional processes. With the absorption of $\mathrm{CO}_{2}$ by: MEA, the reactions are not strongly ionic, but it is very: likely that significant forces of interaction are present, as with the plain diffusion of amine in water. With the experimental technique employed in. observing the diffusion of $\mathrm{CO}_{2}$ in MEA, comparatively large contact times are employed. There is a strong possibility; therefore, that the reaction products formed in this time 
will interfere with the diffusion of the amine to a fax greater extent than that observable ing say, the laminar jet with a contact time of the order of 0.005 seconds. As a possible indication of this, it can be seen in Fig. 23 that the position of the experimentally observed reaction zone aftex 5 seconds is not very different from that of the theoretical reaction zone position for $D_{B}=3.0 .4 \times 10^{\mathrm{m}}$ $\mathrm{mm}^{2} /$ sec. Only at the Ionger contact times does the experimental curve differ widely from the curve for $D_{B}=1.0 .4 \times 10^{-4}$. It would thus appear that the counterdiffusion of the amine and the reaction products ( formed in the reaction between $\mathrm{CO}_{2}$ and $\mathrm{MEA}$ ) will significantly affect the value of $D_{B}$ at. long contact times.

A further insight into the influence on $D_{B}$ of: ionic counterdiffusion is given by sherwood and Wei ( 97 ). However, in the systems they examined, - HCI in NaOH, and acetic acid in $\mathrm{NaOH}$, the value of $\mathrm{D}_{\mathrm{B}}$ is increased. For the absorption of $\mathrm{HCl}$ in $\mathrm{NaOH}$, a rigoxous treatment of the ionic counterdiffuston occurring lieads to a prediction of a. doubling of the mass transfer rate over that predicted by the simple film theory.

Further experimental observations are needed to validate the decrease in $\mathrm{D}_{\mathrm{B}}$ for the absorption of $\mathrm{CO}_{2}$ in 
amine. However, 1t can be safely assumed at this stage that $D_{B}$ is decreased due to the counterdiffusion of the amine and reaction products at the long contact times employed. 
IV. SUMMARY

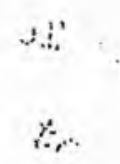

To summarise the study made of the application of the wavefront shearing interferometer to gas-liquid diffusion, the following observations are made;-

1) Having proved the wavefront shearing: interferometer as being a highly accurate and sensitive instrument for the measurement of mutual diffusion rates. in binary liquid systems, a new diffusional cell was designed and constructed for gas-liquid contacting. An experimental technique was evolved for admitting the gas to the cell, for contacting with the liquid, with the minimum change in the physical conditions of the system. Since only a very slight thermal imbalance is detectable by the interferometer, it is essential that the physical conditions in the cell remain as stiable as possible.

2) The first gas-liquid system investigated using the wavefront shearing interferometer was the diffusion of $\mathrm{CO}_{2}$ into water. It was expected from the outset that there would not be a great enough refractive index difference present to make any accurate measurements possible. A preliminary check on the refractive index of $\mathrm{CO}_{2}$-saturated 
water indicated that the detection of the absorption of $\mathrm{CO}_{2}$ lay water was at the limit of the sensitivity of the interferometer. The experimental results confirmed this expectation. Thus, the wavefront shearing interferometer can only have a limited application for diffusion measures. mentis with a gas of sparing solubility in a solution. In the light of this, the results of previous workers who who used less sensitive interferometers to study mass transfer in the $\mathrm{CO}_{2}$-watior system, may be said to need critical re-examination. There is no reason to doubt, however, that the wavefront shearing interferometer could not be employed with success with a more soluble gas.

3) The second system to be investigated was the absorption of $\mathrm{CO}_{2}$ by dilute monoethanolamine solution, - the diffusion of $\mathrm{CO}_{2}$ in this case being accompanied by a fast 2nd order reaction. After a number of trial and exror runs, in which a successful experimental technique was finally evolved, a major success was accomplished. This consisted of the showing experimentally, for the first time, of the existence of a distinct reaction zone in the liquid film. The movement of this reaction zone was recorded photographicaling. 
4) Measurements taken of the reaction zone movement observed in the absorption of $\mathrm{CO}_{2}$ by dilute MEA, Indicated that the value of $\mathrm{D}_{\mathrm{B}^{9}}$ - the diffusion coefficient for the amine, was only about $20 \%$ of its true value. This has been explained as being caused by countexdiffusion of amine and the reaction products at the large contact time employed.

5) In view of the successful illustration of diffusion with reaction in the liquid film, there is every possibility that a completely new field of interferometric experimental investigation in mass transfer is opened up. A good deal more experience is called for, and also a remappraisal of the experimental technique involved.

\section{Suggestions Tor Further Work.}

In the immediate future, improvement in the experimental technique for operating the gasmliquid cell is of first priority. These improvements may be itemised a.s below; -

1) Control of the gas concentrations on admission, such that the heat of reaction is minimised. The experimentali 
Errangement shown in Fig. IOA is a first step towards this.

2) Increase the intensity of the illumination, so that the photographic registration (at: short exposure thimes) can be made of the initial diffusional process (say, within the first 5 seconds ). Arrangements are being made at the moment to put this matter in hand.

3) Improve the image measurement. The use of an instrument as accurate as the recording microdensitometer is suggested for measuring the movement of the reaction zone.

4) Although not as vital as the above suggestions, a remappraisal of the gas-liquid cell design is called for. The present dimensions, $6.3 \mathrm{~cm}$ path length $\times 5 \mathrm{~cm}$ wide $\times 4 \mathrm{~cm}$ deep could possibly be changed to advantenge. A cell $2 \mathrm{~cm}$ wide would be quite sufficient, and would make the engineering of the knife-edge easier. Also, an alteration of the path length might increase the sensitivity $y_{9}$ though providing no optical aberrations are introduced.

5) A further series of runs on the absorption of $\mathrm{CO}_{2}$ in dilute $\mathrm{MEA}$ is desirable. The use of $\mathrm{d}$ - and triethanolamines as solvents could also be profitably studied. Other systems, with slower reaction rates, are also of interest. 
$-2611-$

\section{APPENDIX I}

Solution to Fick's and Law. 


\section{APPENDIX I}

\section{Solution to Fick's Second Law.}

It can be shown by dimensional analysis that a solution of

$$
\frac{\partial c}{\partial t}=D \cdot \frac{\partial^{2} c}{\partial x^{2}}
$$

Is: obtained as

$$
c=\frac{A}{\sqrt{t}} \exp \left(-\frac{x^{2}}{4 D t}\right)
$$

The derivation is lengthy, however, so that in this work it will be shown that equation $(B)$ is a solution of $(A)$.

Differentiating: (B) with respect to th

$$
\begin{aligned}
\frac{\partial c}{\partial t} & =A\left[e^{-x^{2}} 4 D t\left(-\frac{1}{2}\right) t^{-3 / 2}+\frac{1}{\sqrt{t}}\left(\frac{x^{2}}{4 D t^{2}}\right) e^{-x^{2}} 4 D t\right] \\
& =A_{0} e^{-x^{2} / 4 D t} \cdot t^{-3 / 2}\left[\left(-\frac{x}{2}\right)+t \cdot\left(\frac{x^{2}}{4 D t^{2}}\right)\right] \\
& =\frac{A}{2} \cdot e^{-x^{2} / 4 D t} \cdot t^{-3 / 2}\left[\frac{x^{2}}{2 D t}-1\right] \ldots \ldots
\end{aligned}
$$

Differentiating (Bi) with respect to $\mathrm{x}$, 
$-263-$

$$
\begin{aligned}
\therefore \frac{\partial^{2} c}{\partial x^{2}} & =\frac{A}{\sqrt{t}}\left[\left(\frac{2 x}{4 D t}\right)^{2} \cdot e^{-x^{2} / 4 D t}-\frac{2}{4 D D} \cdot e^{-x^{2} / 4 D t}\right] \\
& =\frac{A}{t} \cdot e^{-x^{2} / 4 D t} \cdot \frac{1}{2 D t}\left[\frac{x^{2}}{2 D t}-\frac{1}{2}\right] \ldots
\end{aligned}
$$

Multiply (E) by,$D$ (diffusivity),

$$
\begin{aligned}
& D \cdot \frac{\partial^{2} c}{\partial x^{2}}=\frac{A}{2} \cdot e^{-x^{2} / 4 D t} \cdot t^{-3 / 2}\left[\frac{x^{2}}{2 D t}-1\right] \\
& \therefore \quad D \cdot \frac{\partial^{2} c}{\partial x^{2}}=\frac{\partial c}{\partial t}
\end{aligned}
$$

And therefore $C=\frac{A}{\sqrt{t}} \cdot e^{-x^{2} / 4 D t}$ is a solution to this equation. 


$$
-264=
$$

APPENDIX II

The Taylor Series Expansion of

$$
\left(\frac{\Delta \mathrm{z}}{\Delta \mathrm{x}}\right)_{\Delta \mathrm{x}=\mathrm{b}}
$$




\section{The Taylor Expansion ( 98 ).}

Taylor's theorem determines the law for the expansion of a function of the sum of two variables into series of ascending powers of one of the variables.

Assume $\quad u_{i}=f(x+y)=A+B y+C y^{2}+D y^{3} \ldots$

Let $A^{4}, B^{8} C^{*} \ldots$ be the respective values of $A_{3} B, C, \ldots i n(I)$ when $x=a \quad(a=a n$ arbitrary value).

$\because u^{\prime}=f^{\prime}(a+y)=A^{\prime}+B^{\prime} y+C^{\prime} \cdot y^{2}+D^{\prime} y^{3} \ldots$

Putting; $z=a+y \cdot \because y=z-a$,

$$
\mathfrak{u}^{2}=f(z)=A^{\prime}+B^{\prime}(z-a)+C^{\prime}(z-a)^{2}+D^{\prime}(z-a)^{3} \ldots
$$

Differentiating with respect to $z_{2}$

$$
\begin{aligned}
& \frac{\partial u^{\prime}}{\partial z}=\mathrm{I}^{\prime}(z)=B^{i}+2 C^{\prime}(z-a)+3 D^{\prime}(z-a)^{2} \\
& \frac{\partial^{2} u}{\partial z^{2}}=f^{2} \cdot(z)=2 C t+2 \cdot 3 D \cdot(z-a)+3 \cdot 4 t^{\prime}(z m a)^{2} \ldots . \\
& \frac{\partial^{3} u^{2}}{\partial z^{3}}=I^{2}+1(z)=2 \cdot 3 \cdot D^{1}+2 \cdot 3 \cdot 4 E^{3}(z-a)
\end{aligned}
$$

Putting $z=a$, and $\therefore y=0$, 
$f(a)=A^{2} ; f^{\prime}(a)=\mathbb{B}^{3} ;(a)=20^{8} ; f^{411}(a)=2.301 ;$

$\therefore C=\frac{1}{2} f^{\prime}(a)$ and $D=I / 2.3^{11}(a) \quad \ldots \ldots$ (4)

Substituting (4) into (2),

$u^{t}=f(a+y)=f(a)+\frac{f(a)}{1 !} \cdot y+\frac{f \cdot 1(a)}{2 !} \cdot y^{2}+\frac{f+1 \cdot(a)}{3 !} \cdot y^{3} \cdots$

Buti if $a$ is any value of $x$,

$u=f(x+y)=f(x)+\frac{f^{\prime}(x)}{1 !} \cdot y+\frac{f^{3}+(x)}{2 !} \cdot y^{2}+\frac{f^{\prime \prime 1}(x)}{3 !} \cdot y^{3} \ldots \ldots$

And similariy,

$u:=f(x-y)=f(x)-\frac{f^{\prime}(x)}{1 !} \cdot y+\frac{f^{4} \cdot(x)}{2 !} \cdot y^{2}-\frac{f^{311}(x)}{3 !} \cdot y^{3} \cdots \cdots$

Equations (6) and (7) are the Taylor Series expanstons of $f(x+y)$ and $f(x-y)$ respectively.

$$
\frac{\text { Expansion of }\left(\frac{\Delta_{z}}{\Delta x}\right)}{\left(\frac{\Delta_{z}}{\Delta x}\right)_{\Delta x=b}=\frac{f(x+5 / 2)-f(x-b / 2)}{b}}
$$

expanding,

$$
f^{\prime}(x+b / 2)=f(x)+\frac{f^{\prime}(x)}{1 !} \cdot \frac{b}{2}+\frac{f^{1 !}(x)}{2 !} \cdot \frac{b^{2}}{4}+\frac{f^{11}+(x)}{3 !} \cdot \frac{b^{3}}{8} \cdots
$$


$-267-$

$$
\begin{aligned}
& f(x-b / 2)=f(x)-\frac{f^{\prime}(x)}{1 !} \cdot \frac{b}{2}+\frac{f^{8}+(x)}{2 !} \cdot \frac{b^{2}}{4}-\frac{f^{11}+(x)}{3 !} \cdot \frac{b^{3}}{8} \cdots \\
& \therefore \frac{f(x+b / 2)-f(x-b / 2)}{b}=\frac{1}{b}\left[f^{\prime}(x) \cdot b+\frac{2 f^{\prime 11}(x) \cdot b^{3}}{3 !}\right. \\
& \left.+\frac{2 f^{V}(x)}{5 !} \cdot \frac{b^{5}}{64} \cdots \cdots\right]
\end{aligned}
$$

$$
\begin{aligned}
& \text { Now } f(x)=\frac{\partial n}{\partial x}=A \cdot e^{-x^{2} / B} \\
& \text { where } A=\frac{\Delta n}{\sqrt[2]{\pi D t}} \text { and } B=4 D t \text {. } \\
& \therefore f^{\prime \prime}(x)=-\left(\frac{2 x}{B}\right) A \cdot e^{-x^{2} / B} \\
& \therefore \operatorname{fit}(x)=-\left(\frac{2 x}{B}\right) \cdot f^{t}(x) \\
& f^{\prime \prime \prime}(x)=\left[\frac{2}{B} \cdot \mathbf{I}^{\prime}(x)\right]+\left[\left(-\frac{2 x}{B}\right)\left(-\frac{2 x}{B}\right) \cdot f^{\prime}(x)\right] \\
& =\frac{2}{B^{2}} \cdot f(x) \quad\left[2 x^{2}-B\right] \\
& f^{I V}(x)=\left[\left(\frac{2}{B^{2}} \cdot P^{11}(x)\right)\left(2 x^{2}-B\right)\right]+\left[\frac{2}{B^{2}} \cdot P^{\prime}(x) \cdot 4 x\right] \\
& \because f^{I I}(x)=\frac{2}{B^{3}} \cdot f^{\prime}(x) \cdot\left[6 x B-4 x^{3}\right]
\end{aligned}
$$




$$
\begin{aligned}
& f^{V}(x)=\left[\frac{2}{B^{3}}\left(-\frac{2 x}{B}\right) f^{\prime}(x) \cdot\left(6 x B-4 x^{3}\right)\right]+\left[\frac{2}{B^{3}} \cdot f^{\prime}(x)\left(6 B-12 x^{2}\right)\right] \\
& \therefore f^{V}(x)=\frac{4}{B^{4}} \cdot f^{\prime}(x)\left[4 x^{4}-12 x^{2} B+3 B^{2}\right] \quad \ldots \ldots(16)
\end{aligned}
$$

Substituting: 12,14 , and 16 into. 11 ,

$$
\begin{aligned}
\left(\frac{\Delta z}{\Delta x}\right)_{\Delta x=b}=\left\{f^{\prime}(x)+\right. & f^{\prime}(x) \cdot \frac{2}{B^{2}} \cdot \frac{\left(2 x^{2}-B\right)}{3 !}\left(\frac{b}{2}\right)^{2} \\
& \left.+f^{\prime}(x) \cdot \frac{4}{B^{4}} \cdot \frac{\left(4 x^{4}-12 x^{2} B+3 B^{2}\right) \cdot\left(\frac{b}{4}\right)^{4}}{5 !} \cdots\right\} \\
= & f^{\prime 2}(x)\left[1+\frac{2}{B^{2}} \cdot \frac{\left(2 x^{2}-B\right)}{3 !}\left(\frac{b}{2}\right)^{2}\right. \\
& +\frac{4}{B^{4}} \cdot \frac{\left.\left(4 x^{4}-12 x^{2} B+3 B^{2}\right) \cdot\left(\frac{b}{4}\right)^{4} \cdots\right]}{5 !}
\end{aligned}
$$

Substituting $B=4 D t$ and putting $f^{\prime}(x)=\frac{\partial n}{\partial x}$,

$$
\begin{aligned}
&\left(\frac{\Delta z}{\Delta x}\right)_{\Delta x=b}= \frac{\partial n}{\partial x}\left\{I+\frac{2}{2^{2} \cdot 3 !}\left(\frac{b^{2}}{2 D t}\right)\left(\frac{x^{2}-2 D t}{2 D t}\right)\right. \\
&+\frac{1}{2^{4} \cdot 5 !}\left(\frac{b^{2}}{2 D t}\right)^{2} \\
&\left.\left.\frac{\left(x^{4}-12 x^{2} D t+12 D^{2} t^{2}\right.}{4 D^{2} t^{2}}\right) \ldots\right\}
\end{aligned}
$$




$$
-269-
$$

\section{APPENDIX III}

Computer Programme (Sirius Autocode) for the Caliculation of D (Least squares curve ritting). 
$N$

COMPUTATION OF DRFFUSION COEFFIGIENT.

BEST D AND T(INFLO) FOUND BY THE BETHOO OF LEAST SQUARES. APPLYING EXPERIMENTAL DATA.

TIME INCREMENS ADDED BEFORE CALCULATING D.

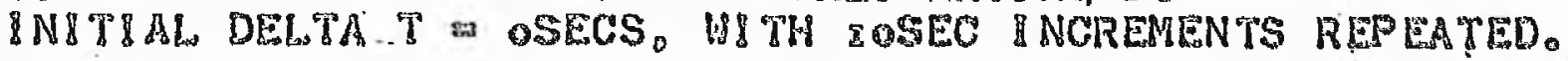

IDEAL CURVE 90 FIT BEST D AND T ALSO CALCULATEDO ALSO YARTANCE OF ERPTL FROM THORETHCAL PONNTS.

$v=\pi 507$.

II 5

ปण

ALeito

A.

$56 \times 5 \times 5$

$56 \& 6=26$

$5687^{\mathrm{an}} \mathrm{g} 7$

TS

ปขณ

VOO $x=$ TAPE:

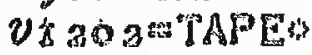

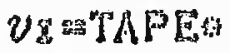

V

nis $a n 0$.

V8000 $=0$

$11 \times 0200$

"TER "T"

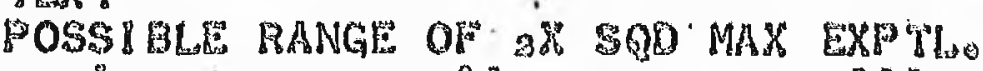
8 DI 18

$v_{908}=v_{90} / x a 95$

$v_{909}=008 \times 2008$

vososogos/30ys

$v_{9} \times x^{3} v_{9} y_{0} \times v_{9} \times 0$

$v_{906} 0003 / 3$ ass

v9oyw2906\% 2906

$v_{9} 8=004 / 2095$

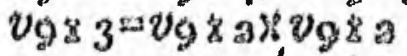

$v_{9} \times 4=v_{9}$ os/ 590

PRNA PRINTitoogo

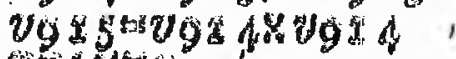

pat Nito

PM

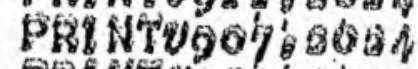

PRINTUG S

PRINTHES 
8) $v(308+n s 0)=v(305+580) / 8 \circ 925$

$v(803+1850)=v(x 084850) \times 2)(508+4350)$

$n \times 0=n \times 0+8$

$\Rightarrow 4.750$ ons

is $30=0$

: 20$)$ PRINTV( $x+430) \cdot 3080$

PRINTV( $305 \div 450), 4034$

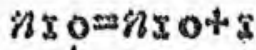

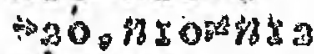

5) $18 \times 0=0$

TEXT.

DELISA T

D

I

$\therefore X$ SQD

PRINTVS000,3060

4) $v(3005+4350)=0(5+430)+25000$

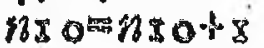

$\rightarrow 48 n \leq 0 \leq 2 n 53$

$n \times 0=0$

a) $v(20 x+n x 0)=006 v(300 x+n s 0)$

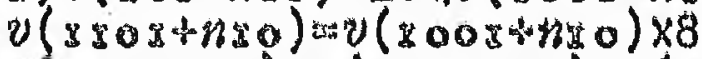

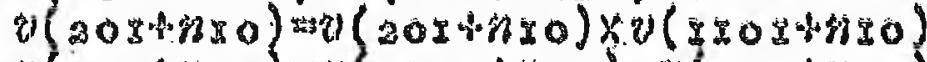

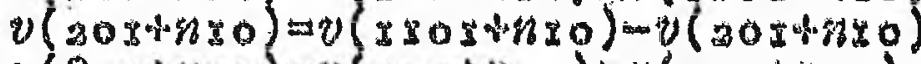

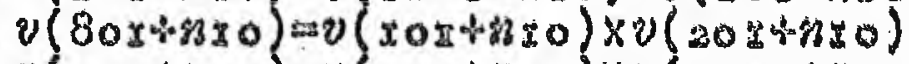

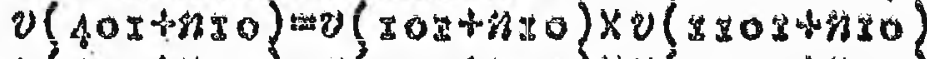

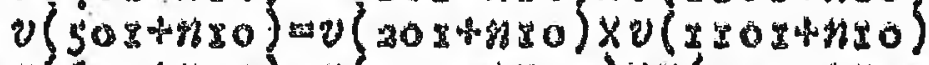

$v\left(60 x+13 x_{0}\right)=v(130 x+11 \times 0) \times v(120 x+n+0)$

$v($ yosrsbso $=v(20 x+450) \times v(30 x+n 30)$

nxosyots

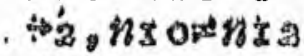

$v_{3} x_{3}=0$

v $3232=0$

$v_{33} 3=0$

$v_{3} 24=0$

v3 $26=0$

$460=0$

3) $v_{3} 3 x=v_{3} 2 x+v(80 x+150)$

$v_{3} s_{3}=v_{3} a x+v(40 x+420)$

$v_{3} 23=v 33 y^{+} v(70 x+840)$

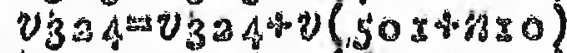

ข325 $=2326+2(608+450)$

nx $0=5 x$

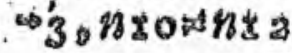




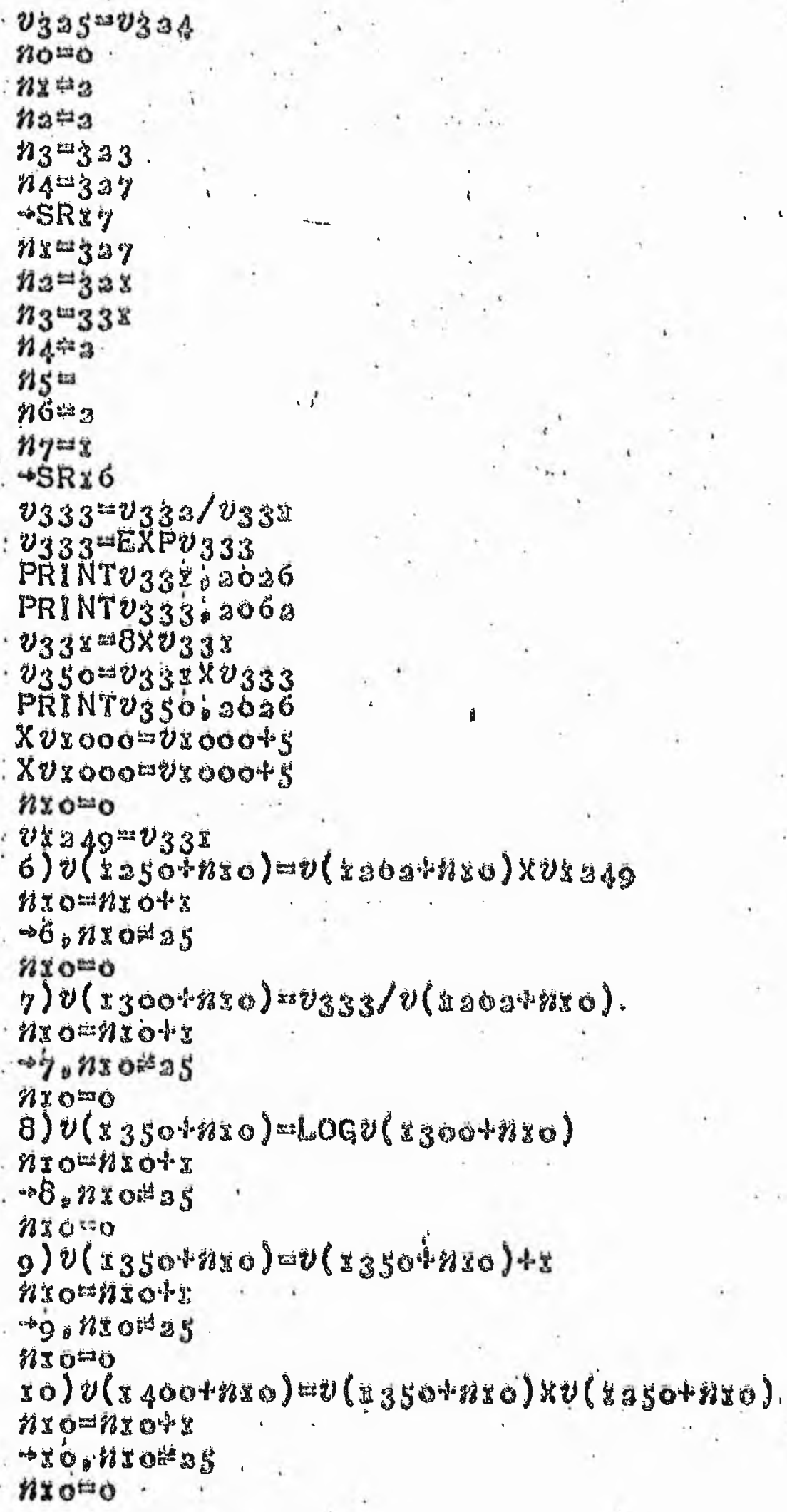


TEXT

T : aX SQD

I. ) PRINTV(

PRINTU $(\$ 400+830) .4024$

$n x O=n \pi 0+2$

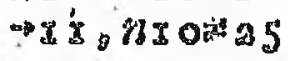

nIo $=0$

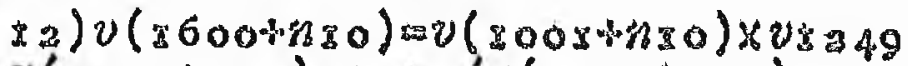

. $v(x 700+510)=833 / v(20054 n 50)$

$v(x 700+4 x \circ)=L, O G v(x 700+n x 0)$

. $v(x 700+1850)=2 x(2700+8720)$

- $v(x 700+780)=v(x 700+8120) \times v(x 600+7 x 0)$

$v(3800+n 50)=v\left(3700 n^{2} n 50\right)-v(30 x+n \leq 0)$

$v(3800+8 \times 0)=v(5800+1120) \times v(x 800+n 20)$

nsomnotr

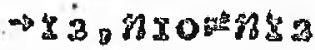

$n 20=0$

VI000:0

I5) $0 \times 900=v 5900+v(8800480)$

$n x \theta=n s 0+8$

$\Rightarrow 55.83004829$

nI $O=0$

ขI950

$V 1900=V \Sigma 900 / V I 950$

$v \Sigma 900=\$ Q R T V I 900$

TEX Ti

DEYIATIOY OF EXPTLO FROM THEORETICAL CURVE

$5=$

PRINTUะ900. 098

$\rightarrow 5$ is Vis00000000

$(\rightarrow 0)$ 


\section{REIPRENCES。}

1. FURZER, I.A., Ph.D.Thesis, University of London, I96I.

2. THOMAS, W.J., and FURZER, I.A., Chem. Eng. Sc., IZ, II5, 1962 。

3. IBRAHIM, $\mathrm{S}_{\circ} \mathrm{H}_{\text {, }}$, and IKULOOR, N.R., Chem, Eng., Sc., II, 1087, I.962.

4. BRINGDAHL, 0, Acta Chem. Scand., 11, $1017,1957$.

5. JOHNSON,P.A.g and BABB,A.L., Chem.Reviews, 56,387, 1956.

6. NIENOW, A.W., Br.Chem. Eng., 10, 827, 1965.

7. TYRELI, H.J.V., Diffusion and Heat Flow in Iiquids, (Butterworths), 1.961.

8. ITGHTFOOT, E.N., CUSSLER,E.L., A.I.Ch.E. Symposium Series, 6I., No.58, 1.965.

9. KAMAL, M.R, , and KANJAR, I.N, , Ch. Eng. Prog, , 62, 82, 1966.

10. CRANK, J., Diffusion in Iiquids, (O.U.P.).

1. JOST, Wog Diffusion, (Academic Press, N.I.).

12. GATNER,J.L., and METZNER, A.B., A.I.Ch.E.- I.Ch.E.

symposium, $6,74,7965$.

13. TUWINER,S:B., Diffusion and Membrane Technology" (Reinhold).

14. GLisSTONE,, g Textbook of Physical Chemistry.

15. ARNOLD,J:H., J.Amex.Chem,Soci, 52, $3937,2930$.

16. GLASSTONE, S., LAIDLER, K, and PYRING, $H_{*}$, Theory of Rate Processes (McGraw-HIII), 194. 
I7. KINCAID, $y_{*}$ F., EYRING, Ho, and STEARN, A. E. ,

Chom.Reva: 28, 301, 1.94.1.

18. HARNED, H. So, and OWEN, $B_{0} B_{1}$, Physical Chemistry of Electrolytic Solutions (Reinhold), 3rd Edn。

19. ONSAGER, $\mathrm{I}_{0}$, and FUOSS, R. M०, J.Phy s。 Chem, , 36,2689, I932。

20. POWELL, R.E. ROSEVEARE, W. E., andEYRTNG, $h_{0}$,

Ind. Eng. Chem. $33,430,1941$.

21. WILKE, C.R., Chem. Eng.Prog。, $45,218,1949$.

22. GARNER, F. $\mathrm{H}_{*}$, and MARCHANT, P.J.M., T. I. Ch, $\mathrm{I}_{*}$,

32, 397,1961 .

23. CALDWEL.T, C.S., and BABB, A.I., J.Phys.Chem, 60, 5I, I956.

24. MUL,ITN, J.W. g GARS IDE, $J_{0}$ g and UNAHABHOKLFA, P.J.M., $J_{.}$Appi. Chem。 , p. 502,1965.

25. DULIIEN, F。A. L, and SHTMILT, I. Wo, Canadian J.Chem。Enge, p. 242, I96I.

26. GORDON, A.R., J.Chor Bhysis, 5 , 522, 1937.

27. ENGLISH, $\Lambda_{0}$, and DOLE, Mag. J.Amer. Chem. Soco, Z2, 3261, 1950 . 28. GOSTING, L.J., and MORRIS, M.S., J.Amer.Chem. Soc., 71, 1998, 194.9.

29. STOKES, R.H., HALII, J.R., and WISHAW, B. F., J.Amer. Chem。Soc., $25,1556,1953$.

30. GAMBIL.t. W.R., Chen. Eng., June 30,1958, p.113.

31. SHERWOOD, T.I. , \&REID, R.C., Properties of Gases and Iiquids (MaGraw-Hill). 
32. THOVERT, G., ComptoRend., 250, 270, 1910 .

33. HERZOG, Z,Phys.Chem。, 149, 89, 1930。

34. OLSON, R.I., and WALTON, J.S., Ind. Eng. Chem,g

43, $704,1951$.

35. SCHEIBEL, E.G., Ind. Eng. Chem., 46, 2007, 1.954.

36. OTHMER, D.F., and THATKAR, M.S.g Ind. Eng.Chom.g

45, 589, 1953。

37. WIEKE, O.Ro, and Pin CHANG, A.I.Ch, Erg. I, 264.1955.

38\% IBRAHIM S.H., Private Communication, 12/11/1965.

39. IBRAHIM $\mathrm{S}_{0} \mathrm{H}_{0}$, and KULOOR,N.R., J.ChemoEng.Data,

$$
\text { 8. 198, 1963. }
$$

40. KING, $\mathrm{C} . \mathrm{J}$. , HiSUEH, I. , and $\mathrm{MAO}, \mathrm{K}$, J.Chem. Eng, Data,

$$
10,348,1965 \text {. }
$$

41. STOKES, R.H., J.Amex.Chem.Soc., Z2, 763, 1950。

42. HARNED, H. S., and NUTPAIL, R.L., J.Amer. Chem.Soc., $69,736,1947$.

43. MULLER,R.H., UCRI Report No.1.1542, Univ, of California.

44. MULLER,R.H., UCRI Report No.11997, UnIV. of Calitomia.

45. CALDWELI, C.S., HALI, J.R., and BABB, A. I.,

Rev.Sc1.Instr., 20, 816,1957.

46. HARVEY, E.A., and SMITH,W., Chem. Eng. Sc.,

$$
\text { 10, 274, } 1959 .
$$

47. BIDLACK, D.I., and ANDERSON,D.K., J.Phys.Chem., 68, 3790,1964 . 
48. IONGSWOREH, I.G. g J.Amer.Chem.Soc., 69, $2510,1947$.

49. IKEGELES, G., and GOSTING, L.J., J.Amex "Chem.Soc., $69,2516,1947$.

50. SVENNSON, $\mathrm{H}_{0}$, Acta Chem.Scand. $3,1170,1949$.

51. SUENNSON, $\mathrm{H}_{0}$, Acta Chem. Scand, 4, 1329, 1950.

52. SVENNSON,H., Acta Chem.Scand., 5, 1301, 1951.

53. SVENINSON, $\mathrm{H}_{\bullet}$, Opta Acta , $3,164,1956$.

54c LEBIEDEFF,M.A. Revod Optiques, 2, 385, 1930.

55. BRINGDAHL, O, Acta Chem. Scand. $12,684,1958$.

56. BRYNGDAHI, $\mathrm{O}_{2}, \mathrm{~g}$ and IJUNGGREI, $\mathrm{S}, \mathrm{g}$ Acta Chem. Scand., 16, 2162, 1962.

57. INGEISTAM, E., J.Opt.Soc.Amer., 42, 536, $195 \%$. 58. THOMAS,W.J., and NICHOLI,E.MK, Applled Optics, 4. $823,1965$.

59. KODAK Data Sheet FM60.

60. AKEIY,D.F., and GOSTING, I.J., J.Amer.Chem.Soc. 75, 5685,1953 .

61. RILEY,J.F., and IXONS, P.A., J.Amer.Chem.Soc., 12, 261, 1955.

62. SCATCHARD, G., J.Amer.Chem.Soc.g 43, 2406, 1921.

63. DOW CHEMICAL INTERNATIONAL, ALkanolamines Fandbook. 64. SHELI CHEMICAI CO, Technical Bullet in GC/42. 65. IEIBUSH, A.G., and SHNEERSON, E.D., J.AppI.Chem. (USSR), 20, 69, 1947. 


\section{$-278$}

66. TROTMAN-DICKENSON, A.F., J.Chem.Soc., p.I293, 1949.

67. CONDON ${ }_{2} \mathrm{~F}_{*} \mathrm{E}_{0}$, J.Amex. Chem. Soc, 87, 44.81, 1965.

68. CONDON, F.E., Private Communication, 12/12/65.

69. PARTINGTON,J.R., Advanced Physical Chemistry,

(Longmans), Vol.4, p.6.

70. SHELI CHEMICAI CO. (Egham Industrial Chemicalis

Laboratory), Private Communication, 28/10/65.

71. PERRY, R.H., Chemical Engineers Handbook (McGraw-Hil.I), 2nd Edno, pp391-392。

72. MCDONALD, $R_{0} A_{\bullet}$, SHRADER, $S_{0} A_{0}$, and STULI, $D_{\bullet} R_{0}$,

$$
\text { J.Chem. Eng, Data., 4, 31.2, } 1959 .
$$

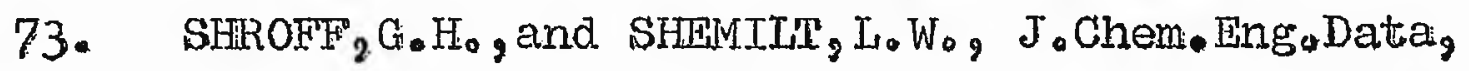

$$
\text { 17, } 183,1966 \text {. }
$$

74. BRYNGDAHH, 0., Ark1V FYstk, 21, 289, 1962.

75. GUSTAFSSON, S. E., BECSEY, J.G, and BIERLEIN, J. A.,

$$
\text { J.Phys.Chema, 69, 1.016, 1965. }
$$

76. CRANK, J., and ROBINSON, $\mathrm{C}_{\text {, }}$ Proc.Royal Soc.,

$$
\text { 204A, 549, 1951. }
$$

77. SECOR, R.M., A.I.Ch.E., II, 452, 1965.

78. Van OSS, C.J., J.Sci. Instr., 4I, 227, 1964.

79. SATO, K., HOSHINO, S., and MTYAMOTO, K.,

Kagakn Kogaku ( English Edn.), 2, 242,1964.

80. THOMAS, W.J., and ADAMS, M.J., Trans.Far. Soc.,

$$
61,668,1965 .
$$


81. THOMAS g W.J. $_{\text {. }}$ A. I. Ch. E. (In press ).

82. WHIIMAN, W. G., Chem. \& Met. Eng, 22, 1923.

83. HIGBIE, R., T.A.I.Ch॰ E., 3.7, 365, 1935.

84. SHERWOOD, T. K., and PIGFORD, R. I., Absorption and Extraction (McGraw-Hill]), and Edn., p.320.

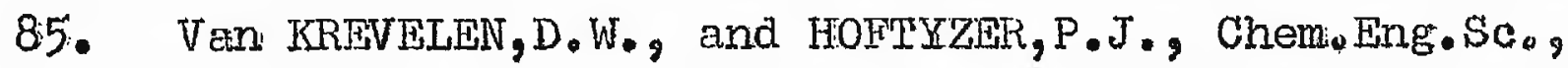
2, $145,1953$.

86. Van KREVELEN, D.W., and HOFTYZER, P.J., T.I.Ch. I., 32, $\mathrm{S} \mathrm{60,} 1954$.

87. BRIAN, P.I.T., Sc.D. Thesis, Univ. of Delaware, 1954。 88. GILLILAND, E.R., BADOUR, R॰F*, and BRIAN, P.I.T., A.I.Ch. E. , $4,223,1958$.

89. BRIAN, P.T.T., HURLEX, J.F., and HASSELITINE, E.H., A. I.Ch.E., Z, 226, 1961.

90. DANKWERTS, P.V., Trans. Far. Soc., 46, 300, 1950.

91. WATSON, H.T., J.Amex.Chem. Soc., 76, 5884, 1954 .

92. CHEMICAL RUBBER PUBLISHING CO., Handbook of Physics and Chemistry, 44th Edn., p3073.

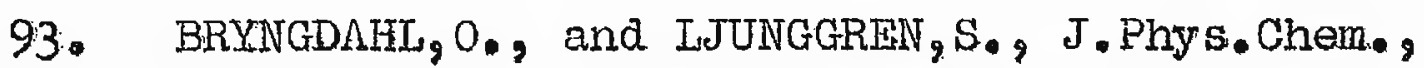
64, 1264, 1960 .

94. BRYNGDAHL, 0., J.opt.Soc.Amer., 53, 571, 1963.

95. HIMMLLBLAU, D.M*, Chem.Reviews, p.527,1964.

96. JEPSEN, J.C., CROSSER, O.K., and PERRY,R.H., A. I.Ch. E., 12, 186, 1966.

97. SHERWOOD, I.K. , and WRI, J.C., A.I.Ch. $E_{0}, 1,522,1955$. 98. MGLLOR, J.W., Higher Mathematics (Dover Publ.) g.286. 
$-280-$

\section{PUBLISHED WORK.}

Reprint from "Applied OptIcs", 4, 823 , July 1965. 


\title{
The Application of the Wavefront Shearing Optical Interferometer to Diffusion Measurements
}

\author{
W. J. Thomas and E. McK. Nicholl
}

\begin{abstract}
The measurement of diffusion coefficients in dilute solutions of electrolytes can be effectively and accurately carried out using birefringence. An interferometer utilizing birefringence has been constructed by the authors and is described. A flowing-junction cell is also used. The four available methods for the calculation of the diffusion coefficient are discussed. A Ferranti Sirius computer has been programmed to handle the calculations. Diffusion coefficients for the systems sucrose-water, monoethanolamine-water, and diethanolamine-water are reported.
\end{abstract}

\section{Introduction}

The determination of diffusion coefficients by an examination of concentration distributions and concentration gradients across a liquid-liquid interface by optical methods is well known. Among the most useful methods are those due to Lamm, Rayleigh, and Gouy. ${ }^{1-3} \quad$ The latter has recently been examined in this laboratory by Thomas and Furzer., 4,5 The MachZender interferometer falls into the same group of optical methods and is also well known.

All the above methods for determining diffusion coefficients rely on the formation of interference fringes. Other optical methods exist, viz., the schlieren scanning and Svennson's diagonal (the slit method), but methods using interference fringes are the most sensitive. Since a shift of one fringe corresponds to a change in refractive index of $5.461 \times 10^{-5}$ for a $1-\mathrm{cm}$ cell $(=$ $\lambda / a$ for the mercury green line), it follows that it is possible to detect extremely small changes in refractive index.

However, a recent development by Ingelstam and Bryngdah ${ }^{6-9}$ is an interferometer with an appreciably greater sensitivity to changes in refractive index than any of the above methods. Bryngdahl has shown that concentrations of as low as $0.01 \%$ can be used. It appears, therefore, that this is the only optical method which can be applied to very dilute electrolyte solutious. It has also been shown that this particular method is capable of giving greater accuracy than previous methods, due to the decrease in the concentration range.

The principal features of the method described by Ingelstam and Bryngdahl are the use of polarized

The authors are with the Department of Chemical Engineering, Battersea College of Technology, London, England.

Received 29 October 1964. light and a Savart plate to form interference fringes. The Savart plate consists of two uniaxial quartz crystals cemented together, with their optic axes at $90^{\circ}$ to each other, at $90^{\circ}$ to the beam direction, and at $45^{\circ}$ with respect to the plane of the paper (see Fig. 1).

Parallel light passes through the first polarizing plate and is polarized such that the oscillation plane bisects the two principal planes of the Savart plate. Thus, the wavefront is divided into two equally strong coherent wavefronts polarized perpendicularly to each other. By means of the second polarizer set at $45^{\circ}$ in relation to the principal planes of the crystal plates, the two wavefronts are caused to interfere.

A complete description of the mechanics of the interference is given by Bryngdahl. ${ }^{7}$

The interference pattern itself consists of a set of horizontal fringe pairs (Fig. 2).

\section{Description}

\section{The Optical System}

The complete optical system, as shown in Fig. 3 is contained in a constant-temperature (thermostatically controlled) darkroom. The temperature of the room can be maintained to $\pm 0.1^{\circ} \mathrm{C}$.

The optical bench is of steel, of triangular section, 3 $\mathrm{m}$ long, resking on two $10-\mathrm{ft}(3.05-\mathrm{m})$ long steel girders bolted to three concrete pillars. (The whole assembly, weighing over $450 \mathrm{~kg}$, is separated from the floor by rubber blockss.) The various components in the optical system are attached to the bench by adjustable saddle stands, fitted with clamping screws.

A 125-W mercury vapor lamp operated in a horizontal position provides a source of monochromatic light (at $5461 \AA$ ). The narrow discharge from the lamp is focused through a shor't focal leng th condensing lens, $L_{1}$, through infrared and green filters onto a horizontal adjustable slit operated from $5 \mu$ to $10 \mu$. 


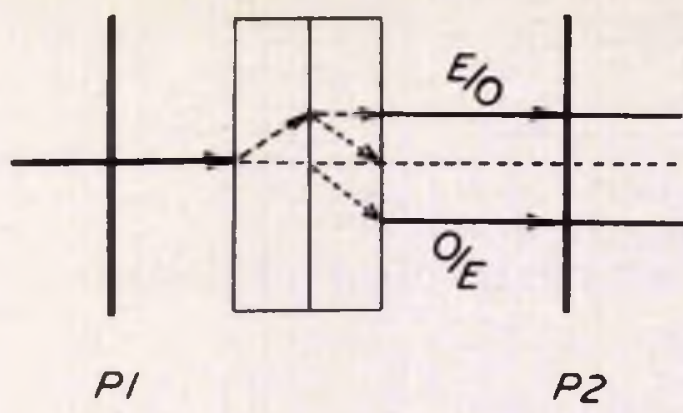

Fig. I. The Savart plate, showing how the incident light ray is split up into an extraordinary/ordinary $(\mathrm{E} / \mathrm{O})$ and an ordinary/ extraordinary $(\mathrm{O} / \mathrm{E})$ ray. $\mathrm{E} / \mathrm{O}$ and $\mathrm{O} / \mathrm{E}$ are perpendicularly polarized to each other.

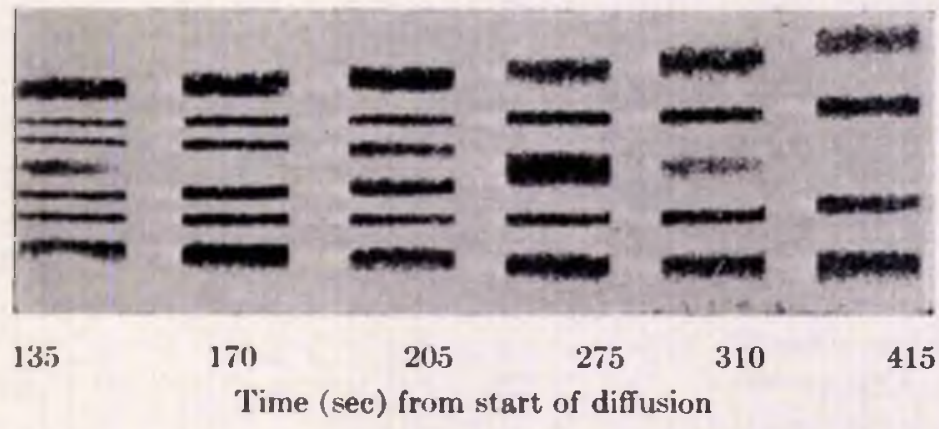

Fig. 2. Photographs of interference fringes recorded during diffusion of $0.0578 \%$ monoethanolamine into water.

To reduce the effect of stray light, the lamp, condenser, filters, and slit are housed in a light-proof structure (which is adequately ventilated).

By means of the similar lenses $L_{2}, L_{3}$, the light is collimated through the flowing-junction cell and also by lens $\mathrm{L}_{4}$. Parallel light is, thus, passed through the Savart plate.

Polarizers $P_{1}$ and $P_{2}$ are placed before $L_{4}$ and after the Savart plate.

The image formed after the Savart plate is rather small, and it is convenient to magnify the final image by means of the lens $\mathrm{L}_{5}$.

The diffusion occurring in the cell is recorded photographically by a $35-\mathrm{mm}$ Robot Recorder camera placed in the image plane, M. The lens is removed from the camera so that the image is projected directly onto the film. The camera is coupled to an automatic time-control unit, by means of which the exposure time and time intervals between exposures may be accurately set.

\section{The Flowing-Junction Cell}

The diffusion cell is of the flowing-junction type and was constructed in the laboratory workshops.

Figure 4 shows the cell with solution reservoirs.

The cell is constructed by bolting together several pieces of ground stainless steel, and grinding the ends plane and parallel. The flowing-junction slit is set at $150 \mu$. Optical glass plates are pressed into contact with the ends of the cell by ten pressure screws. A polythene gasket smeared with silicone grease separates the glass cell ends from the steel flats, while a copper gasket separates the pressure screws from the glass. The optical path length through the cell is $630 \mathrm{~mm}$.

The solution and solvent reservoirs are connected to the cell by a manifold with suitable taps. A mercury reservoir, of variable height, provides the head necessary for the solution and solvent to flow into the cell.

\section{Experimental Technique}

The cell is cleaned and flushed with distilled water and air, before finally being flushed with solvent. The liquid reservoirs are cleaned, flushed, and filled with solvent and solution.

The thermostatic control for the room is set at $25^{\circ} \mathrm{C}$, and the cell and reservoirs are left, set up on the optical bench, for about $12 \mathrm{~h}$ (i.e., overnight). It is important that thermal equilibrium is established when a run is started, for it must be appreciated that small differences in temperature or slight mechanical vibrations transmitted to the cell can cause much more rapid mixing of the diffusing substances than the pure diffusion process itself.

When this is done, the solvent is admitted to the top of the cell and the solution is admitted to the bottom, using the mercury reservoir for the necessary head. The sidestream is then started, and the denser solution slowly rises in the cell chamber so that eventually a sharp interface is formed between solvent and solution at the slit exit. The rate of discharge of the sidestream is critical in establishing an interface, varying between twenty and forty drops per minute depending on the solution used.

Once established, the interface is maintained for about $15 \mathrm{~min}$ in order to be certain that it is quite stable.

Meanwhile the Robot Recorder camera is loaded with a length of Kodak R.55 recording film. A ground glass screen with a magnifying viewer placed in the image plane (for ready inspection of the interface and, later on, the fringes), is removed and the camera is substituted in its place. The automatic time-control unit is set for 5-sec exposures at 30-sec intervals and is switched on. As soon as the first exposure is taken (of the interface), the relevant taps on the cell are shut, thus stopping the sidestream discharge and starting the diffusion process in the cell.

Photographic recording of the diffusion process is continued until inspection of the image reveals that the

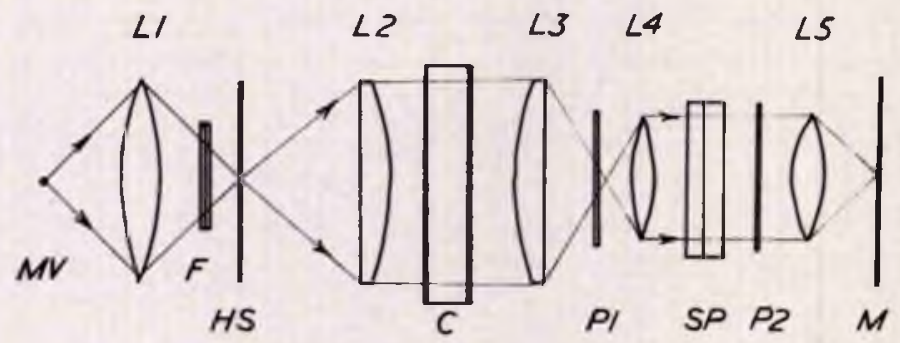

Fig. 3. The wavefront shearing optical interferometer. MV, mercury vapor lamp; F, green and ultraviolet filters; $\mathrm{HS}$, Hilger slit; C, cell; $\mathrm{P}$, polarizers; and $\mathrm{L}$, lenses. 


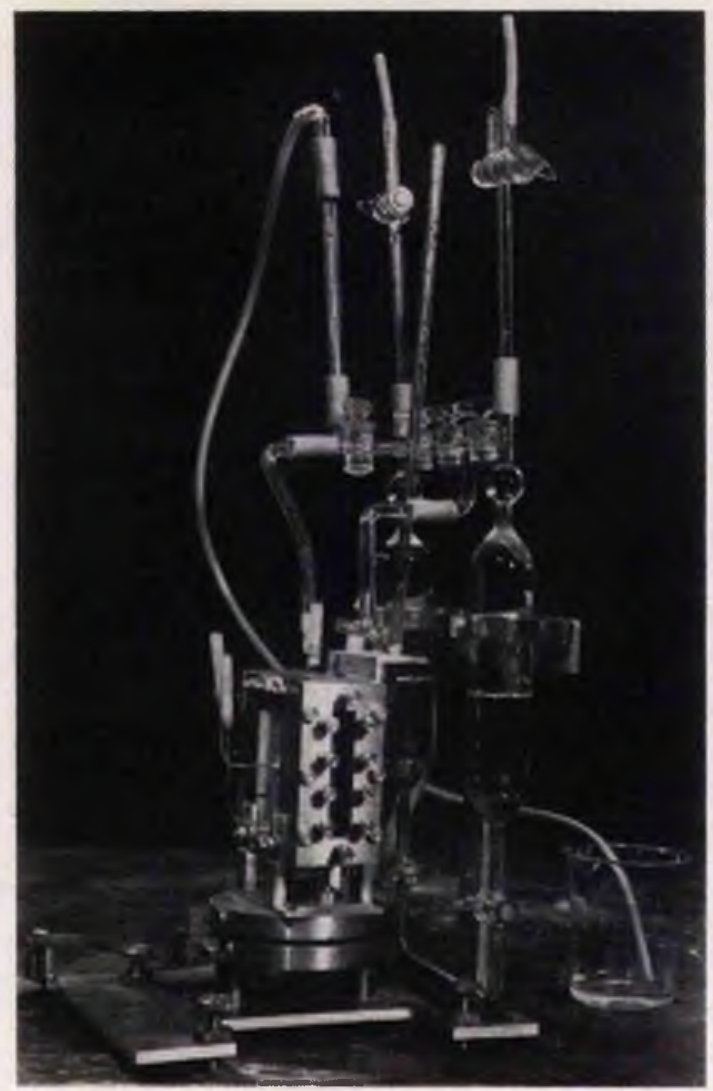

Fig. 4. The flowing-junction cell.

next-to-outermost fringe pair has merged. Depending on the concentration difference between solvent and solution $(0.6 \%$ is convenient) this could occur after about forty exposures.

The exposed film is developed in Kodak DX.80 at a dilution of $1: 4$.

Several high-speed films and developers were tried before it was decided to use the R.55 film and DX.80 developer. R.5i film is sensitive to ultraviolet, blue, and green light-the maximum sensitivity lying between $5400 \AA$ and $5700 \AA .{ }^{10}$ The use of R.55 film is further enhanced by its insensitivity to red light, thus making film handling very easy.

The movement of the fringe pairs as recorded photographically is measured primarily by a recording microdensitometer. Photograms of the fringe pairs are obtained using this instrument, and the distance between the fringe pair peaks can then be measured. Where the recording microdensitometer is limited in accuracy in differentiating the fringe pairs, a cathetometer is used to measure the distance between respective fringe pairs.

Although the movement of several fringe pairs may be observed during the course of a run, it is convenient to follow only one, namely the next to outermost, in order to calculate the diffusion coefficient.

A typical series of fringe pairs is shown in Fig. 2, and associated photograms in Fig. 5 .

\section{Calculation of the Diffusion Coefficient}

The calculation of the diffusion coefficient is based on the basic diffusion equation for the free diffusion case with the diffusion coefficient independent of concentration, i.e.,

$$
\partial c=\frac{c}{2 \sqrt{\pi D} t} \cdot \exp \left(\frac{-z^{2}}{4 D t}\right)
$$

or,

$$
\frac{\partial n}{\partial z}=\frac{\Delta n}{2 \sqrt{\pi D t}} \cdot \exp \left(\frac{-z^{2}}{4 D t}\right) .
$$

Equation (2) follows from Eq. (1), since there is a linear relationship between $c$ and $n$, where

$c$ = concentration of solute diffusing into solute,

$z=$ distance from original boundary in diffusion cell,

$t=$ time

$n$ = refractive index of liquid,

$\Delta n=$ difference in refractive index between initial concentrations of the upper and lower solution in the cell.

Equations (1) and (2) are symmetrical about the origin and have the same form as the Gaussian error curve. The method used here for the evaluation of $D$ is due to Lamm and Bryngdahl, ${ }^{7}$ and the equations used are derived from Eq. (2).

The interference pattern produced is related to the Gaussian curve which represents the ideal concentration gradient, as above, and $D$ is deduced from the rate at which the fringe pattern changes. Following a definite fringe pair with respect to time, we find then the curve representing the movement of this one fringe pair is given by

$$
(2 x)^{2}=8 D t\left(1+\ln t_{i} / t\right)
$$

where

$$
\begin{aligned}
2 x & =\text { distance between fringe pairs } \\
t_{i}= & \text { time corresponding to the maximum distance between } \\
& \text { fringes. }
\end{aligned}
$$

When $t=t_{\text {, }}$

$$
(2 x)_{i}^{2}=8 D t_{i}
$$

The diffusion coefficient, $D$, is obtained from values of $(2 x)^{2}$ and its corresponding times $t_{1}$ and $t_{2}$ by the expression

$$
D=\frac{(2 x)^{2}\left(1 / t_{1}-1 / t_{2}\right)}{8 \cdot \ln t_{2} / t_{1}}
$$

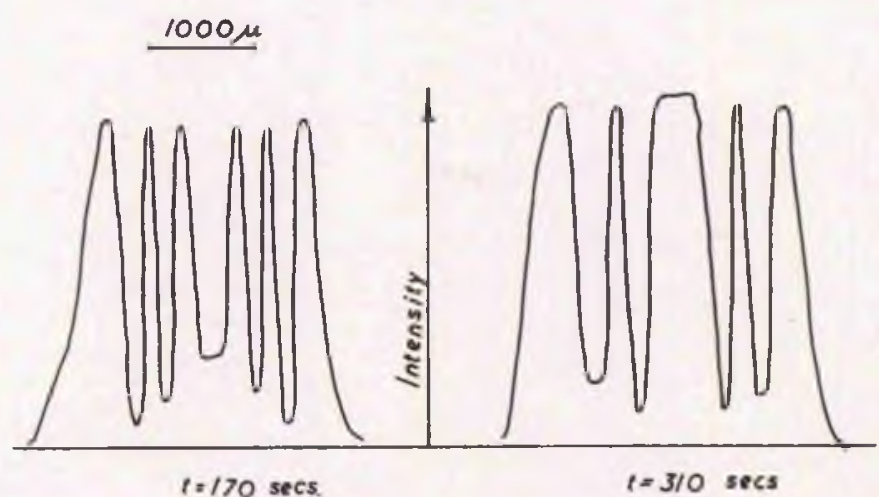

Fig. 5. Actual photograms of interference fringes $(0.0578 \%$ monoethanolamine/water). 


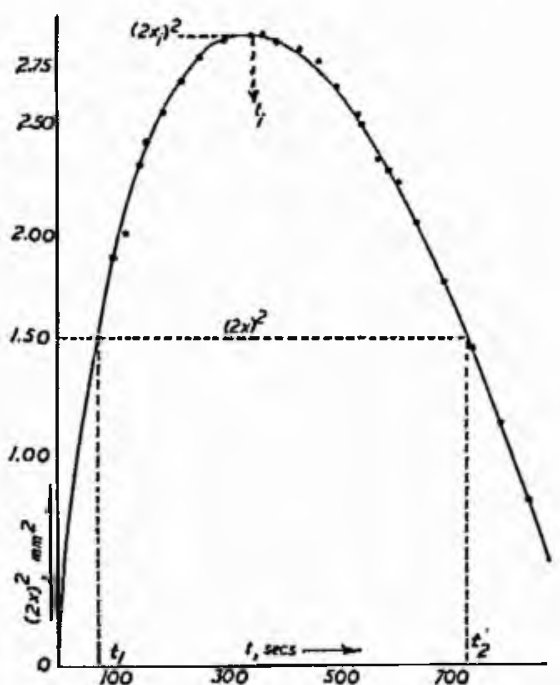

Fig. 6. Griph of $(2 x)^{2}$ against $t,-0.0578 \%$ monoethanolamine-water (next-to-outermost fringe pair). ( $\Delta t=58$ sec added to original $\ell$ values.)

A typical curve of $(2 x)^{2}$ against $t$ is shown in Fig. 6 .

The calculation of $D$, however, is complicated by the fact that the experimental curve obtained is not the true curve.

Due to the experimental impossibility of obtaining an infinitcly sharp interface in the diffusion cell, the experimental curve of $(2 x)^{2}$ against $t$ is, in effect, displaced toward zero along the $t$ axis. It is necessary, therefore, to introduce a zero-time correction factor to Eqs. (3), (4), and (5) in order to calculate the correct value of $D$ [the zero-time factor effectively displacing the curve of $(2 x)^{2}$ against $t$ to its true position, thus enabling the correct value of $D$ to be calculated ]. Thus, the equation of the curve will become:

$$
(2 x)^{2}=8 D(t+\Delta t)\left[1+\ln \left(t_{i}+\Delta t\right) /(t+\Delta t)\right] .
$$

The calculation of $\Delta t$, the zero-time correction factor, may be done by four separate methods as outlined by Bryngdahl. ${ }^{7,11}$ 'Two of the methods are only very limited in accuracy, but the other two methods have proved to be quite acceptable and have been used in the experimental work undertaken in this laboratory. A new technique for calculating $D$ and $\Delta t$ is being perfected by the authors, and this has proved to be considerably more convenient and as accurate as the previous methods. Since a large number of repetitive calculations are involved in the calculation of $D$, use was made of the College's Ferranti Sirius computer. Descriptions of the methods of calculation and associated computer programs are given below.

1. In runs where more than one fringe pair is observed and recorded photographically, these are plotted on a graph of $(2 x)^{2}$ against $t$. A straight line is drawn connecting the maximum points of the curves. The line is of slope $8 D$, and, from the point where it cuts the $l$ axis, $\Delta l$ may be determined. This method is limited in accuracy and has not been used beyond an initial trial.

2. From the basic diffusion equations the logarithmic relationship

$$
(2 x)^{2} / t=C-8 D \ln t_{i} / t
$$

may be derived.

If $(2 x)^{2}$ is plotted against $\ln t$ for any one fringe pair, repeatedly adding a small time-increment to the $t$ values before plotting the curve, then, theoretically, at the stage when the correct $\Delta t$ is reached the curve should be a straight line of slope $-8 D$. In practice, it has been found that the method is very insensitive and is even less accurate than the method outlined above.

3. Bryngdahl has shown that the true $D$ is related to the calculated $D, D^{\prime}$, by the expression

$$
D=D^{\prime} /(1+\Delta t \cdot \nu)
$$

where

$$
\nu=\frac{t_{1}+t_{2}}{t_{1} t_{2}}-\frac{1 / t_{1}-1 / t_{2}}{\ln t_{2} / t_{1}} .
$$

If then $D$ is calculated for any one value of $(2 x)^{2}$ and the corresponding $t_{1}$ and $t_{2}$ values using Eq. (5), and plotted against $\nu$, a straight line is obtained of slope $D \cdot \Delta t$ cutting the ordinate axis at $D$. There are limitations to this method, but it is accurate to the third significant figure. This method has been found most useful as a guide to the accuracy of the experimental points before calculating $D$ using the fourth and most accurate method.

4. The fourth method for calculating $D$ is the most accurate of the four methods outlined here. $D$ is calculated by Eq. (5) and is plotted against $(2 x)^{2}$, with $\Delta t$ as a parameter This is done by first calculating $D$ with no correction applied to the time, for a set of

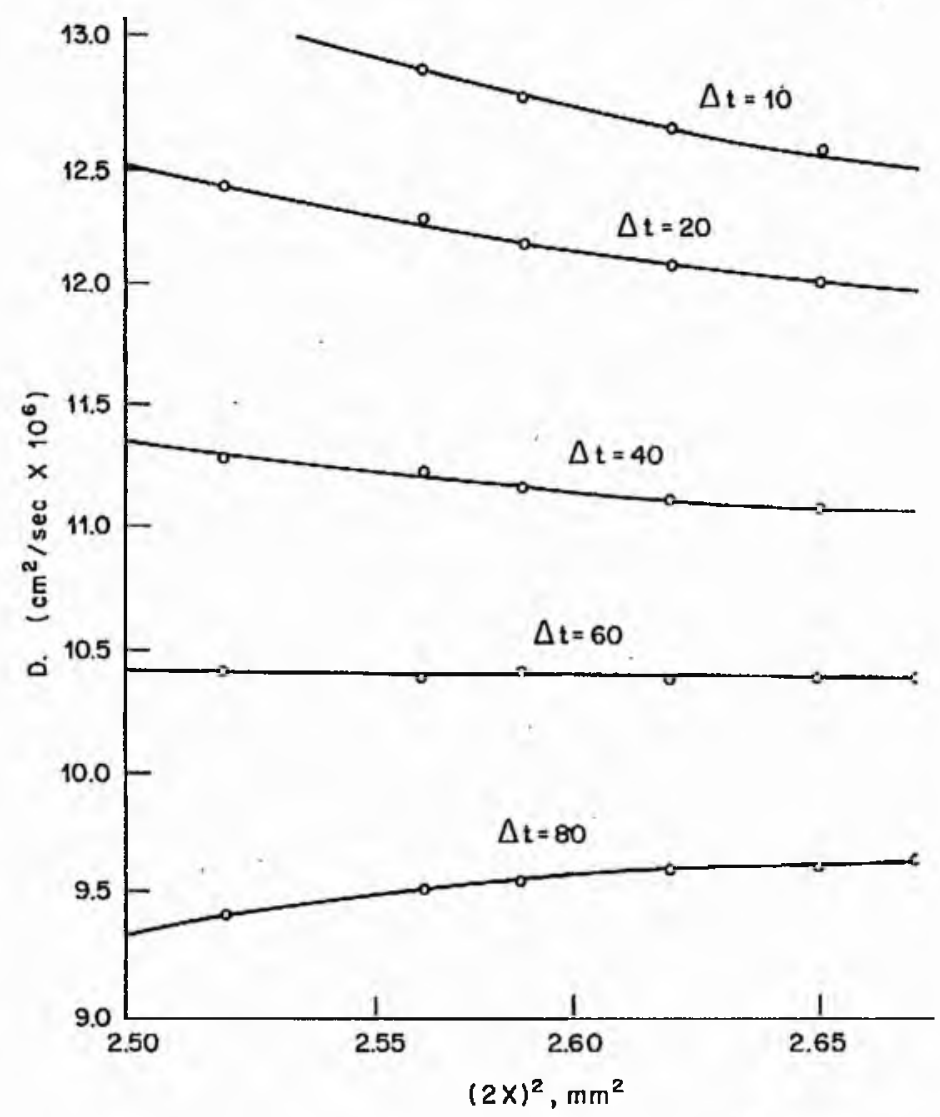

Fig. 7. Graph of $D$ against $(2 x)^{2}, \Delta t$ as parameter $(0.0578 \%)$ monoethanolaminé-water. 
Table I. Diffusion Coefficients Measured Using the Wavefront Shearing Optical Interferometer ${ }^{a}$

Solute

conen.

in dif-

fusing Average

\begin{tabular}{|c|c|c|c|c|c|}
\hline $\begin{array}{l}\text { Run } \\
\text { No. }\end{array}$ & $\begin{array}{l}\text { solution } \\
\text { (wt. \%) }\end{array}$ & $\begin{array}{l}\text { conon. } \\
\text { (wt. \%) }\end{array}$ & $\begin{array}{l}\text { Temp. } \\
\left({ }^{\circ} \mathrm{C}\right)\end{array}$ & $\begin{array}{c}D \\
\left(\mathrm{~cm}^{2} / \mathrm{sec} \times 10^{6}\right)\end{array}$ & $\begin{array}{c}\Delta t \\
(\mathrm{sec})\end{array}$ \\
\hline$s / 1$ & 0.0472 & 0.0236 & 25.5 & $5.221 \pm 0.011$ & 87 \\
\hline $\mathrm{S} / 2$ & 0.0472 & 0.0236 & 25.2 & $5.205 \pm 0.015$ & 22 \\
\hline $\mathbf{S} / 3$ & 0.0667 & 0.0334 & 24.8 & 5.13 & 87 \\
\hline $\mathrm{S} / 4$ & 0.0993 & 0.0497 & 25.3 & $5.216 \pm 0.012$ & 64 \\
\hline $\mathrm{MEA} / \mathbf{1}$ & 0.0578 & 0.0289 & 25.3 & $10.400 \pm 0.100$ & 58 \\
\hline $\mathrm{MEA} / 3$ & $2 \mathrm{~g} \mathrm{mo}$ & l/liter & 25.3 & $8.575 \pm 0.066$ & 22 \\
\hline $\mathrm{MEA} / 4$ & $4 \mathrm{~g} \mathrm{mo}$ & l/liter & 25.3 & $6.729 \pm 0.044$ & 18 \\
\hline $\mathrm{DEA} / 1$ & 0.03259 & 0.0163 & 25.5 & $6.624 \pm 0.040$ & 8 \\
\hline
\end{tabular}

" Runs S/1-4 for sucrose-water, runs MEA/1,3, and 4 for monoethanolamine-water, and run DEA/1 for diethanolaminewater. Magnification factor of the interferometer $=0.563$.

values of $(2 x)^{2}, t_{1}, t_{2}$. Then a small time increment is added to the $t$ values and the calculations are repeated. This is continued until the value of $D$ becomes constant when plotted against $(2 x)^{2}$. At this point the correct value of $D$ is obtained together with the zero-time correction.

The method of calculation is illustrated by Fig. 7 with actual experimental results. It may be seen that $D$ becomes constant at $\Delta t=62 \mathrm{sec}$ and has a value of $10.400 \times 10^{-6} \mathrm{~cm}^{2} / \mathrm{sec}$ at this stiage.

The only drawback to this method is the sheer volume of repetitive calculations if done by hand. To eliminate this a Ferranti Sirius Autocode computer was programmed to handle the calculations.

The program tape is headed off with the calculation method outlined in paragraph 3 above. As stated previously, this calculation serves as a guide to the accuracy of the points calculated by the fourth method.

The flow diagrams for the two computer programs used by the authors are not given here, but would be available on request.

\section{Experimental Results and Discussion}

The interferometer was calibrated using dilute solutions of sucrose-water before further runs were undertaken to investigate the diffusion coefficients of the ethanolamines over a range of concentrations. The sucrose-water results, together with three monoethanolamine- and one diethanolamine-water results are given in Table I. Complete data for the diffusivities of the mono-, di-, and triethanolamines are being accumulated at the moment in these laboratories and will be presented later. Thus the results given in Table I are only a representative sample.

The values of $D$ obtained for sucrose-water agree well with previous experimental values, ${ }^{7}{ }^{12-14}$ obtained by Bryngdahl using a similar interferometer and other workers using the Gouy interferometer; they illustrate the accuracy obtainable and the concentration range in which this interferometer is capable of working.
In run $\mathrm{S} / 3$ it can be seen that an inconsistent value of $D$ was obtained. In this run, thermal equilibrium was not established due to an electrical failure. It was decided to continue with the run, nevertheless, to see what effect this would have on the value of $D$. Since the resulting coefficient is undoubtedly inconsistent, it is most important that thermal equilibrium be established before any run takes place.

The authors have had several opportunities to examine the accuracy obtainable using this interferometer, and to compare the work with that done on the Gouy interferometer in the same laboratory by Furzer. ${ }^{5}$ It is their firm opinion that the wavefront shearing interferometer is superior both in accuracy and convenience of use. The calculation of the results, particularly, is simpler.

The accuracy of the interferometer, naturally, is increased by the quality of the optical components used, and it is essential that the best quality lenses be used in order to utilize the inherent accuracy. There are few problems in the setting up of the interferometer that a basic lmowledge of optics will not resolve, so that it is, in fact, an extremely useful instrument for the type of worls discussed above.

\section{Appendix: a New Method of Calculating the Diffusion Coefficient for Liquid-Liquid Systems}

The design of a wavefront shearing optical interferometer and its use for the measurement of diffusion coefficients in dilute electrolyte solutions has been described in the foregoing sections.

Four methods of calculating the diffusion coefficient were examined, each involving a zero-time correction factor. Such a factor is necessary as the infinitely sharp interface required for the rigorous application of the Fick equation cannot be realized in practice. It was found from direct calculations based on experimental results that of the four methods the iteration method was the most acceptable and accurate. The large volume of repetitive calculations involved in this method was handled by a Terranti Sirius computer.

However, a fault common to all four methods is that the accuracy of the calculations is dependent upon readings taken from a smooth curve drawn through the experimental points on a $(2 x)^{2}$ vs $t$ plot. A slight scatter of points is difficult to avoid experimentally, and this obviously affects the true position of the curve when drawn by hand.

A new method for calculating $D$ is proposed which is less sensitive to error than any of the previously described methods, especially when a slight scatter of points on the experimental curve of $(2 x)^{2}$ vs $t$ cannot be avoided due to limitations of the optical equipment. The new method is also as accurate as the previously mentioned iteration method, and is possibly more convenient to use.

\section{The Method}

The equation for the ideal movement of one fringe pair, as given by Bryngdahl, is used for the calcula- 


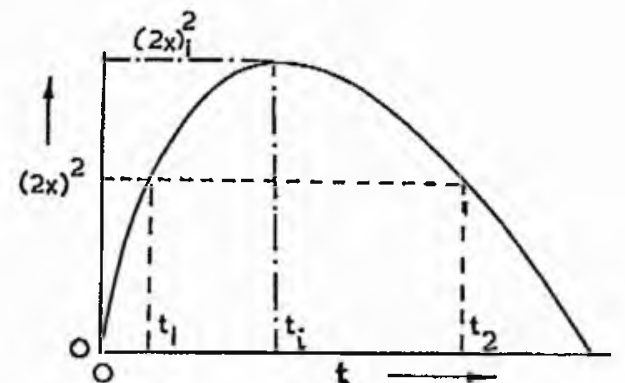

Fig. 8. The ideal curve deseribing the movement of one fringe pair with respect to time.

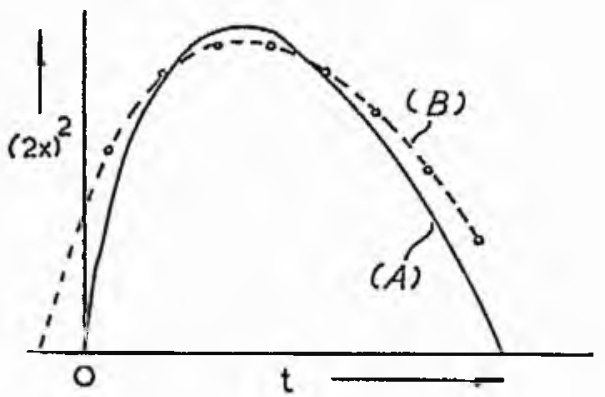

Pig. 9. Ihcorctical curve (A) as obtained from the best $D$ and $t_{i}$ calculated by the method of least squares to fit the experimental points (13). No zero-tide correction applied to B curve.

tions. A fringe pair in the interference pattern (the next to outermost is generally the most convenient to use) is followed with respect to time, and the experimental values of $2 x$ are found. $\quad(2 x)^{2}$ against $t$ is plotted as shown in Fig. 8.

The equation of the curve in Fig. 8 is given by

$$
(2 x)^{2}=8 D t\left[1+\ln \left(t_{i} / t\right)\right]
$$

whore $l_{i}$ is the time corresponding to $(2 x)_{i}{ }^{2}$. When $\iota=l_{i}$,

$$
(2 x)_{i}{ }^{2}=8 D t_{i} .
$$

As mentioned before by the authors, a degree of nonideality is introduced to this equation by the zero-time correction factor, $\Delta t$. Thus the practical equation to be used is

$$
(2 x)^{2}=8 D(t+\Delta t)\left[1+\ln \frac{\left(t_{i}+\Delta t\right)}{(t+\Delta t)}\right] .
$$

Consider now a set of linear equations

$$
\begin{aligned}
y_{1} & =\left(Z_{1} k_{1}\right)_{1}+\left(Z_{2} k_{2}\right)_{1} \\
\text { to } y_{n} & =\left(Z_{1} k_{1}\right)_{n}+\left(Z_{2} k_{2}\right)_{n}
\end{aligned}
$$

in which there are $n$ sets of observations of $y$ and $Z$ values ( $k$ values being constants). It can be shown that the normal equations to determine values for $k_{1}, k_{2}$ that best correlate a set of data $\left(y_{1}, \ldots, y_{n}, x_{1}, \ldots, x_{n}\right)$, where $Z_{1}, Z_{2}=f(x)_{1}, f(x)_{2}$, are:

$$
\begin{aligned}
& k_{1} \sum Z_{1}^{2}+k_{2} \sum Z_{1} Z_{2}=\sum Z_{1} \cdot y, \\
& k_{i} \sum Z_{1} Z_{2}+k_{2} \sum Z_{2}=\sum Z_{2} \cdot y .
\end{aligned}
$$

Values for $k$ are obtained by the simultaneous solution of the normal equations.
Thus for a set of readings of the movement of one fringe pair, the two applicable normal equations to solve for $D$, and $\left(t_{t}+\Delta t\right)$ are:

$$
\begin{aligned}
& D \sum(8 t+\Delta t)^{2}+D \cdot \ln \left(t_{i}+\Delta t\right) \sum(8 t+\Delta t) \\
& \quad \times[(8 t+\Delta t)-(8 t+\Delta t) \cdot \ln (t+\Delta t)]=\sum(2 x)^{2} \cdot D, \\
& D \cdot \sum(8 t+\Delta t)[(8 t+\Delta t)-(8 t+\Delta t) \cdot \ln (t+\Delta t)] \\
& \quad+D \cdot \ln \left(t_{i}+\Delta t\right) \sum[(8 t+\Delta t)-(8 t+\Delta t) \cdot \ln (t+\Delta t)]^{2} \\
& \quad=\sum(2 x)^{2}[(8 t+\Delta t)-(8 t+\Delta t) \cdot \ln (t+\Delta t)]
\end{aligned}
$$

The simultaneous solution of these two equations is best obtained by the use of an algebraic matrix of the form

$$
\left[\begin{array}{l}
\sum Z_{2} \cdot y^{-} \\
\sum Z_{1} \cdot y^{\prime}
\end{array}\right]=\left[\begin{array}{l}
k_{1} \\
k_{2}
\end{array}\right]\left[\begin{array}{ll}
\sum Z_{2}{ }^{2} & \sum Z_{1} Z_{2} \\
\sum Z_{1} Z_{2} & \sum Z_{1}{ }^{2}
\end{array}\right] .
$$

The authors programmed the College's Ferranti Sirius computer to handle the large volume of calculations entailed.

Starting with a value of $\Delta t=0$, the best $D$ and $t_{i}$ are calculated for a set of experimental points. The calculation is repeated for $\Delta t=5,10,15, \ldots$ sec.

It will be found that, when $\Delta t=0$, the curves calculated from the $D$ and $t_{i}$ that best fit the experimental points will be of the same shape as curve $A$ in Fig. 9, i.e., the calculated curve is somewhat more peaked than the curve drawn through the experimental points.

When too large a $\Delta l$ has been applied the calculated curve is less peaked than the experimental curve. Comparing the two values of $(2 x)_{i}{ }^{2}$ i.e., the experimental and the value for the calculated curve, as the calculation proceeds, we find when they coincide that the two curves are identical and the correct value of $D$ and $\Delta t$ may be established.

The authors have used this method of calculation extensively in their work, and the accuracy has proved to be equal to that of the previously mentioned iteration method. However, the method is very much more convenient to use due to its essential simplicity, and is, in fact, quite a rapid method.

Possibly the only limitation to the use of this method is the fact that a fairly complete experimental curve must be obtained which should not be weighted too much to any one side of its peak. This condition, however, would apply just as much to any other method of calculation.

Thanks are due to O. Bryngdahl of The Institute of Optical Research, The Royal Institute of Technology, Stockholm, Sweden, for helpful discussions.

\section{References}

1. H. Tyrell, Diffusion and Heat Flow in Liquids (Butterworths, London, 1961), pp. 75f.

2. L. G. Longsworth, J. Am. Chem. Soc. 69, 2510 (1947).

3. H. Svensson, Acta Chem. Scand. 3, 1170 (1949).

4. W. J. Thomas and I: A. Furzer, Chem. Eng. Sci. 17, 115 (1962). 
5. I. A. Furzer, Ph.D. thesis, University of London (1960).

6. E. Ingelstam, J. Opt. Soc. Am. 47, 536 (1957).

7. O. Bryngdahl, Acta Chem. Scand. 11, 1017 (1957).

8. O. Bryngdahl, Acta Chem. Scand. 12, 684 (1958).

9. O. Bryngdahl, J. Opt. Soc. Am. 53, 571 (1963).

10. Kodnk Data Sheet FM-60.
11. O. Bryngdahl and S. L. Junggren, Acta Chem. Seand. 16, $2162(1962)$.

12. L. J. Gosting and M. S. Morris, J. Am. Chem. Soc. 71, 1998 (1949).

13. D. F. Akely and L. J. Gosting, J. Am. Chem. Soc. 75, 5685 (1953).

14. J. F. Riley and P. A. Lyons, J. Am. Chem. Soc. 77, 261 (1955). 
○ 